ALEA, Lat. Am. J. Probab. Math. Stat. 13, 941-1037 (2016)

DOI: $10.30757 /$ ALEA.v13-36

\title{
Phase diagram for a copolymer in a micro-emulsion
}

\author{
Frank den Hollander and Nicolas Pétrélis
}

Mathematical Institute, Leiden University, P.O. Box 9512, 2300 RA

Leiden, The Netherlands.

E-mail address: denholla@math.leidenuniv.nl

URL: https://www.math.leidenuniv.nl/ denholla/

Laboratoire de Mathématiques Jean Leray UMR 6629, Université de Nantes, 2 Rue de la Houssinière, BP 92208, F-44322 Nantes Cedex 03, France.

E-mail address: nicolas.petrelis@univ-nantes.fr

URL: http: //www.math.sciences.univ-nantes.fr/ petrelis/

\begin{abstract}
In this paper we study a model describing a copolymer in a micro-emulsion. The copolymer consists of a random concatenation of hydrophobic and hydrophilic monomers, the micro-emulsion consists of large blocks of oil and water arranged in a percolation-type fashion. The interaction Hamiltonian assigns energy $-\alpha$ to hydrophobic monomers in oil and energy $-\beta$ to hydrophilic monomers in water, where $\alpha, \beta$ are parameters that without loss of generality are taken to lie in the cone $\left\{(\alpha, \beta) \in \mathbb{R}^{2}: \alpha \geq|\beta|\right\}$. Depending on the values of these parameters, the copolymer either stays close to the oilwater interface (localization) or wanders off into the oil and/or the water (delocalization). Based on an assumption about the strict concavity of the free energy of a copolymer near a linear interface, we derive a variational formula for the quenched free energy per monomer that is column-based, i.e., captures what the copolymer does in columns of different type. We subsequently transform this into a variational formula that is slope-based, i.e., captures what the polymer does as it travels at different slopes, and we use the latter to identify the phase diagram in the $(\alpha, \beta)$-cone. There are two regimes: supercritical (the oil blocks percolate) and subcritical (the oil blocks do not percolate). The supercritical and the subcritical phase diagram each have two localized phases and two delocalized phases, separated by four critical curves meeting at a quadruple critical point. The different phases correspond to the different ways in which the polymer moves through the micro-emulsion. The analysis of the phase diagram is based on three hypotheses about the possible frequencies at which the oil blocks and the water blocks can be visited. We show that these three hypotheses are plausible, but do not provide a proof.
\end{abstract}

Received by the editors June 28, 2013; accepted September 26, 2016.

2010 Mathematics Subject Classification. 60F10, 60K37, 82B27.

Key words and phrases. Random copolymer, random micro-emulsion, free energy, percolation, variational formula, large deviations, concentration of measure.

The research in this paper is supported by ERC Advanced Grant 267356-VARIS. NP is grateful for hospitality at the Mathematical Institute of Leiden University during extended visits in 2011, 2012 and 2013 within the framework of this grant. FdH and NP are grateful for hospitality at the Institute for Mathematical Sciences at the National University of Singapore in May of 2015.

Remark: The part of this paper dealing with the "column-based" variational formula for the free energy has appeared as a preprint on ArXiv: den Hollander and Pétrélis (2012). 


\section{Outline}

In Section 2, we introduce our model for a copolymer in a micro-emulsion and present a variational formula for the quenched free energy per monomer, which we refer to as the slope-based variational formula, involving the fractions of time the copolymer moves at a given slope in the interior of the two solvents and the fraction of time it moves along the interfaces between the two solvents. This variational formula is the corner stone of our analysis. In Section 3, we identify the phase diagram. There are two regimes: supercritical (the oil blocks percolate) and subcritical (the oil blocks do not percolate). We obtain the general structure of the phase diagram, and state a number of properties that exhibit the fine structure of the phase diagram as well. The latter come in the form of theorems and conjectures, and are based on three hypotheses.

In Section 4, we introduce a truncated version of the model in which the copolymer is not allowed to travel more than $M$ blocks upwards or downwards in each column, where $M \in \mathbb{N}$ is arbitrary but fixed. We give a precise definition of the various ingredients that are necessary to state the slope-based variational formula for the truncated model, including various auxiliary quantities that are needed for its proof. Among these is the quenched free energy per monomer of the copolymer crossing a block column of a given type, whose existence and variational characterization are given in Section 5. In Section 6, we derive an auxiliary variational formula for the quenched free energy per monomer in the truncated model, which we refer to as the column-based variational formula, involving both the free energy per monomer and the fraction of time spent inside single columns of a given type. At the end of Section 6, we show how the truncation can be removed by letting $M \rightarrow \infty$. In Section 7, we use the column-based variational formula to derive the slope-based variational formula. In Section 8 we use the slope-based variational formula to prove our results for the phase diagram. Appendices A-G collect several technical results that are needed along the way.

For more background on random polymers with disorder we refer the reader to the monographs by Giacomin (2007) and den Hollander (2009), and to the overview paper by Caravenna et al. (2012).

\section{Model and slope-based variational formula}

In Section 2.1 we define the model, in Section 2.2 we state the slope-based variational formula, in Section 2.3 we place this formula in the proper context.

2.1. Model. To build our model, we distinguish between three scales: (1) the microscopic scale associated with the size of the monomers in the copolymer $(=1$, by convention); (2) the mesoscopic scale associated with the size of the droplets in the micro-emulsion $\left(L_{n} \gg 1\right)$; (3) the macroscopic scale associated with the size of the copolymer $\left(n \gg L_{n}\right)$.

Copolymer configurations. Pick $n \in \mathbb{N}$ and let $\mathcal{W}_{n}$ be the set of $n$-step $d i$ rected self-avoiding paths starting at the origin and being allowed to move upwards, downwards and to the right, i.e.,

$$
\begin{aligned}
& \mathcal{W}_{n}=\left\{\pi=\left(\pi_{i}\right)_{i=0}^{n} \in\left(\mathbb{N}_{0} \times \mathbb{Z}\right)^{n+1}: \pi_{0}=(0,1),\right. \\
& \left.\pi_{i+1}-\pi_{i} \in\{(1,0),(0,1),(0,-1)\} \forall 0 \leq i<n, \pi_{i} \neq \pi_{j} \forall 0 \leq i<j \leq n\right\} .
\end{aligned}
$$


The copolymer is associated with the path $\pi$. The $i$-th monomer is associated with the bond $\left(\pi_{i-1}, \pi_{i}\right)$. The starting point $\pi_{0}$ is chosen to be $(0,1)$ for convenience.

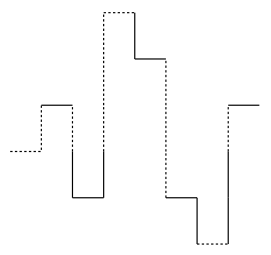

Figure 2.1. Microscopic disorder $\omega$ in the copolymer. Dashed bonds represent monomers of type $A$ (hydrophobic), drawn bonds represent monomers of type $B$ (hydrophilic).

Microscopic disorder in the copolymer. Each monomer is randomly labelled $A$ (hydrophobic) or $B$ (hydrophilic), with probability $\frac{1}{2}$ each, independently for different monomers. The resulting labelling is denoted by

$$
\omega=\left\{\omega_{i}: i \in \mathbb{N}\right\} \in\{A, B\}^{\mathbb{N}}
$$

and represents the randomness of the copolymer, i.e., $\omega_{i}=A$ and $\omega_{i}=B$ mean that the $i$-th monomer is of type $A$, respectively, of type $B$ (see Fig. 2.1). We denote by $\mathbb{P}_{\omega}$ the law of the microscopic disorder.

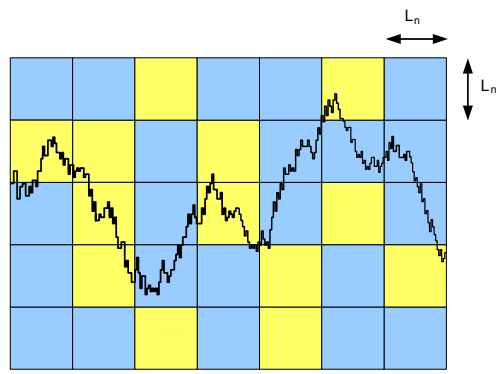

Figure 2.2. Mesoscopic disorder $\Omega$ in the micro-emulsion. Light shaded blocks represent droplets of type $A$ (oil), dark shaded blocks represent droplets of type $B$ (water). Drawn is also the copolymer, but without an indication of the microscopic disorder $\omega$ attached to it.

Mesoscopic disorder in the micro-emulsion. Fix $p \in(0,1)$ and $L_{n} \in \mathbb{N}$. Partition $(0, \infty) \times \mathbb{R}$ into square blocks of size $L_{n}$ :

$$
(0, \infty) \times \mathbb{R}=\bigcup_{x \in \mathbb{N}_{0} \times \mathbb{Z}} \Lambda_{L_{n}}(x), \quad \Lambda_{L_{n}}(x)=x L_{n}+\left(0, L_{n}\right]^{2} .
$$

Each block is randomly labelled $A$ (oil) or $B$ (water), with probability $p$, respectively, $1-p$, independently for different blocks. The resulting labelling is denoted by

$$
\Omega=\left\{\Omega(x): x \in \mathbb{N}_{0} \times \mathbb{Z}\right\} \in\{A, B\}^{\mathbb{N}_{0} \times \mathbb{Z}}
$$


and represents the randomness of the micro-emulsion, i.e., $\Omega(x)=A$ and $\Omega(x)=B$ mean that the $x$-th block is of type $A$, respectively, of type $B$ (see Fig. 2.2). The law of the mesoscopic disorder is denoted by $\mathbb{P}_{\Omega}$ and is independent of $\mathbb{P}_{\omega}$. The size of the blocks $L_{n}$ is assumed to be non-decreasing and to satisfy

$$
\lim _{n \rightarrow \infty} L_{n}=\infty \text { and } \lim _{n \rightarrow \infty} \frac{\log n}{n} L_{n}=0,
$$

i.e., the blocks are large compared to the monomer size but small compared to the copolymer size. For convenience we assume that if an $A$-block and a $B$-block are next to each other, then the interface belongs to the $A$-block.

Hamiltonian and free energy. Given $\omega, \Omega$ and $n$, with each path $\pi \in \mathcal{W}_{n}$ we associate an energy given by the Hamiltonian

$$
H_{n, L_{n}}^{\omega, \Omega}(\pi ; \alpha, \beta)=\sum_{i=1}^{n}\left(\alpha 1\left\{\omega_{i}=\Omega_{\left(\pi_{i-1}, \pi_{i}\right)}^{L_{n}}=A\right\}+\beta 1\left\{\omega_{i}=\Omega_{\left(\pi_{i-1}, \pi_{i}\right)}^{L_{n}}=B\right\}\right)
$$

where $\Omega_{\left(\pi_{i-1}, \pi_{i}\right)}^{L_{n}}$ denotes the label of the block the step $\left(\pi_{i-1}, \pi_{i}\right)$ lies in. What this Hamiltonian does is count the number of $A A$-matches and $B B$-matches and assign them energy $\alpha$ and $\beta$, respectively, where $\alpha, \beta \in \mathbb{R}$. (Note that the interaction is assigned to bonds rather than to sites, and that we do not follow the convention of putting a minus sign in front of the Hamiltonian.) Similarly to what was done in our earlier papers den Hollander and Whittington (2006), den Hollander and Pétrélis (2009a,b, 2010), without loss of generality we may restrict the interaction parameters to the cone

$$
\mathrm{CONE}=\left\{(\alpha, \beta) \in \mathbb{R}^{2}: \alpha \geq|\beta|\right\} .
$$

For $n \in \mathbb{N}$, the free energy per monomer is defined as

$$
f_{n}^{\omega, \Omega}(\alpha, \beta)=\frac{1}{n} \log Z_{n, L_{n}}^{\omega, \Omega}(\alpha, \beta) \quad \text { with } \quad Z_{n, L_{n}}^{\omega, \Omega}(\alpha, \beta)=\sum_{\pi \in \mathcal{W}_{n}} e^{H_{n, L_{n}}^{\omega, \Omega}(\pi ; \alpha, \beta)},
$$

and in the limit as $n \rightarrow \infty$ the free energy per monomer is given by

$$
f(\alpha, \beta ; p)=\lim _{n \rightarrow \infty} f_{n, L_{n}}^{\omega, \Omega}(\alpha, \beta),
$$

provided this limit exists $\omega, \Omega$-a.s.

Henceforth, we subtract the term $\alpha \sum_{i=1}^{n} 1\left\{\omega_{i}=A\right\}$ from the Hamiltonian, which by the law of large numbers $\omega$-a.s. is $\frac{\alpha}{2} n(1+o(1))$ as $n \rightarrow \infty$ and corresponds to a shift of $-\frac{\alpha}{2}$ in the free energy. The latter transformation allows us to lighten the notation, starting with the Hamiltonian in (2.6), which becomes

$$
H_{n, L_{n}}^{\omega, \Omega}(\pi ; \alpha, \beta)=\sum_{i=1}^{n}\left(\beta 1\left\{\omega_{i}=B\right\}-\alpha 1\left\{\omega_{i}=A\right\}\right) 1\left\{\Omega_{\left(\pi_{i-1}, \pi_{i}\right)}^{L_{n}}=B\right\} .
$$

2.2. The slope-based variational formula for the quenched free energy per step. Theorem 2.1 below gives a variational formula for the free energy per step in (2.9). This variational formula, which is the corner stone of our paper, involves the fractions of time the copolymer moves at a given slope through the interior of solvents $A$ and $B$ and the fraction of time it moves along $A B$-interfaces. This variational formula will be crucial to identify the phase diagram, i.e., to identify the typical behavior of the copolymer in the micro-emulsion as a function of the parameters $\alpha, \beta, p$ (see Section 3 for theorems and conjectures). Of particular interest is the distinction 
between localized phases, where the copolymer stays close to the $A B$-interfaces, and delocalized phases, where it wanders off into the solvents $A$ and/or $B$. We will see that there are several such phases.

To state Theorem 2.1 we need to introduce some further notation. With each $l \in \mathbb{R}_{+}=[0, \infty)$ we associate two numbers $v_{A, l}, v_{B, l} \in[1+l, \infty)$ indicating how many steps per horizontal step the copolymer takes when travelling at slope $l$ in solvents $A$ and $B$, respectively. We further let $v_{\mathcal{I}} \in[1, \infty)$ denote the number of steps per horizontal step the copolymer takes when travelling along $A B$-interfaces. These numbers are gathered into the set

$$
\overline{\mathcal{B}}=\left\{v=\left(v_{A}, v_{B}, v_{\mathcal{I}}\right) \in \mathcal{C} \times \mathcal{C} \times[1, \infty)\right\}
$$

with

$$
\mathcal{C}=\left\{l \mapsto u_{l} \text { on } \mathbb{R}_{+}: \text {continuous with } u_{l} \geq 1+l \forall l \in \mathbb{R}_{+}\right\} .
$$

Let $\tilde{\kappa}(u, l)$ be the entropy per step carried by trajectories moving at slope $l$ with the constraint that the total number of steps divided by the total number of horizontal steps is equal to $u \in[1+l, \infty$ ) (for more details, see Section 4.1). Let $\phi_{\mathcal{I}}(u ; \alpha, \beta)$ be the free energy per step when the copolymer moves along an $A B$ interface, with the constraint that the total number of steps divided by the total number of horizontal steps is equal to $u \in[1, \infty)$ (for more details, see Section 4.2). Let $\bar{\rho}=\left(\rho_{A}, \rho_{B}, \rho_{\mathcal{I}}\right) \in \mathcal{M}_{1}\left(\mathbb{R}_{+} \times \mathbb{R}_{+} \times\{\mathcal{I}\}\right)$, where $\bar{\rho}_{A}(d l)$ and $\bar{\rho}_{B}(d l)$ denote the fractions of horizontal steps at which the copolymer travels through solvents $A$ and $B$ at a slope that lies between $l$ and $l+d l$, and $\rho_{\mathcal{I}}$ denotes the fraction of horizontal steps at which the copolymer travels along $A B$-interfaces. The possible values of $\bar{\rho}$ form a set

$$
\overline{\mathcal{R}}_{p} \subset \mathcal{M}_{1}\left(\mathbb{R}_{+} \times \mathbb{R}_{+} \times\{\mathcal{I}\}\right)
$$

that depends on $p$ (for more details, see Section 4.5). With these ingredients we can now state our slope-based variational formula.

Theorem 2.1. [slope-based variational formula] For every $(\alpha, \beta) \in$ CONE and $p \in(0,1)$ the free energy in $(2.9)$ exists for $\mathbb{P}$-a.e. $(\omega, \Omega)$ and in $L^{1}(\mathbb{P})$, and is given by

$$
f(\alpha, \beta ; p)=\sup _{\bar{\rho} \in \overline{\mathcal{R}}_{p}} \sup _{v \in \overline{\mathcal{B}}} \frac{\bar{N}(\bar{\rho}, v)}{\bar{D}(\bar{\rho}, v)}
$$

where

$$
\begin{aligned}
\bar{N}(\bar{\rho}, v)= & \int_{0}^{\infty} v_{A, l} \tilde{\kappa}\left(v_{A, l}, l\right) \bar{\rho}_{A}(d l)+\int_{0}^{\infty} v_{B, l}\left[\tilde{\kappa}\left(v_{B, l}, l\right)+\frac{\beta-\alpha}{2}\right] \bar{\rho}_{B}(d l) \\
& +v_{\mathcal{I}} \phi_{\mathcal{I}}\left(v_{\mathcal{I}} ; \alpha, \beta\right) \bar{\rho}_{\mathcal{I}} \\
\bar{D}(\bar{\rho}, v)= & \int_{0}^{\infty} v_{A, l} \bar{\rho}_{A}(d l)+\int_{0}^{\infty} v_{B, l} \bar{\rho}_{B}(d l)+v_{\mathcal{I}} \bar{\rho}_{\mathcal{I}}
\end{aligned}
$$

with the convention that $\bar{N}(\bar{\rho}, v) / \bar{D}(\bar{\rho}, v)=-\infty$ when $\bar{D}(\bar{\rho}, v)=\infty$.

Remark 2.2. In order to obtain (2.15), we need to assume strict concavity of an auxiliary free energy, involving a copolymer in the vicinity of a single linear interface. This is the object of Assumption 4.3 in Section 4.2, which is supported by a brief discussion. 
2.3. Discussion. The variational formula in $(2.14-2.15)$ is tractable, to the extent that the $\tilde{\kappa}$-function is known explicitly, the $\phi_{\mathcal{I}}$-function has been studied in depth in the literature (and much is known about it), while the set $\overline{\mathcal{B}}$ is simple. The key difficulty of (2.14-2.15) resides in the set $\overline{\mathcal{R}}_{p}$, whose structure is not easy to control. A detailed study of this set is not within the scope of our paper. Fortunately, it turns out that we need to know relatively little about $\overline{\mathcal{R}}_{p}$ in order to identify the general structure of the phase diagram (see Section 3). With the help of three hypotheses on $\overline{\mathcal{R}}_{p}$, each of which is plausible, we can also identify the fine structure of the phase diagram (see Section 3.2).

We expect that the supremum in (2.14) is attained at a unique $\bar{\rho} \in \overline{\mathcal{R}}_{p}$ and a unique $v \in \overline{\mathcal{B}}$. This maximizer corresponds to the copolymer having a specific way to configure itself optimally within the micro-emulsion.

Column-based variational formula. The slope-based variational formula in Theorem 2.1 will be obtained by combining two auxiliary variational formulas. Both formulas involve the free energy per step $\psi\left(\Theta, u_{\Theta} ; \alpha, \beta\right)$ when the copolymer crosses a block column of a given type $\Theta$, taking values in a type space $\overline{\mathcal{V}}$, for a given $u_{\Theta} \in \mathbb{R}^{+}$that indicates how many steps on scale $L_{n}$ the copolymer makes in this column type. A precise definition of this free energy per block column will be given in Section 4.4.2.

The first auxiliary variational formula is stated in Section 4 (Proposition 4.6) and gives an expression for $\psi\left(\Theta, u_{\Theta} ; \alpha, \beta\right)$ that involves the entropy $\tilde{\kappa}(\cdot, l)$ of the copolymer moving at a given slope $l$ and the quenched free energy per monomer $\phi_{\mathcal{I}}$ of the copolymer near a single linear interface. Consequently, the free energy of our model with a random geometry is directly linked to the free energy of a model with a non-random geometry. This will be crucial for our analysis of the phase diagram in Section 3. The microscopic disorder manifests itself only through the free energy of the linear interface model.

The second auxiliary variational formula is stated in Section 6 (Proposition 6.1). It is referred to as the column-based variational formula, and provides an expression for $f(\alpha, \beta ; p)$ by using the block-column free energies $\psi\left(\Theta, u_{\Theta} ; \alpha, \beta\right)$ for $\Theta \in \overline{\mathcal{V}}$ and by weighting each column type with the frequency $\rho(d \Theta)$ at which it is visited by the copolymer. The numerator is the total free energy, the denominator is the total number of monomers (both on the mesoscopic scale). The variational formula contains suprema over $\left(u_{\Theta}\right)_{\Theta \in \overline{\mathcal{V}}} \in \mathcal{B}_{\overline{\mathcal{V}}}$ and $\rho \in \mathcal{R}_{p}$. The reason why these two suprema appear in (6.2) is that, as a consequence of assumption (2.5), the mesoscopic scale carries no entropy: all the entropy comes from the microscopic scale, through the free energy per monomer in single columns. The mesoscopic disorder manifests itself only through the presence of the set $\mathcal{R}_{p}$.

Removal of the corner restriction. In our earlier papers den Hollander and Whittington (2006), den Hollander and Pétrélis (2009a,b, 2010), we allowed the configurations of the copolymer to be given by the subset of $\mathcal{W}_{n}$ consisting of those paths that enter pairs of blocks through a common corner, exit them at one of the two corners diagonally opposite and in between stay confined to the two blocks that are seen upon entering. The latter is an unphysical restriction that was adopted to simplify the model. In these papers we derived a variational formula for the free energy per step that had a much simpler structure. We analyzed this variational formula as a function of $\alpha, \beta, p$ and found that there are two regimes, supercritical and subcritical, depending on whether the oil blocks percolate or not along the 
coarse-grained self-avoiding path running along the corners. In the supercritical regime the phase diagram turned out to have two phases, in the subcritical regime it turned out to have four phases, meeting at two tricritical points.

In Section 3 we show how the variational formula in Theorem 2.1 can be used to identify the phase diagram. It turns out that there are two types of phases: localized phases (where the copolymer spends a positive fraction of its time near the $A B$-interfaces) and delocalized phases (where it spends a zero fraction of its time near the $A B$-interfaces). Which of these phases occurs depends on the parameters $\alpha, \beta, p$. It is energetically favorable for the copolymer to stay close to the $A B$ interfaces, where it has the possibility of placing more than half of its monomers in their preferred solvent (by switching sides when necessary), but this comes with a loss of entropy. The competition between energy and entropy is controlled by the energy parameters $\alpha, \beta$ (determining the reward of switching sides) and by the density parameter $p$ (determining the density of the $A B$-interfaces). It turns out that the phase diagram is different in the supercritical and the subcritical regimes, where the $A$-blocks percolate, respectively, do not percolate. The phase diagram is richer than for the model with the corner restriction.

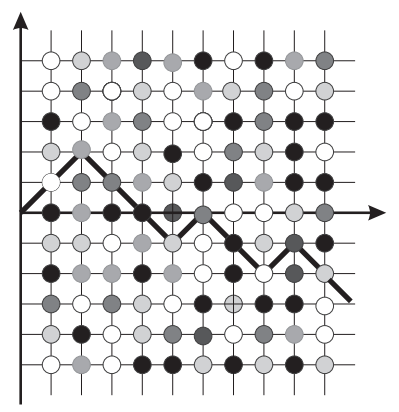

FiguRE 2.3. Picture of a directed polymer with bulk disorder. The different shades of black, grey and white represent different values of the disorder.

Comparison with the directed polymer with bulk disorder. A model of a polymer with disorder that has been studied intensively in the literature is the directed polymer with bulk disorder. Here, the set of paths is

$$
\mathcal{W}_{n}=\left\{\pi=\left(i, \pi_{i}\right)_{i=0}^{n} \in\left(\mathbb{N}_{0} \times \mathbb{Z}^{d}\right)^{n+1}: \pi_{0}=0,\left\|\pi_{i+1}-\pi_{i}\right\|=1 \forall 0 \leq i<n\right\},
$$

where $\|\cdot\|$ is the Euclidean norm on $\mathbb{Z}^{d}$, and the Hamiltonian is

$$
H_{n}^{\omega}(\pi)=\lambda \sum_{i=1}^{n} \omega\left(i, \pi_{i}\right),
$$

where $\lambda>0$ is a parameter and $\omega=\left\{\omega(i, x): i \in \mathbb{N}, x \in \mathbb{Z}^{d}\right\}$ is a field of i.i.d. $\mathbb{R}$-valued random variables with zero mean, unit variance and finite moment generating function, where $\mathbb{N}$ is time and $\mathbb{Z}^{d}$ is space (see Fig. 2.3). This model can be viewed as a version of a copolymer in a micro-emulsion where the droplets are of the same size as the monomers. For this model a variational formula for the free energy has been derived by Rassoul-Agha et al. (2013, 2016+). However, the variational formula is abstract and therefore does not lead to a quantitative understanding of 
the phase diagram. Most of the analysis in the literature relies on the application of martingale techniques (for details, see e.g. den Hollander, 2009, Chapter 12).

In our model (which is restricted to $d=1$ and has self-avoiding paths that may move north, south and east instead of north-east and south-east), the droplets are much larger than the monomers. This causes a self-averaging of the microscopic disorder, both when the copolymer moves inside one of the solvents and when it moves near an interface. Moreover, since the copolymer is much larger than the droplets, also self-averaging of the mesoscopic disorder occurs. This is why the free energy can be expressed in terms of a variational formula, as in Theorem 2.1. This variational formula acts as a jumpboard for a detailed analysis of the phase diagram. Such a detailed analysis is lacking for the directed polymer with bulk disorder.

The directed polymer in random environment has two phases: a weak disorder phase (where the quenched and the annealed free energy are asymptotically comparable) and a strong disorder phase (where the quenched free energy is asymptotically smaller than the annealed free energy). The strong disorder phase occurs in dimension $d=1,2$ for all $\lambda>0$ and in dimension $d \geq 3$ for $\lambda>\lambda_{c}$, with $\lambda_{c} \in[0, \infty]$ a critical value that depends on $d$ and on the law of the disorder. It is predicted that in the strong disorder phase the copolymer moves within a narrow corridor that carries sites with high energy (recall our convention of not putting a minus sign in front of the Hamiltonian), resulting in superdiffusive behavior in the spatial direction. We expect a similar behavior to occur in the localized phases of our model, where the polymer targets the $A B$-interfaces. It would be interesting to find out how far the coarsed-grained self-avoiding path in our model travels vertically as a function of $n$.

\section{Phase diagram}

In Section 3.1 we identify the general structure of the phase diagram. In particular, we show that there is a localized phase $\mathcal{L}$ in which $A B$-localization occurs, and a delocalized phase $\mathcal{D}$ in which no $A B$-localization occurs. In Section 3.2, we obtain various results for the fine structure of the phase diagram, both for the supercritical regime $p>p_{c}$ and for the subcritical regime $p<p_{c}$, where $p_{c}$ denotes the critical threshold for directed bond percolation in the positive quandrant of $\mathbb{Z}^{2}$. This fine structure comes in the form of theorems and conjectures, and is based on three hypotheses, which we discuss in Section 3.3.

3.1. General structure. To state the general structure of the phase diagram, we need to define a reduced version of the free energy, called the delocalized free energy $f_{\mathcal{D}}$, obtained by taking into account those trajectories that, when moving along an $A B$-interface, are delocalized in the $A$-solvent. The latter amounts to replacing the linear interface free energy $\phi_{\mathcal{I}}\left(v_{\mathcal{I}} ; \alpha, \beta\right)$ in $(2.14)$ by the entropic constant lower bound $\tilde{\kappa}\left(v_{\mathcal{I}}, 0\right)$. Thus, we define

$$
f_{\mathcal{D}}(\alpha, \beta ; p)=\sup _{\bar{\rho} \in \overline{\mathcal{R}}_{p}} \sup _{v \in \overline{\mathcal{B}}} \frac{\bar{N}_{\mathcal{D}}(\bar{\rho}, v)}{\bar{D}_{\mathcal{D}}(\bar{\rho}, v)}
$$

$$
\begin{aligned}
& \text { with } \\
& \bar{N}_{\mathcal{D}}(\bar{\rho}, v)=\int_{0}^{\infty} v_{A, l} \tilde{\kappa}\left(v_{A, l}, l\right)\left[\bar{\rho}_{A}+\bar{\rho}_{\mathcal{I}} \delta_{0}\right](d l)+\int_{0}^{\infty} v_{B, l}\left[\tilde{\kappa}\left(v_{B, l}, l\right)+\frac{\beta-\alpha}{2}\right] \bar{\rho}_{B}(d l), \\
& \bar{D}_{\mathcal{D}}(\bar{\rho}, v)=\int_{0}^{\infty} v_{A, l}\left[\bar{\rho}_{A}+\bar{\rho}_{\mathcal{I}} \delta_{0}\right](d l)+\int_{0}^{\infty} v_{B, l} \bar{\rho}_{B}(d l)
\end{aligned}
$$


provided $\bar{D}_{\mathcal{D}}(\bar{\rho}, v)<\infty$. Note that $f_{\mathcal{D}}(\alpha, \beta ; p)$ depends on $(\alpha, \beta)$ through $\alpha-\beta$ only.

We partition the CONE into the two phases $\mathcal{D}$ and $\mathcal{L}$ defined by

$$
\begin{aligned}
& \mathcal{L}=\left\{(\alpha, \beta) \in \mathrm{CONE}: f(\alpha, \beta ; p)>f_{\mathcal{D}}(\alpha, \beta ; p)\right\}, \\
& \mathcal{D}=\left\{(\alpha, \beta) \in \mathrm{CONE}: f(\alpha, \beta ; p)=f_{\mathcal{D}}(\alpha, \beta ; p)\right\}
\end{aligned}
$$

The localized phase $\mathcal{L}$ corresponds to large values of $\beta$, for which the energetic reward to spend some time travelling along $A B$-interfaces exceeds the entropic penalty to do so. The delocalized phase $\mathcal{D}$, on the other hand, corresponds to small values of $\beta$, for which the energetic reward does not exceed the entropic penalty.

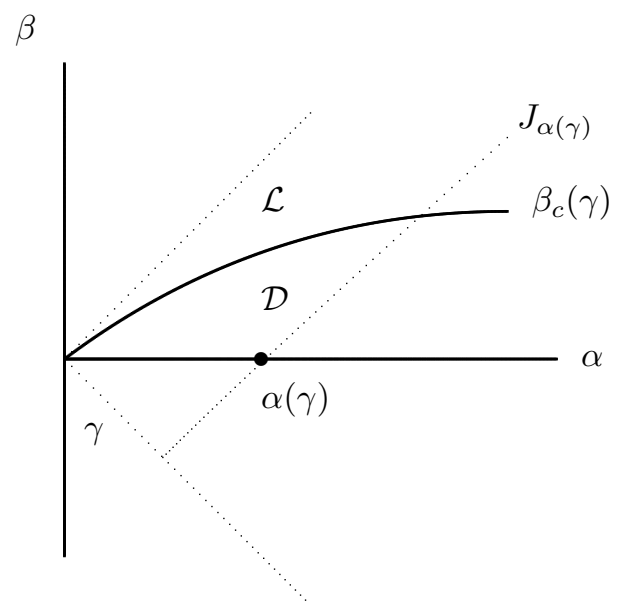

Figure 3.4. Qualitative picture of the phase diagram in CONE. The curve $\gamma \mapsto \beta_{c}(\gamma)$ separates the localized phase $\mathcal{L}$ from the delocalized phase $\mathcal{D}$. The parameter $\gamma$ measures the distance between the origin and the point on the lower boundary of CONE from which the line with slope 1 hits the curve at height $\beta(\gamma)$. Note that $\alpha(\gamma)=\gamma \sqrt{2}$ is the value where this line crosses the horizontal axis.

For $\alpha \geq 0$, let $J_{\alpha}$ be the halfline in CONE defined by (see Fig. 3.4)

$$
J_{\alpha}=\left\{(\alpha+\beta, \beta): \beta \in\left[-\frac{\alpha}{2}, \infty\right)\right\} .
$$

Theorem 3.1. (a) There exists a curve $\gamma \mapsto \beta_{c}(\gamma)$, lying strictly inside the upper quadrant, such that

$$
\begin{aligned}
\mathcal{L} \cap J_{\alpha} & =\left\{(\alpha+\beta, \beta): \beta \in\left(\beta_{c}(\gamma(\alpha)), \infty\right)\right\}, \\
\mathcal{D} \cap J_{\alpha} & =\left\{(\alpha+\beta, \beta): \beta \in\left[-\frac{\alpha}{2}, \beta_{c}(\gamma(\alpha))\right]\right\},
\end{aligned}
$$

for all $\alpha \in(0, \infty)$ with $\gamma(\alpha)=\alpha / \sqrt{2}$.

(b) Inside phase $\mathcal{D}$ the free energy $f$ is a function of $\alpha-\beta$ only, i.e., $f$ is constant on $J_{\alpha} \cap \mathcal{D}$ for all $\alpha \in(0, \infty)$.

3.2. Fine structure. This section is organized as follows. In Section 3.2.1, we consider the supercritical regime $p>p_{c}$, and state a theorem. Subject to two hypotheses, we show that the delocalized phase $\mathcal{D}$ (recall (3.4)) splits into two subphases $\mathcal{D}=\mathcal{D}_{1} \cup \mathcal{D}_{2}$ such that the fraction of monomers placed by the copolymer in the 
$B$ solvent is strictly positive inside $\mathcal{D}_{1}$ and equals 0 in $\mathcal{D}_{2}$. Thus, $\mathcal{D}_{1}$ and $\mathcal{D}_{2}$ are said to be non-saturated, respectively, saturated. We give a characterization of the critical curve $\alpha \mapsto \beta_{c}(\alpha)$ (recall (3.6)) in terms of the single linear free energy and state some properties of this curve. Subsequently, we formulate a conjecture stating that the localized phase $\mathcal{L}$ also splits into two subphases $\mathcal{L}=\mathcal{L}_{1} \cup \mathcal{L}_{2}$, which are non-saturated, respectively, saturated. In Section 3.2.2, we consider the subcritical regime $p<p_{c}$, and obtain similar results.

For $p \in(0,1)$ and $(\alpha, \beta) \in \mathrm{CONE}$, let $\mathcal{O}_{p, \alpha, \beta}$ denote the subset of $\overline{\mathcal{R}}_{p}$ containing those $\bar{\rho}$ that maximize the variational formula in (2.14), i.e.,

$$
\mathcal{O}_{p, \alpha, \beta}=\left\{\bar{\rho} \in \overline{\mathcal{R}}_{p}: f(\alpha, \beta ; p)=\sup _{v \in \overline{\mathcal{B}}} \frac{\bar{N}(\bar{\rho}, v)}{\bar{D}(\bar{\rho}, v)}\right\} .
$$

Throughout the remainder of this section we need the following hypothesis:

Hypothesis 3.2. For all $p \in(0,1)$ and $\alpha \in(0, \infty)$ there exists a $\bar{\rho} \in \mathcal{O}_{p, \alpha, 0}$ such that $\bar{\rho}_{\mathcal{I}}>0$.

This hypothesis will allow us to derive an expression for $\beta_{c}(\gamma)$ in (3.6).

Remark 3.3. Hypothesis 3.2 will be discussed in Section 3.3. The existence of $\bar{\rho}$ is proven in Appendix $\mathrm{F}$ for a truncated version of our model, introduced in Section 4.3. This truncated model approximates the full model as the truncation level diverges (see Proposition 6.5).

For $c \in(0, \infty)$, define $v(c)=\left(v_{A}(c), v_{B}(c), v_{\mathcal{I}}(c)\right) \in \overline{\mathcal{B}}$ as

$$
\begin{aligned}
v_{A, l}(c) & =\chi_{l}^{-1}(c), & & l \in[0, \infty), \\
v_{B, l}(c) & =\chi_{l}^{-1}\left(c+\frac{\alpha-\beta}{2}\right), & & l \in[0, \infty), \\
v_{\mathcal{I}}(c) & =z, & & \partial_{u}^{-}\left(u \phi_{\mathcal{I}}(u)\right)(z) \geq c \geq \partial_{u}^{+}\left(u \phi_{\mathcal{I}}(u)\right)(z),
\end{aligned}
$$

where

$$
\chi_{l}(v)=\left(\partial_{u}(u \tilde{\kappa}(u, l))(v)\right.
$$

and $\chi_{l}^{-1}$ denotes the inverse function. Lemma B.1(v-vi) ensures that $v \mapsto \chi_{l}(v)$ is one-to-one between $(1+l, \infty)$ and $(0, \infty)$. The existence and uniqueness of $z$ in (3.10) follow from the strict concavity of $u \mapsto u \phi_{\mathcal{I}}(u)$ (see Assumption 4.3) and Lemma C.1 (see (C.1-C.2)). We will prove in Proposition 8.1 that the maximizer $v \in \overline{\mathcal{B}}$ of (2.14) necessarily belongs to the familly $\{v(c): c \in(0, \infty)\}$.

For $\bar{\rho} \in \overline{\mathcal{R}}_{p}$, define

$$
K_{A}(\bar{\rho})=\int_{0}^{\infty}(1+l) \bar{\rho}_{A}(d l), \quad K_{B}(\bar{\rho})=\int_{0}^{\infty}(1+l) \bar{\rho}_{B}(d l) .
$$

\subsubsection{Supercritical regime.}

Splitting of the $\mathcal{D}$-phase. We partition $\mathcal{D}$ into two phases: $\mathcal{D}=\mathcal{D}_{1} \cup \mathcal{D}_{2}$. To that end we introduce the delocalized A-saturated free energy, denoted by $f_{\mathcal{D}_{2}}(p)$, which is obtained by restricting the supremum in (3.1) to those $\bar{\rho} \in \overline{\mathcal{R}}_{p}$ that do not charge $B$. Such $\bar{\rho}$, which we call $A$-saturated, exist because $p>p_{c}$, allowing for trajectories that do not visit $B$-blocks. Thus, $f_{\mathcal{D}_{2}}(p)$ is defined as

$$
f_{\mathcal{D}_{2}}(p)=\sup _{\substack{\bar{\rho} \in \overline{\mathcal{R}}_{p} \\ K_{B}(\bar{\rho})=0}} \sup _{v \in \overline{\mathcal{B}}} \frac{\bar{N}_{\mathcal{D}_{2}}(\bar{\rho}, v)}{\bar{D}_{\mathcal{D}}(\bar{\rho}, v)}
$$




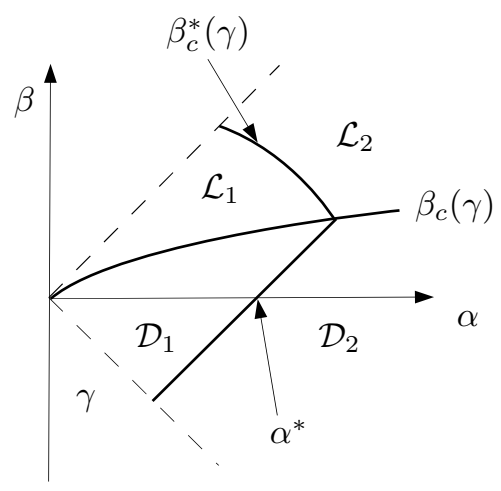

FIGURE 3.5. Qualitative picture of the phase diagram in the supercritical regime $p>p_{c}$.

with

$$
\bar{N}_{\mathcal{D}_{2}}(\bar{\rho}, v)=\int_{0}^{\infty} v_{A, l} \tilde{\kappa}\left(v_{A, l}, l\right)\left[\bar{\rho}_{A}+\bar{\rho}_{\mathcal{I}} \delta_{0}\right](d l),
$$

provided $D_{\mathcal{D}}(\bar{\rho}, v)<\infty$. Note that $f_{\mathcal{D}_{2}}(p)$ is a constant that does not depend on $(\alpha, \beta)$.

With the help of this definition, we can split the $\mathcal{D}$-phase defined in (3.4) into two parts (see Fig. 3.5):

- The $\mathcal{D}_{1}$-phase corresponds to small values of $\beta$ and small to moderate values of $\alpha$. In this phase there is no $A B$-localization and no $A$-saturation. For the variational formula in (2.14) this corresponds to the restriction where the $A B$-localization term disappears while the $A$-block term and the $B$-block term contribute, i.e.,

$$
\mathcal{D}_{1}=\left\{(\alpha, \beta) \in \mathrm{CONE}: f(\alpha, \beta ; p)=f_{\mathcal{D}}(\alpha, \beta ; p)>f_{\mathcal{D}_{2}}(p)\right\}
$$

- The $\mathcal{D}_{2}$-phase corresponds to small values of $\beta$ and large values of $\alpha$. In this phase there is no $A B$-localization but $A$-saturation occurs. For the variational formula in (2.14) this corresponds to the restriction where the $A B$-localization term disappears and the $B$-block term as well, i.e.,

$$
\mathcal{D}_{2}=\left\{(\alpha, \beta) \in \mathrm{CONE}: f(\alpha, \beta ; p)=f_{\mathcal{D}_{2}}(p)\right\} .
$$

Let $\mathcal{T}_{p}$ be the subset of $\overline{\mathcal{R}}_{p}$ containing those $\bar{\rho}$ that have a strictly positive $B$ component and are relevant for the variational formula in (2.14), i.e.,

$$
\mathcal{T}_{p}=\left\{\bar{\rho} \in \overline{\mathcal{R}}_{p}: K_{B}(\bar{\rho})>0, K_{A}(\bar{\rho})+K_{B}(\bar{\rho})<\infty\right\} .
$$

Note that $\mathcal{T}_{p}$ does not depend on $(\alpha, \beta)$. To state our main result for the delocalized part of the phase diagram we need the following hypothesis:

Hypothesis 3.4. For all $p>p_{c}$,

$$
\sup _{\bar{\rho} \in \mathcal{T}_{p}} \frac{\int_{0}^{\infty} g_{A}(l)\left[\bar{\rho}_{A}+\bar{\rho}_{\mathcal{I}} \delta_{0}\right](d l)}{K_{B}(\bar{\rho})}<\infty,
$$


where

$$
g_{A}(l)=\left.v_{A, l}(c)\left[\tilde{\kappa}\left(v_{A, l}(c), l\right)-c\right]\right|_{c=f_{\mathcal{D}_{2}}}
$$

with $v_{A, l}(c)$ as defined in (3.8).

Remark 3.5. Hypothesis 3.4 will allow us to show that $\mathcal{D}_{1}$ and $\mathcal{D}_{2}$ are non-empty. This hypothesis, which will be discussed further in Section 3.3, relies on the fact that, in the supercritical regime, large subcritical clusters typically have a diameter that is of the same size as their circumference.

Remark 3.6. The function $g_{A}$ has the following properties: (1) $g_{A}(0)>0 ;(2)$ $g_{A}$ is strictly decreasing on $[0, \infty) ;(3) \lim _{l \rightarrow \infty} g_{A}(l)=-\infty$. Property (2) follows from Lemma B.1(ii) and the fact that $u \mapsto u \tilde{\kappa}(u, l)$ is concave (see Lemma B.1(i)). Property (3) follows from $f_{\mathcal{D}_{2}}>0$, Lemma B.1(iv) and the fact that $v_{A, l}\left(f_{\mathcal{D}_{2}}\right) \geq 1+l$ for $l \in[0, \infty)$. Property (1) follows from property (2) because $\int_{0}^{\infty} g_{A}(l)\left[\bar{\rho}_{A}+\right.$ $\left.\bar{\rho}_{I} \delta_{0}\right](d l)=0$ for all $\rho$ maximizing $(3.13)$.

Let

$$
\alpha^{*}=\sup \left\{\alpha \geq 0: f_{\mathcal{D}}(\alpha, 0 ; p)>f_{\mathcal{D}_{2}}(p)\right\} \text {. }
$$

Theorem 3.7. Assume Hypotheses 3.2 and 3.4. Then the following hold:

(a) $\alpha^{*} \in(0, \infty)$.

(b) For every $\alpha \in\left[0, \alpha^{*}\right)$,

$$
J_{\alpha} \cap \mathcal{D}_{1}=J_{\alpha} \cap \mathcal{D}=\left\{(\alpha+\beta, \beta): \beta \in\left[-\frac{\alpha}{2}, \beta_{c}(\gamma(\alpha))\right] .\right.
$$

(c) For every $\alpha \in\left[\alpha^{*}, \infty\right)$,

$$
J_{\alpha} \cap \mathcal{D}_{2}=J_{\alpha} \cap \mathcal{D}=\left\{(\alpha+\beta, \beta): \beta \in\left[-\frac{\alpha}{2}, \beta_{c}(\gamma(\alpha))\right]\right\} .
$$

(d) For every $\alpha \in[0, \infty)$,

$\beta_{c}(\gamma(\alpha))=\inf \left\{\beta>0: \phi_{\mathcal{I}}\left(\bar{v}_{A, 0} ; \alpha+\beta, \beta\right)>\tilde{\kappa}\left(\bar{v}_{A, 0}, 0\right)\right\} \quad$ with $\bar{v}=v\left(f_{\mathcal{D}}(\alpha, 0 ; p)\right)$.

(e) On $\left[\alpha^{*}, \infty\right), \alpha \mapsto \beta_{c}(\gamma(\alpha))$ is concave, continuous, non-decreasing and bounded from above.

(f) Inside phase $\mathcal{D}_{1}$ the free energy $f$ is a function of $\alpha-\beta$ only, i.e., $f$ is constant on $J_{\alpha} \cap \mathcal{D}_{1}$ for all $\alpha \in\left[0, \alpha^{*}\right]$.

(g) Inside phase $\mathcal{D}_{2}$ the free energy $f$ is constant.

Splitting of the $\mathcal{L}$-phase. We partition $\mathcal{L}$ into two phases: $\mathcal{L}=\mathcal{L}_{1} \cup \mathcal{L}_{2}$. To that end we introduce the localized A-saturated free energy, denoted by $f_{\mathcal{L}_{2}}$, which is obtained by restricting the supremum in (2.14) to those $\bar{\rho} \in \overline{\mathcal{R}}_{p}$ that do not charge $B$, i.e.,

$$
f_{\mathcal{L}_{2}}(\alpha, \beta ; p)=\sup _{\substack{\bar{\rho} \in \overline{\mathcal{R}}_{p} \\ K_{B}(\bar{\rho})=0}} \sup _{v \in \overline{\mathcal{B}}} \frac{\bar{N}(\bar{\rho}, v)}{\bar{D}(\bar{\rho}, v)},
$$

provided $D(\bar{\rho}, v)<\infty$.

With the help of this definition, we can split the $\mathcal{L}$-phase defined in (3.4) into two parts (see Fig. 3.5): 
- The $\mathcal{L}_{1}$-phase corresponds to small to moderate values of $\alpha$ and large values of $\beta$. In this phase $A B$-localization occurs, but $A$-saturation does not, so that the free energy is given by the variational formula in (2.14) without restrictions, i.e.,

$$
\mathcal{L}_{1}=\left\{(\alpha, \beta) \in \mathrm{CONE}: f(\alpha, \beta ; p)>\max \left\{f_{\mathcal{L}_{2}}(\alpha, \beta ; p), f_{\mathcal{D}}(\alpha, \beta ; p)\right\}\right\} .
$$

- The $\mathcal{L}_{2}$-phase corresponds to large values of $\alpha$ and $\beta$. In this phase both $A B$-localization and $A$-saturation occur. For the variational formula in (2.14) this corresponds to the restriction where the contribution of $B$-blocks disappears, i.e.,

$$
\mathcal{L}_{2}=\left\{(\alpha, \beta) \in \mathrm{CONE}: f(\alpha, \beta ; p)=f_{\mathcal{L}_{2}}(\alpha, \beta ; p)>f_{\mathcal{D}}(\alpha, \beta ; p)\right\} .
$$

Conjecture 3.8. (a) There exists a curve $\gamma \mapsto \beta_{c}^{*}(\gamma)$, lying above the curve $\gamma \mapsto$ $\beta_{c}(\gamma)$, such that

$$
\begin{aligned}
& \mathcal{L}_{1} \cap J_{\alpha}=\left\{(\alpha+\beta, \beta): \beta \in\left(\beta_{c}(\gamma(\alpha)), \beta_{c}^{*}(\gamma(\alpha))\right]\right\} \\
& \mathcal{L}_{2} \cap J_{\alpha}=\left\{(\alpha+\beta, \beta): \beta \in\left[\beta_{c}^{*}(\gamma(\alpha)), \infty\right)\right\} .
\end{aligned}
$$

for all $\alpha \in\left(0, \alpha^{*}\right]$.

(b) $\mathcal{L}_{1} \cap J_{\alpha}=\emptyset$ for all $\alpha \in\left(\alpha^{*}, \infty\right)$.

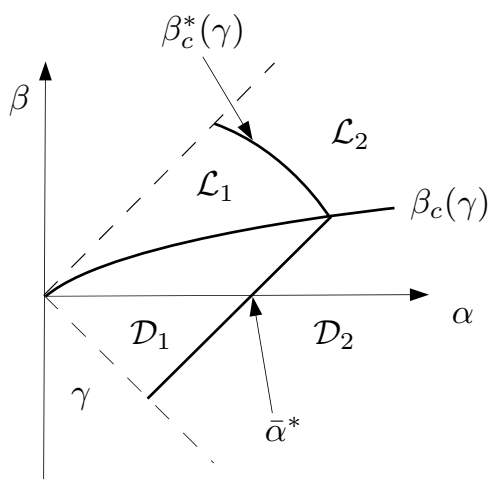

FIgURE 3.6. Qualitative picture of the phase diagram in the subcritical regime $p<p_{c}$.

3.2.2. Subcritical regime.

Splitting of the $\mathcal{D}$-phase. Let

$$
K_{p}=\inf _{\bar{\rho} \in \overline{\mathcal{R}}_{p}} K_{B}(\bar{\rho})
$$

Note that $K_{p}>0$ because $p<p_{c}$. We again partition $\mathcal{D}$ into two phases: $\mathcal{D}=$ $\mathcal{D}_{1} \cup \mathcal{D}_{2}$. To that end we introduce the delocalized maximally A-saturated free 
energy, denoted by $f_{\mathcal{D}_{2}}(p)$, which is obtained by restricting the supremum in (3.1) to those $\bar{\rho} \in \overline{\mathcal{R}}_{p}$ achieving $K_{p}$. Thus, $f_{\mathcal{D}_{2}}(p)$ is defined as

$$
f_{\mathcal{D}_{2}}(\alpha, \beta ; p)=\sup _{\substack{\bar{\rho} \in \overline{\mathcal{R}}_{p} \\ K_{B}(\bar{\rho})=K_{p}}} \sup _{v \in \overline{\mathcal{B}}} \frac{\bar{N}_{\mathcal{D}}(\bar{\rho}, v)}{\overline{D_{\mathcal{D}}(\bar{\rho}, v)}},
$$

provided $D_{\mathcal{D}}(\bar{\rho}, v)<\infty$. Note that, contrary to what we had in the supercritical regime, $f_{\mathcal{D}_{2}}(\alpha, \beta ; p)$ depends on $\alpha-\beta$.

With the help of this definition, we can split the $\mathcal{D}$-phase defined in (3.4) into two parts (see Fig. 3.6):

- The $\mathcal{D}_{1}$-phase corresponds to small values of $\beta$ and small to moderate values of $\alpha$. In this phase there is no $A B$-localization and no maximal $A$ saturation. For the variational formula in (2.14) this corresponds to the restriction where the $A B$-localization term disappears while the $A$-block term and the $B$-block term contribute, i.e.,

$$
\mathcal{D}_{1}=\left\{(\alpha, \beta) \in \mathrm{CONE}: f(\alpha, \beta ; p)=f_{\mathcal{D}}(\alpha, \beta ; p)>f_{\mathcal{D}_{2}}(p)\right\} .
$$

- The $\mathcal{D}_{2}$-phase corresponds to small values of $\beta$ and large values of $\alpha$. In this phase there is no $A B$-localization and maximal $A$-saturation. For the variational formula in (2.14) this corresponds to the restriction where the $A B$-localization term disappears and the $B$-block term is minimal, i.e.,

$$
\mathcal{D}_{2}=\left\{(\alpha, \beta) \in \mathrm{CONE}: f(\alpha, \beta ; p)=f_{\mathcal{D}_{2}}(p)\right\} .
$$

Let

$$
\mathcal{T}_{p}=\left\{\bar{\rho} \in \overline{\mathcal{R}}_{p}: K_{B}(\bar{\rho})>K_{p}, K_{A}(\bar{\rho})+K_{B}(\bar{\rho})<\infty\right\} .
$$

To state our main result for the delocalized part of the phase diagram we need the following hypothesis:

Hypothesis 3.9. For all $p>p_{c}$,

$$
\sup _{\bar{\rho} \in \mathcal{T}_{p}} \frac{\int_{0}^{\infty} g_{A, \alpha-\beta}(l)\left[\bar{\rho}_{A}+\bar{\rho}_{\mathcal{I}} \delta_{0}\right](d l)}{K_{B}(\bar{\rho})}<\infty,
$$

where

$$
g_{A, \alpha-\beta}(l)=\left.v_{A, l}(c)\left[\tilde{\kappa}\left(v_{A, l}(c), l\right)-c\right]\right|_{c=f_{\mathcal{D}_{2}}(\alpha-\beta)}
$$

with $v_{A, l}(c)$ as defined in (3.8).

Remark 3.10. Hypothesis 3.9 will allow us to show that $\mathcal{D}_{1}$ and $\mathcal{D}_{2}$ are non-empty. It is close in spirit to Hypothesis 3.4 and will be discussed further in Section 3.3.

Let

$$
\bar{\alpha}^{*}=\inf \left\{\alpha \geq 0: \forall \alpha^{\prime} \geq \alpha \exists \bar{\rho} \in \mathcal{O}_{p, \alpha^{\prime}, 0}: K_{B}(\bar{\rho})=K_{p}\right\} .
$$

Theorem 3.11. Assume Hypotheses 3.2 and 3.9 hold. Then the following hold:

(a) $\bar{\alpha}^{*} \in(0, \infty)$.

(b) Theorems $3.7(b, c, d)$ hold with $\alpha^{*}$ replaced by $\bar{\alpha}^{*}$.

(c) Theorem 3.7(f) holds on the whole $\mathcal{D}$ whereas Theorem $3.7(g)$ does not hold. 
Splitting of the $\mathcal{L}$-phase. We again partition $\mathcal{L}$ into two phases: $\mathcal{L}=\mathcal{L}_{1} \cup \mathcal{L}_{2}$. To that end we introduce the localized maximally $A$-saturated free energy, denoted by $f_{\mathcal{L}_{2}}$, which is obtained by restricting the supremum in (2.14) to those $\bar{\rho} \in \overline{\mathcal{R}}_{p}$ achieving $K_{p}$. Thus, $f_{\mathcal{L}_{2}}(\alpha, \beta ; p)$ is defined as

$$
f_{\mathcal{L}_{2}}(\alpha, \beta ; p)=\sup _{\substack{\bar{\rho} \in \overline{\mathcal{T}}_{p} \\ K_{B}(\bar{\rho})=K_{p}}} \sup _{v \in \overline{\mathcal{B}}} \frac{\bar{N}(\bar{\rho}, v)}{\bar{D}(\bar{\rho}, v)},
$$

provided $D(\bar{\rho}, v)<\infty$.

With the help of this definition, we can split the $\mathcal{L}$-phase defined in (3.4) into two parts (see Fig. 3.6):

- The $\mathcal{L}_{1}$-phase corresponds to small and moderate values of $\alpha$ and large values of $\beta$. In this phase $A B$-localization occurs, but maximal $A$-saturation does not, so that the free energy is given by the variational formula in (2.14) without restrictions, i.e.,

$$
\mathcal{L}_{1}=\left\{(\alpha, \beta) \in \mathrm{CONE}: f(\alpha, \beta ; p)>\max \left\{f_{\mathcal{L}_{2}}(\alpha, \beta ; p), f_{\mathcal{D}}(\alpha, \beta ; p)\right\}\right\} \text {. }
$$

- The $\mathcal{L}_{2}$-phase corresponds to large values of $\alpha$ and $\beta$. In this phase both $A B$-localization and maximal $A$-saturation occur. For the variational formula in (2.14) this corresponds to the restriction where the contribution of $B$-blocks is minimal, i.e.,

$$
\mathcal{L}_{2}=\left\{(\alpha, \beta) \in \mathrm{CONE}: f(\alpha, \beta ; p)=f_{\mathcal{L}_{2}}(\alpha, \beta ; p)>f_{\mathcal{D}}(\alpha, \beta ; p)\right\} .
$$

Conjecture 3.12. Conjecture 3.8 holds with $\bar{\alpha}^{*}$ instead of $\alpha^{*}$.

\subsection{Heuristics in support of the hypotheses.}

Hypothesis 3.2. At $(\alpha, 0) \in \mathrm{CONE}$, the $B B$-interaction vanishes while the $A A$ interaction does not, and we have seen earlier that there is no localization of the copolymer along $A B$-interfaces when $\beta=0$. Consequently, when the copolymer moves at a non-zero slope $l \in \mathbb{R} \backslash\{0\}$ it necessarily reduces the time it spends in the $B$-solvent. To be more specific, let $\bar{\rho} \in \overline{\mathcal{R}}_{p}$ be a maximizer of the variational formula in (2.14), and assume that the copolymer moves in the emulsion by following the strategy of displacement associated with $\bar{\rho}$. Consider the situation in which the copolymer moves upwards for awhile at slope $l>0$ and over a horizontal distance $h>0$, and subsequently changes direction to move downward at slope $l^{\prime}<0$ and over a horizontal distance $h^{\prime}>0$. This change of vertical direction is necessary to pass over a $B$-block, otherwise it would be entropically more advantageous to move at slope $\left(h l+h^{\prime} l^{\prime}\right) /\left(h+h^{\prime}\right)$ over a horizontal distance $h+h^{\prime}$ (by the strict concavity of $\tilde{\kappa}$ in Lemma B.1(i)). Next, we observe (see Fig. 3.7) that when the copolymer passes over a $B$-block, the best strategy in terms of entropy is to follow the $A B$-interface (consisting of this $B$-block and the $A$-solvent above it) without being localized, i.e., the copolymer performs a long excursion into the $A$-solvent but the two ends of this excursion are located on the $A B$-interface. This long excursion is counted in $\bar{\rho}_{\mathcal{I}}$. Consequently, Hypothesis $3.2\left(\bar{\rho}_{\mathcal{I}}>0\right)$ will be satisfied if we can show that the copolymer necessarily spends a strictly positive fraction of its time performing such changes of vertical direction. But, by the ergodicity of $\omega$ and $\Omega$, this has to be the case.

Hypothesis 3.4. The hypothesis can be rephrased in a simpler way. Recall Remark 3.6 and note that there is an $l_{0} \in(0, \infty)$ such that $g_{A}>0$ on $\left[0, l_{0}\right)$ and 

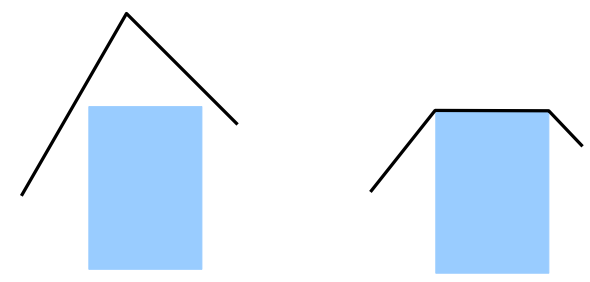

FiguRe 3.7. Entropic optimization when the copolymer passes over a $B$-block.

$g_{A}<0$ on $\left(l_{0}, \infty\right)$. Assume by contradiction that Hypothesis 3.4 fails, so that the ratio in (3.18) is unbounded. Then, by spending an arbitrarily small amount of time in the $B$-solvent, the copolymer can improve the best saturated strategies by moving some of the mass of $\bar{\rho}_{A}\left(l_{0}, \infty\right)$ to $\bar{\rho}_{A}\left(0, l_{0}\right)$, such that the entropic gain is arbitrarily larger than the time spent in the $B$-solvent. In other words, failure of Hypothesis 3.4 means that spending an arbitrarily small fraction of time in the $B$-solvent allows the copolymer to travel flatter when it is in the $A$-solvent during a fraction of the time that is arbitrarily larger than the fraction of the time it spends in the $B$-solvent. This means that, instead of going around some large cluster of the $B$-solvent, the copolymer simply crosses it straight to travel flatter. However, the fact that large subcritical clusters scale are shaped like large balls contradicts this scenario, because it means that the time needed to go around the cluster is of the same order as the time required to cross the cluster, which makes the unboundedness of the ratio in (3.18) impossible.

Hypothesis 3.9. Hypothesis 3.9 is similar to Hypothesis 3.4, except that in the subcritical regime the copolymer spends a strictly positive fraction of time in the $B$-solvent. Failure of Hypothesis 3.9 would lead to the same type of contradiction. Indeed, the unboundedness of the ratio in (3.33) would mean that there are optimal paths that spend an arbitrarily small additional fraction of time in the $B$-solvent in such a way that the path can travel flatter in the $A$-solvent during a fraction of the time that is arbitrarily larger than the fraction of the time it spends in the $B$-solvent. Again, the fact that large subcritical clusters adopt round shapes rules out such a scenario.

\section{Key ingredients}

In Section 4.1, we define the entropy per step $\tilde{\kappa}(u, l)$ carried by trajectories moving at slope $l \in \mathbb{R}_{+}$with the constraint that the total number of steps divided by the total number of horizontal steps is equal to $u \in[1+l, \infty)$ (Proposition 4.1 below). In Section 4.2 , we define the free energy per step $\phi_{\mathcal{I}}(\mu)$ of a copolymer in the vicinity of an $A B$-interface with the constraint that the total number of steps divided by the total number of horizontal steps is equal to $\mu \in[1, \infty$ ) (Proposition 4.2 below). In Section 4.3, we introduce a truncated version of the model in which we bound the vertical displacement on the block scale in each column of blocks by $M$, with $M \in \mathbb{N}$ arbitrary but fixed. (This restriction will be removed in Section 6.5 by letting $M \rightarrow \infty$.) In Section 4.4, we combine the definitions 
in Sections 4.1-4.2 to obtain a variational formula for the free energy per step in single columns of different types (Proposition 4.6 below). In Section 4.5 we define the set of probability laws introduced in (2.13), which is a key ingredient of the slope-based variational formula in Theorem 2.1. Finally, in Section 4.6, we prove that the quenched free energy per step $f(\alpha, \beta ; p)$ is strictly positive on CONE.

4.1. Path entropies at given slope.

Path entropies. We define the entropy of a path crossing a single column. To that aim, we set

$$
\begin{aligned}
\mathcal{H} & =\{(u, l) \in[0, \infty) \times \mathbb{R}: u \geq 1+|l|\}, \\
\mathcal{H}_{L} & =\left\{(u, l) \in \mathcal{H}: l \in \frac{\mathbb{Z}}{L}, u \in 1+|l|+\frac{2 \mathbb{N}}{L}\right\}, \quad L \in \mathbb{N},
\end{aligned}
$$

and note that $\mathcal{H} \cap \mathbb{Q}^{2}=\cup_{L \in \mathbb{N}} \mathcal{H}_{L}$. For $(u, l) \in \mathcal{H}$, we denote by $\mathcal{W}_{L}(u, l)$ the set containing those paths $\pi=(0,-1)+\widetilde{\pi}$ with $\tilde{\pi} \in \mathcal{W}_{u L}$ (recall (2.1)) for which $\pi_{u L}=(L, l L)$ (see Fig. 4.8). The entropy per step associated with the paths in $\mathcal{W}_{L}(u, l)$ is given by

$$
\tilde{\kappa}_{L}(u, l)=\frac{1}{u L} \log \left|\mathcal{W}_{L}(u, l)\right|
$$

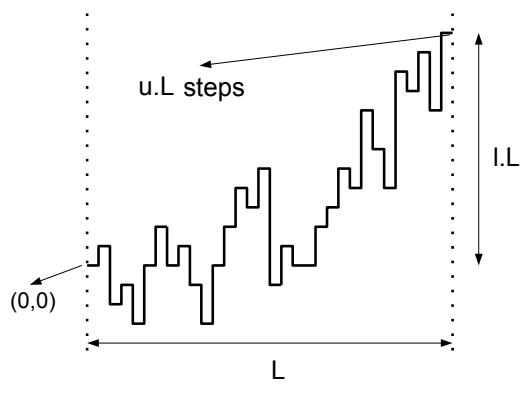

FIGURE 4.8. A trajectory in $\mathcal{W}_{L}(u, l)$.

The following propositions will be proven in Appendix A.

Proposition 4.1. For all $(u, l) \in \mathcal{H} \cap \mathbb{Q}^{2}$ there exists a $\tilde{\kappa}(u, l) \in[0, \log 3]$ such that

$$
\lim _{\substack{L \rightarrow \infty \\(u, l) \in \mathcal{H}_{L}}} \tilde{\kappa}_{L}(u, l)=\sup _{\substack{L \in \mathbb{N} \\(u, l) \in \mathcal{H}_{L}}} \tilde{\kappa}_{L}(u, l)=\tilde{\kappa}(u, l) .
$$

An explicit formula is available for $\tilde{\kappa}(u, l)$, namely,

$$
\tilde{\kappa}(u, l)= \begin{cases}\kappa(u /|l|, 1 /|l|), & l \neq 0 \\ \hat{\kappa}(u), & l=0\end{cases}
$$

where $\kappa(a, b), a \geq 1+b, b \geq 0$, and $\hat{\kappa}(\mu), \mu \geq 1$, are given in den Hollander and Whittington (2006), Section 2.1, in terms of elementary variational formulas involving entropies (see den Hollander and Whittington, 2006, proof of Lemmas 2.1.1-2.1.2). The two formulas in (4.4) allow us to extend $(u, l) \mapsto \tilde{\kappa}(u, l)$ to a continuous and strictly concave function on $\mathcal{H}$ (see Lemma B.1). 


\subsection{Free energy for a linear interface.}

Free energy along a single linear interface. To analyze the free energy per monomer in a single column we need to first analyze the free energy per monomer when the path moves in the vicinity of an $A B$-interface. To that end we consider a single linear interface $\mathcal{I}$ separating a solvent $B$ in the lower halfplane from a solvent $A$ in the upper halfplane (the latter is assumed to include the interface itself).

For $L \in \mathbb{N}$ and $\mu \in 1+\frac{2 \mathbb{N}}{L}$, let $\mathcal{W}_{L}^{\mathcal{I}}(\mu)=\mathcal{W}_{L}(\mu, 0)$ denote the set of $\mu L$-step directed self-avoiding paths starting at $(0,0)$ and ending at $(L, 0)$. Recall $(2.2)$ and define

$$
\phi_{L}^{\omega, \mathcal{I}}(\mu)=\frac{1}{\mu L} \log Z_{L, \mu}^{\omega, \mathcal{I}} \quad \text { and } \quad \phi_{L}^{\mathcal{I}}(\mu)=\mathbb{E}\left[\phi_{L}^{\omega, \mathcal{I}}(\mu)\right]
$$

with

$$
\begin{aligned}
Z_{L, \mu}^{\omega, \mathcal{I}} & =\sum_{\pi \in \mathcal{W}_{L}^{\mathcal{I}}(\mu)} \exp \left[H_{L}^{\omega, \mathcal{I}}(\pi)\right], \\
H_{L}^{\omega, \mathcal{I}}(\pi) & =\sum_{i=1}^{\mu L}\left(\beta 1\left\{\omega_{i}=B\right\}-\alpha 1\left\{\omega_{i}=A\right\}\right) 1\left\{\left(\pi_{i-1}, \pi_{i}\right)<0\right\},
\end{aligned}
$$

where $\left(\pi_{i-1}, \pi_{i}\right)<0$ means that the $i$-th step lies in the lower halfplane, strictly below the interface (see Fig. 4.9).

Proposition 4.2. (den Hollander and Whittington, 2006, Section 2.2.2)

For all $(\alpha, \beta) \in \mathrm{CONE}$ and $\mu \in \mathbb{Q} \cap[1, \infty)$ there exists a $\phi_{\mathcal{I}}(\mu)=\phi_{\mathcal{I}}(\mu ; \alpha, \beta) \in \mathbb{R}$ such that

$$
\lim _{\substack{L \rightarrow \infty \\ \mu \in 1+\frac{2 N}{L}}} \phi_{L}^{\omega, \mathcal{I}}(\mu)=\phi_{\mathcal{I}}(\mu) \quad \text { for } \mathbb{P} \text {-a.e. } \omega \text { and in } L^{1}(\mathbb{P}) .
$$

It is easy to check (with the help of concatenation of trajectories) that $\mu \mapsto$ $\mu \phi_{\mathcal{I}}(\mu ; \alpha, \beta)$ is concave. For later use we need strict concavity:

Assumption 4.3. For all $(\alpha, \beta) \in \mathrm{CONE}$ the function $\mu \mapsto \mu \phi_{\mathcal{I}}(\mu ; \alpha, \beta)$ is strictly concave on $[1, \infty)$.

This property is plausible, but hard to prove. There is to date no model of a polymer near a linear interface with disorder for which a property of this type has been established. A proof would require an explicit representation for the free energy, which for models with disorder typically is not available.

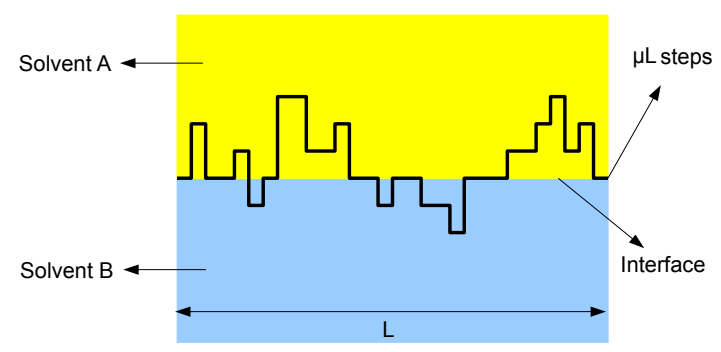

Figure 4.9. Copolymer near a single linear interface. 
4.3. Path restriction. In the remainder of this section, as well as in Sections 57, we will work with a truncation of the model in which we bound the vertical displacement on the block scale in each column of blocks by $M \in \mathbb{N}$. The value of $M$ will be arbitrary but fixed. In other words, instead of considering the full set of trajectories $\mathcal{W}_{n}$, we consider only trajectories that exit a column through a block at most $M$ above or $M$ below the block where the column was entered (see Fig. 4.10). The reason for doing the truncation is that is simplifies our proof of the column-based variational formula. In Section 6.5 we will remove the truncation by showing that the free energy of the untruncated model is the $M \rightarrow \infty$ limit of the free energy of the $M$-truncated model, and that the variational formulas match up as well.

We recall $(2.3)$ and, formally, we partition $(0, \infty) \times \mathbb{R}$ into columns of blocks of width $L_{n}$, i.e.,

$$
(0, \infty) \times \mathbb{R}=\cup_{j \in \mathbb{N}_{0}} \mathcal{C}_{j, L_{n}}, \quad \mathcal{C}_{j, L_{n}}=\cup_{k \in \mathbb{Z}} \Lambda_{L_{n}}(j, k),
$$

where $C_{j, L_{n}}$ is the $j$-th column. For each $\pi \in \mathcal{W}_{n}$, we let $\tau_{j}$ be the time at which $\pi$ leaves the $(j-1)$-th column and enters the $j$-th column, i.e.,

$\tau_{j}=\sup \left\{i \in \mathbb{N}_{0}: \pi_{i} \in \mathcal{C}_{j-1, n}\right\}=\inf \left\{i \in \mathbb{N}_{0}: \pi_{i} \in \mathcal{C}_{j, n}\right\}-1, \quad j=1, \ldots, N_{\pi}-1$,

where $N_{\pi}$ indicates how many columns have been visited by $\pi$. Finally, we let $v_{-1}(\pi)=0$ and, for $j \in\left\{0, \ldots, N_{\pi}-1\right\}$, we let $v_{j}(\pi) \in \mathbb{Z}$ be such that the block containing the last step of the copolymer in $\mathcal{C}_{j, n}$ is labelled by $\left(j, v_{j}(\pi)\right)$, i.e., $\left(\pi_{\tau_{j+1}-1}, \pi_{\tau_{j+1}}\right) \in \Lambda_{L_{N}}\left(j, v_{j}(\pi)\right)$. Thus, we restrict $\mathcal{W}_{n}$ to the subset $\mathcal{W}_{n, M}$ defined as

$$
\mathcal{W}_{n, M}=\left\{\pi \in \mathcal{W}_{n}:\left|v_{j}(\pi)-v_{j-1}(\pi)\right| \leq M \forall j \in\left\{0, \ldots, N_{\pi}-1\right\}\right\}
$$

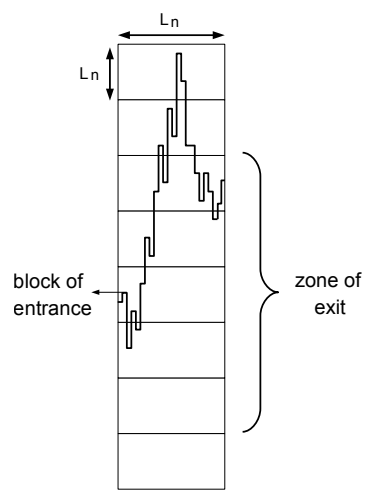

FiguRE 4.10. Example of a trajectory $\pi \in \mathcal{W}_{n, M}$ with $M=2$ crossing the column $\mathcal{C}_{0, L_{n}}$ with $v_{0}(\pi)=2$.

We recall (2.8) and we define $Z_{n, L_{n}}^{\omega, \Omega}(M ; \alpha, \beta)$ and $f_{n}^{\omega, \Omega}(M ; \alpha, \beta)$ the partition function and the quenched free energy restricted to those trajectories in $\mathcal{W}_{n, M}$, i.e.,

$f_{n}^{\omega, \Omega}(M ; \alpha, \beta)=\frac{1}{n} \log Z_{n, L_{n}}^{\omega, \Omega}(M ; \alpha, \beta)$ with $Z_{n, L_{n}}^{\omega, \Omega}(M ; \alpha, \beta)=\sum_{\pi \in \mathcal{W}_{n, M}} e^{H_{n, L_{n}}^{\omega, \Omega}(\pi ; \alpha, \beta)}$, 
and, as $n \rightarrow \infty$, the free energy per monomer is given by

$$
f(M ; \alpha, \beta)=\lim _{n \rightarrow \infty} f_{n}^{\omega, \Omega}(M ; \alpha, \beta)
$$

provided this limit exists $\omega, \Omega$-a.s.

In Remark 4.4 below we discuss how the mesoscopic vertical restriction can be relaxed by letting $M \rightarrow \infty$.

Remark 4.4. As mentioned in Section 2.3, the slope-based variational formula in Theorem 2.1 will be deduced from a column-based variational formula stated in Proposition 6.1. In this framework, the truncated model is used as follows. First, we prove the column-based variational formula for the truncated model: this will be the object of Propositions 6.2-6.4 in Section 6.1.2. Next, we show with Proposition 6.5 that, as the truncation levl $M$ diverges, the truncated free energy converges to the non-truncated free energy. This will complete the proof of the column-based variational formula for the non-truncated model. Finally, in Section 7, we transform the column-based variational formula into the slope-based variational formula for the non-truncated model.

4.4. Free energy in a single column and variational formulas. In this section, we prove the convergence of the free energy per step in a single column (Proposition 4.5) and derive a variational formula for this free energy with the help of Propositions 4.1-4.2. The variational formula takes different forms (Propositions 4.6), depending on whether there is or is not an AB-interface between the heights where the copolymer enters and exits the column, and in the latter case whether an $A B$ interface is reached or not.

In what follows we need to consider the randomness in a single column. To that aim, we recall (4.8), we pick $L \in \mathbb{N}$ and once $\Omega$ is chosen, we can record the randomness of $C_{j, L}$ as

$$
\Omega_{(j, \cdot)}=\left\{\Omega_{(j, l)}: l \in \mathbb{Z}\right\} .
$$

We will also need to consider the randomness of the $j$-th column seen by a trajectory that enters $\mathcal{C}_{j, L}$ through the block $\Lambda_{j, k}$ with $k \neq 0$ instead of $k=0$. In this case, the randomness of $\mathcal{C}_{j, L}$ is recorded as

$$
\Omega_{(j, k+\cdot)}=\left\{\Omega_{(j, k+l)}: l \in \mathbb{Z}\right\} .
$$

Pick $L \in \mathbb{N}, \chi \in\{A, B\}^{\mathbb{Z}}$ and consider $\mathcal{C}_{0, L}$ endowed with the disorder $\chi$, i.e., $\Omega(0, \cdot)=\chi$. Let $\left(n_{i}\right)_{i \in \mathbb{Z}} \in \mathbb{Z}^{\mathbb{Z}}$ be the successive heights of the $A B$-interfaces in $\mathcal{C}_{0, L}$ divided by $L$, i.e.,

$$
\cdots<n_{-1}<n_{0} \leq 0<n_{1}<n_{2}<\ldots .
$$

and the $j$-th interface of $\mathcal{C}_{0, L}$ is $\mathcal{I}_{j}=\{0, \ldots, L\} \times\left\{n_{j} L\right\}$ (see Fig. 4.11). Next, for $r \in \mathbb{N}_{0}$ we set

$$
k_{r, \chi}=0 \text { if } n_{1}>r \text { and } k_{r, \chi}=\max \left\{i \geq 1: n_{i} \leq r\right\} \text { otherwise, }
$$

while for $r \in-\mathbb{N}$ we set

$$
k_{r, \chi}=0 \text { if } n_{0} \leq r \text { and } k_{r, \chi}=\min \left\{i \leq 0: n_{i} \geq r+1\right\}-1 \text { otherwise. }
$$

Thus, $\left|k_{r, \chi}\right|$ is the number of $A B$-interfaces between heigths 1 and $r L$ in $\mathcal{C}_{0, L}$. 


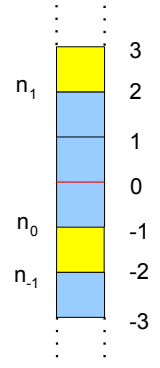

Figure 4.11. Example of a column with disorder $\chi=(\ldots, \chi(-3), \chi(-2), \chi(-1), \chi(0), \chi(1), \chi(2), \ldots)=$ $(\ldots, B, A, B, B, B, A, \ldots)$. In this example, for instance, $k_{-2, \chi}=-1$ and $k_{1, \chi}=0$.

\subsubsection{Free energy in a single column.}

Column crossing characteristics. Pick $L, M \in \mathbb{N}$, and consider the first column $\mathcal{C}_{0, L}$. The type of $\mathcal{C}_{0, L}$ is determined by $\Theta=(\chi, \Xi, x)$, where $\chi=\left(\chi_{j}\right)_{j \in \mathbb{Z}}$ encodes the type of each block in $\mathcal{C}_{0, L}$, i.e., $\chi_{j}=\Omega_{(0, j)}$ for $j \in \mathbb{Z}$, and $(\Xi, x)$ indicates which trajectories $\pi$ are taken into account. In the latter, $\Xi$ is given by $\left(\Delta \Pi, b_{0}, b_{1}\right)$ such that the vertical increment in $\mathcal{C}_{0, L}$ on the block scale is $\Delta \Pi$ and satisfies $|\Delta \Pi| \leq M$ , i.e., $\pi$ enters $\mathcal{C}_{0, L}$ at $\left(0, b_{0} L\right)$ and exits $\mathcal{C}_{0, L}$ at $\left(L,\left(\Delta \Pi+b_{1}\right) L\right)$. As in (4.16) and (4.17), we set $k_{\Theta}=k_{\Delta \Pi, \chi}$ and we let $\mathcal{V}_{\text {int }}$ be the set containing those $\Theta$ satisfying $k_{\Theta} \neq 0$. Thus, $\Theta \in \mathcal{V}_{\text {int }}$ means that the trajectories crossing $\mathcal{C}_{0, L}$ from $\left(0, b_{0} L\right)$ to $\left(L,\left(\Delta \Pi+b_{1}\right) L\right)$ necessarily hit an $A B$-interface, and in this case we set $x=1$. If, on the other hand, $\Theta \in \mathcal{V}_{\text {nint }}=\mathcal{V} \backslash \mathcal{V}_{\text {int }}$, then we have $k_{\Theta}=0$ and we set $x=1$ when the set of trajectories crossing $\mathcal{C}_{0, L}$ from $\left(0, b_{0} L\right)$ to $\left(L,\left(\Delta \Pi+b_{1}\right) L\right)$ is restricted to those that do not reach an $A B$-interface before exiting $\mathcal{C}_{0, L}$, while we set $x=2$ when it is restricted to those trajectories that reach at least one $A B$-interface before exiting $\mathcal{C}_{0, L}$. To fix the possible values taken by $\Theta=(\chi, \Xi, x)$ in a column of width $L$, we put $\mathcal{V}_{L, M}=\mathcal{V}_{\text {int }, L, M} \cup \mathcal{V}_{\text {nint }, L, M}$ with

$$
\begin{gathered}
\mathcal{V}_{\text {int }, L, M}=\left\{\left(\chi, \Delta \Pi, b_{0}, b_{1}, x\right) \in\{A, B\}^{\mathbb{Z}} \times \mathbb{Z} \times\left\{\frac{1}{L}, \frac{2}{L}, \ldots, 1\right\}^{2} \times\{1\}:\right. \\
\left.|\Delta \Pi| \leq M, k_{\Delta \Pi, \chi} \neq 0\right\}, \\
\mathcal{V}_{\text {nint }, L, M}=\left\{\left(\chi, \Delta \Pi, b_{0}, b_{1}, x\right) \in\{A, B\}^{\mathbb{Z}} \times \mathbb{Z} \times\left\{\frac{1}{L}, \frac{2}{L}, \ldots, 1\right\}^{2} \times\{1,2\}:\right. \\
\left.|\Delta \Pi| \leq M, k_{\Delta \Pi, \chi}=0\right\} .
\end{gathered}
$$

Thus, the set of all possible values of $\Theta$ is $\mathcal{V}_{M}=\cup_{L \geq 1} \mathcal{V}_{L, M}$, which we partition into $\mathcal{V}_{M}=\mathcal{V}_{\text {int }, M} \cup \mathcal{V}_{\text {nint }, M}$ (see Fig. 4.12) with

$$
\begin{aligned}
& \mathcal{V}_{\text {int }, M}=\cup_{L \in \mathbb{N}} \mathcal{V}_{\text {int }, L, M} \\
& =\left\{\left(\chi, \Delta \Pi, b_{0}, b_{1}, x\right) \in\{A, B\}^{\mathbb{Z}} \times \mathbb{Z} \times\left(\mathbb{Q}_{(0,1]}\right)^{2} \times\{1\}:|\Delta \Pi| \leq M, k_{\Delta \Pi, \chi} \neq 0\right\}, \\
& \mathcal{V}_{\text {nint }, M}=\cup_{L \in \mathbb{N}} \mathcal{V}_{\text {nint }, L, M} \\
& =\left\{\left(\chi, \Delta \Pi, b_{0}, b_{1}, x\right) \in\{A, B\}^{\mathbb{Z}} \times \mathbb{Z} \times\left(\mathbb{Q}_{(0,1]}\right)^{2} \times\{1,2\}:|\Delta \Pi| \leq M, k_{\Delta \Pi, \chi}=0\right\},
\end{aligned}
$$


where, for all $I \subset \mathbb{R}$, we set $\mathbb{Q}_{I}=I \cap \mathbb{Q}$. We define the closure of $\mathcal{V}_{M}$ as $\overline{\mathcal{V}}_{M}=$ $\overline{\mathcal{V}}_{\text {int }, M} \cup \overline{\mathcal{V}}_{\text {nint }, M}$ with

$$
\overline{\mathcal{V}}_{\text {int }, M}=\left\{\left(\chi, \Delta \Pi, b_{0}, b_{1}, x\right) \in\{A, B\}^{\mathbb{Z}} \times \mathbb{Z} \times[0,1]^{2} \times\{1\}:|\Delta \Pi| \leq M, k_{\Delta \Pi, \chi} \neq 0\right\},
$$

$\overline{\mathcal{V}}_{\text {nint }, M}=\left\{\left(\chi, \Delta \Pi, b_{0}, b_{1}, x\right) \in\{A, B\}^{\mathbb{Z}} \times \mathbb{Z} \times[0,1]^{2} \times\{1,2\}:|\Delta \Pi| \leq M, k_{\Delta \Pi, \chi}=0\right\}$.

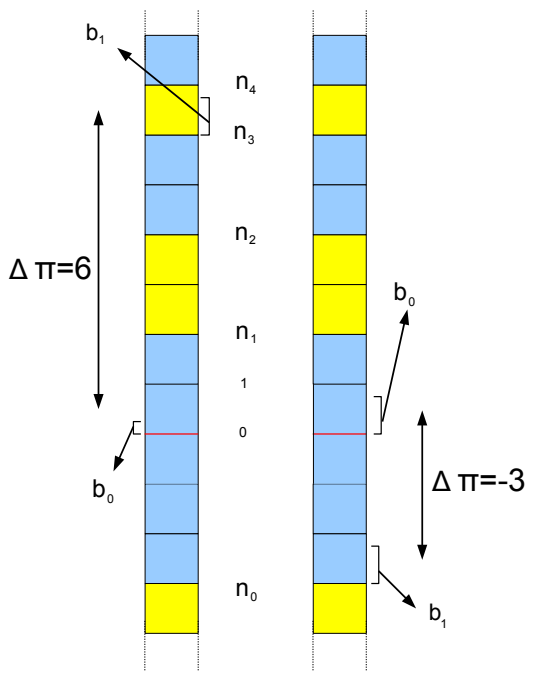

FIGURE 4.12. Labelling of coarse-grained paths and columns. On the left the type of the column is in $\mathcal{V}_{\text {int }, M}$, on the right it is in $\mathcal{V}_{\text {nint }, M}($ with $M \geq 6)$.

Time spent in columns. We pick $L, M \in \mathbb{N}, \Theta=\left(\chi, \Delta \Pi, b_{0}, b_{1}, x\right) \in \mathcal{V}_{L, M}$ and we specify the total number of steps that a trajectory crossing the column $\mathcal{C}_{0, L}$ of type $\Theta$ is allowed to make. For $\Theta=\left(\chi, \Delta \Pi, b_{0}, b_{1}, 1\right)$, set

$$
t_{\Theta}=1+\operatorname{sign}(\Delta \Pi)\left(\Delta \Pi+b_{1}-b_{0}\right) 1_{\{\Delta \Pi \neq 0\}}+\left|b_{1}-b_{0}\right| 1_{\{\Delta \Pi=0\}},
$$

so that a trajectory $\pi$ crossing a column of width $L$ from $\left(0, b_{0} L\right)$ to $\left(L,\left(\Delta \Pi+b_{1}\right) L\right)$ makes a total of $u L$ steps with $u \in t_{\Theta}+\frac{2 \mathbb{N}}{L}$. For $\Theta=\left(\chi, \Delta \Pi, b_{0}, b_{1}, 2\right)$ in turn, recall (4.15) and let

$$
t_{\Theta}=1+\min \left\{2 n_{1}-b_{0}-b_{1}-\Delta \Pi, 2\left|n_{0}\right|+b_{0}+b_{1}+\Delta \Pi\right\},
$$

so that a trajectory $\pi$ crossing a column of width $L$ and type $\Theta \in \mathcal{V}_{\text {nint }, L, M}$ from $\left(0, b_{0} L\right)$ to $\left(L,\left(\Delta \Pi+b_{1}\right) L\right)$ and reaching an $A B$-interface makes a total of $u L$ steps with $u \in t_{\Theta}+\frac{2 \mathbb{N}}{L}$.

At this stage, we can fully determine the set $\mathcal{W}_{\Theta, u, L}$ consisting of the $u L$-step trajectories $\pi$ that are considered in a column of width $L$ and type $\Theta$. To that end, for $\Theta \in \mathcal{V}_{\text {int }, L, M}$ we map the trajectories $\pi \in \mathcal{W}_{L}\left(u, \Delta \Pi+b_{1}-b_{0}\right)$ onto $\mathcal{C}_{0, L}$ such that $\pi$ enters $\mathcal{C}_{0, L}$ at $\left(0, b_{0} L\right)$ and exits $\mathcal{C}_{0, L}$ at $\left(L,\left(\Delta \Pi+b_{1}\right) L\right)$ (see Fig. 4.13), and for $\Theta \in \mathcal{V}_{\text {nint }, L, M}$ we remove, depending on $x \in\{1,2\}$, those trajectories that reach or do not reach an $A B$-interface in the column (see Fig. 4.14). Thus, for $\Theta \in \mathcal{V}_{\text {int }, L, M}$ and $u \in t_{\Theta}+\frac{2 \mathbb{N}}{L}$, we let

$$
\mathcal{W}_{\Theta, u, L}=\left\{\pi=\left(0, b_{0} L\right)+\widetilde{\pi}: \widetilde{\pi} \in \mathcal{W}_{L}\left(u, \Delta \Pi+b_{1}-b_{0}\right)\right\},
$$




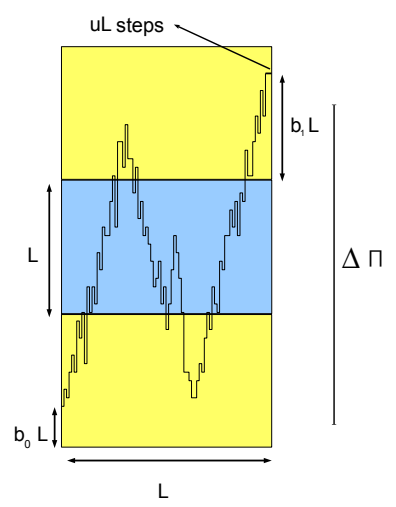

Figure 4.13. Example of a $u L$-step path inside a column of type $\left(\chi, \Delta \Pi, b_{0}, b_{1}, 1\right) \in \mathcal{V}_{\text {int, } L}$ with disorder $\chi=$ $(\ldots, \chi(0), \chi(1), \chi(2), \ldots)=(\ldots, A, B, A, \ldots)$, vertical displacement $\Delta \Pi=2$, entrance height $b_{0}$ and exit height $b_{1}$.

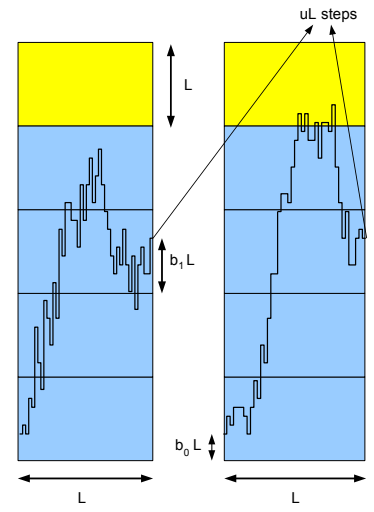

Figure 4.14. Two examples of a $u L$-step path inside a column of type $\left(\chi, \Delta \Pi, b_{0}, b_{1}, 1\right) \in \mathcal{V}_{\text {nint }, L}$ (left picture) and $\left(\chi, \Delta \Pi, b_{0}, b_{1}, 2\right) \in \mathcal{V}_{\text {nint }, L}$ (right picture) with disorder $\chi=$ $(\ldots, \chi(0), \chi(1), \chi(2), \chi(3), \chi(4), \ldots)=(\ldots, B, B, B, B, A, \ldots)$, vertical displacement $\Delta \Pi=2$, entrance height $b_{0}$ and exit height $b_{1}$.

and, for $\Theta \in \mathcal{V}_{\text {nint }, L, M}$ and $u \in t_{\Theta}+\frac{2 \mathbb{N}}{L}$,

$$
\begin{aligned}
\mathcal{W}_{\Theta, u, L}=\left\{\pi \in\left(0, b_{0} L\right)+\right. & \mathcal{W}_{L}\left(u, \Delta \Pi+b_{1}-b_{0}\right): \\
& \pi \text { reaches no } A B \text {-interface }\} \text { if } x_{\Theta}=1, \\
\mathcal{W}_{\Theta, u, L}=\left\{\pi \in\left(0, b_{0} L\right)+\right. & \mathcal{W}_{L}\left(u, \Delta \Pi+b_{1}-b_{0}\right): \\
& \pi \text { reaches an } A B \text {-interface }\} \text { if } x_{\Theta}=2,
\end{aligned}
$$


with $x_{\Theta}$ the last coordinate of $\Theta \in \mathcal{V}_{M}$. Next, we set

$$
\begin{aligned}
\mathcal{V}_{L, M}^{*} & =\left\{(\Theta, u) \in \mathcal{V}_{L, M} \times[0, \infty): u \in t_{\Theta}+\frac{2 \mathbb{N}}{L}\right\}, \\
\mathcal{V}_{M}^{*} & =\left\{(\Theta, u) \in \mathcal{V}_{M} \times \mathbb{Q}_{[1, \infty)}: u \geq t_{\Theta}\right\}, \\
\overline{\mathcal{V}}_{M}^{*} & =\left\{(\Theta, u) \in \overline{\mathcal{V}}_{M} \times[1, \infty): u \geq t_{\Theta}\right\},
\end{aligned}
$$

which we partition into $\mathcal{V}_{\text {int }, L, M}^{*} \cup \mathcal{V}_{\text {nint }, L, M}^{*}, \mathcal{V}_{\text {int }, M}^{*} \cup \mathcal{V}_{\text {nint }, M}^{*}$ and $\overline{\mathcal{V}}_{\text {int }, M}^{*} \cup \overline{\mathcal{V}}_{\text {nint }, M}^{*}$. Note that for every $(\Theta, u) \in \mathcal{V}_{M}^{*}$ there are infinitely many $L \in \mathbb{N}$ such that $(\Theta, u) \in$ $\mathcal{V}_{L, M}^{*}$, because $(\Theta, u) \in \mathcal{V}_{q L, M}^{*}$ for all $q \in \mathbb{N}$ as soon as $(\Theta, u) \in \mathcal{V}_{L, M}^{*}$.

Restriction on the number of steps per column. In what follows we abbreviate

$$
\mathrm{EIGH}=\{(M, m) \in \mathbb{N} \times \mathbb{N}: m \geq M+2\},
$$

and, for $(M, m) \in$ EIGH, we consider the situation where the number of steps $u L$ made by a trajectory $\pi$ in a column of width $L \in \mathbb{N}$ is bounded by $m L$. Thus, we restrict the set $\mathcal{V}_{L, M}$ to the subset $\mathcal{V}_{L, M}^{m}$ containing only those types of columns $\Theta$ that can be crossed in less than $m L$ steps, i.e.,

$$
\mathcal{V}_{L, M}^{m}=\left\{\Theta \in \mathcal{V}_{L, M}: t_{\Theta} \leq m\right\}
$$

Note that the latter restriction only concerns those $\Theta$ satisfying $x_{\Theta}=2$. When $x_{\Theta}=1$ a quick look at (4.21) suffices to state that $t_{\Theta} \leq M+2 \leq m$. Thus, we set $\mathcal{V}_{L, M}^{m}=\mathcal{V}_{\text {int }, L, M}^{m} \cup \mathcal{V}_{\text {nint }, L, M}^{m}$ with $\mathcal{V}_{\text {int }, L, M}^{m}=\mathcal{V}_{\text {int }, L, M}$ and with

$\mathcal{V}_{\text {nint }, L, M}^{m}=\left\{\Theta \in\{A, B\}^{\mathbb{Z}} \times \mathbb{Z} \times\left\{\frac{1}{L}, \frac{2}{L}, \ldots, 1\right\}^{2} \times\{1,2\}:\right.$

$$
\left.|\Delta \Pi| \leq M, k_{\Theta}=0 \text { and } t_{\Theta} \leq m\right\} .
$$

The sets $\mathcal{V}_{M}^{m}=\mathcal{V}_{\text {int }, M}^{m} \cup \mathcal{V}_{\text {nint }, M}^{m}$ and $\overline{\mathcal{V}}_{M}^{m}=\overline{\mathcal{V}}_{\text {int, } M}^{m} \cup \overline{\mathcal{V}}_{\text {nint }, M}^{m}$ are obtained by mimicking (4.19-4.20). In the same spirit, we restrict $\mathcal{V}_{L, M}^{*}$ to

$$
\mathcal{V}_{L, M}^{*, m}=\left\{(\Theta, u) \in \mathcal{V}_{L, M}^{*}: \Theta \in \mathcal{V}_{L, M}^{m}, u \leq m\right\}
$$

and $\mathcal{V}_{L, M}^{*}=\mathcal{V}_{\text {int }, L, M}^{*} \cup \mathcal{V}_{\text {nint }, L, M}^{*}$ with

$$
\begin{aligned}
\mathcal{V}_{\text {int }, L, M}^{*, m} & =\left\{(\Theta, u) \quad \in \mathcal{V}_{\text {int }, L, M}^{m} \times[1, m]: u \in t_{\Theta}+\frac{2 \mathbb{N}}{L}\right\}, \\
\mathcal{V}_{\text {nint }, L, M}^{* m} & =\left\{(\Theta, u) \in \mathcal{V}_{\text {nint }, L, M}^{m} \times[1, m]: u \in t_{\Theta}+\frac{2 \mathbb{N}}{L}\right\} .
\end{aligned}
$$

We set also $\mathcal{V}_{M}^{*, m}=\mathcal{V}_{\text {int }, M}^{*, m} \cup \mathcal{V}_{\text {nint }, M}^{*, m}$ with $\mathcal{V}_{\text {int }, M}^{*, m}=\cup_{L \in \mathbb{N}} \mathcal{V}_{\text {int }, L, M}^{*, m}$ and $\mathcal{V}_{\text {nint }, M}^{*, m}=$ $\cup_{L \in \mathbb{N}} \mathcal{V}_{\text {nint }, L, M}^{*, m}$, and rewrite these as

$$
\begin{aligned}
\mathcal{V}_{\mathrm{int}, M}^{*, m} & =\left\{(\Theta, u) \in \mathcal{V}_{\mathrm{int}, M}^{m} \times \mathbb{Q}_{[1, m]}: u \geq t_{\Theta}\right\}, \\
\mathcal{V}_{\text {nint }, M}^{*, m} & =\left\{(\Theta, u) \in \mathcal{V}_{\mathrm{nint}, M}^{m} \times \mathbb{Q}_{[1, m]}: u \geq t_{\Theta}\right\} .
\end{aligned}
$$

We further set $\overline{\mathcal{V}}_{M}^{*}=\overline{\mathcal{V}}_{\text {int }, M}^{*, m} \cup \overline{\mathcal{V}}_{\text {nint }, M}^{*, m}$ with

$$
\begin{aligned}
\overline{\mathcal{V}}_{\mathrm{int}, M}^{*, m} & =\left\{(\Theta, u) \in \overline{\mathcal{V}}_{\mathrm{int}, M}^{m} \times[1, m]: u \geq t_{\Theta}\right\}, \\
\overline{\mathcal{V}}_{\text {nint }, M}^{*, m} & =\left\{(\Theta, u) \in \overline{\mathcal{V}}_{\text {nint }, M}^{m} \times[1, m]: u \geq t_{\Theta}\right\} .
\end{aligned}
$$


Existence and uniform convergence of free energy per column. Recall (4.23), (4.24) and, for $L \in \mathbb{N}, \omega \in\{A, B\}^{\mathbb{N}}$ and $(\Theta, u) \in \mathcal{V}_{L, M}^{*}$, we associate with each $\pi \in \mathcal{W}_{\Theta, u, L}$ the energy

$$
H_{u L, L}^{\omega, \chi}(\pi)=\sum_{i=1}^{u L}\left(\beta 1\left\{\omega_{i}=B\right\}-\alpha 1\left\{\omega_{i}=A\right\}\right) 1\left\{\chi_{\left(\pi_{i-1}, \pi_{i}\right)}^{L}=B\right\},
$$

where $\chi_{\left(\pi_{i-1}, \pi_{i}\right)}^{L}$ indicates the label of the block containing $\left(\pi_{i-1}, \pi_{i}\right)$ in a column with disorder $\chi$ of width $L$. (Recall that the disorder in the block is part of the type of the block.) The latter allows us to define the quenched free energy per monomer in a column of type $\Theta$ and size $L$ as

$$
\psi_{L}^{\omega}(\Theta, u)=\frac{1}{u L} \log Z_{L}^{\omega}(\Theta, u) \quad \text { with } \quad Z_{L}^{\omega}(\Theta, u)=\sum_{\pi \in \mathcal{W}_{\Theta, u, L}} e^{H_{u L, L}^{\omega, \chi}(\pi)} .
$$

Abbreviate $\psi_{L}(\Theta, u)=\mathbb{E}\left[\psi_{L}^{\omega}(\Theta, u)\right]$, and note that for $M \in \mathbb{N}, m \geq M+2$ and $(\Theta, u) \in \mathcal{V}_{L, M}^{*, m}$ all $\pi \in \mathcal{W}_{\Theta, u, L}$ necessarily remain in the blocks $\Lambda_{L}(0, i)$ with $i \in$ $\{-m+1, \ldots, m-1\}$. Consequently, the dependence on $\chi$ of $\psi_{L}^{\omega}(\Theta, u)$ is restricted to those coordinates of $\chi$ indexed by $\{-m+1, \ldots, m-1\}$. The following proposition will be proven in Section 5 .

Proposition 4.5. For every $M \in \mathbb{N}$ and $(\Theta, u) \in \mathcal{V}_{M}^{*}$ there exists a $\psi(\Theta, u) \in \mathbb{R}$ such that

$$
\lim _{\substack{L \rightarrow \infty \\(\Theta, u) \in \mathcal{V}_{L}^{*}, M}} \psi_{L}^{\omega}(\Theta, u)=\psi(\Theta, u)=\psi(\Theta, u ; \alpha, \beta) \quad \omega-a . s .
$$

Moreover, for every $(M, m) \in$ EIGH the convergence is uniform in $(\Theta, u) \in \mathcal{V}_{M}^{*, m}$.

Uniform bound on the free energies. Pick $(\alpha, \beta) \in$ CONE, $n \in \mathbb{N}, \omega \in\{A, B\}^{\mathbb{N}}$, $\Omega \in\{A, B\}^{\mathbb{N}_{0} \times \mathbb{Z}}$, and let $\overline{\mathcal{W}}_{n}$ be any non-empty subset of $\mathcal{W}_{n}$ (recall (2.1)). Note that the quenched free energies per monomer introduced until now are all of the form

$$
\psi_{n}=\frac{1}{n} \log \sum_{\pi \in \overline{\mathcal{W}}_{n}} e^{H_{n}(\pi)}
$$

where $H_{n}(\pi)$ may depend on $\omega$ and $\Omega$ and satisfies $-\alpha n \leq H_{n}(\pi) \leq \alpha n$ for all $\pi \in \overline{\mathcal{W}}_{n}$ (recall that $|\beta| \leq \alpha$ in CONE). Since $1 \leq\left|\overline{\mathcal{W}}_{n}\right| \leq\left|\mathcal{W}_{n}\right| \leq 3^{n}$, we have

$$
\left|\psi_{n}\right| \leq \log 3+\alpha={ }^{\text {def }} C_{\mathrm{uf}}(\alpha) .
$$

The uniformity of this bound in $n, \omega$ and $\Omega$ allows us to average over $\omega$ and/or $\Omega$ or to let $n \rightarrow \infty$.

4.4.2. Variational formulas for the free energy in a single column. We next show how the free energies per column can be expressed in terms of a variational formula involving the path entropy and the single interface free energy defined in Sections 4.1 and 4.2. Throughout this section $M \in \mathbb{N}$ is fixed.

For $\Theta \in \overline{\mathcal{V}}_{M}$ we need to specify $l_{A, \Theta}$ and $l_{B, \Theta}$, the minimal vertical distances the copolymer must cross in blocks of type $A$ and $B$, respectively, when crossing a column of type $\Theta$. 
Vertical distance to be crossed in columns of class int. Pick $\Theta \in \overline{\mathcal{V}}_{\text {int }, M}$ and put

$$
\begin{aligned}
l_{1} & =1_{\{\Delta \Pi>0\}}\left(n_{1}-b_{0}\right)+1_{\{\Delta \Pi<0\}}\left(b_{0}-n_{0}\right), \\
l_{j} & =1_{\{\Delta \Pi>0\}}\left(n_{j}-n_{j-1}\right)+1_{\{\Delta \Pi<0\}}\left(n_{-j+2}-n_{-j+1}\right) \quad \text { for } \quad j \in\left\{2, \ldots,\left|k_{\Theta}\right|\right\}, \\
l_{\left|k_{\Theta}\right|+1} & =1_{\{\Delta \Pi>0\}}\left(\Delta \Pi+b_{1}-n_{k_{\Theta}}\right)+1_{\{\Delta \Pi<0\}}\left(n_{k_{\Theta}+1}-\Delta \Pi-b_{1}\right),
\end{aligned}
$$

i.e., $l_{1}$ is the vertical distance between the entrance point and the first interface, $l_{i}$ is the vertical distance between the $i$-th interface and the $(i+1)$-th interface, and $l_{\left|k_{\Theta}\right|+1}$ is the vertical distance between the last interface and the exit point.

Recall that $\Theta=\left(\chi, \Delta \Pi, b_{0}, b_{1}, x\right)$, and let $l_{A, \Theta}$ and $l_{B, \Theta}$ correspond to the minimal vertical distance the copolymer must cross in blocks of type $A$ and $B$, respectively, in a column with disorder $\chi$ when going from $\left(0, b_{0}\right)$ to $\left(1, \Delta \Pi+b_{1}\right)$, i.e.,

$$
\begin{aligned}
& l_{A, \Theta}=1_{\{\Delta \Pi>0\}} \sum_{j=1}^{\left|k_{\Theta}\right|+1} l_{j} 1_{\left\{\chi\left(n_{j-1}\right)=A\right\}}+1_{\{\Delta \Pi<0\}} \sum_{j=1}^{\left|k_{\Theta}\right|+1} l_{j} 1_{\left\{\chi\left(n_{-j+1}\right)=A\right\}}, \\
& l_{B, \Theta}=1_{\{\Delta \Pi>0\}} \sum_{j=1}^{\left|k_{\Theta}\right|+1} l_{j} 1_{\left\{\chi\left(n_{j-1}\right)=B\right\}}+1_{\{\Delta \Pi<0\}} \sum_{j=1}^{\left|k_{\Theta}\right|+1} l_{j} 1_{\left\{\chi\left(n_{-j+1}\right)=B\right\}} .
\end{aligned}
$$

Vertical distance to be crossed in columns of class nint. Depending on $\chi$ and $\Delta \Pi$, we further partition $\overline{\mathcal{V}}_{\text {nint }, M}$ into four parts

$$
\overline{\mathcal{V}}_{\text {nint }, A, 1, M} \cup \overline{\mathcal{V}}_{\text {nint }, A, 2, M} \cup \overline{\mathcal{V}}_{\text {nint }, B, 1, M} \cup \overline{\mathcal{V}}_{\text {nint }, B, 2, M},
$$

where $\overline{\mathcal{V}}_{\text {nint }, A, x, M}$ and $\overline{\mathcal{V}}_{\text {nint }, B, x, M}$ contain those columns with label $x$ for which all the blocks between the entrance and the exit block are of type $A$ and $B$, respectively. Pick $\Theta \in \mathcal{V}_{\text {nint, } M}$. In this case, there is no $A B$-interface between $b_{0}$ and $\Delta \Pi+b_{1}$, which means that $\Delta \Pi<n_{1}$ if $\Delta \Pi \geq 0$ and $\Delta \Pi \geq n_{0}$ if $\Delta \Pi<0\left(n_{0}\right.$ and $n_{1}$ being defined in (4.15)).

For $\Theta \in \overline{\mathcal{V}}_{\text {nint }, A, 1, M}$ we have $l_{B, \Theta}=0$, whereas $l_{A, \Theta}$ is the vertical distance between the entrance point $\left(0, b_{0}\right)$ and the exit point $\left(1, \Delta \Pi+b_{1}\right)$, i.e.,

$$
l_{A, \Theta}=1_{\{\Delta \Pi \geq 0\}}\left(\Delta \Pi-b_{0}+b_{1}\right)+1_{\{\Delta \Pi<0\}}\left(|\Delta \Pi|+b_{0}-b_{1}\right)+1_{\{\Delta \Pi=0\}}\left|b_{1}-b_{0}\right|,
$$

and similarly for $\Theta \in \overline{\mathcal{V}}_{\text {nint }, B, 1, M}$ we have obviously $l_{A, \Theta}=0$ and

$$
l_{B, \Theta}=1_{\{\Delta \Pi \geq 0\}}\left(\Delta \Pi-b_{0}+b_{1}\right)+1_{\{\Delta \Pi<0\}}\left(|\Delta \Pi|+b_{0}-b_{1}\right)+1_{\{\Delta \Pi=0\}}\left|b_{1}-b_{0}\right| .
$$

For $\Theta \in \overline{\mathcal{V}}_{\text {nint }, A, 2, M}$, in turn, we have $l_{B, \Theta}=0$ and $l_{A, \Theta}$ is the minimal vertical distance a trajectory has to cross in a column with disorder $\chi$, starting from $\left(0, b_{0}\right)$, to reach the closest $A B$-interface before exiting at $\left(1, \Delta \Pi+b_{1}\right)$, i.e.,

$$
l_{A, \Theta}=1_{\{\Delta \Pi \geq 0\}}\left(\Delta \Pi-b_{0}+b_{1}\right)+1_{\{\Delta \Pi<0\}}\left(|\Delta \Pi|+b_{0}-b_{1}\right)+1_{\{\Delta \Pi=0\}}\left|b_{1}-b_{0}\right|,
$$

and similarly for $\Theta \in \overline{\mathcal{V}}_{\text {nint }, B, 2, M}$ we have $l_{A, \Theta}=0$ and

$$
l_{B, \Theta}=1_{\{\Delta \Pi \geq 0\}}\left(\Delta \Pi-b_{0}+b_{1}\right)+1_{\{\Delta \Pi<0\}}\left(|\Delta \Pi|+b_{0}-b_{1}\right)+1_{\{\Delta \Pi=0\}}\left|b_{1}-b_{0}\right| .
$$


Variational formula for the free energy in a column. We abbreviate $(h)=$ $\left(h_{A}, h_{B}, h_{\mathcal{I}}\right)$ and $(a)=\left(a_{A}, a_{B}, a_{\mathcal{I}}\right)$. Note that the quantity $h_{x}$ indicates the fraction of horizontal steps made by the copolymer in solvent $x$ for $x \in\{A, B\}$ and along $A B$-interfaces for $x=\mathcal{I}$. Similarly, $a_{x}$ indicates the total number of steps made by the copolymer in solvent $x$ for $x \in\{A, B\}$ and along $A B$-interfaces for $x=\mathcal{I}$. For $\left(l_{A}, l_{B}\right) \in[0, \infty)^{2}$ and $u \geq l_{A}+l_{B}+1$, we put

$$
\begin{array}{r}
\mathcal{L}\left(l_{A}, l_{B} ; u\right)=\left\{(h),(a) \in[0,1]^{3} \times[0, \infty)^{3}: h_{A}+h_{B}+h_{\mathcal{I}}=1, a_{A}+a_{B}+a_{\mathcal{I}}=u\right. \\
\left.a_{A} \geq h_{A}+l_{A}, a_{B} \geq h_{B}+l_{B}, a_{\mathcal{I}} \geq h_{\mathcal{I}}\right\} .
\end{array}
$$

For $l_{A} \in[0, \infty)$ and $u \geq 1+l_{A}$, we set

$$
\begin{aligned}
& \mathcal{L}_{\text {nint }, A, 2}\left(l_{A} ; u\right)=\left\{(h),(a) \in \mathcal{L}\left(l_{A}, 0 ; u\right): h_{B}=a_{B}=0\right\}, \\
& \mathcal{L}_{\text {nint }, A, 1}\left(l_{A} ; u\right)=\left\{(h),(a) \in \mathcal{L}\left(l_{A}, 0 ; u\right): h_{B}=a_{B}=h_{\mathcal{I}}=a_{\mathcal{I}}=0\right\},
\end{aligned}
$$

and, for $l_{B} \in[0, \infty)$ and $u \geq 1+l_{B}$, we set

$$
\begin{aligned}
& \mathcal{L}_{\text {nint }, B, 2}\left(l_{B} ; u\right)=\left\{(h),(a) \in \mathcal{L}\left(0, l_{B} ; u\right): h_{A}=a_{A}=0\right\}, \\
& \mathcal{L}_{\text {nint }, B, 1}\left(l_{B} ; u\right)=\left\{(h),(a) \in \mathcal{L}\left(0, l_{B} ; u\right): h_{A}=a_{A}=h_{\mathcal{I}}=a_{\mathcal{I}}=0\right\} .
\end{aligned}
$$

The following proposition will be proved in Section 5. The free energy per step in a single column is given by the following variational formula.

Proposition 4.6. For all $\Theta \in \overline{\mathcal{V}}_{M}$ and $u \geq t_{\Theta}$,

$$
\psi(\Theta, u ; \alpha, \beta)=\sup _{(h),(a) \in \mathcal{L}(\Theta ; u)} \frac{a_{A} \tilde{\kappa}\left(\frac{a_{A}}{h_{A}}, \frac{l_{A}}{h_{A}}\right)+a_{B}\left[\tilde{\kappa}\left(\frac{a_{B}}{h_{B}}, \frac{l_{B}}{h_{B}}\right)+\frac{\beta-\alpha}{2}\right]+a_{\mathcal{I}} \phi_{\mathcal{I}}\left(\frac{a_{\mathcal{I}}}{h_{\mathcal{I}}}\right)}{u},
$$

with

$$
\begin{array}{lll}
\mathcal{L}_{\Theta, u}=\mathcal{L}\left(l_{A}, l_{B} ; u\right) & \text { if } \Theta \in \overline{\mathcal{V}}_{\mathrm{int}, M}, \\
\mathcal{L}_{\Theta, u}=\mathcal{L}_{\text {nint }, k, x}\left(l_{k} ; u\right) & \text { if } \Theta \in \overline{\mathcal{V}}_{\text {nint }, k, x, M}, k \in\{A, B\} \text { and } x \in\{1,2\} .
\end{array}
$$

The importance of Proposition 4.6 lies in the fact that it expresses the free energy in a single column in terms of the path entropy in a single column $\tilde{\kappa}$ and the free energy along a single linear interface $\phi_{\mathcal{I}}$, which were defined in Sections 4.1-4.2 and are well understood.

4.5. Mesoscopic percolation frequencies. In Section 4.5.1, we associate with each path $\pi \in \mathcal{W}_{L}$ a coarse-grained path that records the mesoscopic displacement of $\pi$ in each column. In Section 4.5.2, we define a set of probability laws providing the frequencies with which each type of column can be crossed by the copolymer. This set will be used in Section 6 to state and prove the column-based variational formula. Finally, in Section 4.5.3, we introduce a set of probability laws providing the fractions of horizontal steps that the copolymer can make when travelling inside each solvent with a given slope or along an $A B$ interface. This latter subset appears in the slope-based variational formula. 
4.5.1. Coarse-grained paths. For $x \in \mathbb{N}_{0} \times \mathbb{Z}$ and $n \in \mathbb{N}$, let $c_{x, n}$ denote the center of the block $\Lambda_{L_{n}}(x)$ defined in (2.3), i.e.,

$$
c_{x, n}=x L_{n}+\left(\frac{1}{2}, \frac{1}{2}\right) L_{n},
$$

and abbreviate

$$
\left(\mathbb{N}_{0} \times \mathbb{Z}\right)_{n}=\left\{c_{x, n}: x \in \mathbb{N}_{0} \times \mathbb{Z}\right\} .
$$

Let $\widehat{\mathcal{W}}$ be the set of coarse-grained paths on $\left(\mathbb{N}_{0} \times \mathbb{Z}\right)_{n}$ that start at $c_{0, n}$, are selfavoiding and are allowed to jump up, down and to the right between neighboring

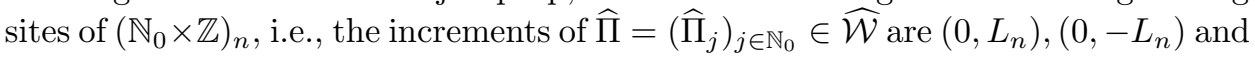
$\left(L_{n}, 0\right)$. (These paths are the coarse-grained counterparts of the paths $\pi$ introduced in (2.1).) For $l \in \mathbb{N} \cup\{\infty\}$, let $\widehat{\mathcal{W}}_{l}$ be the set of $l$-step coarse-grained paths.

Recall, for $\pi \in \mathcal{W}_{n}$, the definitions of $N_{\pi}$ and $\left(v_{j}(\pi)\right)_{j \leq N_{\pi}-1}$ given below (4.9). With $\pi$ we associate a coarse-grained path $\widehat{\Pi} \in \widehat{\mathcal{W}}_{N_{\pi}}$ that describes how $\pi$ moves with respect to the blocks. The construction of $\widehat{\Pi}$ is done as follows: $\widehat{\Pi}_{0}=c_{(0,0)}$, $\widehat{\Pi}$ moves vertically until it reaches $c_{\left(0, v_{0}\right)}$, moves one step to the right to $c_{\left(1, v_{0}\right)}$, moves vertically until it reaches $c_{\left(1, v_{1}\right)}$, moves one step to the right to $c_{\left(2, v_{1}\right)}$, and so on. The vertical increment of $\widehat{\Pi}$ in the $j$-th column is $\Delta \widehat{\Pi}_{j}=\left(v_{j}-v_{j-1}\right) L_{n}$ (see Figs. 4.12-4.14).

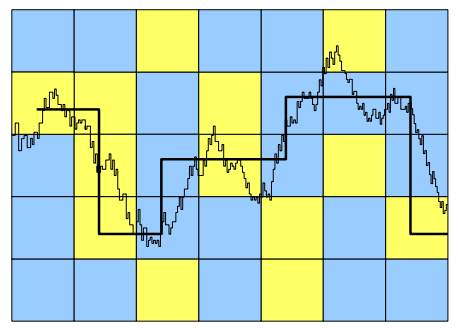

FiguRE 4.15. Example of a coarse-grained path.

To characterize a path $\pi$, we will often use the sequence of vertical increments of its associated coarse-grained path $\widehat{\Pi}$, modified in such a way that it does not depend on $L_{n}$ anymore. To that end, with every $\pi \in \mathcal{W}_{n}$ we associate $\Pi=\left(\Pi_{k}\right)_{k=0}^{N_{\pi}-1}$ such that $\Pi_{0}=0$ and,

$$
\Pi_{k}=\sum_{j=0}^{k-1} \Delta \Pi_{j} \quad \text { with } \quad \Delta \Pi_{j}=\frac{1}{L_{n}} \Delta \widehat{\Pi}_{j}, \quad j=0, \ldots, N_{\pi}-1 .
$$

Pick $M \in \mathbb{N}$ and note that $\pi \in \mathcal{W}_{n, M}$ if and only if $\left|\Delta \Pi_{j}\right| \leq M$ for all $j \in$ $\left\{0, \ldots, N_{\pi}-1\right\}$.

4.5.2. Percolation frequencies along coarse-grained paths. Given $M \in \mathbb{N}$, we denote by $\mathcal{M}_{1}\left(\overline{\mathcal{V}}_{M}\right)$ the set of probability measures on $\overline{\mathcal{V}}_{M}$. Pick $\Omega \in\{A, B\}^{\mathbb{N}_{0} \times \mathbb{Z}}, \Pi \in \mathbb{Z}^{\mathbb{N}_{0}}$ such that $\Pi_{0}=0$ and $\left|\Delta \Pi_{i}\right| \leq M$ for all $i \geq 0$ and $b=\left(b_{j}\right)_{j \in \mathbb{N}_{0}} \in\left(\mathbb{Q}_{(0,1]}\right)^{\mathbb{N}_{0}}$. Set $\Theta_{\text {traj }}=\left(\Xi_{j}\right)_{j \in \mathbb{N}_{0}}$ with

$$
\Xi_{j}=\left(\Delta \Pi_{j}, b_{j}, b_{j+1}\right), \quad j \in \mathbb{N}_{0},
$$


let

$$
\mathcal{X}_{\Pi, \Omega}=\left\{x \in\{1,2\}^{\mathbb{N}_{0}}:\left(\Omega\left(i, \Pi_{i}+\cdot\right), \Xi_{i}, x_{i}\right) \in \mathcal{V}_{M} \forall i \in \mathbb{N}_{0}\right\},
$$

and for $x \in \mathcal{X}_{\Pi, \Omega}$ set

$$
\Theta_{j}=\left(\Omega\left(j, \Pi_{j}+\cdot\right), \Delta \Pi_{j}, b_{j}, b_{j+1}, x_{j}\right), \quad j \in \mathbb{N}_{0} .
$$

With the help of (4.55), we can define the empirical distribution

$$
\rho_{N}(\Omega, \Pi, b, x)(\Theta)=\frac{1}{N} \sum_{j=0}^{N-1} 1_{\left\{\Theta_{j}=\Theta\right\}}, \quad N \in \mathbb{N}, \Theta \in \overline{\mathcal{V}}_{M} .
$$

In Appendix C.2, we define in (C.7) a distance $d$ that turns $\overline{\mathcal{V}}_{M}$ into a Polish space. Thus, the weak convergence in $\mathcal{M}_{1}\left(\overline{\mathcal{V}}_{M}\right)$ is metrizable and $\mathcal{M}_{1}\left(\overline{\mathcal{V}}_{M}\right)$ is Polish as well.

Definition 4.7. For $\Omega \in\{A, B\}^{\mathbb{N}_{0} \times \mathbb{Z}}$ and $M \in \mathbb{N}$, let

$$
\begin{aligned}
\mathcal{R}_{M, N}^{\Omega}=\{ & \rho_{N}(\Omega, \Pi, b, x) \text { with } b=\left(b_{j}\right)_{j \in \mathbb{N}_{0}} \in\left(\mathbb{Q}_{(0,1]}\right)^{\mathbb{N}_{0}}, \\
& \Pi=\left(\Pi_{j}\right)_{j \in \mathbb{N}_{0}} \in\{0\} \times \mathbb{Z}^{\mathbb{N}}:\left|\Delta \Pi_{j}\right| \leq M \quad \forall j \in \mathbb{N}_{0}, \\
& \left.x=\left(x_{j}\right)_{j \in \mathbb{N}_{0}} \in\{1,2\}^{N_{0}}:\left(\Omega\left(j, \Pi_{j}+\cdot\right), \Delta \Pi_{j}, b_{j}, b_{j+1}, x_{j}\right) \in \mathcal{V}_{M}\right\}
\end{aligned}
$$

and let $\mathcal{R}_{M}^{\Omega}$ be the set of all accumulation points of those sequences $\left(\rho_{N}\right)_{N \in \mathbb{N}}$ satisfying $\rho_{N} \in \mathcal{R}_{M, N}^{\Omega}$ for all $N \in \mathbb{N}$, i.e.,

$$
\mathcal{R}_{M}^{\Omega}=\bigcap_{N^{\prime} \in \mathbb{N}} \operatorname{closure}\left(\bigcup_{N \geq N^{\prime}} \mathcal{R}_{M, N}^{\Omega}\right),
$$

both of which are subsets of $\mathcal{M}_{1}\left(\overline{\mathcal{V}}_{M}\right)$.

Proposition 4.8. For every $p \in(0,1)$ and $M \in \mathbb{N}$ there exists a closed set $\mathcal{R}_{p, M} \subsetneq$ $\mathcal{M}_{1}\left(\overline{\mathcal{V}}_{M}\right)$ such that

$$
\mathcal{R}_{M}^{\Omega}=\mathcal{R}_{p, M} \text { for } \mathbb{P} \text {-a.e. } \Omega .
$$

Proof: Note that, for every $\Omega \in\{A, B\}^{\mathbb{N}_{0} \times \mathbb{Z}}$, the set $\mathcal{R}_{M}^{\Omega}$ does not change when finitely many variables in $\Omega$ are changed. Therefore $\mathcal{R}_{M}^{\Omega}$ is measurable with respect to the tail $\sigma$-algebra of $\Omega$. Since $\Omega$ is an i.i.d. random field, the claim follows from Kolmogorov's zero-one law. Because of the constraint on the vertical displacement, $\mathcal{R}_{p, M}$ does not coincide with $\mathcal{M}_{1}\left(\overline{\mathcal{V}}_{M}\right)$.

Each probability measure $\rho \in \mathcal{R}_{p, M}$ is associated with a strategy of displacement of the copolymer on the mesoscopic scale. As mentioned above, the growth rate of the square blocks in (2.5) ensures that no entropy is carried by the mesoscopic displacement, and this justifies the optimization over $\mathcal{R}_{p, M}$ in the column-based variational formula.

4.5.3. Fractions of horizontal steps per slope. In this section, we introduce $\overline{\mathcal{R}}_{p, M}$ as the counterpart of $\mathcal{R}_{p, M}$ for the slope-based variational formula. To that aim, we 
define

$$
\begin{aligned}
\mathcal{E}=\left\{\left(h_{A, \Theta}, h_{B, \Theta}, h_{\mathcal{I}, \Theta}\right)_{\Theta \in \overline{\mathcal{V}}_{M}} \in\left([0,1]^{3}\right)^{\overline{\mathcal{V}}_{M}}:\right. & h_{A, \Theta}+h_{B, \Theta}+h_{\mathcal{I}, \Theta}=1 \forall \Theta, \text { (4.60) } \\
& \Theta \mapsto h_{k, \Theta} \text { Borel } \forall k \in\{A, B, \mathcal{I}\}, \\
& h_{k, \Theta}>0 \text { if } l_{k, \Theta}>0 \forall k \in\{A, B\}, \\
& h_{k, \Theta}=1 \text { if } \Theta \in \mathcal{V}_{\text {nint }, k, 1, M}, \\
& \left.h_{\mathcal{I}, \Theta}+h_{k, \Theta}=1 \text { if } \Theta \in \mathcal{V}_{\text {nint }, k, 2, M}\right\} .
\end{aligned}
$$

With each $\rho \in \mathcal{R}_{p, M}$ and $h \in \mathcal{E}$ associate $G_{\rho, h} \in \mathcal{M}_{1}\left(\mathbb{R}_{+} \cup \mathbb{R}_{+} \cup\{\mathcal{I}\}\right)$, defined by

$$
\begin{aligned}
G_{\rho, h, A}(d l) & =\int_{\overline{\mathcal{V}}_{M}} h_{A, \Theta} 1\left\{\frac{l_{A, \Theta}}{h_{A, \Theta}} \in d l\right\} \rho(d \Theta), \\
G_{\rho, h, B}(d l) & =\int_{\overline{\mathcal{V}}_{M}} h_{B, \Theta} 1\left\{\frac{l_{B, \Theta}}{h_{B, \Theta}} \in d l\right\} \rho(d \Theta), \\
G_{\rho, h, \mathcal{I}} & =\int_{\overline{\mathcal{V}}_{M}} h_{\mathcal{I}, \Theta} \rho(d \Theta),
\end{aligned}
$$

where $l_{k, \Theta} / h_{k, \Theta}=0$ by convention if $h_{k, \Theta}=0$ for $\Theta \in \overline{\mathcal{V}}_{M}$ and $k \in\{A, B\}$. The set $\overline{\mathcal{R}}_{p, M}$ in (2.14) is defined as

$$
\overline{\mathcal{R}}_{p, M}=\text { Closure }\left\{\bar{\rho} \in \mathcal{M}_{1}\left(\mathbb{R}_{+} \cup \mathbb{R}_{+} \cup\{\mathcal{I}\}\right): \exists \rho \in \mathcal{R}_{p, M}, h \in \mathcal{E}: \bar{\rho}=G_{\rho, h}\right\},
$$

and as the $M$-restriction is relaxed the set $\overline{\mathcal{R}}_{p}$ in (2.14) is defined as

$$
\overline{\mathcal{R}}_{p}=\cup_{M \geq 1} \overline{\mathcal{R}}_{p, M} .
$$

For $\bar{\rho} \in \overline{\mathcal{R}}_{p}$, let $\bar{\rho}_{A}, \bar{\rho}_{B}$ and $\bar{\rho}_{\mathcal{I}}$ denote the restriction of $\bar{\rho}$ to $\mathbb{R}_{+}, \mathbb{R}_{+}$and $\{\mathcal{I}\}$, respectively, as in (2.15). The measures $\bar{\rho}_{A}(d l), \bar{\rho}_{B}(d l)$ represent the fraction of horizontal steps made by the copolymer when it moves at slope $l$ in solvent $A$, respectively, $B$. The number $\bar{\rho}_{\mathcal{I}}$ represents the fraction of horizontal steps made by the copolymer when it moves along the $A B$-interface.

4.6. Positivity of the free energy. It is easy to prove that for all $p \in(0,1), M \in \mathbb{N}$ and $(\alpha, \beta) \in$ CONE the two variational formulas (that is the slope-based variational formula stated in (2.14) but with the supremum taken over $\overline{\mathcal{R}}_{p, M}$ instead of $\overline{\mathcal{R}}_{p}$ and the column-based variational formula stated in (6.2) with the supremum taken over $\mathcal{R}_{p, M}$ instead of $\mathcal{R}_{p}$ ) are strictly positive, i.e.,

$$
f(\alpha, \beta ; M, p)>0 .
$$

To prove that the variational formula in (2.14) is strictly positive, we define $\bar{\rho}_{\text {hor }} \in \mathcal{M}_{1}\left(\mathbb{R}_{+} \cup \mathbb{R}_{+} \cup\{\mathcal{I}\}\right)$ as

$$
\bar{\rho}_{\text {hor }}=p^{2} \delta_{A, 0}(d l)+(1-p)^{2} \delta_{B, 0}(d l)+2 p(1-p) \delta_{\mathcal{I}} .
$$

When moving along the $x$-axis, the pairs of blocks appearing above and below the $x$-axis have density $p^{2}$ for type $A A$, density $(1-p)^{2}$ for type $B B$, and density $2 p(1-p)$ for types $A B$ and $B A$. Consequently, $\bar{\rho}_{\text {hor }}$ belongs to $\overline{\mathcal{R}}_{p}$ and (2.14) implies that, for any choice of $v_{A}, v_{B} \geq 1$, the variational formula in (2.14) is at least

$$
\frac{\left[p^{2}+2 p(1-p)\right] v_{A} \tilde{\kappa}\left(v_{A}, 0\right)+(1-p)^{2} v_{B}\left[\tilde{\kappa}\left(v_{B}, 0\right)+\frac{\beta-\alpha}{2}\right]}{\left[p^{2}+2 p(1-p)\right] v_{A}+(1-p)^{2} v_{B}} .
$$


Thus, it suffices to pick $v_{B}=1$, to recall that $\lim _{u \rightarrow \infty} u \tilde{\kappa}(u, 0)=\infty$ (Lemma B.1(iv)), and to choose $v_{A}$ large enough so that (4.66) becomes strictly positive.

To prove that the variational formula in (6.2) is strictly positive, we can argue similarly, taking both sequences $\left(\Pi_{i}\right)_{i \in \mathbb{N}_{0}}$ and $\left(b_{i}\right)_{i \in \mathbb{N}_{0}}$ constant and equal to 0 .

\section{Proof of Propositions 4.5-4.6}

In this section we prove Propositions 4.5 and 4.6, which were stated in Sections 4.4.1 and 4.4.2 and contain the precise definition of the key ingredients of the variational formula in Theorem 6.1. In Section 6 we will use these propositions to prove Theorem 6.1.

In Section 5.1 we associate with each trajectory $\pi$ in a column a sequence recording the indices of the $A B$-interfaces successively visited by $\pi$. The latter allows us to state a key proposition, Proposition 5.1 below, from which Propositions 4.5 and 4.6 are straightforward consequences. In Section 5.2 we give an outline of the proof of Proposition 5.1, in Sections 5.2.1-5.2.3 we provide the details.

\subsection{Column crossing characteristic.}

5.1.1. The order of the visits to the interfaces. Pick $(M, m) \in$ EIGH. To prove Propositions 4.5 and 4.6, instead of considering $(\Theta, u) \in \mathcal{V}_{M}^{*, m}$, we will restrict to $(\Theta, u) \in \mathcal{V}_{\text {int }, M}^{*, m}$. Our proof can be easily extended to $(\Theta, u) \in \mathcal{V}_{\text {nint }, M}^{*, m}$.

$\operatorname{Pick}(\Theta, u) \in \mathcal{V}_{\text {int }, M}^{*, m}$, recall (4.15) and set $\mathcal{J}_{\Theta, u}=\left\{\mathcal{N}_{\Theta, u}^{\downarrow}, \ldots, \mathcal{N}_{\Theta, u}^{\uparrow}\right\}$, with

$$
\begin{aligned}
& \mathcal{N}_{\Theta, u}^{\uparrow}=\max \left\{i \geq 1: n_{i} \leq u\right\} \quad \text { and } \quad \mathcal{N}_{\Theta, u}^{\uparrow}=0 \quad \text { if } \quad n_{1}>u . \\
& \mathcal{N}_{\Theta, u}^{\downarrow}=\min \left\{i \leq 0:\left|n_{i}\right| \leq u\right\} \quad \text { and } \quad \mathcal{N}_{\Theta, u}^{\downarrow}=1 \quad \text { if } \quad\left|n_{0}\right|>u .
\end{aligned}
$$

Next pick $L \in \mathbb{N}$ so that $(\Theta, u) \in \mathcal{V}_{\text {int }, L, M}^{*}$ and recall that for $j \in \mathcal{J}_{\Theta, u}$ the $j$-th interface of the $\Theta$-column is $\mathcal{I}_{j}=\{0, \ldots, L\} \times\left\{n_{j} L\right\}$. Note also that $\pi \in \mathcal{W}_{\Theta, u, L}$ makes $u L$ steps inside the column and therefore can not reach the $A B$-interfaces labelled outside $\left\{\mathcal{N}_{\Theta, u}^{\downarrow}, \ldots, \mathcal{N}_{\Theta, u}^{\uparrow}\right\}$.

First, we associate with each trajectory $\pi \in \mathcal{W}_{\Theta, u, L}$ the sequence $J(\pi)$ that records the indices of the interfaces that are successively visited by $\pi$. Next, we pick $\pi \in \mathcal{W}_{\Theta, u, L}$, and define $\tau_{1}, J_{1}$ as

$$
\tau_{1}=\inf \left\{i \in \mathbb{N}: \exists j \in \mathcal{J}_{\Theta, u}: \pi_{i} \in \mathcal{I}_{j}\right\}, \quad \pi_{\tau_{1}} \in \mathcal{I}_{J_{1}},
$$

so that $J_{1}=0$ (respectively, $J_{1}=1$ ) if the first interface reached by $\pi$ is $\mathcal{I}_{0}$ (respectively, $\mathcal{I}_{1}$ ). For $i \in \mathbb{N} \backslash\{1\}$, we define $\tau_{i}, J_{i}$ as

$$
\tau_{i}=\inf \left\{t>\tau_{i-1}: \exists j \in \mathcal{J}_{\Theta, u} \backslash\left\{J_{i-1}\right\}, \pi_{i} \in \mathcal{I}_{j}\right\}, \quad \pi_{\tau_{i}} \in \mathcal{I}_{J_{i}},
$$

so that the increments of $J(\pi)$ are restricted to -1 or 1 . The length of $J(\pi)$ is denoted by $m(\pi)$ and corresponds to the number of jumps made by $\pi$ between neighboring interfaces before time $u L$, i.e., $J(\pi)=\left(J_{i}\right)_{i=1}^{m(\pi)}$ with

$$
m(\pi)=\max \left\{i \in \mathbb{N}: \tau_{i} \leq u L\right\} .
$$

Note that $(\Theta, u) \in \mathcal{V}_{\text {int, } M}^{*, m}$ necessarily implies $k_{\Theta} \leq m(\pi) \leq u \leq m$. Set

$\mathcal{S}_{r}=\left\{j=\left(j_{i}\right)_{i=1}^{r} \in \mathbb{Z}^{\mathbb{N}}: j_{1} \in\{0,1\}, j_{i+1}-j_{i} \in\{-1,1\} \forall 1 \leq i \leq r-1\right\}, \quad r \in \mathbb{N}$, 
and, for $\Theta \in \mathcal{V}, r \in\{1, \ldots, m\}$ and $j \in \mathcal{S}_{r}$, define

$$
\begin{aligned}
l_{1} & =1_{\left\{j_{1}=1\right\}}\left(n_{1}-b_{0}\right)+1_{\left\{j_{1}=0\right\}}\left(b_{0}-n_{0}\right), \\
l_{i} & =\left|n_{j_{i}}-n_{j_{i-1}}\right| \text { for } i \in\{2, \ldots, r\}, \\
l_{r+1} & =1_{\left\{j_{r}=k_{\Theta}+1\right\}}\left(n_{k_{\Theta}+1}-\Delta \Pi-b_{1}\right)+1_{\left\{j_{r}=k_{\Theta}\right\}}\left(\Delta \Pi+b_{1}-n_{k_{\Theta}}\right),
\end{aligned}
$$

so that $\left(l_{i}\right)_{i \in\{1, \ldots, r+1\}}$ depends on $\Theta$ and $j$. Set

$$
\begin{aligned}
& \mathcal{A}_{\Theta, j}=\left\{i \in\{1, \ldots, r+1\}: A \text { between } \mathcal{I}_{j_{i-1}} \text { and } \mathcal{I}_{j_{i}}\right\}, \\
& \mathcal{B}_{\Theta, j}=\left\{i \in\{1, \ldots, r+1\}: B \text { between } \mathcal{I}_{j_{i-1}} \text { and } \mathcal{I}_{j_{i}}\right\},
\end{aligned}
$$

and set $l_{\Theta, j}=\left(l_{A, \Theta, j}, l_{B, \Theta, j}\right)$ with

$$
l_{A, \Theta, j}=\sum_{i \in \mathcal{A}_{\Theta, j}} l_{i}, l_{B, \Theta, j}=\sum_{i \in \mathcal{B}_{\Theta, j}} l_{i} .
$$

For $L \in \mathbb{N}$ and $(\Theta, u) \in \mathcal{V}_{\text {int }, L, M}^{*, m}$, we denote by $\mathcal{S}_{\Theta, u, L}$ the set $\left\{J(\pi), \pi \in \mathcal{W}_{\Theta, u, L}\right\}$. It is not difficult to see that a sequence $j \in \mathcal{S}_{r}$ belongs to $\mathcal{S}_{\Theta, u, L}$ if and only if it satisfies the two following conditions. First, $j_{r} \in\left\{k_{\Theta}, k_{\Theta}+1\right\}$, since $j_{r}$ is the index of the interface last visited before the $\Theta$-column is exited. Second, $u \geq 1+l_{A, \Theta, j}+l_{B, \Theta, j}$ because the number of steps taken by a trajectory $\pi \in \mathcal{W}_{\Theta, u, L}$ satisfying $J(\pi)=j$ must be large enough to ensures that all interfaces $\mathcal{I}_{j_{s}}, s \in\{1, \ldots, r\}$, can be visited by $\pi$ before time $u L$. Consequently, $\mathcal{S}_{\Theta, u, L}$ does not depend on $L$ and can be written as $\mathcal{S}_{\Theta, u}=\cup_{r=1}^{m} \mathcal{S}_{\Theta, u, r}$, where

$$
\mathcal{S}_{\Theta, u, r}=\left\{j \in \mathcal{S}_{r}: j_{r} \in\left\{k_{\Theta}, k_{\Theta}+1\right\}, u \geq 1+l_{A, \Theta, j}+l_{B, \Theta, j}\right\} .
$$

Thus, we partition $\mathcal{W}_{\Theta, u, L}$ according to the value taken by $J(\pi)$, i.e.,

$$
\mathcal{W}_{\Theta, u, L}=\bigcup_{r=1}^{m} \bigcup_{j \in \mathcal{S}_{\Theta, u, r}} \mathcal{W}_{\Theta, u, L, j},
$$

where $\mathcal{W}_{\Theta, u, L, j}$ contains those trajectories $\pi \in \mathcal{W}_{\Theta, u, L}$ for which $J(\pi)=j$.

Next, for $j \in \mathcal{S}_{\Theta, u}$, we define (recall (4.33))

$$
\psi_{L}^{\omega}(\Theta, u, j)=\frac{1}{u L} \log Z_{L}^{\omega}(\Theta, u, j), \quad \psi_{L}(\Theta, u, j)=\mathbb{E}\left[\psi_{L}^{\omega}(\Theta, u, j)\right],
$$

with

$$
Z_{L}^{\omega}(\Theta, u, j)=\sum_{\pi \in \mathcal{W}_{\Theta, u, L, j}} e^{H_{u L, L}^{\omega, \chi}(\pi)} .
$$

For each $L \in \mathbb{N}$ satisfying $(\Theta, u) \in \mathcal{V}_{\text {int }, L, M}^{*, m}$ and each $j \in \mathcal{S}_{\Theta, u}$, the quantity $l_{A, \Theta, j} L$ (respectively, $l_{B, \Theta, j} L$ ) corresponds to the minimal vertical distance a trajectory $\pi \in \mathcal{W}_{\Theta, u, L, j}$ has to cross in solvent $A$ (respectively, $B$ ).

5.1.2. Key proposition. For simplicity we give the proof for the case $(\Theta, u) \in \mathcal{V}_{\text {int, } M}^{*, m}$. The extension to $(\Theta, u) \in \mathcal{V}_{\text {nint }, M}^{*, m}$ is straightforward.

Recalling (4.48) and (5.8), we define the free energy associated with $\Theta, u, j$ as

$$
\begin{aligned}
\psi(\Theta, u, j) & =\psi_{\text {int }}\left(u, l_{\Theta, j}\right) \\
& =\sup _{(h),(u) \in \mathcal{L}\left(l_{\Theta, j} ; u\right)} \frac{u_{A} \tilde{\kappa}\left(\frac{u_{A}}{h_{A}}, \frac{l_{A, \Theta, j}}{h_{A}}\right)+u_{B}\left[\tilde{\kappa}\left(\frac{u_{B}}{h_{B}}, \frac{l_{B, \Theta, j}}{h_{B}}\right)+\frac{\beta-\alpha}{2}\right]+u_{I} \phi\left(\frac{u^{I}}{h^{I}}\right)}{u} .
\end{aligned}
$$

Proposition 5.1 below states that $\lim _{L \rightarrow \infty} \psi_{L}(\Theta, u, j)=\psi(\Theta, u, j)$ uniformly in $(\Theta, u) \in \mathcal{V}_{\text {int }, M}^{*, m}$ and $j \in \mathcal{S}_{\Theta, u}$. 
Proposition 5.1. For every $M, m \in \mathbb{N}$ such that $m \geq M+2$ and every $\varepsilon>0$ there exists an $L_{\varepsilon} \in \mathbb{N}$ such that

$$
\left|\psi_{L}(\Theta, u, j)-\psi(\Theta, u, j)\right| \leq \varepsilon \quad \forall(\Theta, u) \in \mathcal{V}_{\mathrm{int}, L, M}^{*, m}, \quad j \in \mathcal{S}_{\Theta, u}, \quad L \geq L_{\varepsilon} .
$$

Proof of Propositions 4.5 and 4.6 subject to Proposition 5.1. Pick $\varepsilon>0$, $L \in \mathbb{N}$ and $(\Theta, u) \in \mathcal{V}_{\text {int, }, L, M}^{*, m}$. Recall (4.39) and note that $l_{A}(\Theta) L$ and $l_{B}(\Theta) L$ are the minimal vertical distances the trajectories of $\mathcal{W}_{\Theta, u, L}$ have to cross in blocks of type $A$, respectively, $B$. For simplicity, in what follows the $\Theta$-dependence of $l_{A}$ and $l_{B}$ will be suppressed. In other words, $l_{A}$ and $l_{B}$ are the two coordinates of $l_{\Theta, f}$ (recall (5.8)) with $f=\left(1,2, \ldots,\left|k_{\Theta}\right|\right)$ when $\Delta \Pi \geq 0$ and $f=\left(0,-1, \ldots,-\left|k_{\Theta}\right|+1\right)$ when $\Delta \Pi<0$, so (4.48) and (5.13) imply

$$
\psi_{\mathrm{int}}\left(u, l_{A}, l_{B}\right)=\psi(\Theta, u, f) .
$$

Hence Propositions 4.5 and 4.6 will be proven once we show that $\lim _{L \rightarrow \infty} \psi_{L}(\Theta, u)=$ $\psi(\Theta, u, f)$ uniformly in $(\Theta, u) \in \mathcal{V}_{\mathrm{int}, L, M}^{*, m}$. Moreover, a look at (5.13), (5.15) and (4.48) allows us to assert that for every $j \in \mathcal{S}_{\Theta, u}$ we have $\psi(\Theta, u, j) \leq \psi(\Theta, u, f)$. The latter is a consequence of the fact that $l \mapsto \tilde{\kappa}(u, l)$ decreases on $[0, u-1]$ (see Lemma B.1(ii) in Appendix A) and that

$$
\begin{aligned}
& l_{A}=l_{A, \Theta, f}=\min \left\{l_{A, \Theta, j}: j \in \mathcal{S}_{\Theta, u}\right\}, \\
& l_{B}=l_{B, \Theta, f}=\min \left\{l_{B, \Theta, j}: j \in \mathcal{S}_{\Theta, u}\right\} .
\end{aligned}
$$

By applying Proposition 5.1 we have, for $L \geq L_{\varepsilon}$,

$$
\begin{array}{ll}
\psi_{L}(\Theta, u, j) \leq \psi(\Theta, u, f)+\varepsilon & \forall(\Theta, u) \in \mathcal{V}_{\mathrm{int}, L, M}^{*, m}, \forall j \in \mathcal{S}_{\Theta, u}, \\
\psi_{L}(\Theta, u, f) \geq \psi(\Theta, u, f)-\varepsilon & \forall(\Theta, u) \in \mathcal{V}_{\mathrm{int}, L, M}^{*, m} .
\end{array}
$$

The second inequality in (5.17) allows us to write, for $L \geq L_{\varepsilon}$,

$$
\psi(\Theta, u, f)-\varepsilon \leq \psi_{L}(\Theta, u, f) \leq \psi_{L}(\Theta, u) \quad \forall(\Theta, u) \in \mathcal{V}_{\text {int }, L, M}^{*, m} .
$$

To obtain the upper bound we introduce

$$
\mathcal{A}_{L, \varepsilon}=\left\{\omega:\left|\psi_{L}^{\omega}(\Theta, u, j)-\psi_{L}(\Theta, u, j)\right| \leq \varepsilon \quad \forall(\Theta, u) \in \mathcal{V}_{\mathrm{int}, L, M}^{*, m}, \forall j \in \mathcal{S}_{\Theta, u}\right\},
$$

so that

$$
\begin{aligned}
\psi_{L}(\Theta, u) & \leq \mathbb{E}\left[1_{\mathcal{A}_{L, \varepsilon}^{c}} \psi_{L}^{\omega}(\Theta, u)\right]+\mathbb{E}\left[1_{\mathcal{A}_{L, \varepsilon}} \psi_{L}^{\omega}(\Theta, u)\right] \\
& \leq C_{\mathrm{uf}}(\alpha) \mathbb{P}\left(\mathcal{A}_{L, \varepsilon}^{c}\right)+\frac{1}{u L} \mathbb{E}\left[1_{\mathcal{A}_{L, \varepsilon}} \log \sum_{j \in \mathcal{S}_{\Theta, u}} e^{u L\left(\psi_{L}(\Theta, u, j)+\varepsilon\right)}\right],
\end{aligned}
$$

where we use (4.37) to bound the first term in the right-hand side, and the definition of $\mathcal{A}_{L, \varepsilon}$ to bound the second term. Next, with the help of the first inequality in (5.17) we can rewrite (5.20) for $L \geq L_{\varepsilon}$ and $(\Theta, u) \in \mathcal{V}_{\text {int }, L, M}^{*, m}$ in the form

$$
\psi_{L}(\Theta, u) \leq C_{\mathrm{uf}}(\alpha) \mathbb{P}\left(\mathcal{A}_{L, \varepsilon}^{c}\right)+\frac{1}{u L} \log \left|\cup_{r=1}^{m} \mathcal{S}_{r}\right|+\psi(\Theta, u, f)+2 \varepsilon .
$$

At this stage we want to prove that $\lim _{L \rightarrow \infty} \mathbb{P}\left(\mathcal{A}_{L, \varepsilon}^{c}\right)=0$. To that end, we use the concentration of measure property in (D.3) in Appendix D with $l=u L, \Gamma=$ $\mathcal{W}_{\Theta, u, L, j}, \eta=\varepsilon u L, \xi_{i}=-\alpha 1\left\{\omega_{i}=A\right\}+\beta 1\left\{\omega_{i}=B\right\}$ for all $i \in \mathbb{N}$ and $T(x, y)=$ $1\left\{\chi_{(x, y)}^{L_{n}}=B\right\}$. We then obtain that there exist $C_{1}, C_{2}>0$ such that, for all $L \in \mathbb{N}$, $(\Theta, u) \in \mathcal{V}_{\text {int }, L, M}^{*, m}$ and $j \in \mathcal{S}_{\Theta, u}$,

$$
\mathbb{P}\left(\left|\psi_{L}^{\omega}(\Theta, u, j)-\psi_{L}(\Theta, u, j)\right|>\varepsilon\right) \leq C_{1} e^{-C_{2} \varepsilon^{2} u L} .
$$


The latter inequality, combined with the fact that $\left|\mathcal{V}_{\mathrm{int}, L, M}^{*, m}\right|$ grows polynomialy in $L$, allows us to assert that $\lim _{L \rightarrow \infty} \mathbb{P}\left(\mathcal{A}_{L, \varepsilon}^{c}\right)=0$. Next, we note that $\left|\cup_{r=1}^{m} \mathcal{S}_{r}\right|<\infty$, so that for $L_{\varepsilon}$ large enough we obtain from (5.21) that, for $L \geq L_{\varepsilon}$,

$$
\psi_{L}(\Theta, u) \leq \psi(\Theta, u, f)+3 \varepsilon \quad \forall(\Theta, u) \in \mathcal{V}_{\text {int }, L, M}^{*, m} .
$$

Now (5.18) and (5.23) are sufficient to complete the proof of Propositions 4.5-4.6 in the case $(\Theta, u) \in \mathcal{V}_{\mathrm{int}, M}^{*, m}$. As mentioned earlier, the proof for the case $(\Theta, u) \in$ $\mathcal{V}_{\text {nint }, M}^{*, m}$ is entirely similar.

\subsection{Structure of the proof of Proposition 5.1.}

Intermediate column free energies. Let

$$
G_{M}^{m}=\left\{(L, \Theta, u, j):(\Theta, u) \in \mathcal{V}_{\mathrm{int}, L, M}^{*, m}, j \in \mathcal{S}_{\Theta, u}\right\},
$$

and define the following order relation.

Definition 5.2. For $g, \widetilde{g}: G_{M}^{m} \mapsto \mathbb{R}$, write $g \prec \widetilde{g}$ when for every $\varepsilon>0$ there exists an $L_{\varepsilon} \in \mathbb{N}$ such that

$$
g(L, \Theta, u, j) \leq \widetilde{g}(L, \Theta, u, j)+\varepsilon \quad \forall(L, \Theta, u, j) \in G_{M}^{m}: L \geq L_{\varepsilon} .
$$

Recall (5.11) and (5.13), set

$$
\psi_{1}(L, \Theta, u, j)=\psi_{L}(\Theta, u, j), \quad \psi_{4}(L, \Theta, u, j)=\psi(\Theta, u, j),
$$

and note that the proof of Proposition 5.1 will be complete once we show that $\psi_{1} \prec \psi_{4}$ and $\psi_{4} \prec \psi_{1}$. In what follows, we will focus on $\psi_{1} \prec \psi_{4}$. Each step of the proof can be adapted to obtain $\psi_{4} \prec \psi_{1}$ without additional difficulty.

In the proof we need to define two intermediate free energies $\psi_{2}$ and $\psi_{3}$, in addition to $\psi_{1}$ and $\psi_{4}$ above. Our proof is divided into 3 steps, organized in Sections 5.2.1-5.2.3, and consists of showing that $\psi_{1} \prec \psi_{2} \prec \psi_{3} \prec \psi_{4}$.

Additional notation. Before stating Step 1, we need some further notation. First, we partition $\mathcal{W}_{\Theta, u, L, j}$ according to the total number of steps and the number of horizontal steps made by a trajectory along and in between $A B$-interfaces. To that end, we assume that $j \in \mathcal{S}_{\Theta, u, r}$ with $r \in\{1, \ldots, m\}$, we recall (5.6) and we let

$$
\begin{aligned}
\mathcal{D}_{\Theta, L, j} & =\left\{\left(d_{i}, t_{i}\right)_{i=1}^{r+1}: d_{i} \in \mathbb{N} \text { and } t_{i} \in d_{i}+l_{i} L+2 \mathbb{N}_{0} \forall 1 \leq i \leq r+1\right\}, \\
\mathcal{D}_{r}^{\mathcal{I}} & =\left\{\left(d_{i}^{\mathcal{I}}, t_{i}^{\mathcal{I}}\right)_{i=1}^{r}: d_{i}^{\mathcal{I}} \in \mathbb{N} \text { and } t_{i}^{\mathcal{I}} \in d_{i}^{\mathcal{I}}+2 \mathbb{N}_{0} \forall 1 \leq i \leq r\right\},
\end{aligned}
$$

where $d_{i}, t_{i}$ denote the number of horizontal steps and the total number of steps made by the trajectory between the $(i-1)$-th and $i$-th interfaces, and $d_{i}^{\mathcal{I}}, t_{i}^{\mathcal{I}}$ denote the number of horizontal steps and the total number of steps made by the trajectory along the $i$-th interface. For $(d, t) \in \mathcal{D}_{\Theta, L, j},\left(d^{\mathcal{I}}, t^{\mathcal{I}}\right) \in \mathcal{D}_{r}^{\mathcal{I}}$ and $1 \leq i \leq r$, we set $T_{0}=0$ and

$$
\begin{aligned}
V_{i} & =\sum_{j=1}^{i} t_{j}+\sum_{j=1}^{i-1} t_{j}^{\mathcal{I}}, & i & =1, \ldots, r, \\
T_{i} & =\sum_{j=1}^{i} t_{j}+\sum_{j=1}^{i} t_{j}^{\mathcal{I}}, & i & =1, \ldots, r,
\end{aligned}
$$

so that $V_{i}$, respectively, $T_{i}$ indicates the number of steps made by the trajectory when reaching, respectively, leaving the $i$-th interface. 
Next, we let $\theta: \mathbb{R}^{\mathbb{N}} \mapsto \mathbb{R}^{\mathbb{N}}$ be the left-shift acting on infinite sequences of real numbers and, for $u \in \mathbb{N}$ and $\omega \in\{A, B\}^{\mathbb{N}}$, we put

$$
H_{u}^{\omega}(B)=\sum_{i=1}^{u}\left[\beta 1_{\left\{\omega_{i}=B\right\}}-\alpha 1_{\left\{\omega_{i}=A\right\}}\right]
$$

Finally, we recall that

$$
\psi_{1}(L, \Theta, u, j)=\frac{1}{u L} \mathbb{E}\left[\log Z_{1}^{\omega}(L, \Theta, u, j)\right],
$$

where the partition function defined in (4.34) has been renamed $Z_{1}$ and can be written in the form

$$
Z_{1}^{\omega}(L, \Theta, u, j)=\sum_{(d, t) \in \mathcal{D}_{\Theta, L, j}} \sum_{\left(d^{I}, t^{\mathcal{I}}\right) \in \mathcal{D}_{r}^{I}} A_{1} B_{1} C_{1},
$$

where (recall (5.7) and (4.5))

$$
\begin{aligned}
A_{1} & =\prod_{i \in \mathcal{A}_{\Theta, j}} e^{t_{i} \tilde{\kappa}_{d_{i}}\left(\frac{t_{i}}{d_{i}}, \frac{l_{i} L}{d_{i}}\right)} \prod_{i \in \mathcal{B}_{\Theta, j}} e^{t_{i} \tilde{\kappa}_{d_{i}}\left(\frac{t_{i}}{d_{i}}, \frac{l_{i} L}{d_{i}}\right)} e^{H_{t_{i}}^{\theta^{T_{i}-1}(w)}(B)}, \\
B_{1} & =\prod_{i=1}^{r} e^{t_{i}^{\mathcal{I}} \phi_{d_{i}^{\mathcal{I}}}^{V^{V_{i}}(w)}\left(\frac{t_{i}^{\mathcal{I}}}{d_{i}^{\mathcal{T}}}\right)}, \\
C_{1} & =1_{\left\{\sum_{i=1}^{r+1} d_{i}+\sum_{i=1}^{r} d_{i}^{\mathcal{I}}=L\right\}} 1_{\left\{\sum_{i=1}^{r+1} t_{i}+\sum_{i=1}^{r} t_{i}^{\mathcal{I}}=u L\right\}} .
\end{aligned}
$$

It is important to note that a simplification has been made in the term $A_{1}$ in (5.32). Indeed, this term is not $\tilde{\kappa}_{d_{i}}(\cdot, \cdot)$ defined in $(4.2)$, since the latter does not take into account the vertical restrictions on the path when it moves from one interface to the next. However, the fact that two neighboring $A B$-interfaces are necessarily separated by a distance at least $L$ allows us to apply Lemma A.5 in Appendix A.2, which ensures that these vertical restrictions can be removed at the cost of a negligible error.

To show that $\psi_{1} \prec \psi_{2} \prec \psi_{3} \prec \psi_{4}$, we fix $(M, m) \in$ EIGH and $\varepsilon>0$, and we show that there exists an $L_{\varepsilon} \in \mathbb{N}_{s}$ such that $\psi_{k}(L, \Theta, u, j) \leq \psi_{k+1}(L, \Theta, u, j)+\varepsilon$ for all $(L, \Theta, u, j) \in G_{M}^{m}$ and $L \geq L_{\varepsilon}$. The latter will complete the proof of Proposition 5.1.

5.2.1. Step 1. In this step, we remove the $\omega$-dependence from $Z_{1}^{\omega}(L, \Theta, u, j)$. To that aim, we put

with

$$
\psi_{2}(L, \Theta, u, j)=\frac{1}{u L} \log Z_{2}(L, \Theta, u, j)
$$

$$
Z_{2}(L, \Theta, u, j)=\sum_{(d, t) \in \mathcal{D}_{\Theta, L, j}} \sum_{\left(d^{I}, t^{\mathcal{I}}\right) \in \mathcal{D}_{r}^{I}} A_{2} B_{2} C_{2},
$$

where

$$
\begin{aligned}
A_{2} & =\prod_{i \in \mathcal{A}_{\Theta, j}} e^{t_{i} \tilde{\kappa}_{d_{i}}\left(\frac{t_{i}}{d_{i}}, \frac{l_{i} L}{d_{i}}\right)} \prod_{i \in \mathcal{B}_{\Theta, j}} e^{t_{i} \tilde{\kappa}_{d_{i}}\left(\frac{t_{i}}{d_{i}}, \frac{l_{i} L}{d_{i}}\right)} e^{\frac{\beta-\alpha}{2} t_{i}}, \\
B_{2} & =\prod_{i=1}^{r} e^{t_{i}^{\mathcal{I}} \phi_{d_{i}^{\mathcal{I}}}\left(\frac{t_{i}^{\mathcal{I}}}{d_{i}^{\mathcal{T}}}\right)} \\
C_{2} & =C_{1} .
\end{aligned}
$$


Next, for $n \in \mathbb{N}$ we define

$$
\begin{aligned}
& \mathcal{A}_{\varepsilon, n}=\left\{\exists 0 \leq t, s \leq n: t \geq \varepsilon n,\left|H_{t}^{\theta^{s}(\omega)}(B)-\frac{\beta-\alpha}{2} t\right|>\varepsilon t\right\}, \\
& \mathcal{B}_{\varepsilon, n}=\left\{\exists 0 \leq t, d, s \leq n: t \in d+2 \mathbb{N}_{0}, t \geq \varepsilon n,\left|\phi_{d}^{\theta^{s}(w)}\left(\frac{t}{d}\right)-\phi_{d}\left(\frac{t}{d}\right)\right|>\varepsilon\right\} .
\end{aligned}
$$

By applying Cramér's theorem for i.i.d. random variables (see e.g. den Hollander, 2000, Chapter 1), we obtain that there exist $C_{1}(\varepsilon), C_{2}(\varepsilon)>0$ such that

$$
\mathbb{P}\left(\left|H_{t}^{\theta^{s}(w)}(B)-\frac{\beta-\alpha}{2} t\right|>\varepsilon t\right) \leq C_{1}(\varepsilon) e^{-C_{2}(\varepsilon) t}, \quad t, s \in \mathbb{N} .
$$

By using the concentration of measure property in (D.3) in Appendix D with $l=t$, $\Gamma=\mathcal{W}_{d}^{\mathcal{I}}\left(\frac{t}{d}\right), T(x, y)=1\{(x, y)<0\}, \eta=\varepsilon t$ and $\xi_{i}=-\alpha 1\left\{\omega_{i}=A\right\}+\beta 1\left\{\omega_{i}=B\right\}$ for all $i \in \mathbb{N}$, we find that there exist $C_{1}, C_{2}>0$ such that

$$
\mathbb{P}\left(\left|\phi_{d}^{\theta^{s}(w)}\left(\frac{t}{d}\right)-\phi_{d}\left(\frac{t}{d}\right)\right|>\varepsilon \mid\right) \leq C_{1} e^{-C_{2} \varepsilon^{2} t}, \quad t, d, s \in \mathbb{N}, t \in d+2 \mathbb{N}_{0} .
$$

With the help of (4.37) and (5.30) we may write, for $(L, \Theta, u, j) \in G_{M}^{m}$,

$\psi_{1}(L, \Theta, u, j) \leq C_{\mathrm{uf}}(\alpha) \mathbb{P}\left(\mathcal{A}_{\varepsilon, m L} \cup \mathcal{B}_{\varepsilon, m L}\right)+\frac{1}{u L} \mathbb{E}\left[1_{\left\{\mathcal{A}_{\varepsilon, m L}^{c} \cap \mathcal{B}_{\varepsilon, m L}^{c}\right\}} \log Z_{1}^{\omega}(L, \Theta, u, j)\right]$.

With the help of (5.37) and (5.38), we get that $\mathbb{P}\left(\mathcal{A}_{\varepsilon, m L}\right) \rightarrow 0$ and $\mathbb{P}\left(\mathcal{B}_{\varepsilon, m L}\right) \rightarrow 0$ as $L \rightarrow \infty$. Moreover, from (5.31-5.36) it follows that, for $(L, \Theta, u, j) \in G_{M}^{m}$ and $\omega \in \mathcal{A}_{\varepsilon, m L}^{c} \cap \mathcal{B}_{\varepsilon, M L}^{c}$,

$$
Z_{1}^{\omega}(L, \Theta, u, j) \leq Z_{2}(L, \Theta, u, j) e^{\varepsilon u L} .
$$

The latter completes the proof of $\psi_{1} \prec \psi_{2}$.

5.2.2. Step 2. In this step, we concatenate the pieces of trajectories that travel in $A$-blocks, respectively, $B$-blocks, respectively, along the $A B$-interfaces and replace the finite-size entropies and free energies by their infinite-size counterparts. Recall the definition of $l_{A, \Theta, j}$ and $l_{B, \Theta, j}$ in (5.8) and define, for $(L, \Theta, u, j) \in G_{M}^{m}$, the sets

$$
\begin{aligned}
\mathcal{J}_{\Theta, L, j} & =\left\{\left(a_{A}, h_{A}, a_{B}, h_{B}\right) \in \mathbb{N}^{4}: a_{A} \in l_{A, \Theta, j} L+h_{A}+2 \mathbb{N}_{0}, a_{B} \in l_{B, \Theta, j} L+h_{B}+2 \mathbb{N}_{0}\right\}, \\
\mathcal{J}^{\mathcal{I}} & =\left\{\left(a^{\mathcal{I}}, h^{\mathcal{I}}\right) \in \mathbb{N}^{2}: a^{\mathcal{I}} \in h^{\mathcal{I}}+2 \mathbb{N}_{0}\right\},
\end{aligned}
$$

and put $\psi_{3}(L, \Theta, u, j)=\frac{1}{u L} \log Z_{3}(L, \Theta, u, j)$ with

$$
Z_{3}(L, \Theta, u, j)=\sum_{(a, h) \in \mathcal{J}_{\Theta, L, j}} \sum_{\left(a^{\mathcal{I}}, h^{\mathcal{I}}\right) \in \mathcal{J}^{\mathcal{I}}} A_{3} B_{3} C_{3},
$$

where

$$
\begin{aligned}
& A_{3}=e^{a_{A} \tilde{\kappa}\left(\frac{a_{A}}{h_{A}}, \frac{l_{A, \Theta, j} L}{h_{A}}\right)} e^{a_{B} \tilde{\kappa}\left(\frac{a_{B}}{h_{B}}, \frac{l_{B, \Theta, j} L}{h_{B}}\right)} e^{\frac{\beta-\alpha}{2} a_{B}}, \\
& B_{3}=e^{a^{\mathcal{I}} \phi\left(\frac{a^{\mathcal{I}}}{h^{\mathcal{I}}}\right)}, \\
& C_{3}=1_{\left\{a_{A}+a_{B}+a^{\mathcal{I}}=u L\right\}} 1_{\left\{h_{A}+h_{B}+h^{\mathcal{I}}=L\right\}} .
\end{aligned}
$$


In order to establish a link between $\psi_{2}$ and $\psi_{3}$ we define, for $(a, h) \in \mathcal{J}_{\Theta, L, j}$ and $\left(a^{\mathcal{I}}, h^{\mathcal{I}}\right) \in \mathcal{J}^{\mathcal{I}}$

$$
\begin{aligned}
& \mathcal{P}_{(a, h)}=\left\{(t, d) \in \mathcal{D}_{\Theta, L, j}: \sum_{i \in \mathcal{A}_{\Theta, j}}\left(t_{i}, d_{i}\right)=\left(a_{A}, h_{A}\right), \sum_{i \in \mathcal{B}_{\Theta, j}}\left(t_{i}, d_{i}\right)=\left(a_{B}, h_{B}\right)\right\}, \\
& \mathcal{Q}_{\left(a^{\mathcal{I}}, h^{\mathcal{I}}\right)}=\left\{\left(t^{\mathcal{I}}, d^{\mathcal{I}}\right) \in \mathcal{D}_{r}^{\mathcal{I}}: \sum_{i=1}^{r}\left(t_{i}^{\mathcal{I}}, d_{i}^{\mathcal{I}}\right)=\left(a^{\mathcal{I}}, h^{\mathcal{I}}\right)\right\} .
\end{aligned}
$$

Then we can rewrite $Z_{2}$ as

$$
Z_{2}(L, \Theta, u, j)=\sum_{(a, h) \in \mathcal{J}_{\Theta, L, j}} \sum_{\left(a^{\mathcal{I}}, h^{\mathcal{I}}\right) \in \mathcal{J}^{\mathcal{I}}} C_{3} \sum_{(t, d) \in \mathcal{P}_{(a, h)}} \sum_{\left(t^{\mathcal{I}}, d^{\mathcal{I}}\right) \in \mathcal{Q}_{\left(a^{\mathcal{I}}, h^{\mathcal{I}}\right)}} A_{2} B_{2} .
$$

To prove that $\psi_{2} \prec \psi_{3}$, we need the following lemma.

Lemma 5.3. For every $\eta>0$ there exists an $L_{\eta} \in \mathbb{N}$ such that, for every $(L, \Theta, u, j) \in G_{M}^{m}$ with $L \geq L_{\eta}$ and every $(d, t) \in \mathcal{D}_{\Theta, L, j}$ and $\left(d^{\mathcal{I}}, t^{\mathcal{I}}\right) \in \mathcal{D}_{r}^{\mathcal{I}}$ satisfying $\sum_{i=1}^{r+1} d_{i}+\sum_{i=1}^{r} d_{i}^{\mathcal{I}}=L$ and $\sum_{i=1}^{r+1} t_{i}+\sum_{i=1}^{r} t_{i}^{\mathcal{I}}=u L$,

$$
\begin{gathered}
t_{i} \tilde{\kappa}\left(\frac{t_{i}}{d_{i}}, \frac{l_{i} L}{d_{i}}\right)-\eta u L \leq t_{i} \tilde{\kappa}_{d_{i}}\left(\frac{t_{i}}{d_{i}}, \frac{l_{i} L}{d_{i}}\right) \leq t_{i} \tilde{\kappa}\left(\frac{t_{i}}{d_{i}}, \frac{l_{i} L}{d_{i}}\right)+\eta u L \quad i=1, \ldots, r+1 \\
t_{i}^{\mathcal{I}} \phi\left(\frac{t_{i}^{\mathcal{I}}}{d_{i}^{\mathcal{I}}}\right)-\eta u L \leq t_{i}^{\mathcal{I}} \phi_{d_{i}^{\mathcal{I}}}\left(\frac{t_{i}^{\mathcal{I}}}{d_{i}^{\mathcal{I}}}\right) \leq t_{i}^{\mathcal{I}} \phi\left(\frac{t_{i}^{\mathcal{I}}}{d_{i}^{\mathcal{I}}}\right)+\eta u L \quad i=1, \ldots, r .
\end{gathered}
$$

Proof: By using Lemmas A.1 and C.2 in Appendix A, we have that there exists a $\tilde{L}_{\eta} \in \mathbb{N}$ such that, for $L \geq \tilde{L}_{\eta},(u, l) \in \mathcal{H}_{L}$ and $\mu \in 1+\frac{2 \mathbb{N}}{L}$,

$$
\left|\tilde{\kappa}_{L}(u, l)-\tilde{\kappa}(u, l)\right| \leq \eta, \quad\left|\phi_{L}^{\mathcal{I}}(\mu)-\phi^{\mathcal{I}}(\mu)\right| \leq \eta .
$$

Moreover, Lemmas 4.1, B.1(ii-iii), C.1(ii) and C.2 ensure that there exists a $v_{\eta}>1$ such that, for $L \geq 1,(u, l) \in \mathcal{H}_{L}$ with $u \geq v_{\eta}$ and $\mu \in 1+\frac{2 \mathbb{N}}{L}$ with $\mu \geq v_{\eta}$,

$$
0 \leq \tilde{\kappa}_{L}(u, l) \leq \eta, \quad 0 \leq \phi_{L}(\mu) \leq \eta
$$

Note that the two inequalities in (5.48) remain valid when $L=\infty$. Next, we set $r_{\eta}=\eta /\left(2 v_{\eta} C_{\mathrm{uf}}\right)$ and $L_{\eta}=\tilde{L}_{\eta} / r_{\eta}$, and we consider $L \geq L_{\eta}$. Because of the left-hand side of (5.47), the two inequalities in the first line of (5.46) hold when $d_{i} \geq r_{\eta} L \geq \tilde{L}_{\eta}$. We deal with the case $d_{i} \leq r_{\eta} L$ by considering first the case $t_{i} \leq \eta u L / 2 C_{\mathrm{uf}}$, which is easy because $\tilde{\kappa}_{d_{i}}$ and $\tilde{\kappa}$ are uniformly bounded by $C_{\mathrm{uf}}$ (see (4.37)). The case $t_{i} \geq \eta u L / 2 C_{\text {uf }}$ gives $t_{i} / d_{i} \geq u v_{\eta} \geq v_{\eta}$, which by the left-hand side of (5.48) completes the proof of the first line in (5.46). The same observations applied to $t_{i}^{\mathcal{I}}, d_{i}^{\mathcal{I}}$ combined with the right-hand side of (5.47) and (5.48) provide the two inequalities in the second line in (5.46).

To prove that $\psi_{2} \prec \psi_{3}$, we apply Lemma 5.3 with $\eta=\varepsilon /(2 m+1)$ and we use (5.35) to obtain, for $L \geq L_{\varepsilon /(2 m+1)},(d, t) \in \mathcal{D}_{\Theta, L, j}$ and $\left(d^{\mathcal{I}}, t^{\mathcal{I}}\right) \in \mathcal{D}_{r}^{\mathcal{I}}$,

$$
\begin{aligned}
A_{2} & \leq \prod_{i \in \mathcal{A}_{\Theta, j}} e^{t_{i} \tilde{\kappa}\left(\frac{t_{i}}{d_{i}}, \frac{l_{i} L}{d_{i}}\right)+\frac{\varepsilon u L}{2 m+1}} \prod_{i \in \mathcal{B}_{\Theta, j}} e^{t_{i} \tilde{\kappa}\left(\frac{t_{i}}{d_{i}}, \frac{l_{i} L}{d_{i}}\right)+t_{i} \frac{\beta-\alpha}{2}+\frac{\varepsilon u L}{2 m+1}}, \\
B_{2} & \leq \prod_{i=1}^{r} e^{t_{i}^{\mathcal{I}} \phi\left(\frac{t_{i}^{\mathcal{I}}}{d_{i}^{\mathcal{I}}}\right)+\frac{\varepsilon u L}{2 m+1}} .
\end{aligned}
$$

Next, we pick $(a, h) \in \mathcal{J}_{\Theta, L, j},\left(a^{\mathcal{I}}, h^{\mathcal{I}}\right) \in \mathcal{J}^{\mathcal{I}},(t, d) \in \mathcal{P}_{(a, h)}$ and $\left(t^{\mathcal{I}}, d^{\mathcal{I}}\right) \in \mathcal{Q}_{\left(a^{\mathcal{I}}, h^{\mathcal{I}}\right)}$, and we use the concavity of $(a, b) \mapsto a \tilde{\kappa}(a, b)$ and $\mu \mapsto \phi^{\mathcal{I}}(\mu)$ (see Lemma B.1 in 
Appendix A and Lemma C.1 in Appendix C) to rewrite (5.49) as

$$
\begin{aligned}
& A_{2} \leq e^{a_{A} \tilde{\kappa}\left(\frac{a_{A}}{h_{A}}, \frac{l_{A, \Theta, j} L}{h_{A}}\right)+a_{B} \tilde{\kappa}\left(\frac{a_{B}}{h_{B}}, \frac{l_{B, \Theta, j} L}{h_{B}}\right)+\frac{\beta-\alpha}{2} a_{B}+\frac{\varepsilon(r+1) u L}{2 m+1}=A_{3} e^{\frac{\varepsilon(r+1) u L}{2 m+1}},} \\
& B_{2} \leq e^{a^{\mathcal{I}} \phi^{\mathcal{I}}\left(\frac{a^{\mathcal{I}}}{h^{\mathcal{I}}}\right)+\frac{\varepsilon r u L}{2 m+1}}=B_{3} e^{\frac{\varepsilon r u L}{2 m+1}} .
\end{aligned}
$$

Moreover, $r$, which is the number of $A B$ interfaces crossed by the trajectories in $\mathcal{W}_{\Theta, u, j, L}$, is at most $m$ (see (5.10)), so that (5.50) allows us to rewrite (5.45) as

$$
Z_{2}(L, \Theta, u, j) \leq e^{\varepsilon u L} \sum_{(a, h) \in \mathcal{J}_{\Theta, L, j}} \sum_{\left(a^{\mathcal{I}}, h^{\mathcal{I}}\right) \in \mathcal{J}^{\mathcal{I}}} C_{3}\left|\mathcal{P}_{(a, h)}\right|\left|\mathcal{Q}_{\left(a^{\mathcal{I}}, h^{\mathcal{I}}\right)}\right| A_{3} B_{3} .
$$

Finally, it turns out that $\left|\mathcal{P}_{(a, h)}\right| \leq(u L)^{8 r}$ and $\left|\mathcal{Q}_{\left(a^{\mathcal{I}}, h^{\mathcal{I}}\right)}\right| \leq(u L)^{8 r}$. Therefore, since $r \leq m,(5.42)$ and (5.51) allow us to write, for $(L, \Theta, u, j) \in G_{M}^{m}$ and $L \geq L_{\varepsilon / 2 m+1}$,

$$
Z_{2}(L, \Theta, u, j) \leq(m L)^{16 m} Z_{3}(L, \Theta, u, j) .
$$

The latter is sufficient to conclude that $\psi_{2} \prec \psi_{3}$.

5.2.3. Step 3. For every $(L, \Theta, u, j) \in G_{M}^{m}$ we have, by the definition in (4.45) of $\mathcal{L}\left(l_{A, \Theta, j}, l_{B, \Theta, j} ; u\right)$, that $(a, h) \in \mathcal{J}_{\Theta, L, j}$ and $\left(a^{\mathcal{I}}, h^{\mathcal{I}}\right) \in \mathcal{J}^{\mathcal{I}}$ satisfying $a_{A}+a_{B}+a^{\mathcal{I}}=$ $u L$ and $h_{A}+h_{B}+h^{\mathcal{I}}=L$ also satisfy

$$
\left(\left(\frac{a_{A}}{L}, \frac{a_{B}}{L}, \frac{a^{\mathcal{I}}}{L}\right),\left(\frac{h_{A}}{L}, \frac{h_{B}}{L}, \frac{h^{\mathcal{I}}}{L}\right)\right) \in \mathcal{L}\left(l_{A, \Theta, j}, l_{B, \Theta, j} ; u\right) .
$$

Hence, (5.53) and the definition of $\psi_{\mathcal{I}}$ in (4.48) ensure that, for this choice of $(a, h)$ and $\left(a^{\mathcal{I}}, h^{\mathcal{I}}\right)$,

$$
A_{3} B_{3} \leq e^{u L \psi_{\mathcal{I}}\left(u, l_{A, \Theta, j}, l_{B, \Theta, j}\right)} .
$$

Because of $C_{3}$, the summation in (5.42) is restricted to those $(a, h) \in \mathcal{J}_{\Theta, L, j}$ and $\left(a^{\mathcal{I}}, h^{\mathcal{I}}\right) \in \mathcal{J}^{\mathcal{I}}$ for which $a_{A}, a_{B}, a^{\mathcal{I}} \leq u L$ and $h_{A}, h_{B}, h^{\mathcal{I}} \leq L$. Hence, the summation is restricted to a set of cardinality at most $(u L)^{3} L^{3}$. Consequently, for all $(L, \Theta, u, j) \in G_{M}^{m}$ we have

$$
Z_{3}(L, \Theta, u, j)=\sum_{(a, h) \in \mathcal{J}_{\Theta, L, j}} \sum_{\left(a^{\mathcal{I}}, h^{\mathcal{I}}\right) \in \mathcal{J}^{\mathcal{I}}} A_{4} B_{4} C_{4} \leq(m L)^{3} L^{3} e^{u L \psi_{\mathcal{I}}\left(u, l_{A, \Theta, j}, l_{B, \Theta, j}\right)} .
$$

The latter implies that $\psi_{3} \prec \psi_{4}$ since $\psi_{4}=\psi_{\mathcal{I}}\left(u, l_{A, \Theta, j}, l_{B, \Theta, j}\right)$ by definition (recall (5.13) and (5.26)).

\section{Column-based variational formula}

To derive the slope-based variational formula that is the cornerstone of our analysis, we state and prove in this section an auxiliary variational formula for the quenched free energy per step that involves the fraction of the time spent by the copolymer in each type of block columns and the free energy per step of the copolymer in a given block column. This auxiliary variational formula will be used in Section 7 in combination with Proposition 4.6 to complete the proof of the slope-based variational formula.

With each $\Theta \in \overline{\mathcal{V}}_{M}$ we associate a quantity $u_{\Theta} \in\left[t_{\Theta}, \infty\right)$ indicating how many steps on scale $L_{n}$ the copolymer makes in columns of type $\Theta$, where $t_{\Theta}$ is the 
minimal number of steps required to cross a column of type $\Theta$. These numbers are gathered into the set

$$
\mathcal{B}_{\overline{\mathcal{V}}_{M}}=\left\{\left(u_{\Theta}\right)_{\Theta \in \overline{\mathcal{V}}_{M}} \in \mathbb{R}^{\overline{\mathcal{V}}_{M}}: u_{\Theta} \geq t_{\Theta} \forall \Theta \in \overline{\mathcal{V}}_{M}, \Theta \mapsto u_{\Theta} \text { continuous }\right\},
$$

where the continuity in $\Theta$ is with respect to the distance $d_{M}$ defined in (C.7) in Appendix C.2. We recall Proposition 4.6, which identifies the free energy per step $\psi\left(\Theta, u_{\Theta} ; \alpha, \beta\right)$ associated with the copolymer when crossing a column of type $\Theta$ in $u_{\Theta}$ steps, and we recall that the set $\mathcal{R}_{p, M}$ introduced in Section 4.5.2 gathers the frequencies with which different types of columns can be visited by the copolymer.

Theorem 6.1. (column-based variational formula) For every $(\alpha, \beta) \in \mathrm{CONE}$, and $p \in(0,1)$ the free energy in $(2.9)$ exists for $\mathbb{P}$-a.e. $(\omega, \Omega)$ and in $L^{1}(\mathbb{P})$, and is given by

$$
f(\alpha, \beta ; p)=\sup _{M \geq 1} \sup _{\rho \in \mathcal{R}_{p, M}} \sup _{\left(u_{\Theta}\right)_{\Theta \in \overline{\mathcal{V}}_{M}} \in \mathcal{B}_{\overline{\mathcal{V}}_{M}}} \frac{N(\rho, u)}{D(\rho, u)}
$$

where

$$
\begin{aligned}
& N(\rho, u)=\int_{\overline{\mathcal{V}}_{M}} u_{\Theta} \psi\left(\Theta, u_{\Theta} ; \alpha, \beta\right) \rho(d \Theta), \\
& D(\rho, u)=\int_{\overline{\mathcal{V}}_{M}} u_{\Theta} \rho(d \Theta),
\end{aligned}
$$

with the convention that $N(\rho, u) / D(\rho, u)=-\infty$ when $D(\rho, u)=\infty$.

The present section is technically involved because it goes through a sequence of approximation steps in which the self-averaging of the free energy with respect to $\omega$ and $\Omega$ in the limit as $n \rightarrow \infty$ is proven, and the various ingredients of the variational formula in Theorem 6.1 that were constructed in Section 4 are put together.

In Section 6.1 we introduce additional notation and state Propositions 6.2, 6.3, 6.4 and 6.5 from which Theorem 6.1 is a straightforward consequence. Proposition 6.2 , which deals with $(M, m) \in \mathrm{EIGH}$, is proven in Section 6.2 and the details of the proof are worked out in Sections 6.2.1-6.2.7, organized into 5 Steps that link intermediate free energies. We pass to the limit $m \rightarrow \infty$ with Propositions 6.3 and 6.4 which are proven in Section 6.3 and 6.4, respectively. Finally, we pass to the limit $M \rightarrow \infty$ with Proposition 6.5 which is proven in Section 6.5.

\subsection{Proof of Theorem 6.1.}

6.1.1. Additional notation. Pick $(M, m) \in \mathrm{EIGH}$ and recall that $\Omega$ and $\omega$ are independent, i.e., $\mathbb{P}=\mathbb{P}_{\omega} \times \mathbb{P}_{\Omega}$. For $\Omega \in\{A, B\}^{\mathbb{N}_{0} \times \mathbb{Z}}, \omega \in\{A, B\}^{\mathbb{N}}, n \in \mathbb{N}$ and $(\alpha, \beta) \in \mathrm{CONE}$, define

$$
f_{1, n}^{\omega, \Omega}(M, m ; \alpha, \beta)=\frac{1}{n} \log Z_{1, n, L_{n}}^{\omega, \Omega}(M, m) \quad \text { with } \quad Z_{1, n, L_{n}}^{\omega, \Omega}(M, m)=\sum_{\pi \in \mathcal{W}_{n, M}^{m},} e^{H_{n, L_{n}}^{\omega, \Omega}(\pi)}
$$

where $\mathcal{W}_{n, M}^{m}$ contains those paths in $\mathcal{W}_{n, M}$ that, in each column, make at most $m L_{n}$ steps. We also restrict the set $\mathcal{R}_{p, M}$ in (4.7) to those limiting empirical measures whose support is included in $\overline{\mathcal{V}}_{M}^{m}$, i.e., those measures charging the types of column 
that can be crossed in less than $m L_{n}$ steps only. To that aim we recall (4.57) and define, for $\Omega \in\{A, B\}^{\mathbb{N}_{0} \times \mathbb{Z}}$ and $N \in \mathbb{N}$,

$$
\begin{aligned}
\mathcal{R}_{M, N}^{\Omega, m}=\{ & \rho_{N}(\Omega, \Pi, b, x) \text { with } b=\left(b_{j}\right)_{j \in \mathbb{N}_{0}} \in\left(\mathbb{Q}_{(0,1]}\right)^{\mathbb{N}_{0}}, \\
& \Pi=\left(\Pi_{j}\right)_{j \in \mathbb{N}_{0}} \in\{0\} \times \mathbb{Z}^{\mathbb{N}}:\left|\Delta \Pi_{j}\right| \leq M \quad \forall j \in \mathbb{N}_{0}, \\
& \left.x=\left(x_{j}\right)_{j \in \mathbb{N}_{0}} \in\{1,2\}^{N_{0}}:\left(\Omega\left(j, \Pi_{j}+\cdot\right), \Delta \Pi_{j}, b_{j}, b_{j+1}, x_{j}\right) \in \mathcal{V}_{M}^{m}\right\}
\end{aligned}
$$

which is a subset of $\mathcal{R}_{M, N}^{\Omega}$ and allows us to define

$$
\mathcal{R}_{M}^{\Omega, m}=\operatorname{closure}\left(\cap_{N^{\prime} \in \mathbb{N}} \cup_{N \geq N^{\prime}} \mathcal{R}_{M, N}^{\Omega, m}\right),
$$

which, for $\mathbb{P}$-a.e. $\Omega$ is equal to $\mathcal{R}_{p, M}^{m} \subsetneq \mathcal{R}_{p, M}$.

At this stage, we further define,

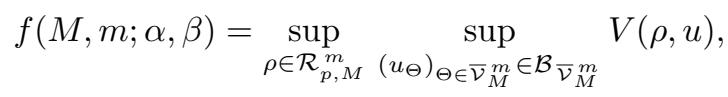

where

$$
V(\rho, u)=\frac{\int_{\overline{\mathcal{V}}_{M}^{m}} u_{\Theta} \psi\left(\Theta, u_{\Theta} ; \alpha, \beta\right) \rho(d \Theta)}{\int_{\overline{\mathcal{V}}_{M}^{m}}^{m} u_{\Theta} \rho(d \Theta)},
$$

where (recall (4.28))

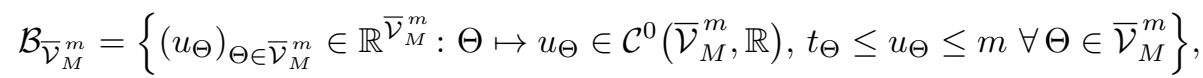

and where $\overline{\mathcal{V}}_{M}^{m}$ is endowed with the distance $d_{M}$ defined in (C.7) in Appendix C.2.

Let $\mathcal{W}_{n, M}^{*, m} \subset \mathcal{W}_{n, M}^{m}$ be the subset consisting of those paths whose endpoint lies at the boundary between two columns of blocks, i.e., satisfies $\pi_{n, 1} \in \mathbb{N} L_{n}$. Recall (6.4), and define $Z_{n, L_{n}}^{*, \omega, \Omega}(M)$ and $f_{1, n}^{*, \omega, \Omega}(M, m ; \alpha, \beta)$ as the counterparts of $Z_{n, L_{n}}^{\omega, \Omega}(M, m)$ and $f_{1, n}^{\omega, \Omega}(M, m ; \alpha, \beta)$ when $\mathcal{W}_{n, M}^{m}$ is replaced by $\mathcal{W}_{n, M}^{*, m}$. Then there exists a constant $c>0$, depending on $\alpha$ and $\beta$ only, such that

$$
\begin{aligned}
& Z_{1, n, L_{n}}^{\omega, \Omega}(M, m) e^{-c L_{n}} \leq Z_{1, n, L_{n}}^{*, \omega, \Omega}(M, m) \leq Z_{1, n, L_{n}}^{\omega, \Omega}(M, m), \\
& n \in \mathbb{N}, \omega \in\{A, B\}^{\mathbb{N}}, \Omega \in\{A, B\}^{\mathbb{N}_{0} \times \mathbb{Z}} .
\end{aligned}
$$

The left-hand side of the latter inequality is obtained by changing the last $L_{n}$ steps of each trajectory in $\mathcal{W}_{n, M}^{m}$ to make sure that the endpoint falls in $L_{n} \mathbb{N}$. The energetic and entropic cost of this change are obviously $O\left(L_{n}\right)$. By assumption, $\lim _{n \rightarrow \infty} L_{n} / n=0$, which together with (6.10) implies that the limits of $f_{1, n}^{\omega, \Omega}(M, m ; \alpha, \beta)$ and $f_{1, n}^{*, \omega, \Omega}(M, m ; \alpha, \beta)$ as $n \rightarrow \infty$ are the same. In the sequel we will therefore restrict the summation in the partition function to $\mathcal{W}_{n, M}^{*, m}$ and drop the $*$ from the notations.

Finally, let

$$
\begin{aligned}
& f_{1, n}^{\Omega}(M, m ; \alpha, \beta)=\mathbb{E}_{\omega}\left[f_{1, n}^{\omega, \Omega}(M, m ; \alpha, \beta)\right], \\
& f_{1, n}(M, m ; \alpha, \beta)=\mathbb{E}_{\omega, \Omega}\left[f_{1, n}^{\omega, \Omega}(M, m ; \alpha, \beta)\right],
\end{aligned}
$$

and recall (2.8) and (4.11) to set

$$
f_{n}^{\Omega}(\alpha, \beta)=\mathbb{E}_{\omega}\left[f_{n}^{\omega, \Omega}(\alpha, \beta)\right], \quad f_{n}^{\Omega}(M ; \alpha, \beta)=\mathbb{E}_{\omega}\left[f_{n}^{\omega, \Omega}(M ; \alpha, \beta)\right] .
$$


6.1.2. Key Propositions. Theorem 6.1 is a consequence of Propositions 6.3, 6.4 and 6.5 stated below and proven in Sections 6.3.1-6.3.3, Section 6.4 and Sections 6.5.16.5.7, respectively.

Proposition 6.2, that is stated first is required to prove Proposition 6.3 and will be proven in Sections 6.2.1-6.2.5.

Proposition 6.2. For all $(M, m) \in \mathrm{EIGH}$,

$$
\lim _{n \rightarrow \infty} f_{1, n}^{\Omega}(M, m ; \alpha, \beta)=f(M, m ; \alpha, \beta) \quad \text { for } \mathbb{P}-\text { a.e. } \Omega \text {. }
$$

Proposition 6.3. For all $M \in \mathbb{N}$,

$$
\lim _{n \rightarrow \infty} f_{n}^{\Omega}(M ; \alpha, \beta)=\sup _{m \geq M+2} f(M, m ; \alpha, \beta) \quad \text { for } \mathbb{P}-\text { a.e. } \Omega .
$$

Proposition 6.4. For all $M \in \mathbb{N}$,

$$
\sup _{m \geq M+2} f(M, m ; \alpha, \beta)=\sup _{\rho \in \mathcal{R}_{p, M}} \sup _{\left(u_{\Theta}\right)_{\Theta \in \overline{\mathcal{V}}_{M}} \in \mathcal{B}_{\overline{\mathcal{V}}_{M}}} V(\rho, u) .
$$

In the righthand side of (6.15), we recognize the variational formula of Theorem 6.1 and with $\mathcal{B}_{\overline{\mathcal{V}}_{M}}$ defined in (4.18).

\section{Proposition 6.5.}

$$
\limsup _{n \rightarrow \infty} f_{n}^{\Omega}(\alpha, \beta) \leq \sup _{M \geq 1} \lim _{n \rightarrow \infty} f_{n}^{\Omega}(M ; \alpha, \beta) \quad \text { for } \mathbb{P}-\text { a.e. } \Omega .
$$

Proof of Theorem 6.1 subject to Propositions 6.3, 6.4 and 6.5. With Propositions 6.3, 6.4 and 6.5 in hand, the proof of Theorem 6.1 will be complete once we show that

$$
\lim _{n \rightarrow \infty}\left|f_{n}^{\omega, \Omega}(\alpha, \beta)-f_{n}^{\Omega}(\alpha, \beta)\right|=0 \quad \text { for } \mathbb{P}-\text { a.e. }(\omega, \Omega) .
$$

To that aim, we note that for all $n \in \mathbb{N}$ the $\Omega$-dependence of $f_{n}^{\omega, \Omega}(\alpha, \beta)$ is restricted to $\left\{\Omega_{x}: x \in G_{n}\right\}$ with $G_{n}=\left\{0, \ldots, \frac{n}{L_{n}}\right\} \times\left\{-\frac{n}{L_{n}}, \ldots, \frac{n}{L_{n}}\right\}$. Thus, for $n \in \mathbb{N}$ and $\varepsilon>0$ we set

$$
\left.A_{\varepsilon, n}=\left\{\left|f_{n}^{\omega, \Omega}(\alpha, \beta)-f_{n}^{\Omega}(\alpha, \beta)\right|>\varepsilon\right)\right\},
$$

and by independence of $\omega$ and $\Omega$ we can write

$$
\begin{aligned}
\mathbb{P}_{\omega, \Omega}\left(A_{\varepsilon, n}\right) & =\sum_{\Upsilon \in\{A, B\}^{G_{n}}} \mathbb{P}_{\omega, \Omega}\left(A_{\varepsilon, n} \cap\left\{\Omega_{G_{n}}=\Upsilon\right\}\right) \\
& =\sum_{\Upsilon \in\{A, B\}^{G_{n}}} \mathbb{P}_{\omega}\left(\left|f_{n}^{\omega, \Upsilon}(\alpha, \beta)-f_{n}^{\Upsilon}(\alpha, \beta)\right|>\varepsilon\right) \mathbb{P}_{\Omega}\left(\left\{\Omega_{G_{n}}=\Upsilon\right\}\right) .
\end{aligned}
$$

At this stage, for each $n \in \mathbb{N}$ we can apply the concentration inequality (D.3) in Appendix D with $\Gamma=\mathcal{W}_{n}, l=n, \eta=\varepsilon n$,

$$
\xi_{i}=-\alpha 1\left\{\omega_{i}=A\right\}+\beta 1\left\{\omega_{i}=B\right\}, \quad i \in \mathbb{N},
$$

and with $T(x, y)$ indicating in which block step $(x, y)$ lies in. Therefore, there exist $C_{1}, C_{2}>0$ such that for all $n \in \mathbb{N}$ and all $\Upsilon \in\{A, B\}^{G_{n}}$ we have

$$
\mathbb{P}_{\omega}\left(\left|f_{n}^{\omega, \Upsilon}(M, m ; \alpha, \beta)-f_{n}^{\Upsilon}(M, m ; \alpha, \beta)\right|>\varepsilon\right) \leq C_{1} e^{-C_{2} \varepsilon^{2} n},
$$

which, together with (6.19) yields $\mathbb{P}_{\omega, \Omega}\left(A_{\varepsilon, n}\right) \leq C_{1} e^{-C_{2} \varepsilon^{2} n}$ for all $n \in \mathbb{N}$. By using the Borel-Cantelli Lemma, we obtain (6.17). 
6.2. Proof of Proposition 6.2. Pick $(M, m) \in$ EIGH and $(\alpha, \beta) \in$ CONE. In Steps 12 in Sections 6.2.1-6.2.2 we introduce an intermediate free energy $f_{3, n}^{\Omega}(M, m ; \alpha, \beta)$ and show that

$$
\lim _{n \rightarrow \infty}\left|f_{1, n}^{\Omega}(M, m ; \alpha, \beta)-f_{3, n}^{\Omega}(M, m ; \alpha, \beta)\right|=0 \quad \forall \Omega \in\{A, B\}^{\mathbb{N}_{0} \times \mathbb{Z}} .
$$

Next, in Steps 3-4 in Sections 6.2.3-6.2.4 we show that

$$
\limsup _{n \rightarrow \infty} f_{3, n}^{\Omega}(M, m ; \alpha, \beta)=f(M, m ; \alpha, \beta) \quad \text { for } \mathbb{P}-\text { a.e. } \Omega,
$$

while in Step 5 in Section 6.2.5 we prove that

$$
\liminf _{n \rightarrow \infty} f_{3, n}^{\Omega}(M, m ; \alpha, \beta)=\limsup _{n \rightarrow \infty} f_{3, n}^{\Omega}(M, m ; \alpha, \beta) \quad \text { for } \mathbb{P}-\text { a.e. } \Omega .
$$

Combing (6.22-6.24) we get

$$
\liminf _{n \rightarrow \infty} f_{1, n}^{\Omega}(M, m ; \alpha, \beta)=\limsup _{n \rightarrow \infty} f_{1, n}^{\Omega}(M, m ; \alpha, \beta)=f(M, m ; \alpha, \beta) \text { for } \mathbb{P}-\text { a.e. } \Omega \text {, }
$$

which completes the proof of Proposition 6.2.

In the proof we need the following order relation.

Definition 6.6. For $g, \widetilde{g}: \mathbb{N}^{3} \times \operatorname{CONE} \mapsto \mathbb{R}$, write $g \prec \widetilde{g}$ if for all $(M, m) \in$ EIGH, $(\alpha, \beta) \in \mathrm{CONE}$ and $\varepsilon>0$ there exists an $n_{\varepsilon} \in \mathbb{N}$ such that

$$
g(n, M, m ; \alpha, \beta) \leq \widetilde{g}(n, M, m ; \alpha, \beta)+\varepsilon \quad \forall n \geq n_{\varepsilon} .
$$

The proof of (6.22) will be complete once we show that $f_{1}^{\Omega} \prec f_{3}^{\Omega}$ and $f_{3}^{\Omega} \prec f_{1}^{\Omega}$ for all $\Omega \in\{A, B\}^{\mathbb{N}_{0} \times \mathbb{Z}}$. We will focus on $f_{1}^{\Omega} \prec f_{3}^{\Omega}$, since the proof of the latter can be easily adapted to obtain $f_{3}^{\Omega} \prec f_{1}^{\Omega}$. To prove $f_{1}^{\Omega} \prec f_{3}^{\Omega}$ we introduce another intermediate free energy $f_{2}^{\Omega}$, and we show that $f_{1}^{\Omega} \prec f_{2}^{\Omega}$ and $f_{2}^{\Omega} \prec f_{3}^{\Omega}$.

For $L \in \mathbb{N}$, let

$$
\mathcal{D}_{L}^{M}=\left\{\Xi=\left(\Delta \Pi, b_{0}, b_{1}\right) \in\{-M, \ldots, M\} \times\left\{\frac{1}{L}, \frac{2}{L}, \ldots, 1\right\}^{2}\right\} .
$$

For $L, N \in \mathbb{N}$, let

$$
\begin{aligned}
\widetilde{\mathcal{D}}_{L, N}^{M}=\left\{\Theta_{\text {traj }}=\left(\Xi_{i}\right)_{i \in\{0, \ldots, N-1\}}\right. & \in\left(\mathcal{D}_{L}^{M}\right)^{N}: \\
& \left.b_{0,0}=\frac{1}{L}, b_{0, i}=b_{1, i-1} \forall 1 \leq i \leq N-1\right\},
\end{aligned}
$$

and with each $\Theta_{\text {traj }} \in \widetilde{\mathcal{D}}_{L, N}^{M}$ associate the sequence $\left(\Pi_{i}\right)_{i=0}^{N}$ defined by $\Pi_{0}=0$ and $\Pi_{i}=\sum_{j=0}^{i-1} \Delta \Pi_{j}$ for $1 \leq i \leq N$. Next, for $\Omega \in\{A, B\}^{\mathbb{N}_{0} \times \mathbb{Z}}$ and $\Theta_{\text {traj }} \in \widetilde{\mathcal{D}}_{L, N}^{M}$, set

$$
\mathcal{X}_{\Theta_{\mathrm{traj}}, \Omega}^{M, m}=\left\{x \in\{1,2\}^{\{0, \ldots, N-1\}}:\left(\Omega\left(i, \Pi_{i}+\cdot\right), \Xi_{i}, x_{i}\right) \in \mathcal{V}_{M}^{m} \forall 0 \leq i \leq N-1\right\},
$$

and, for $x \in \mathcal{X}_{\Theta_{\text {traj }}^{M, m}, \Omega}$, set

$$
\Theta_{i}=\left(\Omega\left(i, \Pi_{i}+\cdot\right), \Xi_{i}, x_{i}\right) \text { for } i \in\{0, \ldots, N-1\}
$$

and

$$
\begin{aligned}
\mathcal{U}_{\Theta_{\text {traj }}, x, n}^{M, m, L}=\{u= & \left(u_{i}\right)_{i \in\{0, \ldots, N-1\}} \in[1, m]^{N}: \\
& \left.u_{i} \in t_{\Theta_{i}}+\frac{2 \mathbb{N}}{L} \quad \forall 0 \leq i \leq N-1, \sum_{i=0}^{N-1} u_{i}=\frac{n}{L}\right\} .
\end{aligned}
$$

Note that $\mathcal{U}_{\Theta_{\text {traj }}, x, n}^{M, m, L}$ is empty when $N \notin\left[\frac{n}{m L}, \frac{n}{L}\right]$. 
For $\pi \in \mathcal{W}_{n, M}^{m}$, we let $N_{\pi}$ be the number of columns crossed by $\pi$ after $n$ steps. We denote by $\left(u_{0}(\pi), \ldots, u_{N_{\pi}-1}(\pi)\right)$ the time spent by $\pi$ in each column divided by $L_{n}$, and we set $\widetilde{u}_{0}(\pi)=0$ and $\widetilde{u}_{j}(\pi)=\sum_{k=0}^{j-1} u_{k}(\pi)$ for $1 \leq j \leq N_{\pi}$. With these notations, the partition function in $(6.4)$ can be rewritten as

$$
Z_{1, n, L_{n}}^{\omega, \Omega}(M, m)=\sum_{N=n / m L_{n}}^{n / L_{n}} \sum_{\Theta_{\text {traj }} \in \widetilde{\mathcal{D}}_{L_{n}, N}^{M}} \sum_{x \in \mathcal{X}_{\Theta_{\text {traj }}^{M, m}}} \sum_{u \in \mathcal{U}_{\Theta_{\text {traj }}^{M, m, n}}} A_{1},
$$

with (recall $(4.34))$

$$
A_{1}=\prod_{i=0}^{N-1} Z_{L_{n}}^{\theta^{\tilde{u}_{i} L_{n}}(\omega)}\left(\Omega\left(i, \Pi_{i}+\cdot\right), \Xi_{i}, x_{i}, u_{i}\right) .
$$

6.2.1. Step 1. In this step we average over the disorder $\omega$ in each column. To that end, we set

$$
f_{2, n}^{\Omega}(M, m ; \alpha, \beta)=\frac{1}{n} \log Z_{2, n, L_{n}}^{\Omega}(M, m)
$$

with

$$
Z_{2, n, L_{n}}^{\Omega}(M, m)=\sum_{N=n / m L_{n}}^{n / L_{n}} \sum_{\Theta_{\text {traj }} \in \widetilde{\mathcal{D}}_{L_{n}, N}^{M}} \sum_{x \in \mathcal{X}_{\Theta_{\text {traj }}^{M, m}}} \sum_{u \in \mathcal{U}_{\Theta_{\text {traj }}^{M, m, n}, L_{n}}} A_{2}
$$

where

$$
A_{2}=\prod_{i=0}^{N-1} e^{\mathbb{E}_{\omega}\left[\log Z_{L_{n}}^{\theta^{\tilde{u}_{i}(\omega)}}\left(\Omega\left(i, \Pi_{i}+\cdot\right), \Xi_{i}, x_{i}, u_{i}\right)\right]}=\prod_{i=0}^{N-1} e^{u_{i} L_{n} \psi_{L_{n}}\left(\Omega\left(i, \Pi_{i}+\cdot\right), \Xi_{i}, x_{i}, u_{i}\right)} .
$$

Note that the $\omega$-dependence has been removed from $Z_{2, n, L_{n}}^{\Omega}(M, m)$.

To prove that $f_{1}^{\Omega} \prec f_{2}^{\Omega}$, we need to show that for all $\varepsilon>0$ there exists an $n_{\varepsilon} \in \mathbb{N}$ such that, for $n \geq n_{\varepsilon}$ and all $\Omega$,

$$
\mathbb{E}_{\omega}\left[\log Z_{1, n, L_{n}}^{\omega, \Omega}(M, m)\right] \leq \log Z_{2, n, L_{n}}^{\Omega}(M, m)+\varepsilon n .
$$

To this end, we rewrite $Z_{1, n, L_{n}}^{\omega, \Omega}(M, m)$ as

$$
Z_{1, n, L_{n}}^{\omega, \Omega}(M, m)=\sum_{N=n / m L_{n}}^{n / L_{n}} \sum_{\Theta_{\text {traj }} \in \widetilde{\mathcal{D}}_{L_{n}, N}^{M}} \sum_{x \in \mathcal{X}_{\Theta_{\text {traj }}^{M, \Omega}}} \sum_{u \in \mathcal{U}_{\Theta_{\text {traj }}^{M, x, n}}} A_{2} \frac{A_{1}}{A_{2}},
$$

where we note that

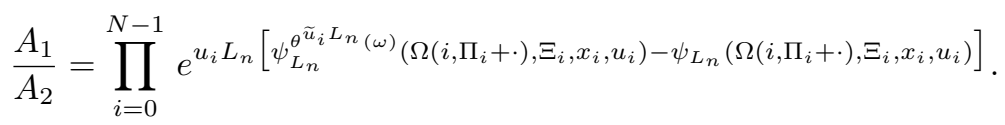

In order to average over $\omega$, we apply a concentration of measure inequality. Set

$$
\mathcal{K}_{n}=\bigcup_{N=n / m L_{n}}^{n / L_{n}} \bigcup_{\Theta_{\text {traj }} \in \widetilde{\mathcal{D}}_{L_{n}, N}^{M}} \bigcup_{x \in \mathcal{X}_{\Theta_{\text {traj }}^{M, m}}} \bigcup_{u \in \mathcal{U}_{\Theta_{\text {traj }}^{M, m, n}, n}}\left\{\left|\log A_{1}-\log A_{2}\right| \geq \varepsilon n\right\},
$$


and note that $\omega \in \mathcal{K}_{n}^{c}$ implies that $Z_{1, n, L_{n}}^{\omega, \Omega}(M, m) \leq e^{\varepsilon n} Z_{2, n, L_{n}}^{\Omega}(M, m)$. Consequently, we can write

$$
\begin{aligned}
\mathbb{E}_{\omega}\left[\log Z_{1, n, L_{n}}^{\omega, \Omega}(M, m)\right] & =\mathbb{E}_{\omega}\left[\log Z_{1, n, L_{n}}^{\omega, \Omega}(M, m) 1_{\left\{\mathcal{K}_{n}\right\}}\right]+\mathbb{E}_{\omega}\left[\log Z_{1, n, L_{n}}^{\omega, \Omega}(M, m) 1_{\left\{\mathcal{K}_{n}^{c}\right\}}\right] \\
& \leq \mathbb{E}_{\omega}\left[\log Z_{1, n, L_{n}}^{\omega, \Omega}(M, m) 1_{\left\{\mathcal{K}_{n}\right\}}\right]+\log Z_{2, n, L_{n}}^{\Omega}(M, m)+\varepsilon n .
\end{aligned}
$$

We can now use the uniform bound in (4.37) to control the first term in the righthand side of (6.41), to obtain

$$
\mathbb{E}_{\omega}\left[\log Z_{1, n, L_{n}}^{\omega, \Omega}(M, m)\right] \leq \log Z_{2, n, L_{n}}^{\Omega}(M, m)+\varepsilon n+C_{\mathrm{uf}}(\alpha) n \mathbb{P}_{\omega}\left(\mathcal{K}_{n}\right) .
$$

Therefore the proof of this step will be complete once we show that $\mathbb{P}_{\omega}\left(\mathcal{K}_{n}\right)$ vanishes as $n \rightarrow \infty$.

Lemma 6.7. There exist $C_{1}, C_{2}>0$ such that, for all $\varepsilon>0, n \in \mathbb{N}, N \in$ $\left\{\frac{n}{m L_{n}}, \ldots, \frac{n}{L_{n}}\right\}, \Omega \in\{A, B\}^{\mathbb{N}_{0} \times \mathbb{Z}}, \Theta_{\text {traj }} \in \widetilde{\mathcal{D}}_{L_{n}, N}^{M}, x \in \mathcal{X}_{\Theta_{\text {traj }}, \Omega}^{M, m}$ and $u \in \mathcal{U}_{\Theta_{\text {traj }}, x, n}^{M, m, L_{n}}$,

$$
\mathbb{P}_{\omega}\left(\left|\log A_{1}-\log A_{2}\right| \geq \varepsilon n\right) \leq C_{1} e^{-C_{2} \varepsilon^{2} n} .
$$

Proof: Pick $\Theta_{\text {traj }} \in \widetilde{\mathcal{D}}_{L_{n}, N}^{M}, x \in \mathcal{X}_{\Theta_{\text {traj }, \Omega}}^{M, m}$ and $u \in \mathcal{U}_{\Theta_{\text {traj }}, x, n}^{M, m, L_{n}}$, and consider the subset $\Gamma$ of $\mathcal{W}_{n, M}^{m}$ consisting of those paths of length $n$ that first cross the $\left(\Omega(0, \cdot), \Xi_{0}, x_{0}\right)$ column such that $\pi_{0}=(0,1)$ and $\pi_{\widetilde{u}_{1} L_{n}}=\left(1, \Pi_{1}+b_{1,0}\right) L_{n}$, then cross the $\left(\Omega(1, \cdot), \Xi_{1}, x_{1}\right)$ column such that $\pi_{\widetilde{u}_{1} L_{n}+1}=\left(1+1 / L_{n}, \Pi_{1}+b_{1,0}\right) L_{n}$ and $\pi_{\widetilde{u}_{2} L_{n}}=$ $\left(2, \Pi_{2}+b_{1,1}\right) L_{n}$, and so on. We can apply the concentration of measure inequality stated in (D.3) to the set $\Gamma$ defined above, with $l=n, \eta=\varepsilon n$,

$$
\xi_{i}=-\alpha 1\left\{\omega_{i}=A\right\}+\beta 1\left\{\omega_{i}=B\right\}, \quad i \in \mathbb{N},
$$

and with $T(x, y)$ indicating in which block step $(x, y)$ lies in. After noting that $\mathbb{E}_{\omega}\left(\log A_{1}\right)=\log A_{2}$, we obtain that there exist $C_{1}, C_{2}>0$ such that, for all $n \in \mathbb{N}, N \in\left\{\frac{n}{m L_{n}}, \ldots, \frac{n}{L_{n}}\right\}, \Omega \in\{A, B\}^{\mathbb{N}_{0} \times \mathbb{Z}}, \Theta_{\text {traj }} \in \widetilde{\mathcal{D}}_{L_{n}, N}^{M}, x \in \mathcal{X}_{\Theta_{\text {traj }, \Omega}^{M}}^{M, m}$ and $u \in \mathcal{U}_{\Theta_{\text {traj }}, x, n}^{M, m, L_{n}}$,

$$
\mathbb{P}\left(\left|\log A_{1}-\log A_{2}\right| \geq \varepsilon n\right) \leq C_{1} e^{-C_{2} \varepsilon^{3} n} .
$$

It now suffices to remark that

$$
\left|\left\{\left(N, \Theta_{\text {traj }}, x, u\right): N \in\left\{\frac{n}{m L_{n}}, \ldots, \frac{n}{L_{n}}\right\}, \Theta_{\text {traj }} \in \widetilde{\mathcal{D}}_{L_{n}, N}^{M}, x \in \mathcal{X}_{\Theta_{\text {traj }, \Omega}}^{M, m}, u \in \mathcal{U}_{\Theta_{\text {traj }}, x, n}^{M, m, L_{n}}\right\}\right|
$$

grows subexponentially in $n$ to obtain that $f_{1}^{\Omega} \prec f_{2}^{\Omega}$ for all $\Omega$.

6.2.2. Step 2. In this step we replace the finite-size free energy $\psi_{L_{n}}$ by its limit $\psi$. To do so we introduce a third intermediate free energy,

$$
f_{3, n}^{\Omega}(M, m ; \alpha, \beta)=\mathbb{E}\left[\frac{1}{n} \log Z_{3, n, L_{n}}^{\Omega}(M, m)\right],
$$

where

$$
Z_{3, n, L_{n}}^{\Omega}(M, m)=\sum_{N=n / m L_{n}}^{n / L_{n}} \sum_{\Theta_{\text {traj }} \in \widetilde{\mathcal{D}}_{L_{n}, N}^{M}} \sum_{x \in \mathcal{X}_{\Theta_{\text {traj }}^{M, m}}} \sum_{u \in \mathcal{U}_{\Theta_{\text {traj }, x, n}^{M, m, L_{n}}}} A_{3}
$$


with

$$
A_{3}=\prod_{i=0}^{N-1} e^{u_{i} L_{n} \psi\left(\Omega\left(i, \Pi_{i}+\cdot\right), \Xi_{i}, x_{i}, u_{i}\right)}
$$

For all $\Omega$,

$$
\frac{A_{2}}{A_{3}}=\prod_{i=0}^{N-1} e^{u_{i} L_{n}\left[\psi_{L_{n}}\left(\Omega\left(i, \Pi_{i}+\cdot\right), \Xi_{i}, x_{i}, u_{i}\right)-\psi\left(\Omega\left(i, \Pi_{i}+\cdot\right), \Xi_{i}, x_{i}, u_{i}\right)\right]},
$$

and, for all $i \in\{0, \ldots, N-1\}$, we have $\left(\Omega\left(i, \Pi_{i}+\cdot\right), \Xi_{i}, x_{i}, u_{i}\right) \in \mathcal{V}_{M}^{*, m}$, so that Proposition 4.5 can be applied.

6.2.3. Step 3. In this step we want the variational formula (6.7) to appear. Recall (4.56) and define, for $n \in \mathbb{N},(M, m) \in$ EIGH, $N \in\left\{\frac{n}{m L_{n}}, \ldots, \frac{n}{L_{n}}\right\}, \Theta_{\text {traj }} \in \widetilde{\mathcal{D}}_{L_{n}, N}^{M}$ and $x \in \mathcal{X}_{\Theta_{\text {traj }}^{M, \Omega}}^{M,}$,

$$
\Theta_{j}=\left(\Omega\left(j, \Pi_{j}+\cdot\right), \Xi_{j}, x_{j}\right), \quad j=0, \ldots, N-1,
$$

and

$$
\rho_{\Theta_{\text {traj }}, x}^{\Omega}\left(\Theta, \Theta^{\prime}\right)=\frac{1}{N} \sum_{j=1}^{N} 1_{\left\{\left(\Theta_{j-1}, \Theta_{j}\right)=\left(\Theta, \Theta^{\prime}\right)\right\}},
$$

and, for $u \in \mathcal{U}_{\Theta_{\text {traj }}, x, n}^{M, L_{n}}$,

$$
H^{\Omega}\left(\Theta_{\text {traj }}, x, u\right)=\sum_{j=0}^{N-1} u_{j} \psi\left(\Theta_{j}, u_{j}\right) .
$$

In terms of these quantities we can rewrite $Z_{3, n, L_{n}}^{\Omega}(M, m)$ in (6.48) as

$$
Z_{3, n, L_{n}}^{\Omega}(M, m)=\sum_{N=n / m L_{n}}^{n / L_{n}} \sum_{\Theta_{\text {traj }} \in \widetilde{\mathcal{D}}_{L_{n}, N}^{M}} \sum_{x \in \mathcal{X}_{\Theta_{\text {traj }}^{M, m}}} \sum_{u \in \mathcal{U}_{\Theta_{\text {traj }}^{M, x, n}, L_{n}}} e^{L_{n} H^{\Omega}\left(\Theta_{\text {traj }}, x, u\right)}
$$

For $n \in \mathbb{N}$, denote by

$$
N_{n}^{\Omega}, \quad \Theta_{\text {traj }, n}^{\Omega} \in \widetilde{\mathcal{D}}_{L_{n}, N_{n}^{\Omega}}^{M}, \quad x_{n}^{\Omega} \in \mathcal{X}_{\Theta_{\mathrm{traj}, n}^{\Omega}, \Omega}^{M, m}, \quad u_{n}^{\Omega} \in \mathcal{U}_{\Theta_{\mathrm{traj}, n}^{\Omega}, x_{n}^{\Omega}, n}^{M, m, L_{n}},
$$

the indices in the summation set of (6.54) that maximize $H^{\Omega}\left(\Theta_{\text {traj }}, x, u\right)$. For ease of notation we put

$$
\Theta_{\mathrm{traj}, n}^{\Omega}=\left(\Xi_{j}^{n}\right)_{j=0}^{N_{n}^{\Omega}-1}, \quad x_{n}^{\Omega}=\left(x_{j}^{n}\right)_{j=0}^{N_{n}^{\Omega}-1}, \quad u_{n}^{\Omega}=\left(u_{j}^{n}\right)_{j=0}^{N_{n}^{\Omega}-1},
$$

and

$$
\begin{aligned}
& c_{n}=\mid\left\{\left(N, \Theta_{\text {traj }}, x, u\right):\right. \\
& \left.\quad \frac{n}{m L_{n}} \leq N \leq \frac{n}{L_{n}}, \Theta_{\text {traj }} \in \widetilde{\mathcal{D}}_{L_{n}, N}^{M}, x \in \mathcal{X}_{\Theta_{\text {traj }}, \Omega}^{M, m}, u \in \mathcal{U}_{\Theta_{\text {traj }}, x, n}^{M, m, L_{n}}\right\} \mid .
\end{aligned}
$$

Then we can estimate

$$
\frac{1}{n} \log Z_{3, n, L_{n}}^{\Omega}(M, m) \leq \frac{1}{n} \log c_{n}+\frac{L_{n}}{n} \sum_{j=0}^{N_{n}^{\Omega}-1} u_{j}^{n} \psi\left(\Theta_{j}^{n}, u_{j}^{n}\right) .
$$


We next note that $u \mapsto u \psi(\Theta, u)$ is concave for all $\Theta \in \overline{\mathcal{V}}_{M}$ (see Lemma C.4). Hence, after setting

$$
v_{\Theta}^{n}=\sum_{j=0}^{N_{n}^{\Omega}-1} 1_{\left\{\Theta_{j}^{n}=\Theta\right\}} u_{j}^{n}, \quad d_{\Theta}^{n}=\sum_{j=0}^{N_{n}^{\Omega}-1} 1_{\left\{\Theta_{j}^{n}=\Theta\right\}}, \quad \Theta \in \overline{\mathcal{V}}_{M}^{m},
$$

we can estimate

$$
\sum_{j=0}^{N_{n}^{\Omega}-1} 1_{\left\{\Theta_{j}^{n}=\Theta\right\}} u_{j}^{n} \psi\left(\Theta_{j}^{n}, u_{j}^{n}\right) \leq v_{\Theta}^{n} \psi\left(\Theta, \frac{v_{\Theta}^{n}}{d_{\Theta}^{n}}\right) \quad \text { for } \quad \Theta \in \overline{\mathcal{V}}_{M}^{m}: d_{\Theta}^{n} \geq 1 .
$$

Next, we recall (6.52) and we set $\rho_{n}=\rho_{\Theta_{\text {traj }, n}^{\Omega}, x_{n}^{\Omega}}^{\Omega}$, so that $\rho_{n, 1}(\Theta)=d_{\Theta}^{n} / N_{n}^{\Omega}$ for all $\Theta \in \overline{\mathcal{V}}_{M}^{m}$. Since $\left\{\Theta \in \overline{\mathcal{V}}_{M}^{m}: d_{\Theta}^{n} \geq 1\right\}$ is a finite subset of $\overline{\mathcal{V}}_{M}^{m}$, we can easily extend $\Theta \mapsto v_{\Theta}^{n} / d_{\Theta}^{n}$ from $\left\{\Theta \in \overline{\mathcal{V}}_{M}: d_{\Theta}^{n} \geq 1\right\}$ to $\overline{\mathcal{V}}_{M}^{m}$ as a continuous function. Moreover, $\sum_{j=0}^{N_{n}^{\Omega}-1} u_{j}^{n}=n / L_{n}$ implies that $N_{n}^{\Omega} \int_{\overline{\mathcal{V}}_{M}^{m}} v_{\Theta}^{n} / d_{\Theta}^{n} \rho_{n, 1}(d \Theta)=n / L_{n}$, which, together with (6.58) and (6.60) gives

$$
\frac{1}{n} \log Z_{3, n, L_{n}}^{\Omega}(M, m) \leq \sup _{u \in \mathcal{B} \overline{\mathcal{V}}_{M}^{m}} \frac{\int_{\overline{\mathcal{V}}_{M}^{m}}^{m} u_{\Theta} \psi\left(\Theta, u_{\Theta}\right) \rho_{n}(d \Theta)}{\int_{\overline{\mathcal{V}}_{M}^{m}}^{m} u_{\Theta} \rho_{n}(d \Theta)}+o(1), \quad n \rightarrow \infty,
$$

where we use that $\lim _{n \rightarrow \infty} \frac{1}{n} \log c_{n}=0$. In what follows, we abbreviate the first term in the right-hand side of the last display by $l_{n}$. We want to show that $\lim \sup _{n \rightarrow \infty} \frac{1}{n} \log Z_{3, n, L_{n}}^{\Omega}(M, m) \leq f(M, m ; \alpha, \beta)$. To that end, we assume that $\frac{1}{n} \log Z_{3, n, L_{n}}^{\Omega}(M, m)$ converges to some $t \in \mathbb{R}$ and we prove that $t \leq f(M, m ; \alpha, \beta)$. Since $\left(l_{n}\right)_{n \in \mathbb{N}}$ is bounded and $\overline{\mathcal{V}}_{M}^{m}$ is compact, it follows from the definition of $l_{n}$ that along an appropriate subsequence both $l_{n} \rightarrow l_{\infty} \geq t$ and $\rho_{n} \rightarrow \rho_{\infty} \in \mathcal{R}_{p, M}^{m}$ as $n \rightarrow \infty$. Hence, the proof will be complete once we show that

$$
l_{\infty} \leq \sup _{u \in \mathcal{B} \overline{\mathcal{V}}_{M}^{m}} V\left(\rho_{\infty}, u\right)
$$

because the right-hand side in (6.62) is bounded from above by $f(M, m ; \alpha, \beta)$.

Recall (4.21) and, for $\Theta \in \overline{\mathcal{V}}_{M}^{m}$ and $y \in \mathbb{R}$, define

$$
u_{\Theta}^{M, m}(y)= \begin{cases}t_{\Theta} & \text { if } \partial_{u}^{+}(u \psi(\Theta, u))\left(t_{\Theta}\right) \leq y \\ m & \text { if } \partial_{u}^{-}(u \psi(\Theta, u))(m) \geq y \\ z & \text { otherwise, with } z \text { such that } \\ & \partial_{u}^{-}(u \psi(\Theta, u))(z) \geq y \geq \partial_{u}^{+}(u \psi(\Theta, u))(z),\end{cases}
$$

where $z$ is unique by strict concavity of $u \rightarrow u \psi(\Theta, u)$ (see Lemma C.2).

Lemma 6.8. (i) For all $y \in \mathbb{R}$ and $(M, m) \in \mathrm{EIGH}, \Theta \mapsto u_{\Theta}^{M, m}(y)$ is continuous on $\left(\overline{\mathcal{V}}_{M}^{m}, d_{M}\right)$, where $d_{M}$ is defined in (C.7) in Appendix C.

(ii) For all $(M, m) \in \mathrm{EIGH}$ and $\Theta \in \overline{\mathcal{V}}_{M}^{m}, y \mapsto u_{\Theta}^{M, m}(y)$ is continuous on $\mathbb{R}$.

Proof: The proof uses the strict concavity of $u \rightarrow u \psi(\Theta, u)$ (see Lemma C.2).

(i) The proof is by contradiction. Pick $y \in \mathbb{R}$, and pick a sequence $\left(\Theta_{n}\right)_{n \in \mathbb{N}}$ in $\overline{\mathcal{V}}_{M}^{m}$ such that $\lim _{n \rightarrow \infty} \Theta_{n}=\Theta_{\infty} \in \overline{\mathcal{V}}_{M}^{m}$. Suppose that $u_{\Theta_{n}}^{M, m}(y)$ does not tend to $u_{\Theta \infty}^{M, m}(y)$ as $n \rightarrow \infty$. Then, by choosing an appropriate subsequence, we may assume 
that $\lim _{n \rightarrow \infty} u_{\Theta_{n}}^{M, m}(y)=u_{1} \in\left[t_{\Theta_{\infty}}, m\right]$ with $u_{1}<u_{\Theta_{\infty}}^{M, m}(y)$. The case $u_{1}>u_{\Theta_{\infty}}^{M, m}(y)$ can be handled similarly.

Pick $u_{2} \in\left(u_{1}, u_{\Theta_{\infty}}^{M, m}(y)\right)$. For $n$ large enough, we have $u_{\Theta_{n}}^{M, m}(y)<u_{2}<u_{\Theta_{\infty}}^{M, m}(y)$. By the definition of $u_{\Theta_{n}}^{M, m}(y)$ in (6.63) and the strict concavity of $u \mapsto u \psi\left(\Theta_{n}, u\right)$ we have, for $n$ large enough,

$$
\partial_{u}^{+}\left(u \psi\left(\Theta_{n}, u\right)\right)\left(u_{\Theta_{n}}^{M, m}(y)\right)>\frac{u_{\Theta_{\infty}}^{M, m}(y) \psi\left(\Theta_{n}, u_{\Theta_{\infty}}^{M, m}(y)\right)-u_{2} \psi\left(\Theta_{n}, u_{2}\right)}{u_{\Theta_{\infty}}^{M, m}(y)-u_{2}} .
$$

Let $n \rightarrow \infty$ in (6.64) and use the strict concavity once again, to get

$$
\liminf _{n \rightarrow \infty} \partial_{u}^{+}\left(u \psi\left(\Theta_{n}, u\right)\right)\left(u_{\Theta_{n}}^{M, m}(y)\right)>\partial_{u}^{-}\left(u \psi\left(\Theta_{\infty}, u\right)\right)\left(u_{\Theta_{\infty}}^{M, m}(y)\right)
$$

If $u_{\Theta}^{M, m}(y) \in\left(t_{\Theta_{\infty}}, m\right]$, then (6.63) implies that the right-hand side of (6.65) is not smaller than $y$. Hence (6.65) yields that $\partial_{u}^{+}\left(u \psi\left(\Theta_{n}, u\right)\right)\left(u_{\Theta_{n}}^{M, m}(y)\right)>y$ for $n$ large enough, which implies that $u_{\Theta_{n}}^{M, m}(y)=m$ by (6.63). However, the latter inequality contradicts the fact that $u_{\Theta_{n}}^{M, m}(y)<u_{2}<u_{\Theta \infty}^{M, m}(y)$ for $n$ large enough. If $u_{\Theta_{\infty}}^{M, m}(y)=t_{\Theta_{\infty}}$, then we note that $\lim _{n \rightarrow \infty} t_{\Theta_{n}}=t_{\Theta_{\infty}}$, which again contradicts that $t_{\Theta_{n}} \leq u_{\Theta_{n}}^{M, m}(y)<u_{2}<u_{\Theta_{\infty}}^{M, m}(y)$ for $n$ large enough.

(ii) The proof is again by contradiction. Pick $\Theta \in \overline{\mathcal{V}}_{M}^{m}$, and pick an infinite sequence $\left(y_{n}\right)_{n \in \mathbb{N}}$ such that $\lim _{n \rightarrow \infty} y_{n}=y_{\infty} \in \mathbb{R}$ and such that $u_{\Theta}^{M, m}\left(y_{n}\right)$ does not converge to $u_{\Theta}^{M, m}\left(y_{\infty}\right)$. Then, by choosing an appropriate subsequence, we may assume that there exists a $u_{1}<u_{\Theta}^{M, m}\left(y_{\infty}\right)$ such that $\lim _{n \rightarrow \infty} u_{\Theta}^{M, m}\left(y_{n}\right)=u_{1}$. The case $u_{1}>u_{\Theta}^{M, m}\left(y_{\infty}\right)$ can be treated similarly.

Pick $u_{2}, u_{3} \in\left(u_{1}, u_{\Theta}^{M, m}\left(y_{\infty}\right)\right)$ such that $u_{2}<u_{3}$. Then, for $n$ large enough, we have

$$
t_{\Theta} \leq u_{\Theta}^{M, m}\left(y_{n}\right)<u_{2}<u_{3}<u_{\Theta}^{M, m}\left(y_{\infty}\right) \leq m
$$

Combining (6.63) and (6.66) with the strict concavity of $u \mapsto u \psi(\Theta, u)$ we get, for $n$ large enough,

$$
y_{n}>\partial_{u}^{+}(u \psi(\Theta, u))\left(u_{2}\right)>\partial_{u}^{-}(u \psi(\Theta, u))\left(u_{3}\right)>y_{\infty},
$$

which contradicts $\lim _{n \rightarrow \infty} y_{n}=y_{\infty}$.

We resume the line of proof. Recall that $\rho_{n, 1}, n \in \mathbb{N}$, charges finitely many $\Theta \in \overline{\mathcal{V}}_{M}^{m}$. Therefore the continuity and the strict concavity of $u \mapsto u \psi(\Theta, u)$ on $\left[t_{\Theta}, m\right]$ for all $\Theta \in \overline{\mathcal{V}}_{M}^{m}$ (see Lemma C.4) imply that the supremum in (6.61) is attained at some $u_{n}^{M, m} \in \mathcal{B}_{\overline{\mathcal{V}}_{M}^{m}}$ that satisfies $u_{n}^{M, m}(\Theta)=u_{\Theta}^{M, m}\left(l_{n}\right)$ for $\Theta \in \overline{\mathcal{V}}_{M}^{m}$. Set $u_{\infty}^{M, m}(\Theta)=u_{\Theta}^{M, m}\left(l_{\infty}\right)$ for $\Theta \in \overline{\mathcal{V}}_{M}^{m}$ and note that $\left(l_{n}\right)_{n \in \mathbb{N}}$ may be assumed to be monotone, say, non-decreasing. Then the concavity of $u \mapsto u \psi(\Theta, u)$ for $\Theta \in \overline{\mathcal{V}}_{M}^{m}$ implies that $\left(u_{n}^{M, m}\right)_{n \in \mathbb{N}}$ is a non-increasing sequence of functions on $\overline{\mathcal{V}}_{M}^{m}$. Moreover, $\overline{\mathcal{V}}_{M}^{m}$ is a compact set and, by Lemma 6.8(ii), $\lim _{n \rightarrow \infty} u_{n}^{M, m}(\Theta)=u_{\infty}^{M, m}(\Theta)$ for $\Theta \in \overline{\mathcal{V}}_{M}^{m}$. Therefore Dini's theorem implies that $\lim _{n \rightarrow \infty} u_{n}^{M, m}=u_{\infty}^{M, m}$ uniformly 
on $\overline{\mathcal{V}}_{M}^{m}$. We estimate

$$
\begin{aligned}
& \left|l_{n}-\int_{\overline{\mathcal{V}}_{M}^{m}} u_{\infty}^{M, m}(\Theta) \psi\left(\Theta, u_{\infty}^{M, m}(\Theta)\right) \rho_{\infty}(d \Theta)\right| \\
& \leq \int_{\overline{\mathcal{V}}_{M}^{m}}\left|u_{n}^{M, m}(\Theta) \psi\left(\Theta, u_{n}^{M, m}(\Theta)\right)-u_{\infty}^{M, m}(\Theta) \psi\left(\Theta, u_{\infty}^{M, m}(\Theta)\right)\right| \rho_{n}(d \Theta) \\
& +\left|\int_{\overline{\mathcal{V}}_{M}^{m}} u_{\infty}^{M, m}(\Theta) \psi\left(\Theta, u_{\infty}^{M, m}(\Theta)\right) \rho_{n}(d \Theta)-\int_{\overline{\mathcal{V}}_{M}^{m}} u_{\infty}^{M, m}(\Theta) \psi\left(\Theta, u_{\infty}^{M, m}(\Theta)\right) \rho_{\infty}(d \Theta)\right| .
\end{aligned}
$$

The second term in the right-hand side of (6.68) tends to zero as $n \rightarrow \infty$ because, by Lemma 6.8(i), $\Theta \mapsto u_{\infty}^{M, m}(\Theta)$ is continuous on $\overline{\mathcal{V}}_{M}^{m}$ and because $\rho_{n}$ converges in law to $\rho_{\infty}$ as $n \rightarrow \infty$. The first term in the right-hand side of (6.68) tends to zero as well, because $(\Theta, u) \mapsto u \psi(\Theta, u)$ is uniformly continuous on $\overline{\mathcal{V}}_{M}^{*, m}$ (see Lemma C.3) and because we have proved above that $u_{n}^{M, m}$ converges to $u_{\infty}^{M, m}$ uniformly on $\overline{\mathcal{V}}_{M}^{m}$. This proves (6.62), and so Step 3 is complete.

6.2.4. Step 4. In this step we prove that

$$
\limsup _{n \rightarrow \infty} f_{3, n}^{\Omega}(M, m ; \alpha, \beta) \geq f(M, m ; \alpha, \beta) \text { for } \mathbb{P}-\text { a.e. } \Omega \text {. }
$$

Note that the proof will be complete once we show that

$$
\limsup _{n \rightarrow \infty} f_{3, n}^{\Omega}(M, m, \alpha, \beta) \geq V(\rho, u) \text { for } \rho \in \mathcal{R}_{p, M}^{m}, u \in \mathcal{B}_{\overline{\mathcal{V}}_{M}^{m}} .
$$

Pick $\Omega \in\{A, B\}^{\mathbb{N}_{0} \times \mathbb{Z}}, \rho \in \mathcal{R}_{p, M}^{\Omega, m}$ and $u \in \mathcal{B}_{\overline{\mathcal{V}}_{M}^{m}}$. By the definition of $\mathcal{R}_{p, M}^{\Omega, m}$, there exists a strictly increasing subsequence $\left(n_{k}\right)_{k \in \mathbb{N}} \in \mathbb{N}^{\mathbb{N}}$ such that, for all $k \in \mathbb{N}$, there exists an

$$
N_{k} \in\left\{\frac{n_{k}}{m L_{n_{k}}}, \ldots, \frac{n_{k}}{L_{n_{k}}}\right\}
$$

a $\Theta_{\text {traj }}^{k} \in \widetilde{\mathcal{D}}_{L_{n_{k}}, N_{k}}^{M}$ and a $x^{k} \in \mathcal{X}_{\Theta_{\text {traj }}^{k}, \Omega}^{M, m}$ such that $\rho_{k}={ }^{\text {def }} \rho_{\Theta_{\text {traj }}^{k}, x^{k}}^{\Omega}$ (see (6.52)) converges in law to $\rho$ as $k \rightarrow \infty$. Recall (6.28), and note that

$$
\Xi_{j}^{k}=\left(\Delta \Pi_{j}^{k}, b_{j}^{k}, b_{j+1}^{k}\right), \quad j=0, \ldots, N_{k}-1,
$$

with $\Delta \Pi_{j}^{k} \in\{-M, \ldots, M\}$ and $b_{j}^{k} \in(0,1] \cap \frac{\mathbb{N}}{L_{n_{k}}}$ for $j=0, \ldots, N_{k}$. For ease of notation we define

$$
\Theta_{j}^{k}=\left(\Omega\left(j, \Pi_{j}^{k}+\cdot\right), \Xi_{j}^{k}, x_{j}^{k}\right) \quad \text { with } \quad \Pi_{j}^{k}=\sum_{i=0}^{j-1} \Delta \Pi_{i}^{k}, \quad j=0, \ldots, N_{k}-1,
$$

and

$$
v_{k}=N_{k} \int_{\Theta \in \mathcal{V}_{M}^{m}} u_{\Theta} \rho_{k, 1}(d \Theta)=\sum_{j=0}^{N_{k}-1} u_{\Theta_{j}^{k}},
$$

where we recall that $u=\left(u_{\Theta}\right)_{\Theta \in \overline{\mathcal{V}}_{M}^{m}}$ was fixed at the beginning of the section.

Next, we recall that $\lim _{n \rightarrow \infty} L_{n} / n=0$ and that $L_{n}$ is non-decreasing (see (2.5)). Thus, $L_{n}$ is constant on intervals. On those intervals, $n / L_{n}$ takes constant increments. The latter implies that there exists an $\widetilde{n}_{k} \in \mathbb{N}$ satisfying

$$
0 \leq v_{k}-\frac{\widetilde{n}_{k}}{L_{\tilde{n}_{k}}} \leq \frac{1}{L_{\tilde{n}_{k}}} \quad \text { and therefore } \quad 0 \leq v_{k} L_{\widetilde{n}_{k}}-\widetilde{n}_{k} \leq 1
$$


Next, for $j=0, \ldots, N_{k}-1$ we pick $\overline{b_{j}^{k}} \in(0,1] \cap \frac{N}{L_{\tilde{n}_{k}}}$ such that $\left|\overline{b_{j}^{k}}-b_{j}^{k}\right| \leq \frac{1}{L_{\tilde{n}_{k}}}$, define

and pick

$$
\overline{\Xi_{j}^{k}}=\left(\Delta \Pi_{j}^{k}, \overline{b_{j}^{k}}, \overline{b_{j+1}^{k}}\right), \quad \overline{\Theta_{j}^{k}}=\left(\Omega\left(j, \Pi_{j}^{k}+\cdot\right), \overline{\Xi_{j}^{k}}, x_{j}^{k}\right),
$$

$$
s_{j}^{k} \in t \overline{\Theta_{j}^{k}}+\frac{2 \mathbb{N}}{L_{\widetilde{n}_{k}}} \quad \text { such that }\left|s_{j}^{k}-u_{\Theta_{j}^{k}}\right| \leq 2 / L_{\widetilde{n}_{k}} .
$$

We use (6.74) to write

$$
L_{\widetilde{n}_{k}} \sum_{j=0}^{N_{k}-1} s_{j}^{k}=L_{\widetilde{n}_{k}}\left(v_{k}+\sum_{j=0}^{N_{k}-1}\left(s_{j}^{k}-u_{\Theta_{j}^{k}}\right)\right)=L_{\widetilde{n}_{k}}(I+I I) .
$$

Next, we note that (6.75) and (6.77) imply that $\left|L_{\widetilde{n}_{k}} I-\widetilde{n}_{k}\right| \leq 1$ and $\left|L_{\widetilde{n}_{k}} I I\right| \leq 2 N_{k}$. The latter in turn implies that, by adding or subtracting at most 3 steps per column, the quantities $s_{j}^{k}$ for $j=0, \ldots, N_{k}-1$ can be chosen in such a way that $\sum_{j=0}^{N_{k}-1} s_{j}^{k}=\widetilde{n}_{k} / L_{\widetilde{n}_{k}}$.

Next, set

$$
\overline{\Theta_{\text {traj }}^{k}}=\left(\overline{\Xi_{j}^{k}}\right)_{j=0}^{N_{k}-1} \in \widetilde{\mathcal{D}}_{L_{\tilde{n}_{k}}, N_{k}}^{M}, \quad s^{k}=\left(s_{j}^{k}\right)_{j=0}^{N_{k}-1} \in \mathcal{U}_{\Theta_{\text {traj }}^{k}, x^{k}, \widetilde{n}_{k}}^{M, L_{\tilde{n}_{k}}^{k}},
$$

and recall (6.48) to get $f_{3}^{\Omega}\left(\widetilde{n}_{k}, M\right) \geq R_{k}$ with

$$
R_{k}=\frac{L_{\widetilde{n}_{k}} H^{\Omega}\left(\overline{\Theta_{\mathrm{traj}}^{k}}, x^{k}, s^{k}\right)}{\widetilde{n}_{k}}=\frac{\sum_{j=0}^{N_{k}-1} s_{j}^{k} \psi\left(\overline{\Theta_{j}^{k}}, s_{j}^{k}\right)}{\sum_{j=0}^{N_{k}-1} s_{j}^{k}}=\frac{R_{\mathrm{nu}}^{k}}{R_{\mathrm{de}}^{k}} .
$$

Further set

$$
R_{k}^{\prime}=\frac{R_{\mathrm{nu}}^{\prime k}}{R_{\mathrm{de}}^{\prime k}}=\frac{\int_{\overline{\mathcal{V}}_{M}^{m}} u_{\Theta} \psi\left(\Theta, u_{\Theta}\right) \rho_{k}(d \Theta)}{\int_{\overline{\mathcal{V}}_{M}^{m}}^{m} u_{\Theta} \rho_{k}(d \Theta)}
$$

and note that $\lim _{k \rightarrow \infty} R_{k}^{\prime}=V(\rho, u)$, since $\lim _{k \rightarrow \infty} \rho_{k}=\rho$ by assumption and $\Theta \mapsto u_{\Theta}$ is continuous on $\mathcal{V}_{M}^{m}$. We note that $R_{k}^{\prime}$ can be rewritten in the form

$$
R_{k}^{\prime}=\frac{R_{\mathrm{nu}}^{\prime k}}{R_{\mathrm{de}}^{\prime k}}=\frac{\sum_{j=0}^{N_{k}-1} u_{\Theta_{j}^{k}} \psi\left(\Theta_{j}^{k}, u_{\Theta_{j}^{k}}\right)}{\sum_{j=0}^{N_{k}-1} u_{\Theta_{j}^{k}}} .
$$

Now recall that $\lim _{k \rightarrow \infty} n_{k}=\infty$. Since $N_{k} \geq n_{k} / M L_{n_{k}}$, it follows that $\lim _{k \rightarrow \infty} N_{k}=\infty$ as well. Moreover, $N_{k} \leq \widetilde{n}_{k} / L_{\widetilde{n}_{k}}$ with $\lim _{k \rightarrow \infty} \widetilde{n}_{k}=\infty$. Therefore (6.74-6.75) allow us to conclude that $R_{\mathrm{de}}^{k}=\widetilde{n}_{k} / L_{\widetilde{n}_{k}}=R_{\mathrm{de}}^{\prime k}[1+o(1)]$.

Next, note that $\mathcal{H}_{M}$ is compact, and that $(\Theta, u) \mapsto u \psi(\Theta, u)$ is continuous on $\mathcal{H}_{M}$ and therefore is uniformly continuous. Consequently, for all $\varepsilon>0$ there exists an $\eta>0$ such that, for all $(\Theta, u),\left(\Theta^{\prime}, u^{\prime}\right) \in \mathcal{H}_{M}$ satisfying $\left|\Theta-\Theta^{\prime}\right| \leq \eta$ and $\left|u-u^{\prime}\right| \leq \eta$,

$$
\left|u \psi(\Theta, u)-u^{\prime} \psi\left(\Theta^{\prime}, u^{\prime}\right)\right| \leq \varepsilon .
$$

We recall (6.76), which implies that $d_{M}\left(\overline{\Theta_{j}^{k}}, \Theta_{j}\right) \leq 2 / L_{\widetilde{n}_{k}}$ for all $j \in\left\{0, \ldots, N_{k}-1\right\}$, we choose $k$ large enough to ensure that $2 / L_{\widetilde{n}_{k}} \leq \eta$, and we use (6.83), to obtain

$$
R_{\mathrm{nu}}^{k}=\sum_{j=0}^{N_{k}-1} s_{j}^{k} \psi\left(\overline{\Theta_{j}^{k}}, s_{j}^{k}\right)=\sum_{j=0}^{N_{k}-1} u_{\Theta_{j}^{k}} \psi\left(\Theta_{j}^{k}, u_{\Theta_{j}^{k}}\right)+T=R_{\mathrm{nu}}^{\prime k}+T
$$


with $|T| \leq \varepsilon N_{k}$. Since $\lim _{k \rightarrow \infty} R_{k}^{\prime}=V(\rho, u)$ and $\sum_{j=0}^{N_{k}-1} u_{\Theta_{j}^{k}}=v_{k} \geq \widetilde{n}_{k} / L_{\widetilde{n}_{k}}$ (see $(6.75))$, if $V(\rho, u) \neq 0$, then $\left|R_{\text {nu }}^{\prime k}\right| \geq$ Cst. $\widetilde{n}_{k} / L_{\widetilde{n}_{k}}$, whereas $|T| \leq \varepsilon N_{k} \leq \varepsilon \widetilde{n}_{k} / L_{\widetilde{n}_{k}}$ for $k$ large enough. Hence $T=o\left(R_{\mathrm{nu}}^{\prime k}\right)$ and

$$
\frac{R_{\mathrm{nu}}^{k}}{R_{\mathrm{de}}^{k}}=\frac{R_{\mathrm{nu}}^{\prime k}[1+o(1)]}{R_{\mathrm{de}}^{\prime k}[1+o(1)]} \rightarrow V(\rho, u), \quad k \rightarrow \infty .
$$

Finally, if $V(\rho, u)=0$, then $R_{\mathrm{nu}}^{\prime k}=o\left(R_{\mathrm{de}}^{\prime k}\right)$ and $T=o\left(R_{\mathrm{de}}^{\prime k}\right)$, so that $R_{k}$ tends to 0 . This completes the proof of Step 4.

6.2.5. Step 5. In this step we prove (6.24), suppressing the $(\alpha, \beta)$-dependence from the notation. For $\Omega \in\{A, B\}^{\mathbb{N}_{0} \times \mathbb{Z}^{2}}, n \in \mathbb{N}, N \in\left\{n / m L_{n}, \ldots, n / L_{n}\right\}$ and $r \in$ $\{-N M, \ldots, N M\}$, we recall $(6.28)$ and define

$$
\widetilde{\mathcal{D}}_{L, N}^{M, m, r}=\left\{\Theta_{\text {traj }} \in \widetilde{\mathcal{D}}_{L, N}^{M, m}: \Pi_{N}=r\right\},
$$

where we recall that $\Pi_{N}=\sum_{j=0}^{N-1} \Delta \Pi_{j}$. We set

$$
f_{3, n}^{\Omega}(M, m, N, r)=\frac{1}{n} \log Z_{3, n, L_{n}}^{\Omega}(N, M, m, r)
$$

with

$$
Z_{3, n, L_{n}}^{\Omega}(N, M, m, r)=\sum_{\Theta_{\text {traj }} \in \widetilde{\mathcal{D}}_{L_{n}, N}^{M, m, r}} \sum_{x \in \mathcal{X}_{\Theta_{\text {traj }}^{M, m}, \Omega}} \sum_{u \in \mathcal{U}_{\Theta_{\text {traj }}^{M, m}}^{M, L_{n}}} A_{3},
$$

where $A_{3}$ is defined in (6.49). We further set $f_{3}(\cdot)=\mathbb{E}_{\Omega}\left(f_{3}^{\Omega}(\cdot)\right)$.

6.2.6. Step 6: Concentration of measure. In the first part of this step we prove that for all $(M, m, \alpha, \beta) \in \mathrm{EIGH} \times \mathrm{CONE}$ there exist $c_{1}, c_{2}>0$ (depending on $(M, m, \alpha, \beta)$ only) such that, for all $n \in \mathbb{N}, N \in\left\{n /\left(m L_{n}\right), \ldots n / L_{n}\right\}$ and $r \in\{-N M, \ldots, N M\}$,

$$
\begin{aligned}
& \mathbb{P}_{\Omega}\left(\left|f_{3, n}^{\Omega}(M, m)-f_{3, n}(M, m)\right|>\varepsilon\right) \leq c_{1} e^{-\frac{c_{2} \varepsilon^{2} n}{L_{n}}}, \\
& \mathbb{P}_{\Omega}\left(\left|f_{3, n}^{\Omega}(M, m, N, r)-f_{3, n}(M, m, N, r)\right|>\varepsilon\right) \leq c_{1} e^{-\frac{c_{2} \varepsilon^{2} n}{L_{n}}} .
\end{aligned}
$$

We only give the proof of the first inequality. The second inequality is proved in a similar manner. The proof uses Theorem D.1. Before we start we note that, for all $n \in \mathbb{N},(M, m) \in$ EIGH and $\Omega \in\{A, B\}^{\mathbb{N}_{0} \times \mathbb{Z}}, f_{3, n}^{\Omega}(M, m)$ only depends on

$$
\mathcal{C}_{0, L_{n}}^{\Omega}, \ldots, \mathcal{C}_{n / L_{n}, L_{n}}^{\Omega} \quad \text { with } \quad \mathcal{C}_{j, L_{n}}^{\Omega}=(\Omega(j, i))_{i=-n / L_{n}}^{n / L_{n}} .
$$

We apply Theorem D.1 with $\mathcal{S}=\left\{0, \ldots, n / L_{n}\right\}$, with $X_{i}=\{A, B\}^{\left\{-\frac{n}{L_{n}}, \ldots, \frac{n}{L_{n}}\right\}}$ and with $\mu_{i}$ the uniform measure on $X_{i}$ for all $i \in \mathcal{S}$. Note that $\mid f_{3, n}^{\Omega_{1}}(M, m)-$ $f_{3, n}^{\Omega_{2}}(M, m) \mid \leq 2 C_{\mathrm{uf}}(\alpha) m \frac{L_{n}}{n}$ for all $i \in \mathcal{S}$ and all $\Omega_{1}, \Omega_{2}$ satisfying $\mathcal{C}_{j, n}^{\Omega_{1}}=\mathcal{C}_{j, n}^{\Omega_{2}}$ for all $j \neq i$. After we set $c=2 C_{\mathrm{uf}}(\alpha) m$ we can apply Theorem D.1 with $D=c^{2} L_{n} / n$ to get (6.89).

Next, we note that the first inequality in (6.89), the Borel-Cantelli lemma and the fact that $\lim _{n \rightarrow \infty} n / L_{n} \log n=\infty$ (recall (2.5)) imply that, for all $(M, m) \in \mathrm{EIGH}$,

$$
\lim _{n \rightarrow \infty}\left[f_{3, n}^{\Omega}(M, m)-f_{3, n}(M, m)\right]=0 \quad \text { for } \mathbb{P} \text { - a.e. } \Omega .
$$

Therefore (6.24) will be proved once we show that

$$
\liminf _{n \rightarrow \infty} f_{3, n}(M, m)=\limsup _{n \rightarrow \infty} f_{3, n}(M, m) .
$$


To that end, we first prove that, for all $n \in \mathbb{N}$ and all $(M, m) \in$ EIGH, there exist an $N_{n} \in\left\{n / m L_{n}, \ldots, n / L_{n}\right\}$ and an $r_{n} \in\left\{-M N_{n}, \ldots, M N_{n}\right\}$ such that

$$
\lim _{n \rightarrow \infty}\left[f_{3, n}(M, m)-f_{3, n}\left(M, m, N_{n}, r_{n}\right)\right]=0 .
$$

The proof of (6.93) is done as follows. Pick $\varepsilon>0$, and for $\Omega \in\{A, B\}^{\mathbb{N}_{0} \times \mathbb{Z}}, n \in \mathbb{N}$ and $(M, m) \in$ EIGH, denote by $N_{n}^{\Omega}$ and $r_{n}^{\Omega}$ the maximizers of $f_{3, n}^{\Omega}(M, m, N, r)$. Then

$$
f_{3, n}^{\Omega}\left(M, m, N_{n}^{\Omega}, r_{n}^{\Omega}\right) \leq f_{3, n}^{\Omega}(M, m) \leq \frac{1}{n} \log \left(\frac{n^{2}}{L_{n}^{2}}\right)+f_{3, n}^{\Omega}\left(M, m, N_{n}^{\Omega}, r_{n}^{\Omega}\right),
$$

so that, for $n$ large enough and every $\Omega$,

$$
0 \leq f_{3, n}^{\Omega}(M, m)-f_{3, n}^{\Omega}\left(M, m, N_{n}^{\Omega}, r_{n}^{\Omega}\right) \leq \varepsilon .
$$

For $n \in \mathbb{N}, N \in\left\{n / m L_{n}, \ldots, n / L_{n}\right\}$ and $r \in\{-N M, \ldots, N M\}$, we set

$$
A_{n, N, r}=\left\{\Omega:\left(N_{n}^{\Omega}, r_{n}^{\Omega}\right)=(N, r)\right\} .
$$

Next, denote by $N_{n}, r_{n}$ the maximizers of $\mathbb{P}\left(A_{n, N, r}\right)$. Note that (6.93) will be proved once we show that, for all $\varepsilon>0,\left|f_{3, n}(M, m)-f_{3, n}\left(M, m, N_{n}, r_{n}\right)\right| \leq \varepsilon$ for $n$ large enough. Further note that $\mathbb{P}\left(A_{n, N_{n}, r_{n}}\right) \geq L_{n}^{2} / n^{2}$ for all $n \in \mathbb{N}$. For every $\Omega$ we can therefore estimate

$$
\left|f_{3, n}(M, m)-f_{3, n}\left(M, m, N_{n}, r_{n}\right)\right| \leq I+I I+I I I
$$

with

$$
\begin{aligned}
& I=\left|f_{3, n}(M, m)-f_{3, n}^{\Omega}(M, m)\right| \\
& I I=\left|f_{3, n}^{\Omega}(M, m)-f_{3, n}^{\Omega}\left(M, m, N_{n}, r_{n}\right)\right|, \\
& I I I=\left|f_{3, n}^{\Omega}\left(M, m, N_{n}, r_{n}\right)-f_{3, n}\left(M, m, N_{n}, r_{n}\right)\right| .
\end{aligned}
$$

Hence, the proof of (6.93) will be complete once we show that, for $n$ large enough, there exists an $\Omega_{\varepsilon, n}$ for which $I, I I$ and $I I I$ in (6.98) are bounded from above by $\varepsilon / 3$.

To that end, note that, because of (6.89), the probabilities $\mathbb{P}(\{I>\varepsilon / 3\})$ and $\mathbb{P}(\{I I I>\varepsilon / 3\})$ are bounded from above by $c_{1} e^{-c_{2} \varepsilon^{2} n / 9 L_{n}}$, while

$$
\mathbb{P}(\{I I>\varepsilon\}) \leq \mathbb{P}\left(A_{n, N_{n}, r_{n}}^{c}\right) \leq 1-\left(L_{n}^{2} / n^{2}\right), \quad n \in \mathbb{N} .
$$

Since $\lim _{n \rightarrow \infty} n / L_{n} \log n=\infty$, we have $\mathbb{P}(\{I, I I, I I I \leq \varepsilon / 3\})>0$ for $n$ large enough. Consequently, the set $\{I, I I, I I I \leq \varepsilon / 3\}$ is non-empty and (6.93) is proven.

6.2.7. Step 7: Convergence. It remains to prove (6.92). Assume that there exist two strictly increasing subsequences $\left(n_{k}\right)_{k \in \mathbb{N}}$ and $\left(t_{k}\right)_{k \in \mathbb{N}}$ and two limits $l_{2}>l_{1}$ such that $\lim _{k \rightarrow \infty} f_{3, n_{k}}(M, m)=l_{2}$ and $\lim _{k \rightarrow \infty} f_{3, t_{k}}(M, m)=l_{1}$. By using (6.93), we have that for every $k \in \mathbb{N}$ there exist $N_{k} \in\left\{n_{k} / m L_{n_{k}}, \ldots, n_{k} / L_{n_{k}}\right\}$ and $r_{k} \in$ $\left\{-M N_{k}, \ldots, M N_{k}\right\}$ such that $\lim _{k \rightarrow \infty} f_{3, n_{k}}\left(M, m, N_{k}, r_{k}\right)=l_{2}$. Denote by

$$
\left(\Theta_{\text {traj,max }}^{k, \Omega}, x_{\text {max }}^{k, \Omega}, u_{\text {max }}^{k, \Omega}\right) \in \widetilde{\mathcal{D}}_{L_{n_{k}}, N_{k}}^{M, r_{k}} \times \mathcal{X}_{\Theta_{\text {traj,max }}^{k, \Omega}, \Omega}^{M, m} \times \mathcal{U}_{\Theta_{\text {traj,max }}^{k, \Omega,}, x_{\text {max }}^{k, m, L_{n}}, n_{k}}^{k, \Omega}
$$

the maximizer of $H^{\Omega}\left(\Theta_{\text {traj }}, x, u\right)$. We recall that $\Theta_{\text {traj }}, x$ and $u$ take their values in sets that grow subexponentially fast in $n_{k}$, and therefore

$$
\lim _{k \rightarrow \infty} \frac{L_{n_{k}}}{n_{k}} \mathbb{E}_{\Omega}\left[H^{\Omega}\left(\Theta_{\text {traj,max }}^{k, \Omega}, x_{\max }^{k, \Omega}, u_{\max }^{k, \Omega}\right)\right]=l_{2} .
$$


Since $l_{2}>l_{1}$, we can use (6.101) and the fact that $\lim _{k \rightarrow \infty} n_{k} / L_{n_{k}}=\infty$ to obtain, for $k$ large enough,

$$
\mathbb{E}_{\Omega}\left[H^{\Omega}\left(\Theta_{\text {traj,max }}^{k, \Omega}, x_{\max }^{k, \Omega}, u_{\max }^{k, \Omega}\right)\right]+(\beta-\alpha) \geq \frac{n_{k}}{L_{n_{k}}}\left(l_{1}+\frac{l_{2}-l_{1}}{2}\right) .
$$

(The term $\beta-\alpha$ in the left-hand side of (6.102) is introduced for later convenience only.) Next, pick $k_{0} \in \mathbb{N}$ satisfying (6.102), whose value will be specified later. Similarly to what we did in (6.77) and (6.78), for $\Omega \in\{A, B\}^{\mathbb{N}_{0} \times \mathbb{Z}}$ and $k \in \mathbb{N}$ we associate with

$$
\Theta_{\text {traj,max }}^{k_{0}, \Omega}=\left(\Delta \Pi_{j}^{k_{0}, \Omega}, b_{0, j}^{k_{0}, \Omega}, b_{1, j}^{k_{0}, \Omega}\right)_{j=0}^{N_{k_{0}}-1} \in \widetilde{\mathcal{D}}_{L_{n_{k_{0}}}, N_{k_{0}}}^{M, r_{k_{0}}}
$$

and

$$
x_{\max }^{k_{0}, \Omega}=\left(x_{j}^{k_{0}, \Omega}\right)_{j=0}^{N_{k_{0}}-1} \in \mathcal{X}_{\Theta_{\mathrm{traj}, \text { max }}^{k_{0}, \Omega}, \Omega}^{M, m}
$$

and

$$
u_{\max }^{k_{0}, \Omega}=\left(u_{j}^{k_{0}, \Omega}\right)_{j=0}^{N_{k_{0}}-1} \in \mathcal{U}_{\Theta_{\text {traj,max }}^{k_{0}, \Omega}, L_{\max }, n_{k_{0}}}^{M,, L_{n_{k}}}
$$

the quantities

$$
\bar{\Theta}_{\text {traj }}^{k, \Omega}=\left(\Delta \Pi_{j}^{k_{0}, \Omega}, \bar{b}_{0, j}^{k, \Omega}, \bar{b}_{1, j}^{k, \Omega}\right)_{j=0}^{N_{k_{0}}-1} \in \widetilde{\mathcal{D}}_{L_{t_{k}}, N_{k_{0}}}^{M, r_{k_{0}}}
$$

and

$$
\bar{u}^{k, \Omega}=\left(\bar{u}_{j}^{k, \Omega}\right)_{j=0}^{N_{k_{0}}-1} \in \mathcal{U}_{\bar{\Theta}_{\text {traj }}^{k, \Omega}, x_{\text {max }}^{k, \Omega}, *}^{M, m, L_{t_{k}}}
$$

(where $*$ will be specified later), so that

$\left|\bar{b}_{0, j}^{k, \Omega}-b_{0, j}^{k_{0}, \Omega}\right| \leq \frac{1}{L_{t_{k}}},\left|\bar{b}_{1, j}^{k, \Omega}-b_{1, j}^{k_{0}, \Omega}\right| \leq \frac{1}{L_{t_{k}}},\left|\bar{u}_{j}^{k, \Omega}-u_{j}^{k_{0}, \Omega}\right| \leq \frac{2}{L_{t_{k}}}, j=0, \ldots, N_{k_{0}}-1$.

Next, put $\bar{s}_{k}^{\Omega}=L_{t_{k}} \sum_{j=0}^{N_{k_{0}}-1} \bar{u}_{j}^{k, \Omega}$, which we substitute for $*$ above. The uniform continuity in Lemma C.3 allows us to claim that, for $k$ large enough and for all $\Omega$,

$$
\left|\bar{u}_{j}^{k, \Omega} \psi\left(\bar{\Theta}_{j}^{k, \Omega}, \bar{u}_{j}^{k, \Omega}\right)-u_{j}^{k_{0}, \Omega} \psi\left(\Theta_{j}^{k_{0}, \Omega}, u_{j}^{k_{0}, \Omega}\right)\right| \leq \frac{l_{2}-l_{1}}{4},
$$

where we recall that, as in (6.73), for all $j=0, \ldots, N_{k_{0}}-1$,

$$
\begin{aligned}
& \bar{\Theta}_{j}^{k, \Omega}=\left(\Omega\left(j, \Pi_{j}^{k_{0}, \Omega}+\cdot\right), \Delta \Pi_{j}^{k_{0}, \Omega}, \bar{b}_{0, j}^{k, \Omega}, \bar{b}_{1, j}^{k, \Omega}, x_{j}^{k_{0}, \Omega}\right), \\
& \Theta_{j}^{k_{0}, \Omega}=\left(\Omega\left(j, \Pi_{j}^{k_{0}, \Omega}+\cdot\right), \Delta \Pi_{j}^{k_{0}, \Omega}, b_{0, j}^{k_{0}, \Omega}, b_{1, j}^{k_{0}, \Omega}, x_{j}^{k_{0}, \Omega}\right) .
\end{aligned}
$$

Recall (6.53). An immediate consequence of (6.109) is that

$$
\left|H^{\Omega}\left(\bar{\Theta}_{\text {traj }}^{k, \Omega}, x_{\text {max }}^{k_{0}, \Omega}, \bar{u}^{k, \Omega}\right)-H^{\Omega}\left(\Theta_{\text {traj,max }}^{k_{0}, \Omega}, x_{\max }^{k_{0}, \Omega}, u_{\max }^{k_{0}, \Omega}\right)\right| \leq N_{k_{0}} \frac{l_{2}-l_{1}}{4} .
$$

Hence we can use (6.102), (6.111) and the fact that $N_{k_{0}} \leq n_{k_{0}} / L_{n_{k_{0}}}$, to conclude that, for $k$ large enough,

$$
\mathbb{E}_{\Omega}\left[H^{\Omega}\left(\bar{\Theta}_{\text {traj }}^{k, \Omega}, x_{\max }^{k_{0}, \Omega}, \bar{u}^{k, \Omega}\right)\right]+(\beta-\alpha) \geq \frac{n_{k_{0}}}{L_{n_{k_{0}}}}\left(l_{1}+\frac{l_{2}-l_{1}}{4}\right) .
$$

At this stage we add a column at the end of the group of $N_{k_{0}}$ columns in such a way that the conditions $\widehat{b}_{1, N_{k_{0}}-1}^{k, \Omega}=\widehat{b}_{0, N_{k_{0}}}^{k, \Omega}$ and $\widehat{b}_{1, N_{k_{0}}}^{k, \Omega}=1 / L_{t_{k}}$ are satisfied. We put

$$
\widehat{\Xi}_{N_{k_{0}}}^{k, \Omega}=\left(\Delta \Pi_{N_{k_{0}}}^{k_{0}, \Omega}, \widehat{b}_{0, N_{k_{0}}}^{k, \Omega}, \widehat{b}_{1, N_{k_{0}}}^{k, \Omega}\right)=\left(0, \widehat{b}_{1, N_{k_{0}}-1}^{k, \Omega}, \frac{1}{L_{t_{k}}}\right),
$$


and we let $\widehat{\Theta}_{\text {traj }}^{k, \Omega} \in \widetilde{\mathcal{D}}_{L_{t_{k}}, N_{k_{0}}+1}^{M, r_{k_{0}}}$ be the concatenation of $\bar{\Theta}_{\text {traj }}^{k, \Omega}$ (see (6.106)) and $\widehat{\Xi}_{N_{k_{0}}}^{k, \Omega}$. We let $\widehat{x}^{k_{0}, \Omega} \in \mathcal{X}_{\widehat{\Theta}_{\mathrm{traj}}^{k, \Omega}, \Omega}^{M, m}$ be the concatenation of $x_{\max }^{k_{0}, \Omega}$ and 0 . We further let

$$
\widehat{s}_{k}^{\Omega}=\bar{s}_{k}^{\Omega}+\left[1+b_{1, N_{k_{0}}-1}^{k, \Omega}-\frac{1}{L_{t_{k}}}\right] L_{t_{k}},
$$

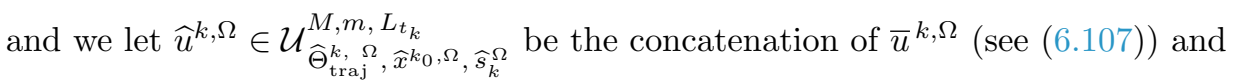

$$
\widehat{u}_{N_{k_{0}}}^{k, \Omega}=1+\left(b_{1, N_{k_{0}}-1}^{k, \Omega}-\frac{1}{L_{t_{k}}}\right) .
$$

Next, we note that the right-most inequality in (6.108), together with the fact that

$$
\sum_{j=0}^{N_{k_{0}}-1} u_{j}^{k_{0}, \Omega}=n_{k_{0}} / L_{n_{k_{0}}}
$$

allow us to asset that $\left|\bar{s}_{k}^{\Omega}-L_{t_{k}} n_{k_{0}} / L_{n_{k_{0}}}\right| \leq 2 N_{k_{0}}$. Therefore the definition of $\widehat{s}_{k}^{\Omega}$ in (6.114) implies that

$$
\widehat{s}_{k}^{\Omega}=L_{t_{k}} \frac{n_{k_{0}}}{L_{n_{k_{0}}}}+\widehat{m}_{k}^{\Omega} \quad \text { with } \quad\left|\widehat{m}_{k}^{\Omega}\right| \leq 2 N_{k_{0}}+2 L_{t_{k}} .
$$

Moreover,

$$
H^{\Omega}\left(\widehat{\Theta}_{\text {traj }}^{k, \Omega}, \widehat{x}^{k_{0}, \Omega}, \widehat{u}^{k, \Omega}\right) \geq H^{\Omega}\left(\bar{\Theta}_{\text {traj }}^{k, \Omega}, x_{\text {max }}^{k_{0}, \Omega}, \bar{u}^{k, \Omega}\right)+(\beta-\alpha),
$$

because $\widehat{u}_{N_{k_{0}}}^{k, \Omega} \leq 2$ by definition (see (6.115)) and the free energies per columns are all bounded from below by $(\beta-\alpha) / 2$. Hence, (6.112) and (6.118) give that for all $\Omega$ there exist a

$$
\widehat{\Theta}_{\text {traj }}^{k, \Omega} \in \widetilde{\mathcal{D}}_{L_{t_{k}}, N_{k_{0}}+1}^{M, r_{k_{0}}}: b_{1, N_{k_{0}}}=\frac{1}{L_{t_{k}}},
$$

an $\widehat{x}^{k_{0}, \Omega} \in \mathcal{X}_{\widehat{\Theta}_{\text {traj }}^{k, \Omega}, \Omega}^{M, m}$ and a $\widehat{u}^{k, \Omega} \in \mathcal{U}_{\widehat{\Theta}_{\text {traj }}^{k, m}, \widehat{x}^{k}, \Omega, \widehat{s}_{k}^{\Omega}}^{M, L_{t_{k}}}$ such that, for $k$ large enough,

$$
\mathbb{E}_{\Omega}\left[H\left(\widehat{\Theta}_{\text {traj }}^{k, \Omega}, \widehat{x}^{k_{0}, \Omega}, \widehat{u}^{k, \Omega}\right)\right] \geq \frac{n_{k_{0}}}{L_{n_{k_{0}}}}\left(l_{1}+\frac{l_{2}-l_{1}}{4}\right) .
$$

Next, we subdivide the disorder $\Omega$ into groups of $N_{k_{0}}+1$ consecutive columns that are successively translated by $r_{k_{0}}$ in the vertical direction, i.e., $\Omega=\left(\Omega_{1}, \Omega_{2}, \ldots\right)$ with (recall (4.13))

$$
\Omega_{j}=\left(\Omega\left(i,(j-1) r_{k_{0}}+\cdot\right)\right)_{i=(j-1)\left(N_{k_{0}}+1\right)}^{j\left(N_{k_{0}}+1\right)-1},
$$

and we let $q_{k}^{\Omega}$ be the unique integer satisfying

$$
\widehat{s}_{k}^{\Omega_{1}}+\widehat{s}_{k}^{\Omega_{2}}+\cdots+\widehat{s}_{k}^{\Omega_{q_{k}}} \leq t_{k}<\widehat{s}_{k}^{\Omega_{1}}+\cdots+\widehat{s}_{k}^{\Omega_{q_{k}+1}},
$$

where we suppress the $\Omega$-dependence of $q_{k}$. We recall that

$$
f_{3, t_{k}}^{\Omega}(M, m)=\mathbb{E}\left[\frac{1}{t_{k}} \log \sum_{N=t_{k} / m L_{t_{k}}}^{t_{k} / L_{t_{k}}} \sum_{\Theta_{\text {traj }} \in \widetilde{\mathcal{D}}_{L_{t_{k}}, N}^{M}} \sum_{x \in \mathcal{X}_{\Theta_{\text {traj }}^{M, m}}} \sum_{u \in \mathcal{U}_{\Theta_{\text {traj }}, x, t_{k}}^{M, L_{t_{k}}}} e^{L_{t_{k}}} H^{\Omega}\left(\Theta_{\text {traj }}, x, u\right)\right],
$$

set $\widetilde{t}_{k}^{\Omega}=\widehat{s}_{k}^{\Omega_{1}}+\widehat{s}_{k}^{\Omega_{2}}+\cdots+\widehat{s}_{k}^{\Omega_{q_{k}}}$, and concatenate

$$
\widehat{\Theta}_{\text {traj,tot }}^{k, \Omega}=\left(\widehat{\Theta}_{\text {traj }}^{k, \Omega_{1}}, \widehat{\Theta}_{\text {traj }}^{k, \Omega_{2}}, \ldots, \widehat{\Theta}_{\text {traj }}^{k, \Omega_{q_{k}}}\right) \in \widetilde{\mathcal{D}}_{L_{t_{k}}, q_{k}\left(N_{k_{0}}+1\right)}^{M,}
$$


and

$$
\widehat{x}_{\text {tot }}^{k, \Omega}=\left(\widehat{x}^{k_{0}, \Omega_{1}}, \widehat{x}^{k_{0}, \Omega_{2}}, \ldots, \widehat{x}^{k_{0}, \Omega_{q_{k}}}\right) \in \mathcal{X}_{\widehat{\Theta}_{\text {traj,tot }}^{k, \Omega}, \Omega}^{k, m},
$$

and

$$
\widehat{u}_{\text {tot }}^{k, \Omega}=\left(\widehat{u}^{k, \Omega_{1}}, \widehat{u}^{k, \Omega_{2}}, \ldots, \widehat{u}^{k, \Omega_{q_{k}}}\right) \in \mathcal{U}_{\widehat{\Theta}_{\text {traj,tot }}^{k, m, L_{t_{t o t}}^{k,}, \widetilde{t}_{k}^{\Omega}}}^{M,} .
$$

It still remains to complete $\widehat{\Theta}_{\text {traj,tot }}^{k, \Omega}, \widehat{x}_{\text {tot }}^{k, \Omega}$ and $\widehat{u}_{\text {tot }}^{k, \Omega}$ such that the latter becomes an element of $\mathcal{U}_{\widehat{\Theta}_{\text {traj,tot }}^{k, \Omega,}, \widehat{x}_{\text {tot }}^{k, \Omega}, t_{k}}^{M, L_{t_{k}}}$. To that end, we recall (6.122), which gives $t_{k}-\widetilde{t}_{k}^{\Omega} \leq$ $\widehat{s}_{k} \Omega_{q_{k}+1}$. Then, using (6.117), we have that there exists a $c>0$ such that

$$
t_{k}-\widetilde{t}_{k}^{\Omega} \leq c L_{t_{k}} \frac{n_{k_{0}}}{L_{n_{k_{0}}}} .
$$

Therefore we can complete $\widehat{\Theta}_{\text {traj,tot }}^{k, \Omega}, \widehat{x}_{\text {tot }}^{k, \Omega}$ and $\widehat{u}_{\text {tot }}^{k, \Omega}$ with

$$
\Theta_{\text {rest }} \in \mathcal{D}_{L_{t_{k}}, g_{k}^{\Omega}}^{M}, \quad x_{\text {rest }} \in \mathcal{X}_{\Theta_{\text {rest }}, \Omega}^{M, m}, \quad u_{\text {rest }} \in \mathcal{U}_{\Theta_{\text {rest }}, x_{\text {rest }}, t_{k}-\widetilde{t}_{k}^{\Omega}}^{M, m, L_{t_{k}}}
$$

such that, by (6.127), the number of columns $g_{k}^{\Omega}$ involved in $\Theta_{\text {rest }}$ satisfies $g_{k}^{\Omega} \leq$ $c n_{k_{0}} / L_{n_{k_{0}}}$. Henceforth $\widehat{\Theta}_{\text {traj,tot }}^{k, \Omega}, \widehat{x}_{\text {tot }}^{k, \Omega}$ and $\widehat{u}_{\text {tot }}^{k, \Omega}$ stand for the quantities defined in (6.124) and (6.126), and concatenated with $\Theta_{\text {rest }}, x_{\text {rest }}$ and $u_{\text {rest }}$ so that they become elements of

$$
\mathcal{D}_{L_{t_{k}}, q_{k}\left(N_{k_{0}}+1\right)+g_{k}^{\Omega},}^{M}, \quad \mathcal{X}_{\widehat{\Theta}_{\text {traj }, \text { tot }}^{k, m}, \Omega}^{M, m}, \quad \mathcal{U}_{\widehat{\Theta}_{\text {traj }, \text { tot }}^{k, x_{\text {tot }}, x_{k}}}^{M, m, L_{t_{k}}},
$$

respectively. By restricting the summation in (6.47) to $\widehat{\Theta}_{\text {traj,tot }}^{k, \Omega}, \widehat{x}_{\text {tot }}^{k, \Omega}$ and $\widehat{u}_{\text {tot }}^{k, \Omega}$, we get

$$
f_{3, t_{k}}(M, m) \geq \frac{L_{t_{k}}}{t_{k}} \mathbb{E}_{\Omega}\left[\sum_{j=1}^{q_{k}} H^{\Omega_{j}}\left(\widehat{\Theta}_{\text {traj }}^{k, \Omega_{j}}, \widehat{x}^{k_{0}, \Omega_{j}}, \widehat{u}^{k, \Omega_{j}}\right)+H\left(\Theta_{\text {rest }}, x_{\text {rest }}, u_{\text {rest }}\right)\right],
$$

where the term $H\left(\Theta_{\text {rest }}, x_{\text {rest }}, u_{\text {rest }}\right)$ is negligible because, by $(6.127),\left(t_{k}-\widetilde{t}_{k}^{\Omega}\right) / t_{k}$ vanishes as $k \rightarrow \infty$, while all free energies per column are bounded from below by $(\beta-\alpha) / 2$. Pick $\varepsilon>0$ and recall (6.117). Choose $k_{0}$ such that $2 L_{n_{k_{0}}} / n_{k_{0}} \leq \varepsilon / 2$ and note that, for $k$ large enough,

$$
\widehat{s}_{k}^{\Omega} \in\left[L_{t_{k}} \frac{n_{k_{0}}}{L_{n_{k_{0}}}}(1-\varepsilon), L_{t_{k}} \frac{n_{k_{0}}}{L_{n_{k_{0}}}}(1+\varepsilon)\right] .
$$

By (6.122), we therefore have

$$
q_{k} \in\left[\frac{t_{k} L_{n_{k_{0}}}}{L_{t_{k}} n_{k_{0}}} \frac{1}{1+\varepsilon}, \frac{t_{k} L_{n_{k_{0}}}}{L_{t_{k}} n_{k_{0}}} \frac{1}{1-\varepsilon}\right]=[a, b] .
$$

Recalling (6.130), we obtain

$$
\begin{aligned}
& f_{3, t_{k}}(M, m) \\
& \quad \geq \frac{L_{t_{k}}}{t_{k}} \mathbb{E}_{\Omega}\left[\sum_{j=1}^{a} H^{\Omega_{j}}\left(\widehat{\Theta}_{\text {traj }}^{k, \Omega_{j}}, \widehat{x}^{k_{0}, \Omega_{j}}, \widehat{u}^{k, \Omega_{j}}\right)-\sum_{j=a}^{b}\left|H^{\Omega_{j}}\left(\widehat{\Theta}_{\text {traj }}^{k, \Omega_{j}}, \widehat{x}^{k_{0}, \Omega_{j}}, \widehat{u}^{k, \Omega_{j}}\right)\right|\right],
\end{aligned}
$$

and, consequently,

$$
f_{3, t_{k}}(M, m) \geq \frac{L_{n_{k_{0}}}}{n_{k_{0}}(1+\varepsilon)} \mathbb{E}_{\Omega}\left[H^{\Omega}\left(\widehat{\Theta}_{\text {traj }}^{k, \Omega}, \widehat{x}^{k_{0}, \Omega}, \widehat{u}^{k, \Omega}\right)\right]-\frac{L_{t_{k}}}{t_{k}}(b-a)\left(N_{k_{0}}+1\right) m \frac{\beta-\alpha}{2},
$$


and, by (6.120),

$$
f_{3, t_{k}}(M, m) \geq \frac{l_{1}+\frac{l_{2}-l_{1}}{4}}{1+\varepsilon}-\left(\frac{1}{1-\varepsilon}-\frac{1}{1+\varepsilon}\right)(b-a) m \frac{\beta-\alpha}{2} .
$$

After taking $\varepsilon$ small enough, we may conclude that $\liminf _{k \rightarrow \infty} f_{3, t_{k}}(M, m)>l_{1}$, which completes the proof.

6.3. Proof of Proposition 6.3. Pick $(M, m) \in \mathrm{EIGH}$ and note that, for every $n \in \mathbb{N}$, the set $\mathcal{W}_{n, M}^{m}$ is contained in $\mathcal{W}_{n, M}$. Thus, by using Proposition 6.2 we obtain

$$
\begin{aligned}
\liminf _{n \rightarrow \infty} f_{1, n}^{\Omega}(M ; \alpha, \beta) & \geq \sup _{m \geq M+2} \liminf _{n \rightarrow \infty} f_{1, n}^{\Omega}(M, m ; \alpha, \beta) \\
& =\sup _{m \geq M+2} f(M, m ; \alpha, \beta) \quad \text { for } \mathbb{P}-\text { a.e. } \Omega .
\end{aligned}
$$

Therefore, the proof of Proposition 6.3 will be complete once we show that

$$
\limsup _{n \rightarrow \infty} f_{1, n}^{\Omega}(M ; \alpha, \beta) \leq \sup _{m \geq M+2} \limsup _{n \rightarrow \infty} f_{1, n}^{\Omega}(M, m ; \alpha, \beta) \quad \text { for } \mathbb{P}-\text { a.e. } \Omega .
$$

We will not prove (6.137) in full detail, but only give the main steps in the proof. The proof consists in showing that, for $m$ large enough, the pieces of the trajectory in a column that exeed $m L_{n}$ steps do not contribute substantially to the free energy.

Recall (6.27-6.32) and use (6.32) with $m=\infty$, i.e.,

$$
Z_{n, L_{n}}^{\omega, \Omega}(M)=\sum_{N=1}^{n / L_{n}} \sum_{\Theta_{\text {traj }} \in \widetilde{\mathcal{D}}_{L_{n}, N}^{M}} \sum_{x \in \mathcal{X}_{\Theta_{\text {traj }}^{M, \infty}}} \sum_{u \in \mathcal{U}_{\Theta_{\text {traj }}^{M, \infty, n}, L_{n}}} A_{1} .
$$

With each $\left(N, \Theta_{\text {traj }}, x, u\right)$ in $(6.138)$, we associate the trajectories obtained by concatenating $N$ shorter trajectories $\left(\pi_{i}\right)_{i \in\{0, \ldots, N-1\}}$ chosen in $\left(\mathcal{W}_{\Theta_{i}, u_{i}, L_{n}}\right)_{i \in\{0, \ldots, N-1\}}$, respectively. Thus, the quantity $A_{1}$ in (6.138) corresponds to the restriction of the partition function to the trajectories associated with $\left(N, \Theta_{\text {traj }}, x, u\right)$. In order to discriminate between the columns in which more than $m L_{n}$ steps are taken and those in which less are taken, we rewrite $A_{1}$ as $A_{2} \widetilde{A}_{2}$ with

$$
A_{2}=\prod_{i \in V_{u, m}} Z_{L_{n}}^{\omega_{I_{i}}}\left(\Theta_{i}, u_{i}\right), \quad \widetilde{A}_{2}=\prod_{i \in \widetilde{V}_{u, m}} Z_{L_{n}}^{\omega_{I_{i}}}\left(\Theta_{i}, u_{i}\right),
$$

with $\widetilde{u}_{i}=\sum_{k=0}^{i-1} u_{k}, \Theta_{i}=\left(\Omega\left(i, \Pi_{i}+\cdot\right), \Xi_{i}, x_{i}\right)$ and $I_{i}=\left\{\widetilde{u}_{i} L_{n}, \ldots, \widetilde{u}_{i+1} L_{n}-1\right\}$ for $i \in\{0, \ldots, N-1\}$, with $\omega_{I}=\left(\omega_{i}\right)_{i \in I}$ for $I \subset \mathbb{N}$, where $\{0, \ldots, N-1\}$ is partitioned into

$$
\widetilde{V}_{u, m} \cup V_{u, m} \text { with } \quad \widetilde{V}_{u, m}=\left\{i \in\{0, \ldots, N-1\}: u_{i}>m\right\} .
$$

For all $\left(N, \Theta_{\text {traj }}, x, u\right)$, we rewrite $\widetilde{V}_{u, m}$ in the form of an increasing sequence $\left\{i_{1}, \ldots, i_{\widetilde{k}}\right\}$ and we drop the $(u, m)$-dependence of $\widetilde{k}$ for simplicity. We also set $\widetilde{u}=u_{i_{1}}+\cdots+u_{i_{\tilde{k}}}$, which is the total number of steps taken by a trajectory associated with $\left(N, \Theta_{\text {traj }}, x, u\right)$ in those columns where more than $m L_{n}$ steps are taken. Finally, for $s \in\{1, \ldots, \widetilde{k}\}$ we partition $I_{i_{s}}$ into

$$
\begin{aligned}
J_{i_{s}} \cup \widetilde{J}_{i_{s}} \text { with } \quad J_{i_{s}} & =\left\{\widetilde{u}_{i_{s}} L_{n}, \ldots,\left(\widetilde{u}_{i_{s}}+M+2\right) L_{n}\right\}, \\
\widetilde{J}_{i_{s}} & =\left\{\left(\widetilde{u}_{i_{s}}+M+2\right) L_{n}+1, \ldots, \widetilde{u}_{i_{s}+1} L_{n}-1\right\},
\end{aligned}
$$


and we partition $\{1, \ldots, n\}$ into

$$
J \cup \widetilde{J} \text { with } \quad \widetilde{J}=\cup_{s=1}^{\widetilde{k}} \widetilde{J}_{i_{s}}, \quad J=\{1, \ldots, n\} \backslash \widetilde{J},
$$

so that $\widetilde{J}$ contains the label of the steps constituting the pieces of trajectory exeeding $(M+2) L_{n}$ steps in those columns where more than $m L_{n}$ steps are taken.

6.3.1. Step 1. In this step we replace the pieces of trajectories in the columns indexed in $\widetilde{V}_{u, m}$ by shorter trajectories of length $(M+2) L_{n}$. To that aim, for every $\left(N, \Theta_{\text {traj }}, x, u\right)$ we set

$$
\widehat{A}_{2}=\prod_{i \in \widetilde{V}_{u, m}} Z_{L_{n}}^{\omega_{J_{i}}}\left(\Theta_{i}^{\prime}, M+2\right)
$$

with $\Theta_{i}^{\prime}=\left(\Omega\left(i, \Pi_{i}+\cdot\right), \Xi_{i}, 1\right)$. We will show that for all $\varepsilon>0$ and for $m$ large enough, the event

$$
B_{n}=\left\{\omega: \widetilde{A}_{2} \leq \widehat{A}_{2} e^{3 \varepsilon n} \text { for all }\left(N, \Theta_{\text {traj }}, x, u\right)\right\}
$$

satisfies $\mathbb{P}_{\omega}\left(B_{n}\right) \rightarrow 1$ as $n \rightarrow \infty$.

Pick, for each $s \in\{1, \ldots, \widetilde{k}\}$, a trajectory $\pi_{s}$ in the set $\mathcal{W}_{\Theta_{i_{s}}, u_{i_{s}}, L_{n}}$. By concatenating them we obtain a trajectory in $\mathcal{W}_{\widetilde{u} L_{n}}$ satisfying $\pi_{\widetilde{u} L_{n}, 1}=\widetilde{k} L_{n}$. Thus, the total entropy carried by those pieces of trajectories crossing the columns indexed in $\left\{i_{1}, \ldots, i_{\widetilde{k}}\right\}$ is bounded above by

$$
\prod_{s=1}^{\widetilde{k}}\left|\mathcal{W}_{\Theta_{i_{s}}, u_{i_{s}}, L_{n}}\right| \leq\left|\left\{\pi \in \mathcal{W}_{\widetilde{u} L_{n}}: \pi_{\widetilde{u} L_{n}, 1}=\widetilde{k} L_{n}\right\}\right| .
$$

Since $\widetilde{u} / \widetilde{k} \geq m$, we can use Lemma A.2 in Appendix A to assert that, for $m$ large enough, the right-hand side of (6.145) is bounded above by $e^{\varepsilon n}$.

Moreover, we note that an $\widetilde{u} L_{n}$-step trajectory satisfying $\pi_{\widetilde{u} L_{n}, 1}=\widetilde{k} L_{n}$ makes at most $\widetilde{k} L_{n}+\widetilde{u}$ excursions in the $B$ solvent because such an excursion requires at least one horizontal step or at least $L_{n}$ vertical steps. Therefore, by using the inequalities $\widetilde{k} L_{n} \leq n / m$ and $\widetilde{u} \leq n / L_{n}$ we obtain that, for $n$ large enough, the sum of the Hamiltonians associated with $\left(\pi_{1}, \ldots, \pi_{\widetilde{k}}\right)$ is bounded from above, uniformly in $\left(N, \Theta_{\text {traj }}, x, u\right)$ and $\left(\pi_{1}, \ldots, \pi_{\widetilde{k}}\right)$, by

$$
\sum_{s=1}^{\widetilde{k}} H_{u_{i_{s}} L_{n}, L_{n}}^{\omega_{I_{i}}, \Omega\left(i_{s}, \Pi_{i_{s}}+\cdot\right)}\left(\pi_{s}\right) \leq \max \left\{\sum_{i \in I} \xi_{i}: I \in \cup_{r=1}^{2 n / m} \mathcal{E}_{n, r}\right\},
$$

with $\mathcal{E}_{n, r}$ defined in (E.1) in Appendix E and $\xi_{i}=\beta 1_{\left\{\omega_{i}=A\right\}}-\alpha 1_{\left\{\omega_{i}=B\right\}}$ for $i \in \mathbb{N}$. At this stage we use the definition in (E.3) and note that, for all $\omega \in \mathcal{Q}_{n, m}^{\varepsilon / \beta,(\alpha-\beta) / 2+\varepsilon}$, the right-hand side in (6.146) is smaller than $\varepsilon n$. Consequently, for $m$ and $n$ large enough we have that, for all $\omega \in \mathcal{Q}_{n, m}^{\varepsilon / \beta,(\alpha-\beta) / 2+\varepsilon}$,

$$
\widetilde{A}_{2} \leq e^{2 \varepsilon n} \text { for all }\left(N, \Theta_{\text {traj }}, x, u\right) .
$$

Recalling (4.37) and noting that $\tilde{k} L_{n} \leq n / m$, we can write

$$
\widehat{A}_{2} \geq e^{-\widetilde{k}(M+2) L_{n} C_{\mathrm{uf}}(\alpha)} \geq e^{-n \frac{M+2}{m} C_{\mathrm{uf}}(\alpha)},
$$

and therefore, for $m$ large enough, for all $n$ and all $\left(N, \Theta_{\text {traj }}, x, u\right)$ we have $\widehat{A}_{2} \geq$ $e^{-\varepsilon n}$. 
Finally, use (6.147) and (6.148) to conclude that, for $m$ and $n$ large enough, $\mathcal{Q}_{n, m}^{\varepsilon / \beta,(\alpha-\beta) / 2+\varepsilon}$ is a subset of $B_{n}$. Thus, Lemma E.1 ensures that, for $m$ large enough, $\lim _{n \rightarrow \infty} P_{\omega}\left(B_{n}\right)=1$.

6.3.2. Step 2. Let $\left(\widetilde{w}_{i}\right)_{i \in \mathbb{N}}$ be an i.i.d. sequence of Bernouilli trials, independent of $\omega, \Omega$. For $\left(N, \Theta_{\text {traj }}, x, u\right)$ we set $\widehat{u}=\widetilde{u}-\widetilde{k}(M+2)$. In Step 1 we have removed $\widehat{u} L_{n}$ steps from the trajectories associated with $\left(N, \Theta_{\text {traj }}, x, u\right)$ so that they have become trajectories associated with $\left(N, \Theta_{\text {traj }}, x^{\prime}, u\right)$. In this step, we will concatenate the trajectories associated with $\left(N, \Theta_{\text {traj }}, x^{\prime}, u\right)$ with an $\widehat{u} L_{n}$-step trajectory to recover a trajectory that belongs to $\mathcal{W}_{n, M}^{m}$.

For $\Omega \in\{A, B\}^{\mathbb{N}_{0} \times \mathbb{Z}}, t, N \in \mathbb{N}$ and $k \in \mathbb{Z}$, let

$$
P_{A}^{\Omega}(N, k)(t)=\frac{1}{t} \sum_{j=0}^{t-1} 1_{\{\Omega(N+j, k)=A\}}
$$

be the proportion of $A$-blocks on the $k^{\text {th }}$ line and between the $N^{\text {th }}$ and the $(N+$ $t-1)^{\text {th }}$ column of $\Omega$. Pick $\eta>0$ and $j \in \mathbb{N}$, and set

$$
S_{\eta, j}=\bigcup_{N=0}^{j} \bigcup_{k=-j}^{j} \bigcup_{t \geq \eta j}\left\{P_{A}^{\Omega}(N, k)(t) \leq \frac{p}{2}\right\} .
$$

By a straightforward application of Cramer's Theorem for i.i.d. random variables, we have that $\sum_{j \in \mathbb{N}} P_{\Omega}\left(S_{\eta, j}\right)<\infty$. Therefore, using the Borel-Cantelli Lemma, it follows that for $\mathbb{P}_{\Omega}$-a.e. $\Omega$, there exists a $j_{\eta}(\Omega) \in \mathbb{N}$ such that $\Omega \notin S_{\eta, j}$ as soon as $j \geq j_{\eta}(\Omega)$. In what follows, we consider $\eta=\varepsilon / \alpha m$ and we take $n$ large enough so that $n / L_{n} \geq j_{\varepsilon / \alpha m}(\Omega)$, and therefore $\Omega \notin S_{\frac{n}{L_{n}}, \frac{\varepsilon}{\alpha m}}$.

Pick $(N, \Theta, x, u)$ and consider one trajectory $\widehat{\pi}$, of length $\widehat{u} L_{n}$, starting from $\left(N, \Pi_{N}+b_{N}\right) L_{n}$, staying in the coarsed-grained line at height $\Pi_{N}$, crossing the $B$-blocks in a straight line and the $A$-blocks in $m L_{n}$ steps. The number of columns crossed by $\widehat{\pi}$ is denoted by $\widehat{N}$ and satisfies $\widehat{N} \geq \widehat{u} / m$. If $\widehat{u} L_{n} \leq \varepsilon n / \alpha$, then the Hamiltonian associated with $\widehat{\pi}$ is clearly larger than $-\varepsilon n$. If $\widehat{u} L_{n} \geq \varepsilon n / \alpha$ in turn, then

$$
H_{\widehat{u} L_{n}, L_{n}}^{\widetilde{w}, \Omega\left(\Pi_{N}\right)}(\widehat{\pi}) \geq-\alpha L_{n} \widehat{N}\left[1-P_{A}^{\Omega}\left(N, \Pi_{N}\right)(\widehat{N})\right]
$$

Since $N \leq n / L_{n},\left|\Pi_{N}\right| \leq n / L_{n}$ and $\widehat{N} \geq \varepsilon n /\left(\alpha m L_{n}\right)$, we can use the fact that $\Omega \notin S_{\frac{\varepsilon}{\alpha m}, \frac{n}{L_{n}}}$ to obtain

$$
P_{A}^{\Omega}\left(N, \Pi_{N}\right)(\widehat{N}) \geq \frac{p}{2}
$$

At this point it remains to bound $\widehat{N}$ from above, which is done by noting that

$$
\widehat{N}\left[m P_{A}^{\Omega}\left(N, \Pi_{N}\right)(\widehat{N})+1-P_{A}^{\Omega}\left(N, \Pi_{N}\right)(\widehat{N})\right]=\widehat{u} \leq \frac{n}{L_{n}} .
$$

Hence, using (6.152) and (6.153), we obtain $\widehat{N} \leq 2 n / p m L_{n}$ and therefore the righthand side of (6.151) is bounded from below by $-\alpha(2-p) n / p m$, which for $m$ large enough is larger than $-\varepsilon n$.

Thus, for $n$ and $m$ large enough and for all $(N, \Theta, x, u)$, we have a trajectory $\widehat{\pi}$ at which the Hamiltonian is bounded from below by $-\varepsilon n$ that can be concatenated with all trajectories associated with $\left(N, \Theta, x^{\prime}, u\right)$ to obtain a trajectory in $\mathcal{W}_{n, M}^{m}$. Consequently, recalling (6.141), for $n$ and $m$ large enough we have

$$
A_{2} \widehat{A_{2}} \leq e^{\varepsilon n} Z_{n, L_{n}}^{\left(\omega_{J}, \widetilde{\omega}\right), \Omega}(M, m) \quad \forall(N, \Theta, x, u) .
$$


6.3.3. Step 3. In this step, we average over the microscopic disorders $\omega, \widetilde{\omega}$. Use (6.154) to note that, for $n$ and $m$ large enough and all $\omega \in B_{n}$, we have

$$
Z_{n, L_{n}}^{\omega, \Omega}(M) \leq e^{4 \varepsilon n} \sum_{N=1}^{n / L_{n}} \sum_{\Theta_{\text {traj }} \in \widetilde{\mathcal{D}}_{L_{n}, N}^{M}} \sum_{x \in \mathcal{X}_{\Theta_{\text {traj }}^{M, \Omega}}^{M, \infty}} \sum_{u \in \mathcal{U}_{\Theta_{\text {traj }}^{M, \infty, n}}^{M, L_{n}}} Z_{n, L_{n}}^{\left(\omega_{J}, \widetilde{\omega}\right), \Omega}(M, m) .
$$

We use (D.3) to claim that there exists $C_{1}, C_{2}>0$ so that for all $n \in \mathbb{N}$, all $m \in \mathbb{N}$ and all $J$,

$$
\mathbb{P}_{\omega, \widetilde{\omega}}\left(\left|\frac{1}{n} \log Z_{n, L_{n}}^{\left(\omega_{J}, \widetilde{\omega}\right), \Omega}(M, m)-f_{1, n}^{\Omega}(M, m)\right| \geq \varepsilon\right) \leq C_{1} e^{-C_{2} \varepsilon^{2} n} .
$$

We set also

$$
D_{n}=\bigcap_{\left(N, \Theta_{\text {traj }}, x, u\right)}\left\{\left|\frac{1}{n} \log Z_{n, L_{n}}^{\left(\omega_{J}, \widetilde{\omega}\right), \Omega}(M, m)-f_{1, n}^{\Omega}(M, m)\right| \leq \varepsilon\right\},
$$

recall the definition of $c_{n}$ in (6.57) (used with $(M, \infty)$ ), and use (6.156) and the fact that $c_{n}$ grows subexponentially, to obtain $\lim _{n \rightarrow \infty} \mathbb{P}_{\omega, \widetilde{\omega}}\left(D_{n}^{c}\right)=0$. For all $(\omega, \widetilde{\omega})$ satisfying $\omega \in B_{n}$ and $(\omega, \widetilde{\omega}) \in D_{n}$, we can rewrite (6.155) as

$$
Z_{n, L_{n}}^{\omega, \Omega}(M) \leq c_{n} e^{n f_{1, n}^{\Omega}(M, m)+5 \varepsilon n} .
$$

As a consequence, recalling (4.37), for $m$ large enough we have

$f_{n}^{\Omega}(M ; \alpha, \beta) \leq \mathbb{P}\left(B_{n}^{c} \cup D_{n}^{c}\right) C_{\mathrm{uf}}(\alpha)+\frac{\log c_{n}}{n}+\frac{1}{n} \mathbb{E}\left(1_{\left\{B_{n} \cup D_{n}\right\}}\left(n f_{1, n}^{\Omega}(M, m)+5 \varepsilon n\right)\right)$.

Since $\mathbb{P}\left(B_{n}^{c} \cup D_{n}^{c}\right)$ and $\left(\log c_{n}\right) / n$ vanish when $n \rightarrow \infty$, it suffices to apply Proposition 6.2 and to let $\varepsilon \rightarrow 0$ to obtain (6.137). This completes the proof of Proposition 6.3.

6.4. Proof of Proposition 6.4. Note that, for all $m \geq M+2$, we have $\mathcal{R}_{p, M}^{m} \subset \mathcal{R}_{p, M}$. Moreover, any $\left(u_{\Theta}\right)_{\Theta \in \overline{\mathcal{V}}_{M}^{m}} \in \mathcal{B}_{\overline{\mathcal{V}}_{M}^{m}}$ can be extended to $\overline{\mathcal{V}}_{M}$ so that it belongs to $\mathcal{B}_{\overline{\mathcal{V}}_{M}}$. Thus,

$$
\sup _{m \geq M+2} f(M, m ; \alpha, \beta) \leq \sup _{\rho \in \mathcal{R}_{p, M}} \sup _{(u) \in \mathcal{B}_{\overline{\mathcal{V}}_{M}}} V(\rho, u) .
$$

As a consequence, it suffices to show that for all $\rho \in \mathcal{R}_{p, M}$ and $\left(u_{\Theta}\right)_{\Theta \in \overline{\mathcal{V}}_{M}} \in \mathcal{B}_{\overline{\mathcal{V}}_{M}}$,

$$
V(\rho, u) \leq \sup _{m \geq M+2} \sup _{\rho \in \mathcal{R}_{p, M}^{m}} \sup _{(u) \in \mathcal{B}_{\overline{\mathcal{V}}_{M}^{m}}^{m}} V(\rho, u)
$$

If $\int_{\overline{\mathcal{V}}_{M}} u_{\Theta} \rho(d \Theta)=\infty$, then (6.161) is trivially satisfied since $V(\rho, u)=-\infty$. Thus, we can assume that $\rho\left(\overline{\mathcal{V}}_{M} \backslash D_{M}\right)=1$, where $D_{M}=\left\{\Theta \in \overline{\mathcal{V}}_{M}: \chi_{\Theta} \in\right.$ $\left.\left\{A^{\mathbb{Z}}, B^{\mathbb{Z}}\right\}, x_{\Theta}=2\right\}$. Since $\int_{\overline{\mathcal{V}}_{M}} u_{\Theta} \rho(d \Theta)<\infty$ and since (recall $\left.(4.37)\right) \psi(\Theta, u)$ is uniformly bounded by $C_{\mathrm{uf}}(\alpha)$ on $(\Theta, u) \in \overline{\mathcal{V}}_{M}^{*}$, we have by dominated convergence that for all $\varepsilon>0$ there exists an $m_{0} \geq M+2$ such that, for all $m \geq m_{0}$,

$$
V(\rho, u) \leq \frac{\int_{\overline{\mathcal{V}}_{M}^{m}}^{m} u_{\Theta} \psi\left(\Theta, u_{\Theta}\right) \rho(d \Theta)}{\int_{\overline{\mathcal{V}}_{M}^{m}}^{m} u_{\Theta} \rho(d \Theta)}+\frac{\varepsilon}{2} .
$$

Since $\rho\left(\overline{\mathcal{V}}_{M} \backslash D_{M}\right)=1$ and since $\cup_{m \geq M+2} \overline{\mathcal{V}}_{M}^{m}=\overline{\mathcal{V}}_{M} \backslash D_{M}$, we have $\lim _{m \rightarrow \infty} \rho\left(\overline{\mathcal{V}}_{M}^{m}\right)=1$. Moreover, for all $m \geq m_{0}$ there exists a $\widehat{\rho}_{m} \in \mathcal{R}_{p, M}^{m}$ such that $\widehat{\rho}_{m}=\rho_{m}+\bar{\rho}_{m}$, with $\rho_{m}$ the restriction of $\rho$ to $\overline{\mathcal{V}}_{M}^{m}$ and $\bar{\rho}_{m}$ charging only those 
$\Theta$ satisfying $x_{\Theta}=1$. Since all $\Theta \in \overline{\mathcal{V}}_{M}$ with $x_{\Theta}=1$ also belong to $\overline{\mathcal{V}}_{M}^{M+2}$, we can state that $\bar{\rho}_{m}$ only charges $\overline{\mathcal{V}}_{M}^{M+2}$. Therefore

$$
V\left(\widehat{\rho}_{m}, u\right)=\frac{\int_{\overline{\mathcal{V}}_{M}^{m}} u_{\Theta} \psi\left(\Theta, u_{\Theta}\right) \rho(d \Theta)+\int_{\overline{\mathcal{V}}_{M}^{M+2}} u_{\Theta} \psi\left(\Theta, u_{\Theta}\right) \bar{\rho}_{m}(d \Theta)}{\int_{\overline{\mathcal{V}}_{M}^{m}} u_{\Theta} \rho(d \Theta)+\int_{\overline{\mathcal{V}}_{M}^{M+2}} u_{\Theta} \bar{\rho}_{m}(d \Theta)} .
$$

Since $\Theta \mapsto u_{\Theta}$ is continuous on $\overline{\mathcal{V}}_{M}$, there exists an $R>0$ such that $u_{\Theta} \leq R$ for all $\Theta \in \overline{\mathcal{V}}_{M}^{M+2}$. Therefore we can use (6.162) and (6.163) to obtain, for $m \geq m_{0}$,

$V\left(\widehat{\rho}_{m}, u\right) \geq\left(V(\rho, u)-\frac{\varepsilon}{2}\right) \frac{\int_{\overline{\mathcal{V}}_{M}^{m}} u_{\Theta} \rho(d \Theta)}{\int_{\overline{\mathcal{V}}_{M}^{m}} u_{\Theta} \rho(d \Theta)+\int_{\overline{\mathcal{V}}_{M}^{M+2}} u_{\Theta} \bar{\rho}_{m}(d \Theta)}-R C_{\mathrm{uf}}(\alpha)\left(1-\rho\left(\overline{\mathcal{V}}_{M}^{m}\right)\right)$.

The fact that $\bar{\rho}_{m}\left(\overline{\mathcal{V}}_{M}^{M+2}\right)=\rho\left(\overline{\mathcal{V}}_{M} \backslash \overline{\mathcal{V}}_{M}^{m}\right)$ for all $m \geq m_{0}$ implies that $\lim _{m \rightarrow \infty} \bar{\rho}_{m}\left(\mathcal{V}_{M}^{M+2}\right)=0$. Consequently, the right-hand side in (6.164) tends to $V(\rho, u)-\varepsilon / 2$ as $m \rightarrow \infty$. Thus, there exists a $m_{1} \geq m_{0}$ such that $V\left(\widehat{\rho}_{m_{1}}, u\right) \geq$ $V(\rho, u)-\varepsilon$. Finally, we note that there exists a $m_{2} \geq m_{1}+1$ such that $u_{\Theta} \leq m_{2}$ for all $\Theta \in \overline{\mathcal{V}}_{M}^{m_{1}}$, which allows us to extend $\left(u_{\Theta}\right)_{\Theta \in \overline{\mathcal{V}}_{M}^{m_{1}}}$ to $\overline{\mathcal{V}}_{M}^{m_{2}}$ such that $\left(u_{\Theta}\right)_{\Theta \in \overline{\mathcal{V}}_{M}^{m_{2}} \in}$ $\mathcal{B}_{\overline{\mathcal{V}}_{M}^{m_{2}}}$. It suffices to note that $\widehat{\rho}_{m_{1}} \in \mathcal{R}_{p, M}^{m_{1}} \subset \mathcal{R}_{p, M}^{m_{2}}$ to conclude that

$$
V(\rho, u) \leq f\left(M, m_{2} ; \alpha, \beta\right)+\varepsilon
$$

6.5. Proof of Proposition 6.5. It remains to remove the $M$-truncation from the variational formula in Proposition 6.4. To that aim it suffices to show that

$$
\limsup _{n \rightarrow \infty} f_{n}^{\Omega}(\alpha, \beta) \leq \sup _{M \geq 1} \limsup _{n \rightarrow \infty} f_{n}^{\Omega}(M ; \alpha, \beta) \quad \text { for } \mathbb{P}-\text { a.e. } \Omega .
$$

The proof of (6.166) is similar to that of (6.137) in Section 6.3. In the latter, the pieces of path inside the columns where too many steps $\left(\geq m L_{n}\right)$ were taken were replaced by a shorter path. However, the mesoscopic strategy of displacement was not changed. This is a major difference with the proof of Proposition 6.5 below, since we need to compare the contribution to the partition function of groups of trajectories that do not follow the same mesoscopic strategy of displacement.

For $\pi \in \mathcal{W}_{n}$, we recall that $N_{\pi}$ is the number of columns crossed by $\pi$ after $n$ steps. We recall (6.138-6.142) and use the same notations with $M=\infty$ to rewrite the full partition function as

$$
Z_{n, L_{n}}^{\omega, \Omega}=\sum_{N=1}^{n / L_{n}} \sum_{\Theta_{\text {traj }} \in \widetilde{\mathcal{D}}_{L_{n}, N}^{\infty}} \sum_{x \in \mathcal{X}_{\Theta_{\text {traj }}^{\infty}, \infty}} \sum_{u \in \mathcal{U}_{\Theta_{\text {traj }}^{\infty, \infty, L}, n}^{\infty, L_{n}}} A_{1} .
$$

We pick $N \in\left\{1, \ldots, n / L_{n}\right\}$, and with each $\Theta_{\text {traj }} \in \widetilde{\mathcal{D}}_{L_{n}, N}^{\infty}$ and $x \in \mathcal{X}_{\Theta_{\text {traj }}, \Omega}^{\infty, \infty}$ we associate an auxiliary mesocopic strategy denoted by $\widetilde{\Theta}_{\text {traj }} \in \widetilde{\mathcal{D}}_{L_{n}, N}^{M}$ and $\widetilde{x} \in$ $\mathcal{X}_{\widetilde{\Theta}_{\text {traj }}, \Omega}^{M, \infty}$ that is built as follows. Let $i_{1}$ be the index of the first column in which the mesoscopic displacement of $\Theta_{\text {traj }}$ is strictly larger than $M$, i.e., $\left(\left|\Delta \Pi_{i_{1}}\right|>M\right)$. Until $i_{1}$, both strategies $\left(\Theta_{\text {traj }}, x\right)$ and $\left(\widetilde{\Theta}_{\text {traj }}, \widetilde{x}\right)$ are equal, i.e.,

$$
\widetilde{\Theta}_{i}=\left(\Omega\left(i, \widetilde{\Pi}_{i}+\cdot\right), \widetilde{\Xi}_{i}, \widetilde{x}_{i}\right)=\left(\Omega\left(i, \Pi_{i}+\cdot\right), \Xi_{i}, x_{i}\right)=\Theta_{i} \quad \text { for } i \leq i_{1}-1 .
$$

The mesoscopic displacement $\Delta \Pi_{i_{1}}$ of $\Theta_{\text {traj }}$ is large and $\widetilde{\Theta}_{\text {traj }}$ starts making mesoscopic steps of size $M$ to catch up with $\Theta_{\text {traj }}$ as soon as possible. This takes $r_{1} \in \mathbb{N}$ 
columns indexed in $\left\{i_{1}, \ldots, i_{1}+r_{1}-1\right\}$ for which $\left|\Delta \widetilde{\Pi}_{i}\right|=M, \widetilde{x}_{i}=1$, except for the very last column $\left(i=i_{1}+r_{1}-1\right)$, which is used to end the catch up between $\left(\widetilde{\Theta}_{\text {traj }}, \widetilde{x}\right)$ and $\left(\Theta_{\text {traj }}, x\right)$. We note that there may be other columns among $\left\{i_{1}, \ldots, i_{1}+r_{1}-1\right\}$ in which the mesoscopic displacement of $\Theta_{\text {traj }}$ is $>M$.

After $\left(\widetilde{\Theta}_{\text {traj }}, \widetilde{x}\right)$ catches up with $\left(\Theta_{\text {traj }}, x\right)$, it remains equal to $\left(\Theta_{\text {traj }}, x\right)$ until a new column appears (indexed by $i_{2} \geq i_{1}+r_{1}$ ) with a large mesoscopic displacement, i.e., $\left|\Delta \Pi_{i_{2}}\right|>M$. Thus, $\widetilde{\Theta}_{i}=\Theta_{i}$ for $i \in\left\{i_{1}+r_{1}, \ldots i_{2}-1\right\}$, and so on. The resulting $\widetilde{\Theta}_{\text {traj }}$ and $\widetilde{x}$ belong to $\widetilde{\mathcal{D}}_{L_{n}, N}^{M}$ and $\mathcal{X}_{\widetilde{\Theta}_{\text {traj }}^{M}, \Omega}^{M, \infty}$, respectively, and $\Theta_{i}=\widetilde{\Theta}_{i}$, except on $k$ groups of consecutive columns denoted by $\left\{i_{1}, \ldots, i_{1}+r_{1}-1\right\}, \ldots,\left\{i_{k}, \ldots, i_{k}+\right.$ $\left.r_{k}-1\right\}$ and referred to as the catch-up columns in what follows. For simplicity, the dependence in $\Theta_{\text {traj }}$ of $k, i_{1}, r_{1}, \ldots, i_{k}, r_{k}$ is omitted.

We can give a crude upper bound on the number of columns on which $\widetilde{\Theta}_{\text {traj }}$ differs from $\widetilde{\Theta}_{\text {traj }}$. The sum of the absolute values of the large mesoscopic jumps (i.e., $\sum_{i=1}^{N}\left|\Delta \Pi_{i}\right| 1\left\{\left|\Delta \Pi_{i}\right|>M\right\}$ ) performed by $\Theta_{\text {traj }}$ indeed cannot exceed $n / L_{n}$. Moreover, the number of columns in which the mesoscopic displacement is larger than $M / 2$ is bounded above by $2 n / M L_{n}$, and in each catch-up column where the mesoscopic displacement of $\Theta_{\text {traj }}$ is smaller than $M / 2, \widetilde{\Theta}_{\text {traj }}$ scores at least $M / 2$ blocks in its race against $\Theta_{\text {traj }}$. Therefore, the number of catch-up columns $r_{1}+\cdots+r_{k}$ is bounded above by $4 n / M L_{n}$.

In order to discriminate between the catch-up columns and the columns on which $\Theta_{\text {traj }}$ and $\widetilde{\Theta}_{\text {traj }}$ are equal, we keep the notations of (6.140-6.142) and we rewrite $A_{1}$ as $A_{2} \widetilde{A}_{2}$ with

$$
A_{2}=\prod_{i \in V_{\Pi, M}} Z_{L_{n}}^{\omega_{I_{i}}}\left(\Theta_{i}, u_{i}\right), \quad \widetilde{A}_{2}=\prod_{i \in \widetilde{V}_{\Pi, M}} Z_{L_{n}}^{\omega_{I_{i}}}\left(\Theta_{i}, u_{i}\right),
$$

where $\{0, \ldots, N-1\}$ is partitioned into $\widetilde{V}_{\Pi, M} \cup V_{\Pi, M}$ and $\widetilde{V}_{\Pi, M}=\cup_{s=1}^{k}\left\{i_{s}, \ldots, i_{s}+\right.$ $\left.r_{s}-1\right\}$ gathers the indices of the $k$ groups of catch-up columns.

We also set $\bar{u}_{s}=u_{i_{s}}+\cdots+u_{i_{s}+r_{s}-1}$, which is the total number of steps taken by a trajectory associated with $\left(N, \Theta_{\text {traj }}, x, u\right)$ in the $s$-th group of catch-up columns. Finally, for each $j \in \widetilde{V}_{\Pi, M}$ we let $v_{j} L_{n}$ be the minimal number of steps that are required to cross a column of type $\widetilde{\Theta}_{j}$. Even though it is not necessarily true that $v_{j} \leq u_{j}$ for all $j \in \widetilde{V}_{\Pi, M}$, it is true by construction that for $s \in\{1, \ldots, k\}$ we have $\bar{u}_{s} \geq v_{i_{s}}+\cdots+v_{i_{s}+r_{s}-1}=\bar{v}_{s}$. For each $s \in\{1, \ldots, k\}$ and each $t \in\left\{0, \ldots, r_{s}-1\right\}$, we define

$$
J_{i_{s}+t}=\left\{\left(\widetilde{u}_{i_{s}}+v_{i_{s}}+\cdots+v_{i_{s}+t-1}\right) L_{n},\left(\widetilde{u}_{i_{s}}+v_{i_{s}}+\cdots+v_{i_{s}+t}\right) L_{n}-1\right\},
$$

so that we partition $I_{i_{s}} \cup \cdots \cup I_{i_{s}+r_{s}}$ into

$$
\begin{aligned}
K_{s} \cup \widetilde{K}_{s} \quad \text { with } \quad K_{s} & =\left\{\widetilde{u}_{i_{s}} L_{n}, \ldots,\left(\widetilde{u}_{i_{s}}+v_{i_{s}}+\cdots+v_{i_{s}+r_{s}-1}\right) L_{n}\right\}, \\
\widetilde{K}_{s} & =\left\{\left(\widetilde{u}_{i_{s}}+v_{i_{s}}+\cdots+v_{i_{s}+r_{s}-1}\right) L_{n}+1, \ldots, \widetilde{u}_{i_{s}+r_{s}} L_{n}-1\right\},
\end{aligned}
$$

and we partition $\{1, \ldots, n\}$ into

$$
T \cup \widetilde{T} \quad \text { with } \quad \widetilde{T}=\cup_{s=1}^{k} \widetilde{K}_{s}, \quad T=\{1, \ldots, n\} \backslash \widetilde{T} .
$$

6.5.1. Step 1. In this step, we aim at replacing the Hamiltonian in the catch-up columns by an auxiliary coarse-grained version of the Hamiltonian, which simply 
assigns an energetic penalty $\frac{\beta-\alpha}{2}$ to each monomers placed in solvent $B$. To that aim, for $\chi \in\{A, B\}^{\mathbb{Z}}$ and $\pi \in \mathcal{W}_{u L}$ such that $\pi_{u L, 1}=L$, we set

$$
\widehat{H}_{u L, L}^{\chi}(\pi)=\frac{\beta-\alpha}{2} \sum_{i=1}^{u L} 1\left\{\chi_{\left(\pi_{i-1}, \pi_{i}\right)}^{L}=B\right\} .
$$

and we recall that $\chi_{\left(\pi_{i-1}, \pi_{i}\right)}^{L}$ denotes the label of the block the step $\left(\pi_{i-1}, \pi_{i}\right)$ lies in. With the help of (6.173) and recalling (4.34), we define the partition function associated with those trajectories crossing a block-column of type $\Theta=(\chi, \Xi, x)$ in $u L$ steps as

$$
\widehat{Z}_{L}(\Theta, u)=\sum_{\pi \in \mathcal{W}_{\Theta, u, L}} e^{\widehat{H}_{u L, L}^{\chi}(\pi)}
$$

and we note that $\widehat{Z}_{L}(\Theta, u)$ does not depend on the microscopic disorder $\omega$ canymore. Thus, we can set

$$
\widehat{A}_{2}=\prod_{i \in \widetilde{V}_{\Pi, M}} \widehat{Z}_{L_{n}}\left(\Theta_{i}, u_{i}\right)
$$

In the rest of this proof, we will often state results that hold uniformly on $\left(N, \Theta_{\text {traj }}, x, u\right)$ without recalling that $N \in\left\{1, \ldots, n / L_{n}\right\}, \Theta_{\text {traj }} \in \widetilde{\mathcal{D}}_{L_{n}, N}^{\infty}, x \in$ $\mathcal{X}_{\Theta_{\text {traj }}, \Omega}^{\infty, \infty}$ and $u \in \mathcal{U}_{\Theta_{\text {traj }}, x, n}^{\infty, \infty, L_{n}}$.

Our aim is to show that, for all $\varepsilon>0, M$ large enough and $\Omega \in\{A, B\}^{\mathbb{N}_{0} \times \mathbb{Z}}$, the set

$$
B_{n, M}^{1}=\left\{\omega: \widetilde{A}_{2} \leq \widehat{A}_{2} e^{\varepsilon n} \text { for all }\left(N, \Theta_{\text {traj }}, x, u\right)\right\}
$$

satisfies $\lim _{n \rightarrow \infty} \mathbb{P}_{\omega}\left(B_{n, M}^{1}\right)=1$. We consider a given $\left(N, \Theta_{\text {traj }}, x, u\right)$, and we set $\widetilde{u}=\bar{u}_{1}+\cdots+\bar{u}_{s}$. We then pick for each $i \in \widetilde{V}_{\Pi, M}$ a trajectory $\pi_{i}$ in the set $\mathcal{W}_{\Theta_{i}, u_{i}, L_{n}}$. By concatenating these trajectories, we obtain a trajectory $\widehat{\pi} \in \mathcal{W}_{\widetilde{u} L_{n}}$ satisfying $\widehat{\pi}_{\widetilde{u} L_{n}, 1}=\left(r_{1}+\cdots+r_{k}\right) L_{n}$. The difference between the Hamiltonian associated with $\widehat{\pi}$ in $\widetilde{A}_{2}$ and the one associated with $\widehat{\pi}$ in $\widehat{A}_{2}$ equals

$$
\sum_{s=1}^{k} \sum_{x=0}^{r_{s}-1} H_{u_{i_{s}+x} L_{n}, L_{n}}^{\omega_{i_{i_{s}+x}}, \Omega\left(i_{s}+x, \Pi_{i_{s}+x}+\cdot\right)}\left(\pi_{i_{s}+x}\right)-\widehat{H}_{u_{i_{s}+x} L_{n}, L_{n}}^{\Omega\left(i_{s}+x, \Pi_{i_{s}+x}+\cdot\right)}\left(\pi_{i_{s}+x}\right) .
$$

Either $\widehat{\pi}$ takes in $B$ a number of steps that is $\leq \varepsilon n /(2 \alpha)$ and the Hamiltonian difference in (6.177) is bounded above by $\varepsilon n$, or the number of steps in $B$ is larger than $\varepsilon n / 2 \alpha$. In the latter case, since $\widehat{\pi}_{\widetilde{u} L_{n}, 1}=\left(r_{1}+\cdots+r_{k}\right) L_{n}, \pi$ makes at most $\left(r_{1}+\cdots+r_{k}\right) L_{n}+\widetilde{u}$ excursions in $B$ because each such excursion requires at least one horizontal step or at least $L_{n}$ vertical steps. Therefore, by using the inequalities $\left(r_{1}+\cdots+r_{k}\right) L_{n} \leq 4 n / M$ and $\widetilde{u} \leq n / L_{n}$, we can claim that, as soon as $L_{n} \geq M$, $\widehat{\pi}$ does not perform more than $5 n / M$ excursions in $B$, and hence

$$
\begin{aligned}
\sum_{s=1}^{k} \sum_{x=0}^{r_{s}-1} H_{u_{i_{s}+x} L_{n}, L_{n}}^{\omega_{I_{i_{s}}+x}, \Omega\left(i_{s}+x, \Pi_{i_{s}+x}+\cdot\right)} & \left(\pi_{i_{s}+x}\right)-\widehat{H}_{u_{i_{s}+x} L_{n}, L_{n}}^{\Omega\left(i_{s}+x, \Pi_{i_{s}+x}+\cdot\right)}\left(\pi_{i_{s}+x}\right) \\
& \leq \max \left\{\sum_{i \in I}\left(\xi_{i}-\frac{\beta-\alpha}{2}\right): I \in \cup_{r=1}^{5 n / M} \mathcal{E}_{n, r}\right\},
\end{aligned}
$$

with $\mathcal{E}_{n, r}$ defined in (E.1) in Appendix E and $\xi_{i}=\beta 1_{\left\{\omega_{i}=A\right\}}-\alpha 1_{\left\{\omega_{i}=B\right\}}$ for $i \in \mathbb{N}$. At this point we use the definition in (E.3) and note that, for all $\omega \in \mathcal{Q}_{n, M / 5}^{\varepsilon /(2 \alpha), \varepsilon}$, the right-hand side in (6.178) is smaller than $\varepsilon n$. Consequently, for $M$ and $n$ large 
enough we have that, for all $\omega \in \mathcal{Q}_{n, M / 5}^{\varepsilon / 2 \alpha, \varepsilon}$,

$$
\frac{\widetilde{A}_{2}}{\widehat{A}_{2}} \leq e^{\varepsilon n} \quad \text { for all } \quad\left(N, \Theta_{\text {traj }}, x, u\right) .
$$

It remains to use (6.179) and (6.176) to conclude that, for $M$ and $n$ large enough, $\mathcal{Q}_{n, M / 5}^{\varepsilon / 2 \alpha, \varepsilon}$ is a subset of $B_{n, M}^{1}$. Thus, Lemma E.1 ensures that, for $M$ large enough, $\lim _{n \rightarrow \infty} P_{\omega}\left(B_{n, M}^{1}\right)=1$, which completes Step 1 .

6.5.2. Step 2. In this step we further simplify the expression of $\widehat{A}_{2}$ introduced in (6.175) by setting

$$
\widehat{A}_{3}=\prod_{i \in \widetilde{V}_{\Pi, M}} e^{\frac{\beta-\alpha}{2}} \mathfrak{N}_{B}\left(\Theta_{i}\right) L_{n},
$$

where $\mathfrak{N}_{B}\left(\Theta_{i}\right)$ is the number of $B$-blocks located in between the entrance block and the exit block that have to be crossed entirely in the vertical direction by any trajectory that crosses a block of type $\Theta_{i}$. We note that $\mathfrak{N}_{B}\left(\Theta_{i}\right)$ only depends on $\Delta \Pi_{i}$ (the mesoscopic displacement in the column) and on the disorder in the column seen from the entrance block $\Omega\left(i, \Pi_{i}+\cdot\right)$. Our aim is to show that, for $\varepsilon>0$ and for $M$ and $n$ large enough, we have for all $\Omega \in\{A, B\}^{\mathbb{N}_{0} \times \mathbb{Z}}$ that $\widehat{A}_{2}<\widehat{A}_{3} e^{\varepsilon n}$ uniformly in $(N, \Theta, x, u)$.

For a given $(N, \Theta, x, u)$ we pick, for each $i \in \widetilde{V}_{\Pi, M}$, a trajectory $\pi_{i} \in \mathcal{W}_{\Theta_{i}, u_{i}, L_{n}}$. We recall that $\beta-\alpha \leq 0$, since $(\alpha, \beta) \in$ CONE. In $\widehat{A}_{2}$ the Hamiltonian associated with $\left(\pi_{i}\right)_{i \in \widetilde{V}_{\Pi, M}}$ is bounded above by

$$
\sum_{i \in \widetilde{V}_{\Pi, M}} \widehat{H}_{u_{i} L_{n}, L_{n}}^{\Omega\left(i, \Pi_{i}+\cdot\right)}\left(\pi_{i}\right) \leq \frac{\beta-\alpha}{2} L_{n} \sum_{i \in \widetilde{V}_{\Pi, M}} \mathfrak{N}_{B}\left(\Theta_{i}\right)
$$

because, for each $i \in \widetilde{V}_{\Pi, M}, \pi_{i}$ must cross vertically at least $\mathfrak{N}_{B}\left(\Theta_{i}\right)$ blocks of type $B$. In the right-hand side of (6.181), we recognise the exponential factor in (6.180), and therefore this step will be complete once we control the entropy carried by those pieces of trajectories that cross the columns indexed in $\widetilde{V}_{\Pi, M}$. To that aim, we recall that $\widetilde{u}=\bar{u}_{1}+\cdots+\bar{u}_{s}$ and we note that, by concatenating the paths $\left(\pi_{i}\right)_{i \in \widetilde{V}_{\Pi, M}}$, we obtain a trajectory $\widehat{\pi} \in \mathcal{W}_{\widetilde{u} L_{n}}$ satisfying $\pi_{\widetilde{u} L_{n}, 1}=\left(r_{1}+\cdots+r_{k}\right) L_{n}$. Thus,

$$
\prod_{i \in \widetilde{V}_{\Pi, M}}\left|\mathcal{W}_{\Theta_{i}, u_{i}, L_{n}}\right| \leq\left|\left\{\pi \in \mathcal{W}_{\widetilde{u} L_{n}}: \pi_{\widetilde{u} L_{n}, 1}=\left(r_{1}+\cdots+r_{k}\right) L_{n}\right\}\right|,
$$

and either $\widetilde{u} \leq \varepsilon n / \log (3) L_{n}$ and the right-hand side in (6.182) is smaller than $e^{\varepsilon n}$, or $\widetilde{u} \geq \varepsilon n / \log (3) L_{n}$ and the crude bound $r_{1}+\cdots+r_{k} \leq 4 n / M L_{n}$ allows us to write $\widetilde{u} /\left(r_{1}+\ldots, r_{k}\right) \geq M \varepsilon /(4 \log (3))$, and we can use Lemma A.2 in Appendix A to assert that, for $M$ large enough, the right-hand side of (6.182) is bounded above by $e^{\varepsilon n}$.

6.5.3. Step 3. In this step, we link each mesoscopic strategy $\Theta_{\text {traj }}$ to its auxiliary counterpart $\widetilde{\Theta}_{\text {traj }}$ by replacing $\widehat{A}_{3}$ in $(6.180)$ by

$$
\widehat{A}_{4}=\prod_{i \in \widetilde{V}_{\Pi, M}} e^{\frac{\beta-\alpha}{2} \mathfrak{N}_{B}\left(\widetilde{\Theta}_{i}\right) L_{n}} .
$$


As in the previous step, $\mathfrak{N}_{B}\left(\widetilde{\Theta}_{i}\right)$ is the number of $B$-blocks located in between the entrance and the exit blocks that have to be crossed entirely in the vertical direction by any trajectory crossing the $\widetilde{\Theta}_{i}$ column. Our aim is to show that for all $\varepsilon>0$ there exists an $M \in \mathbb{N}$ such that, for $\mathbb{P}$ - a.e. $\Omega$ and $n$ is large enough, that $\widehat{A}_{3} \leq \widehat{A}_{4} e^{\varepsilon n}$ for all $(N, \Theta, x, u)$. To that aim, it suffices to prove that for all $\varepsilon>0$ there exists an $M \in \mathbb{N}$ such that, for $\mathbb{P}$ - a.e. $\Omega$ and $n$ large enough, that

$$
\sum_{i \in \widetilde{V}_{\Pi, M}} \mathfrak{N}_{B}\left(\Theta_{i}\right) \geq \sum_{i \in \widetilde{V}_{\Pi, M}} \mathfrak{N}_{B}\left(\widetilde{\Theta}_{i}\right)-\frac{\varepsilon n}{L_{n}}, \text { for all }\left(N, \Theta_{\text {traj }}, x, u\right) .
$$

We set

$$
\begin{aligned}
R_{n, M}=\left\{\Omega \in\{A, B\}^{\mathbb{N}_{0} \times \mathbb{Z}}:\right. & \exists N \in\left\{1, \ldots, \frac{n}{L_{n}}\right\}, \exists \Theta_{\text {traj }} \in \widetilde{\mathcal{D}}_{L_{n}, N}^{\infty}, \\
& \text { and } \left.\sum_{i \in \widetilde{V}_{u, M}} \mathfrak{N}_{B}\left(\Theta_{i}\right) \leq \sum_{i \in \widetilde{V}_{u, M}} \mathfrak{N}_{B}\left(\widetilde{\Theta}_{i}\right)-\varepsilon \frac{n}{L_{n}}\right\}
\end{aligned}
$$

and we aim at showing that, for $M$ large enough, $\sum_{n \geq 1} \mathbb{P}_{\Omega}\left(R_{n, M} \neq \emptyset\right)<\infty$.

We need to simplify the expression for $R_{n, M}$. As explained earlier, for each $\left(N, \Theta_{\text {traj }}, x, u\right)$, the location of the catch-up columns $\widetilde{V}_{\Pi, M}$ only depends on $\Pi=$ $\left(\Pi_{i}\right)_{i=0}^{N}$ and the subsequence $\left(\mathfrak{N}_{B}\left(\Theta_{i}\right)\right)_{i \in \widetilde{V}_{\Pi, M}}$ only depends on $\Omega$ and $\left(\Pi_{i}, \Pi_{i+1}\right)_{i \in \widetilde{V}_{\Pi, M}}$. Moreover, in the catch-up columns, the associated mesoscopic strategy of displacement $\left(\widetilde{\Pi}_{i}, \widetilde{\Pi}_{i+1}\right)_{i \in \widetilde{V}_{\Pi, M}}$ is completely determined by $\left(\Pi_{i}, \Pi_{i+1}\right)_{i \in \widetilde{V}_{\Pi, M}}$ and $\left(\mathfrak{N}_{B}\left(\widetilde{\Theta}_{i}\right)\right)_{i \in \widetilde{V}_{\Pi, M}}$ only depends on $\Omega$ and $\left(\widetilde{\Pi}_{i}, \widetilde{\Pi}_{i+1}\right)_{i \in \widetilde{V}_{\Pi, M}}$. As a consequence, for all $i \in \widetilde{V}_{\Pi, M}$ we can rewrite $\mathfrak{N}_{B}\left(\Theta_{i}\right)$ and $\mathfrak{N}_{B}\left(\widetilde{\Theta}_{i}\right)$ as $\mathfrak{N}_{B}\left(\Omega\left(i, \Pi_{i}+\cdot\right), \Delta \Pi_{i}\right)$ and $\mathfrak{N}_{B}\left(\Omega\left(i, \widetilde{\Pi}_{i}+\cdot\right), \Delta \widetilde{\Pi}_{i}\right)$, respectively, and we obtain

$$
R_{n, M} \subset \bigcup_{N=1}^{n / L_{n}} \bigcup_{k=1}^{4 n / M L_{n}} \bigcup_{V \subset\{0, \ldots, N-1\}:|V|=k} \bigcup_{\bar{Y} \in \mathfrak{V}_{N, V}} \bar{R}(N, k, V, \bar{Y}),
$$

where

$$
\begin{aligned}
& \mathfrak{V}_{N, V}=\left\{\bar{Y}:=\left(Y_{i}^{0}, Y_{i}^{1}\right)_{i \in V} \in\left(\mathbb{Z}^{2}\right)^{V}: Y_{i}^{1}=Y_{i+1}^{0} \text { if }(i, i+1) \in V^{2}\right. \text { and } \\
& \left.\exists \Pi \in\{0\} \times \mathbb{Z}^{N}: \sum_{i=0}^{N-1}\left|\Delta \Pi_{i}\right| \leq \frac{n}{L_{n}}, \widetilde{V}_{\Pi, M}=V,\left(\Pi_{i}, \Pi_{i+1}\right)_{i \in V}=\left(Y_{i}^{0}, Y_{i}^{1}\right)_{i \in V}\right\},
\end{aligned}
$$

where

$$
\begin{aligned}
\bar{R}(N, k, V, \bar{Y})=\{ & \Omega \in\{A, B\}^{\mathbb{N}_{0} \times \mathbb{Z}}: \\
& \left.\sum_{i \in V} \mathfrak{N}_{B}\left(\Omega\left(i, Y_{i}+\cdot\right), \Delta Y_{i}\right) \leq \sum_{i \in V} \mathfrak{N}_{B}\left(\Omega\left(i, \tilde{Y}_{i}+\cdot\right), \Delta \tilde{Y}_{i}\right)-\varepsilon \frac{n}{L_{n}}\right\}
\end{aligned}
$$

and where, with each $\bar{Y} \in \mathfrak{V}_{N, V}$, we associate $\widetilde{Y}=\left(\widetilde{\Pi}_{i}, \widetilde{\Pi}_{i+1}\right)_{i \in V}$ with $\widetilde{\Pi}$ the mesoscopic displacement strategy associated with the $\Pi$, which in (6.187) guarantees that $\bar{Y} \in \mathfrak{V}_{N, V}$.

For $N \in\left\{1, \ldots, n / L_{n}\right\}, k \in\left\{1, \ldots, 4 n / M L_{n}\right\}, V \subset\{0, \ldots, N-1\}:|V|=k$ and $\bar{Y} \in \mathfrak{V}_{N, V}$, we let $\mathfrak{N}(\bar{Y})$ be the number of blocks that have to be crossed entirely in 
the vertical direction in the catch-up columns (i.e., those columns indexed in $V$ ). By construction, we note that $\mathfrak{N}(\bar{Y}) \geq \mathfrak{N}(\widetilde{Y})$, so that the number of blocks that have to be crossed vertically in the catch-up columns for the mesoscopic strategy of displacement $\bar{Y}$ is not smaller than its counterpart for the auxiliary mesoscopic strategy of displacement $\widetilde{Y}$. We then note that $\Omega \in R_{n, M}$ necessarily implies that there exists $N, k, V, \bar{Y}$ such that $\mathfrak{N}(\widetilde{Y}) \geq \varepsilon n / L_{n}$, and therefore $\mathfrak{N}(\bar{Y}) \geq \varepsilon n / L_{n}$ (since it is always the case that $\mathfrak{N}(\bar{Y}) \geq \mathfrak{N}(\widetilde{Y}) \geq \sum_{i \in V} \mathfrak{N}_{B}\left(\Omega\left(i, \widetilde{Y}_{i}+\cdot\right), \Delta \widetilde{Y}_{i}\right)$ ). As a consequence, we can bound $\mathbb{P}_{\Omega}\left(R_{n, M} \neq \emptyset\right)$ as follows:

$$
\mathbb{P}_{\Omega}\left(R_{n, M} \neq \emptyset\right) \leq \sum_{N=1}^{n / L_{n}} \sum_{k=1}^{4 n / M L_{n}} \sum_{\substack{V \subset\{0, \ldots, N-1\}: \\|V|=k}} \sum_{\substack{\bar{Y} \in \mathfrak{V}_{N}, V \\ \mathfrak{N}(\bar{Y}) \geq \varepsilon n / L_{n}}} \mathbb{P}_{\Omega}(\bar{R}(N, k, V, \bar{Y})) .
$$

By a standard application of Cramer's Theorem we obtain that the probability under the sum of the right-hand side in (6.189) is uniformly bounded by $e^{-c_{\varepsilon} n / L_{n}}$ with $c_{\varepsilon}>0$. At this stage we note that, uniformly in $N$ and $k$, we can bound $|V \subset\{0, \ldots, N-1\}:| V|=k|$ from above by $\left(\begin{array}{c}n / L_{n} \\ 4 n / M L_{n}\end{array}\right)$, which for $M$ large enough has an exponential growth rate that is smaller than $c_{\varepsilon}$. Moreover, uniformly in $N, k, V$

$$
\left|\mathfrak{V}_{N, V}\right| \leq 2^{k}\left(\begin{array}{c}
n / L_{n} \\
2 k
\end{array}\right)\left(\frac{n}{k L_{n}}\right)^{k} \leq 2^{\frac{4 n}{M L_{n}}}\left(\begin{array}{c}
n / L_{n} \\
8 n / M L_{n}
\end{array}\right)\left(\frac{M}{8}\right)^{\frac{8 n}{M L_{n}}} .
$$

The upper bound in (6.190) can be understood as follows. First, in each catchup columns we have to choose the length of the mesoscopic displacement and this gives rise to the term $\left(\begin{array}{c}n / L_{n} \\ 2 k\end{array}\right)$, since the sum of all mesoscopic increments is bounded above by $n / L_{n}$. Next, we have to choose the sign of these $k$ increments and this gives a factor $2^{k}$. Finally, in each catch-up columns we have to choose the height of the entrance block $\left(\Pi_{i}\right)$. Once again, the fact that the sum of all mesoscopic displacement is smaller than $n / L_{n}$ tells us that the difference between the height of the exit block of a given catch-up column and the height of the entrance block of the following catch-up column is bounded by the sum of the absolute value of the mesoscopic increments that have been made in between these two columns. But once again, since the sum of these mesoscopic displacements in absolute value is smaller than $n / L_{n}$ the number of choices for the heights of all entrance blocks in catch-up columns is bounded above by $\left(n / k L_{n}\right)^{k}$. This completes the proof because when $M$ is chosen large enough the exponential growth rate in the right-hand side of (6.190) is smaller than $c_{\varepsilon}$.

6.5.4. Step 4. In this step, we recall the coarse-grained version of the Hamiltonian defined in (6.173) and we use it to introduce, in the catch-up columns, those trajectories moving according to the auxiliary mesoscopic strategy, i.e., for $\left(N, \Theta_{\text {traj }}, x, u\right)$ we set

$$
\widehat{A}_{5}=\prod_{i \in \widetilde{V}_{\Pi, M}} \widehat{Z}_{L_{n}}\left(\widetilde{\Theta}_{i}, v_{i}\right)
$$

with $\widetilde{\Theta}_{i}=\left(\Omega\left(i, \widetilde{\Pi}_{i}+\cdot\right), \widetilde{\Xi}_{i}, 1\right)$. Our aim is to prove that, for $\varepsilon>0$ and $M$ large enough, we have for all $\Omega \in\{A, B\}^{\mathbb{N}_{0} \times \mathbb{Z}}$ and all $n \in \mathbb{N}$ that $\widehat{A}_{4} \leq \widehat{A}_{5} e^{\varepsilon n}$ uniformly in $(N, \Theta, x, u)$. 
For each $i \in \widetilde{V}_{\Pi, M}$, we pick $\pi_{i} \in \mathcal{W}_{\widetilde{\Theta}_{i}, v_{i}, L_{n}}$. Since $v_{i} L_{n}$ is the minimal number of steps required to cross the column indexed by $i$, and since $\mathfrak{N}_{B}\left(\widetilde{\Theta}_{i}\right)$ is the number of $B$-blocks that have to be crossed vertically by any trajectory crossing a block column of type $\widetilde{\Theta}_{i}$, we can assert that the number of steps performed by $\pi_{i}$ in the $B$-blocks belongs to $\left\{\mathfrak{N}_{B}\left(\widetilde{\Theta}_{i}\right) L_{n}, \ldots, \mathfrak{N}_{B}\left(\widetilde{\Theta}_{i}\right) L_{n}+3 L_{n}\right\}$. Therefore, recalling the definition (6.173) and the crude bound $r_{1}+\cdots+r_{k} \leq 4 n / M L_{n}$, we can assert that, for all $\left(N, \Theta_{\text {traj }}, x, u\right)$ and all $\left(\pi_{i}\right)_{i \in \tilde{V}_{\Pi, M}} \in \bigotimes_{i \in \tilde{V}_{\Pi, M}} \mathcal{W}_{\widetilde{\Theta}_{i}, v_{i}, L_{n}}$,

$$
\sum_{s=1}^{k} \sum_{x=0}^{r_{s}-1} \widehat{H}_{v_{i_{s}+x} L_{n}, L_{n}}^{\Omega\left(i_{s}+x, \widetilde{\Pi}_{i_{s}+x}+\cdot\right)}\left(\pi_{i_{s}+x}\right) \geq \frac{\beta-\alpha}{2} \sum_{i \in \widetilde{V}_{\Pi, M}} \mathfrak{N}_{B}\left(\widetilde{\Theta}_{i}\right) L_{n}-12 \frac{\alpha n}{M L_{n}} .
$$

Thus, it suffices to choose $M$ so large that $12 \alpha / M \leq \varepsilon$ to complete the proof of the step.

6.5.5. Step 5. In this step we replace, for each of the pieces of trajectories crossing the catch-up column, the coarse-grained Hamiltonian $\widehat{H}$ by the original Hamiltonian. Thus, we set

$$
\widehat{A}_{6}=\prod_{i \in \widetilde{V}_{\Pi, M}} Z_{L_{n}}^{\omega_{J_{i}}}\left(\widetilde{\Theta}_{i}, v_{i}\right) .
$$

Our aim is to show that, for $\varepsilon>0$ and $M$ large enough, we have for all $\Omega \in$ $\{A, B\}^{\mathbb{N}_{0} \times \mathbb{Z}}$ that

$$
B_{n, M}^{2}=\left\{\omega: \widehat{A}_{5} \leq \widehat{A}_{6} e^{\varepsilon n} \text { for all }\left(N, \Theta_{\text {traj }}, x, u\right)\right\}
$$

satisfies $\mathbb{P}_{\omega}\left(B_{n, M}^{2}\right) \rightarrow 1$ as $n \rightarrow \infty$. We will not give the details of the proof, because it is completely similar to that of Step 1 . The only difference is that we replace $\widetilde{u}=\bar{u}_{1}+\cdots+\bar{u}_{r}$ by $\widetilde{v}=\bar{v}_{1}+\cdots+\bar{v}_{r}$.

6.5.6. Step 6. Let $\left(\widetilde{w}_{i}\right)_{i \in \mathbb{N}}$ be an i.i.d. sequence of Bernouilli trials, independent of $\omega, \Omega$. For $\left(N, \Theta_{\text {traj }}, x, u\right)$ we set $\widehat{u}=\sum_{s=1}^{k} \bar{u}_{s}-\bar{v}_{s}$. By changing the $u_{i}$ into $v_{i}$ in those catch-up columns, we remove $\widehat{u} L_{n}$ steps from the trajectories associated with $\left(N, \Theta_{\text {traj }}, x, u\right)$, so that they have become trajectories associated with $\left(N, \widetilde{\Theta}_{\text {traj }}, \widetilde{x}, v\right)$. In this step, we will concatenate the trajectories associated with $\left(N, \widetilde{\Theta}_{\text {traj }}, \widetilde{x}, v\right)$ with an $\widehat{u} L_{n}$-step trajectory to recover a trajectory that belongs to $\mathcal{W}_{n, M}$.

Therefore, our aim is to show that for all $\varepsilon>0$ there exists an $M \in \mathbb{N}$ such that, for $\mathbb{P}-$ a.e. $\Omega$ and for all $\omega, \widetilde{\omega} \in\{A, B\}^{\mathbb{N}}$, we have for $n$ large enough that $\widetilde{A}_{2} \widehat{A}_{6} \leq e^{\varepsilon n} Z_{n, L_{n}}^{\left(\omega_{J}, \widetilde{\omega}\right), \Omega}(M)$ for all $(N, \Theta, x, u)$. The proof is completely similar to that of Step 2 in the proof of Proposition 6.3 (see Section 6.3.2). For this reason, we will not repeat the details.

6.5.7. Step \%. In this step, we average over the microscopic disorders $\omega, \widetilde{\omega}$. We recall (6.176) and (6.144), and we set $B_{n, M}=B_{n, M}^{1} \cap B_{n, M}^{2}$. With the help of Steps 1-6 above we can state that for every $\varepsilon>0$ there exists an $M \in \mathbb{N}$ such that, for $\mathbb{P}-$ a.e. $\Omega$ and for $\omega \in B_{n, M}$ and $\widetilde{\omega} \in\{A, B\}^{\mathbb{N}}$, we have (recall (6.169))

$$
A_{1}=A_{2} \widetilde{A}_{2} \leq e^{6 \varepsilon n} Z_{n, L_{n}}^{\left(\omega_{J}, \widetilde{\omega}\right), \Omega}(M) \text { for all }(N, \Theta, x, u) .
$$


Next, we recall (4.11) and (6.138), and we use (6.195) to state that for all $\varepsilon>0$ there exists an $M \in \mathbb{N}$ such that, for $\mathbb{P}$ - a.e. $\Omega$ and $n$ large enough and for $\omega \in B_{n, M}$ and $\widetilde{\omega} \in\{A, B\}^{\mathbb{N}}$,

$$
Z_{n, L_{n}}^{\omega, \Omega} \leq e^{6 \varepsilon n} \sum_{N=1}^{n / L_{n}} \sum_{\Theta_{\text {traj }} \in \widetilde{\mathcal{D}}_{L_{n}, N}^{\infty}} \sum_{x \in \mathcal{X}_{\Theta_{\text {traj }}^{\infty, \infty}}^{\infty}} \sum_{u \in \mathcal{U}_{\Theta_{\text {traj }}^{\infty, \infty, n}, n}} Z_{n, L_{n}}^{\left(\omega_{J}, \widetilde{\omega}\right), \Omega}(M) .
$$

We use (D.3) to claim that there exists $C_{1}, C_{2}>0$ such that, for all for all $\Omega$, all $n \in \mathbb{N}$, all $M \in \mathbb{N}$ and all $J$,

$$
\mathbb{P}_{\omega, \widetilde{\omega}}\left(\left|\frac{1}{n} \log Z_{n, L_{n}}^{\left(\omega_{J}, \widetilde{\omega}\right), \Omega}(M)-f_{n}^{\Omega}(M)\right| \geq \varepsilon\right) \leq C_{1} e^{-C_{2} \varepsilon^{2} n} .
$$

We set also

$$
D_{n, M}=\bigcap_{\left(N, \Theta_{\text {traj }}, x, u\right)}\left\{\left|\frac{1}{n} \log Z_{n, L_{n}}^{\left(\omega_{J}, \widetilde{\omega}\right), \Omega}(M)-f_{n}^{\Omega}(M)\right| \leq \varepsilon\right\},
$$

recall the definition of $c_{n}$ in (6.57) (with $\left.M=m=\infty\right)$ ), and use (6.197) and the fact that $c_{n}$ grows subexponentially in $n$, to obtain $\lim _{n \rightarrow \infty} \mathbb{P}_{\omega, \widetilde{\omega}}\left(D_{n, M}^{c}\right)=0$. For all $(\omega, \widetilde{\omega})$ satisfying $\omega \in B_{n, M}$ and $(\omega, \widetilde{\omega}) \in D_{n, M}$, we can rewrite (6.196) as

$$
Z_{n, L_{n}}^{\omega, \Omega} \leq c_{n} e^{n f_{n}^{\Omega}(M)+7 \varepsilon n} .
$$

Consequently, recalling (4.37), for $M$ large enough we have

$f_{n}^{\Omega}(\alpha, \beta) \leq \mathbb{P}\left(B_{n, M}^{c} \cup D_{n, M}^{c}\right) C_{\mathrm{uf}}(\alpha)+\frac{\log c_{n}}{n}+\frac{1}{n} \mathbb{E}\left(1_{\left\{B_{n, M} \cup D_{n, M}\right\}}\left(n f_{n}^{\Omega}(M)+7 \varepsilon n\right)\right)$.

Since $\mathbb{P}\left(B_{n, M}^{c} \cup D_{n, M}^{c}\right)$ and $\lim _{n \rightarrow \infty}\left(\log c_{n}\right) / n=0$, the proof of Proposition 6.5 is complete.

\section{Proof of Theorem 2.1: slope-based variational formula}

We are now ready to show how the variational formula in (6.2) can be transformed into the variational formula in (2.14). We recall that, by the definition of $\overline{\mathcal{R}}_{p}$ in (4.63), the variational formula in (2.14) can also be written as

$$
f(\alpha, \beta ; p)=\sup _{M \geq 1} \sup _{\bar{\rho} \in \overline{\mathcal{R}}_{p, M}} \sup _{v \in \overline{\mathcal{B}}} \frac{\bar{N}(\bar{\rho}, v)}{\bar{D}(\bar{\rho}, v)}
$$

Let $\mathcal{F}_{\overline{\mathcal{V}}_{M}}$ and $\overline{\mathcal{F}}$ be the counterparts of $\mathcal{B}_{\overline{\mathcal{V}}_{M}}$ and $\overline{\mathcal{B}}$ for Borel functions instead of continuous functions, i.e.,

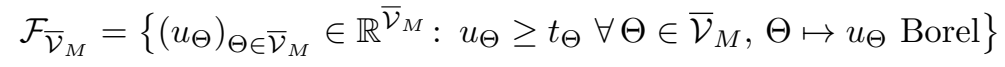

and

$$
\overline{\mathcal{F}}=\left\{v=\left(v_{A}, v_{B}, v_{\mathcal{I}}\right) \in \overline{\mathcal{D}} \times \overline{\mathcal{D}} \times[1, \infty)\right\}
$$

where

$$
\overline{\mathcal{D}}=\left\{l \mapsto v_{l} \text { on }[0, \infty) \text { Lebesgue measurable with } v_{l} \geq 1+l \quad \forall l \geq 0\right\} .
$$

The proof of Theorem 2.1 is divided into 4 steps, organized as Sections 7.1-7.4. In Step 1 we show that the supremum over $\mathcal{B}_{\overline{\mathcal{V}}_{M}}$ in (6.2) may be extended to $\mathcal{F}_{\overline{\mathcal{V}}_{M}}$, 
i.e.,

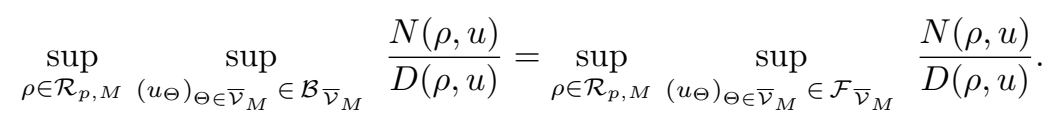

In Step 2 we show that the supremum over $\overline{\mathcal{B}}$ in (2.14) may be extended to $\overline{\mathcal{F}}$, i.e.,

$$
\sup _{\bar{\rho} \in \overline{\mathcal{R}}_{p, M}} \sup _{v \in \overline{\mathcal{B}}} \frac{\bar{N}(\bar{\rho}, v)}{\bar{D}(\bar{\rho}, v)}=\sup _{\bar{\rho} \in \overline{\mathcal{R}}_{p, M}} \sup _{v \in \overline{\mathcal{F}}} \frac{\bar{N}(\bar{\rho}, v)}{\bar{D}(\bar{\rho}, v)} .
$$

Then, the proof of Theorem 2.1 is achieved with the help of Steps 3 and 4 which, combined with Theorem 6.1, allow us to show

$$
\begin{aligned}
& f(\alpha, \beta ; p) \geq \sup _{M \geq 1} \sup _{\bar{\rho} \in \overline{\mathcal{R}}_{p, M}} \sup _{v \in \overline{\mathcal{F}}} \frac{\bar{N}(\bar{\rho}, v)}{\bar{D}(\bar{\rho}, v)}, \\
& f(\alpha, \beta ; p) \leq \sup _{M \geq 1} \sup _{\bar{\rho} \in \overline{\mathcal{R}}_{p, M}} \sup _{v \in \overline{\mathcal{F}}} \frac{\bar{N}(\bar{\rho}, v)}{\bar{D}(\bar{\rho}, v)} .
\end{aligned}
$$

Along the way we will need a few technical facts, which are collected in Appendices $\mathrm{C}-\mathrm{G}$.

7.1. Step 1: extension of the variational formula. For $c \in(0, \infty)$, let $u(c)=$ $\left(u_{\Theta}(c)\right)_{\Theta \in \overline{\mathcal{V}}_{M}}$ be the counterpart of the function $v(c)$ introduced in (3.8-3.10). For $\Theta \in \overline{\mathcal{V}}_{M}$ and $c \in(0, \infty)$, set

$$
u_{\Theta}(c)= \begin{cases}t_{\Theta} & \text { if } \partial_{u}^{+}(u \psi(\Theta, u))\left(t_{\Theta}\right) \leq c \\ z & \text { otherwise, with } z \text { such that } \\ & \partial_{u}^{-}(u \psi(\Theta, u))(z) \geq c \geq \partial_{u}^{+}(u \psi(\Theta, u))(z),\end{cases}
$$

where $z$ exists and is finite by Lemma C.7 in Appendix C, and is unique by the strict concavity of $u \rightarrow u \psi(\Theta, u)$ for $\Theta \in \overline{\mathcal{V}}_{M}$ (see Lemma C.4 in Appendix C). The fine properties of $\Theta \mapsto u_{\Theta}(c)$ are given in Lemma B.4 in Appendix B.

For $(\alpha, \beta) \in$ CONE and $\rho \in \mathcal{M}_{1}\left(\overline{\mathcal{V}}_{M}\right)$ such that $\int_{\overline{\mathcal{V}}_{M}} t_{\Theta} \rho(d \Theta)<\infty$, set

$$
g(\rho ; \alpha, \beta)=\sup _{u \in \mathcal{F}_{\overline{\mathcal{V}}_{M}}} \frac{N(\rho, u)}{D(\rho, u)},
$$

with the convention that $N(\rho, u) / D(\rho, u)=-\infty$ when $D(\rho, u)=\infty$. The equality in (7.5) is a straightforward consequence of the following lemma.

Lemma 7.1. For $(\alpha, \beta) \in \operatorname{CONE}$ and $\rho \in \mathcal{M}_{1}\left(\overline{\mathcal{V}}_{M}\right)$ such that $g(\rho ; \alpha, \beta)>0$,

$$
g(\rho ; \alpha, \beta)=\frac{N(\rho, \bar{u})}{D(\rho, \bar{u})} \quad \text { with } \bar{u}=u(g(\rho ; \alpha, \beta)) .
$$

Moreover, $u=\bar{u}$ for $\rho$-a.e. $\Theta \in \overline{\mathcal{V}}_{M}$ for all $u \in \mathcal{F}_{\overline{\mathcal{V}}_{M}}$ satisfying $g(\rho ; \alpha, \beta)=\frac{N(\rho, u)}{D(\rho, u)}$.

Proof: The following lemma will be needed in the proof.

Lemma 7.2. For $(\alpha, \beta) \in \mathrm{CONE}$ and $\varepsilon>0$ there exists a $t_{\varepsilon}>0$ such that, for all $\rho \in \mathcal{M}_{1}\left(\overline{\mathcal{V}}_{M}\right)$ and all $u \in \mathcal{F}_{\overline{\mathcal{V}}_{M}}$ satisfying $D(\rho, u) \in\left(t_{\varepsilon}, \infty\right)$,

$$
\frac{N(\rho, u)}{D(\rho, u)} \leq \varepsilon
$$


Proof: Pick $\varepsilon>0$. By Lemma C.6, there exists a $C_{\varepsilon}>0$ such that $\psi(\Theta, u) \leq \varepsilon / 2$ for $\Theta \in \overline{\mathcal{V}}_{M}$ and $u \geq \max \left\{C_{\varepsilon}, t_{\Theta}\right\}$. For $R \in(0, \infty)$, set $B^{-}(R)=\left\{\Theta \in \overline{\mathcal{V}}_{M}: u_{\Theta} \leq\right.$ $R\}$ and $B^{+}(R)=\left\{\Theta \in \overline{\mathcal{V}}_{M}: u_{\Theta}>R\right\}$, and write

$$
\frac{N(\rho, u)}{D(\rho, u)}=\frac{\int_{B^{-}\left(C_{\varepsilon}\right)} u_{\Theta} \psi\left(\Theta, u_{\Theta}\right) \rho(d \Theta)}{D(\rho, u)}+\frac{\int_{B^{+}\left(C_{\varepsilon}\right)} u_{\Theta} \psi\left(\Theta, u_{\Theta}\right) \rho(d \Theta)}{D(\rho, u)} .
$$

By the definition of $C_{\varepsilon}$ and since $u_{\Theta} \geq t_{\Theta}$ for all $\Theta \in \overline{\mathcal{V}}_{M}$, we can bound the second term in the right-hand side of (7.13) by $\varepsilon / 2>0$. The first term in the right-hand side of (7.13) in turn can be bounded from above by $C_{\varepsilon} C_{\mathrm{uf}}(\alpha) / D(\rho, u)$ (recall (4.37)). Consequently, it suffices to choose $t_{\varepsilon}=2 C_{\varepsilon} C_{\mathrm{uf}}(\alpha) / \varepsilon$ to complete the proof.

We resume the proof of Lemma 7.1. By assumption, we know that $g(\rho)>$ 0 , which entails that $\int_{\overline{\mathcal{V}}_{M}} t_{\Theta} \rho(d \Theta)<\infty$. Thus, Lemma B.4(iv) tells us that $D(\rho, u(c))<\infty$ for all $c>0$. We argue by contradiction. Suppose that $\frac{N(\rho, \bar{u})}{D(\rho, \bar{u})}<$ $g(\rho)$, and pick $u \in \mathcal{F}_{\overline{\mathcal{V}}_{M}}$ such that $D(\rho, u)<\infty$. Write

$$
\frac{N(\rho, u)}{D(\rho, u)}=\frac{N(\rho, \bar{u})+[N(\rho, u)-N(\rho, \bar{u})]}{D(\rho, \bar{u})+[D(\rho, u)-D(\rho, \bar{u})]}
$$

where

$$
N(\rho, u)-N(\rho, \bar{u})=\int_{\overline{\mathcal{V}}_{M}} u_{\Theta} \psi\left(\Theta, u_{\Theta}\right)-\bar{u}_{\Theta} \psi\left(\Theta, \bar{u}_{\Theta}\right) \rho(d \Theta) .
$$

The strict concavity of $u \mapsto u \psi(\Theta, u)$ on $\left[t_{\Theta}, \infty\right)$ for every $\Theta \in \overline{\mathcal{V}}_{M}$, together with the definition of $\bar{u}$ in (7.11), allows us to estimate

$$
N(\rho, u)-N(\rho, \bar{u}) \leq g(\rho) \int_{\overline{\mathcal{V}}_{M}}\left(u_{\Theta}-\bar{u}_{\Theta}\right) \rho(d \Theta) .
$$

Consequently, (7.14) becomes

$$
\frac{N(\rho, u)}{D(\rho, u)} \leq \frac{N(\rho, \bar{u})+g(\rho)[\bar{D}(\rho, u)-\bar{D}(\rho, \bar{u})]}{D(\rho, \bar{u})+[D(\rho, u)-D(\rho, \bar{u})]} .
$$

Define $G=x \mapsto[N(\rho, \bar{u})+g(\bar{\rho}) x] /[D(\rho, \bar{u})+x]$ on $(-D(\rho, \bar{u}), \infty)$. Note that $N(\rho, \bar{u}) / D(\rho, \bar{u})<g(\rho)$ implies that $G$ is strictly increasing with $\lim _{x \rightarrow \infty} G(x)=$ $g(\rho)$. Use Lemma 7.2 to assert that $\mathbb{N}(\rho, u) / D(\rho, u) \leq \frac{1}{2} g(\rho)$ when $D(\rho, u) \geq t_{\frac{1}{2} g(\rho)}$. But then, for all $u$ satisfying $D(\rho, u) \leq t_{\frac{g(\rho)}{2}},(7.17)$ gives

$$
\frac{N(\rho, u)}{D(\rho, u)} \leq G\left(t_{\frac{g(\rho)}{2}}-D(\rho, \bar{u})\right)<g(\rho)
$$

Consequently,

$$
\sup _{u \in \mathcal{F}_{\overline{\mathcal{V}}_{M}}} \frac{N(\rho, u)}{D(\rho, u)} \leq \max \left\{\frac{g(\rho)}{2}, G\left(t_{\frac{g(\rho)}{2}}-D(\rho, \bar{u})\right)\right\}<g(\rho),
$$

which is a contradiction, and so $g(\rho)=N(\rho, \bar{u}) / D(\rho, \bar{u})$.

It remains to prove that if $u \in \mathcal{F}_{\overline{\mathcal{V}}_{M}}$ satisfies $g(\rho)=N(\rho, u) / D(\rho, u)$, then $u=\bar{u}$ for $\rho$-a.e. $\Theta \in \overline{\mathcal{V}}_{M}$. We proceed again by contradiction, i.e., we suppose that a such $u$ is not equal to $\bar{u}$ for $\rho$-a.e. $\Theta \in \overline{\mathcal{V}}_{M}$. In this case, both inequalities in (7.16) and (7.17) are strict, which immediately yields that $\frac{N(\rho, u)}{D(\rho, u)}<g(\rho)$. 
7.2. Step 2: extension of the reduced variational formula. Recall (3.8-3.10) and, for $(\alpha, \beta) \in \mathrm{CONE}$ and $\bar{\rho} \in \mathcal{M}_{1}\left(\mathbb{R}_{+} \cup \mathbb{R}_{+} \cup\{\mathcal{I}\}\right)$ such that $\int_{0}^{\infty}(1+l)\left[\bar{\rho}_{A}+\bar{\rho}_{B}\right](d l)<\infty$, set

$$
h(\bar{\rho} ; \alpha, \beta)=\sup _{v \in \overline{\mathcal{F}}} \frac{\bar{N}(\bar{\rho}, v)}{\bar{D}(\bar{\rho}, v)} .
$$

Recall (3.8-3.10). The equality in (7.6) is a straightforward consequence of the following lemma.

Lemma 7.3. For $(\alpha, \beta) \in \mathrm{CONE}$ and $\bar{\rho} \in \mathcal{M}_{1}\left(\mathbb{R}_{+} \cup \mathbb{R}_{+} \cup\{\mathcal{I}\}\right)$ such that $h(\bar{\rho} ; \alpha, \beta)>$ 0 ,

$$
h(\bar{\rho} ; \alpha, \beta)=\frac{\bar{N}(\bar{\rho}, \bar{v})}{\bar{D}(\bar{\rho}, \bar{v})}, \quad \text { with } \bar{v}=v(h(\bar{\rho} ; \alpha, \beta)) .
$$

For $v \in \overline{\mathcal{F}}$ satisfying $h(\bar{\rho} ; \alpha, \beta)=\frac{\bar{N}(\bar{\rho}, v)}{\bar{D}(\bar{\rho}, v)}, v=\bar{v}$ for $\bar{\rho}$-a.e. $(k, l) \in\{A, B\} \times[0, \infty)$ or $k=\mathcal{I}$.

Proof: The proof is similar to that of Lemma 7.1. The counterpart of Lemma 7.2 is obtained by showing that for $(\alpha, \beta) \in$ CONE and $\varepsilon>0$ there exists a $t_{\varepsilon}>0$ such that, for all $\bar{\rho} \in \mathcal{M}_{1}\left(\mathbb{R}_{+} \cup \mathbb{R}_{+} \cup\{\mathcal{I}\}\right)$ and all $v \in \overline{\mathcal{F}}$ satisfying $\bar{D}(\bar{\rho}, v) \in\left(t_{\varepsilon}, \infty\right)$,

$$
\frac{\bar{N}(\bar{\rho}, v)}{\bar{D}(\bar{\rho}, v)} \leq \varepsilon .
$$

The proof of (7.21) is similar to the proof of Lemma 7.2 and relies mainly on Lemmas B.1(ii-iii) and on the limit given in Lemma C.1(ii).

It remains to show that $h(\bar{\rho} ; \alpha, \beta)=\frac{\bar{N}(\bar{\rho}, \bar{v})}{\bar{D}(\bar{\rho}, \bar{v})}$ and that $v \in \overline{\mathcal{F}}$ satisfying $h(\bar{\rho} ; \alpha, \beta)=$ $\frac{\bar{N}(\bar{\rho}, v)}{\bar{D}(\bar{\rho}, v)}$ necessarily satisfies $v=\bar{v}$ for $\bar{\rho}$-a.e. $(k, l) \in\{A, B\} \times[0, \infty)$ or $k=\mathcal{I}$. The proofs are similar to their counterparts in Lemma 7.1 and require the strict concavity of $u \mapsto u \widetilde{\kappa}(u, l)$ for $l \in \mathbb{R}$ and of $u \mapsto u \phi_{\mathcal{I}}(u)$, as well as the definition of $\bar{v}$ in $(3.8-3.10)$.

7.3. Step 3: lower bound. The inequality in (7.7) is a straightforward consequence of the following lemma.

Lemma 7.4. For all $(\alpha, \beta) \in \mathrm{CONE}, \bar{\rho} \in \overline{\mathcal{R}}_{p, M}$ and $v=\left(v_{A}, v_{B}, v_{\mathcal{I}}\right) \in \overline{\mathcal{F}}$ there exists $\rho \in \mathcal{R}_{p, M}$ and $u=\left(u_{\Theta}\right)_{\Theta \in \overline{\mathcal{V}}_{M}} \in \mathcal{F}_{\overline{\mathcal{V}}_{M}}$ satisfying

$$
\frac{N(\bar{\rho}, v)}{D(\bar{\rho}, v)} \leq \frac{N(\rho, u)}{D(\rho, u)} .
$$

Proof: Since $\bar{\rho} \in \overline{\mathcal{R}}_{p, M}$, there exist $\rho \in \mathcal{R}_{p, M}$ and $h \in \mathcal{E}$ such that $\bar{\rho}=G_{\rho, h}$. For $\Theta \in \overline{\mathcal{V}}_{M}$ and $k \in\{A, B\}$, set $d_{k, \Theta}=l_{k, \Theta} / h_{k, \Theta}$ if $h_{k, \Theta}>0$ and $d_{k, \Theta}=0$ otherwise. Put

$$
u_{\Theta}=h_{A, \Theta} v_{A, d_{A, \Theta}}+h_{B, \Theta} v_{B, d_{A, \Theta}}+h_{\mathcal{I}, \Theta} v_{\mathcal{I}}, \quad \Theta \in \overline{\mathcal{V}}_{M}
$$

To prove (7.23), we recall (4.61) and integrate (7.24) against $\rho$. Since $\bar{\rho}=G_{\rho, h}$, it follows that

$$
D(\bar{\rho}, v)=\int_{\overline{\mathcal{V}}_{M}} u_{\Theta} \rho(d \Theta)=D(\rho, u) .
$$

Since $h \in \mathcal{E}$ we can assert that

$$
\left(h_{A, \Theta}, h_{B, \Theta}, h_{\mathcal{I}, \Theta}\right),\left(h_{A, \Theta} v_{A, d_{A}, \Theta}, h_{B, \Theta} v_{B, d_{B}, \Theta}, h_{\mathcal{I}, \Theta} v_{\mathcal{I}}\right) \in \mathcal{L}\left(\Theta ; u_{\Theta}\right), \quad \Theta \in \overline{\mathcal{V}}_{M},
$$


which, with the help of (4.48), allows us to write

$$
\begin{aligned}
& u_{\Theta} \Psi\left(\Theta, u_{\Theta}\right) \geq h_{A, \Theta} v_{A, d_{A, \Theta}} \tilde{\kappa}\left(v_{A, d_{A, \Theta}}, d_{A, \Theta}\right) \\
& +h_{B, \Theta} v_{B, d_{B, \Theta}}\left[\tilde{\kappa}\left(v_{B, d_{B, \Theta}}, d_{B, \Theta}\right)+\frac{\beta-\alpha}{2}\right]+h_{\mathcal{I}, \Theta} v_{\mathcal{I}} \phi_{\mathcal{I}}\left(v_{\mathcal{I}} ; \alpha, \beta\right) .
\end{aligned}
$$

After integrating (7.27) against $\rho$ and using that $\bar{\rho}=G_{\rho, u}$, we obtain

$$
\begin{aligned}
\int_{\overline{\mathcal{V}}_{M}} u_{\Theta} \psi\left(\Theta, u_{\Theta}\right) \rho(d \Theta) & \geq\left[\int_{0}^{\infty} v_{A, l} \kappa\left(v_{A, l}, l\right) \rho_{A}(d l)\right. \\
& \left.+\int_{0}^{\infty} v_{B, l}\left[\kappa\left(v_{B, l}, l\right)+\frac{\beta-\alpha}{2}\right] \rho_{B}(d l)+\rho_{\mathcal{I}} v_{\mathcal{I}} \phi_{\mathcal{I}}\left(v_{\mathcal{I}} ; \alpha, \beta\right)\right] .
\end{aligned}
$$

Thus, (7.23) is immediate from (7.25) and (7.28).

7.4. Step 4: upper bound. The proof of (7.8) is a straightforward consequence of the following lemma.

Lemma 7.5. For all $(\alpha, \beta) \in \mathrm{CONE}, \rho \in \mathcal{R}_{p, M}$ and $u \in \mathcal{B}_{\overline{\mathcal{V}}_{M}}$, there exist $\bar{\rho} \in \overline{\mathcal{R}}_{p, M}$ and $v \in \overline{\mathcal{F}}$ such that

$$
\frac{N(\rho, u)}{D(\rho, u)} \leq \frac{N(\bar{\rho}, v)}{D(\bar{\rho}, v)} .
$$

Proof: Since $u \in \mathcal{B}_{\overline{\mathcal{V}}_{M}}$, Proposition G.1 in Appendix $\mathrm{G}$ allows us to state that there exist $h \in \mathcal{E}$ and $r \in \mathcal{U}(h)$ such that

$$
\begin{aligned}
u_{\Theta} \psi\left(\Theta, u_{\Theta}\right)=h_{A, \Theta} r_{A, \Theta} \tilde{\kappa}\left(r_{A, \Theta}, \frac{l_{A, \Theta}}{h_{A, \Theta}}\right) & +h_{B, \Theta} r_{B, \Theta}\left[\tilde{\kappa}\left(r_{B, \Theta}, \frac{l_{B, \Theta}}{h_{B, \Theta}}\right)+\frac{\beta-\alpha}{2}\right] \\
& +h_{\mathcal{I}, \Theta} r_{\mathcal{I}, \Theta} \phi_{\mathcal{I}}\left(r_{\mathcal{I}, \Theta}\right), \quad \forall \Theta \in \overline{\mathcal{V}}_{M},
\end{aligned}
$$

and

$$
h_{A, \Theta} r_{A, \Theta}+h_{B, \Theta} r_{B, \Theta}+h_{\mathcal{I}, \Theta} r_{\mathcal{I}, \Theta}=u_{\Theta}, \quad \forall \Theta \in \overline{\mathcal{V}}_{M}
$$

Define $\rho_{A, h}, \rho_{B, h}, \rho_{\mathcal{I}, h}$ to be the probability measures on $\overline{\mathcal{V}}_{M}$ given by

$$
\frac{d \rho_{k, h}}{d \rho}(\Theta)=\frac{h_{k, \Theta}}{\int_{\overline{\mathcal{V}}_{M}} h_{k, \Theta} \rho(d \Theta)}, \quad k \in\{A, B, \mathcal{I}\} .
$$

For $l \in \mathbb{R}_{+}$, set

$$
v_{A, l}=E_{\rho_{A, h}}\left[r_{A, \Theta} \mid \frac{l_{A, \Theta}}{h_{A, \Theta}}=l\right], \quad v_{B, l}=E_{\rho_{B, h}}\left[r_{B, \Theta} \mid \frac{l_{B, \Theta}}{h_{B, \Theta}}=l\right],
$$

and

$$
v_{\mathcal{I}}=E_{\rho_{\mathcal{I}, h}}\left[r_{\mathcal{I}, \Theta}\right] .
$$

The fact that $r \in \mathcal{U}(h)$ implies that $v_{\mathcal{I}} \geq 1$ and $v_{k, l} \geq 1+l$ for $l \in \mathbb{R}_{+}$and $k \in\{A, B\}$. Moreover, the Borel measurability of $\Theta \mapsto h_{k, \Theta}$ for $k \in\{A, B\}$ implies the Lebesgue measurability of $l \mapsto v_{k, l}$ for $k \in\{A, B\}$. Therefore, $\left(v_{A}, v_{B}, v_{\mathcal{I}}\right) \in \overline{\mathcal{F}}$.

By the concavity of $a \mapsto a \tilde{\kappa}(a, b)$ and $\mu \mapsto \mu \phi_{\mathcal{I}}(\mu)$, we obtain that

$$
\begin{aligned}
& E_{\rho_{A, h}}\left[r_{A, \Theta} \tilde{\kappa}\left(r_{A, \Theta}, l\right) \mid \frac{l_{A, \Theta}}{h_{A, \Theta}}=l\right] \leq v_{A, l} \widetilde{\kappa}\left(v_{A, l}, l\right), \\
& E_{\rho_{B, h}}\left[r_{B, \Theta}\left(\tilde{\kappa}\left(r_{B, \Theta}, l\right)+\frac{\beta-\alpha}{2}\right) \mid \frac{l_{B, \Theta}}{h_{B, \Theta}}=l\right] \leq v_{B, l}\left[\widetilde{\kappa}\left(v_{B, l}, l\right)+\frac{\beta-\alpha}{2}\right], \\
& E_{\rho_{\mathcal{I}, h}}\left[r_{\mathcal{I}, \Theta} \phi_{\mathcal{I}}\left(r_{\mathcal{I}, \Theta}\right)\right] \leq v_{\mathcal{I}} \phi_{\mathcal{I}}\left(v_{\mathcal{I}}\right) .
\end{aligned}
$$


Integrate (7.30) against $\rho$, to obtain

$$
\begin{aligned}
\int_{\overline{\mathcal{V}}_{M}} u_{\Theta} \psi\left(\Theta, u_{\Theta}\right) \rho(d \Theta)= & \int_{\overline{\mathcal{V}}_{M}} h_{A, \Theta} \rho(d \Theta) E_{\rho_{A, h}}\left[r_{A, \Theta} \widetilde{\kappa}\left(r_{A, \Theta}, \frac{l_{A, \Theta}}{h_{A, \Theta}}\right)\right] \\
& +\int_{\overline{\mathcal{V}}_{M}} h_{\mathcal{I}, \Theta} \rho(d \Theta) E_{\rho_{\mathcal{I}, h}}\left[r_{\mathcal{I}, \Theta} \phi_{\mathcal{I}}\left(r_{\mathcal{I}, \Theta}\right)\right] \\
& +\int_{\overline{\mathcal{V}}_{M}} h_{B, \Theta} \rho(d \Theta) E_{\rho_{B, h}}\left[r_{B, \Theta}\left(\widetilde{\kappa}\left(r_{B, \Theta}, \frac{l_{B, \Theta}}{h_{B, \Theta}}\right)+\frac{\beta-\alpha}{2}\right)\right] .
\end{aligned}
$$

Set $\bar{\rho}=G_{\rho, h}$. In the right-hand side of (7.36) take the conditional expectation with respect to $\frac{l_{A, \Theta}}{h_{A, \Theta}}$ and $\frac{l_{B, \Theta}}{h_{B} \Theta}$ in the first term and the second term, respectively. Then use the inequalities in (7.35), to obtain

$$
\begin{aligned}
\int_{\overline{\mathcal{V}}_{M}} u_{\Theta} \psi\left(\Theta, u_{\Theta}\right) & \rho(d \Theta) \leq \int_{0}^{\infty} v_{A, l} \widetilde{\kappa}\left(v_{A, l}, l\right) \bar{\rho}_{A}(d l) \\
& +\int_{0}^{\infty} v_{B, l}\left[\tilde{\kappa}\left(v_{B, l}, l\right)+\frac{\beta-\alpha}{2}\right] \bar{\rho}_{B}(d l)+\bar{\rho}_{\mathcal{I}} v_{\mathcal{I}} \phi_{\mathcal{I}}\left(v_{\mathcal{I}}, \alpha, \beta\right) .
\end{aligned}
$$

Similarly, integrate (7.31) against $\rho$ and take the conditional expectation with respect to $\frac{l_{A, \Theta}}{h_{A, \Theta}}$ and $\frac{l_{B, \Theta}}{h_{B, \Theta}}$, to obtain

$$
\int_{\overline{\mathcal{V}}_{M}} u_{\Theta} \rho(d \Theta)=\int_{0}^{\infty} v_{A, l} \bar{\rho}_{A}(d l)+\int_{0}^{\infty} v_{B, l} \bar{\rho}_{B}(d l)+\bar{\rho}_{\mathcal{I}} v_{\mathcal{I}} .
$$

At this point, (7.36) and (7.38) allow us to conclude that $N(\rho, u) / D(\rho, u) \leq$ $N(\bar{\rho}, v) / D(\bar{\rho}, v)$. Since $v \in \overline{\mathcal{F}}$, this completes the proof.

\section{Phase diagrams: proof of Theorems 3.1, 3.7 and 3.11}

8.1. Proof of Theorem 3.1. We first state and prove a proposition that compares $f, f_{\mathcal{D}}$ and $f_{\mathcal{D}_{2}}$, and deals with the regularity and the monotonicity of $f_{\mathcal{D}}$. Recall the definition of $\alpha^{*}$ in (3.20).

Proposition 8.1. (i) $f(\alpha, \beta)=f_{\mathcal{D}}(\alpha, \beta)$ for $(\alpha, \beta) \in \mathrm{CONE}: \beta \leq 0$.

(ii) $x \mapsto f_{\mathcal{D}}(x, 0)$ is continuous, convex and non-increasing on $[0, \infty)$.

(iii) $f_{\mathcal{D}}(x, 0)>f_{\mathcal{D}_{2}}$ for $x \in\left[0, \alpha^{*}\right)$ and $f_{\mathcal{D}}(x, 0)=f_{\mathcal{D}_{2}}$ for $x \in\left[\alpha^{*}, \infty\right)$.

Proof: (i) Note that for $(\alpha, \beta) \in \operatorname{CONE}: \beta \leq 0$ and $v \geq 1$ we have $\phi^{\mathcal{I}}(v, \alpha, \beta)=$ $\tilde{\kappa}(v, 0)$, because the Hamiltonian in (4.6) is always non-positive. Thus, (2.14) and (3.1) imply (i).

(ii) Since $(\alpha, \beta) \mapsto f(\alpha, \beta)$ is convex on $\mathbb{R}^{2}$ (being the pointwise limit of a sequence of convex functions; see (2.9)) and is everywhere finite, it is also continuous. Therefore (i) implies that $x \in[0, \infty) \mapsto f_{\mathcal{D}}(x, 0)$ is continous and convex. The monotonicity of $x \mapsto f_{\mathcal{D}}(x, 0)$ can be read off directly from (3.1).

(iii) It is obvious from (3.1) and (3.13) that $f_{\mathcal{D}}(x, 0) \geq f_{\mathcal{D}_{2}}$ for every $x \in[0, \infty)$. Recall (3.20). Since $x \mapsto f_{\mathcal{D}}(x, 0)$ is continuous and non-increasing, it follows that $f_{\mathcal{D}}(x, 0)>f_{\mathcal{D}_{2}}$ for $x \in\left[0, \alpha^{*}\right)$ and $f_{\mathcal{D}}(x, 0)=f_{\mathcal{D}_{2}}$ for $x \in\left[\alpha^{*}, \infty\right)$.

We are now ready to give the proof of Theorem 3.1.

Proof: (a) Pick $\alpha \geq 0$ and note that every element of $J_{\alpha}$ can be written in the form $(\alpha+\beta, \beta)$ (with $\beta \geq-\alpha / 2)$, so that $f_{\mathcal{D}}$ is constant and equal to $f_{\mathcal{D}}(\alpha, 0)$ on $J_{\alpha}$. By the convexity of $(\alpha, \beta) \mapsto f(\alpha, \beta)$ and by Proposition 8.1(i), we know that 
$g_{\alpha}: \beta \mapsto f(\alpha+\beta, \beta)-f_{\mathcal{D}}(\alpha, 0)$ is convex and equal to 0 when $\beta \leq 0$. Therefore $g_{\alpha}$ is non-decreasing, and we can define

$$
\beta_{c}(\alpha)=\inf \left\{\beta \geq 0: f(\alpha+\beta, \beta)>f_{\mathcal{D}}(\alpha, 0)\right\}
$$

so that $(\alpha+\beta, \beta) \in \mathcal{D}$ if and only if $\beta \leq \beta_{c}(\alpha)$. It remains to check that $\beta_{c}(\alpha)<\infty$.

To that aim, pick any $\bar{\rho} \in \overline{\mathcal{R}}_{p}$ such that $\bar{\rho}_{\mathcal{I}}>0$ and any $v \in \overline{\mathcal{B}}$ such that $v_{\mathcal{I}}>1$ and $\bar{D}(\bar{\rho}, v)<\infty$, recall (2.15), and note that $\lim _{\beta \rightarrow \infty} \bar{N}(\alpha+\beta, \beta ; \bar{\rho}, v)=\infty$ because $\lim _{\beta \rightarrow \infty} \phi_{\mathcal{I}}\left(v_{\mathcal{I}} ; \alpha+\beta, \beta\right)=\infty$. The last observation is obtained by considering a trajectory in $\mathcal{W}_{v_{\mathcal{I}} L}$ that starts at $(0,0)$ ends at $(L, 0)$, and in between stays in the $A$-solvent except when the microscopic disorder $\omega$ has 3 consecutive $B$-monomers, in which case the trajectory makes an excursion of size 3: one step south, one step east and one step north, inside the $B$-solvent. Such a trajectory has energy $\beta c L$ for some $c>0$.

(b) This is a straightforward consequence of the fact that $f_{\mathcal{D}}(\alpha, \beta)=f_{\mathcal{D}}(\alpha-\beta, 0)$ for $(\alpha, \beta) \in$ CONE.

\subsection{Proof of Theorem 3.\%.}

Proof: (a) We want to show that $\alpha^{*} \in(0, \infty)$. To that aim, we first prove that $f_{\mathcal{D}}(0,0)>f_{\mathcal{D}_{2}}$, which by the continuity of $x \mapsto f_{\mathcal{D}}(x, 0)$ implies that $\alpha^{*}>0$. It is easy to see that $p \delta_{A, 0}(d l)+(1-p) \delta_{B, 0}(d l) \in \overline{\mathcal{R}}_{p}$, since this corresponds to trajectories travelling along the $x$-axis while staying on one side. Thus, (3.1) implies that $f_{\mathcal{D}}(0,0) \geq \tilde{\kappa}\left(u^{*}, 0\right)$, where $u^{*}$ is the unique maximizer of $u \mapsto \tilde{\kappa}(u, 0)$ on $[1, \infty)$. Moreover, by Lemma B.1(ii), we have $\tilde{\kappa}(u, l) \leq \tilde{\kappa}\left(u^{*}, 0\right)$ for every $l \in[0, \infty)$, $u \geq 1+l$ and $(u, l) \neq\left(u^{*}, 0\right)$. Since $\delta_{A, 0}(d l)$ does not belong to $\overline{\mathcal{R}}_{p}$, it follows that $f_{\mathcal{D}_{2}}<f_{\mathcal{D}}(0,0)$, and therefore the continuity of $x \mapsto f_{\mathcal{D}}(x, 0)$ implies that $\alpha^{*}>0$.

It remains to show that $\alpha^{*}<\infty$. Recall Hypothesis 3.4. We argue by contradiction. Assume that $f_{\mathcal{D}}(n, 0)>f_{\mathcal{D}_{2}}$ for all $n \in \mathbb{N}$. Then Hypothesis 3.2 and Lemma 7.3 tell us that there exists a sequence $\left(\bar{\rho}_{n}\right)_{n \in \mathbb{N}}$ in $\mathcal{T}_{p}$ such that

$$
f(n, 0)=f_{\mathcal{D}}(n, 0)=\frac{\bar{N}_{\mathcal{D}}\left(\bar{\rho}_{n}, v_{n} ; n, 0\right)}{\bar{D}_{\mathcal{D}}\left(\bar{\rho}_{n}, v_{n}\right)}>f_{\mathcal{D}_{2}}>0, \quad n \in \mathbb{N},
$$

with $v_{n}=v\left(f_{\mathcal{D}}(n, 0)\right)$, where we recall (3.8-3.10). For simplicity, we write $f_{2}=f_{\mathcal{D}_{2}}$ and $\bar{v}=v\left(f_{2}\right)$ (recall (3.8-3.10)) until the end of the proof. Since $f_{\mathcal{D}}(n, 0)>f_{2}$ for $n \in \mathbb{N}$, Lemma B.3(ii) yields $v_{n, A, l} \leq \bar{v}_{A, l}$ for $l \in[0, \infty), n \in \mathbb{N}$. Note that Lemma B. 3 is stated for fixed $(\alpha, \beta) \in$ CONE, which is not the case here because $(\alpha, \beta)=(n, 0)$. However, in the present setting Lemma B.3(ii) remains true for $v_{A}$ since, by definition, the value taken by $v_{A, l}(c)$ for $l \in[0, \infty)$ and $c \in(0, \infty)$ does not depend on $(\alpha, \beta)$.

We can write

$$
\begin{aligned}
f_{\mathcal{D}}(n, 0)-f_{2}= & \frac{\int_{0}^{\infty} v_{n, A, l}\left[\tilde{\kappa}\left(v_{n, A, l}, l\right)-f_{2}\right]\left(\bar{\rho}_{n, A}+\bar{\rho}_{n, \mathcal{I}} \delta_{0}\right)(d l)}{D_{\mathcal{D}}\left(\bar{\rho}_{n}, v_{n}\right)} \\
& +\frac{\int_{0}^{\infty} v_{n, B, l}\left[\tilde{\kappa}\left(v_{n, B, l}, l\right)-\frac{n}{2}-f_{2}\right] \bar{\rho}_{n, B}(d l)}{D_{\mathcal{D}}\left(\bar{\rho}_{n}, v_{n}\right)},
\end{aligned}
$$

and the concavity of $v \mapsto v \tilde{\kappa}(v, l)$, together with the fact that $v_{n, A, l} \leq \bar{v}_{A, l}$ for all $l \in[0, \infty)$ and $\partial_{v}(v \tilde{\kappa}(v, l))\left(\bar{v}_{A, l}\right)=f_{2}$, implies that

$$
\bar{v}_{A, l} \tilde{\kappa}\left(\bar{v}_{A, l}, l\right)-v_{n, A, l} \tilde{\kappa}\left(v_{n, A, l}, l\right) \geq f_{2}\left(\bar{v}_{A, l}-v_{n, A, l}\right) .
$$


Since $\tilde{\kappa}$ is uniformly bounded from above and $v_{n, B, l} \geq 1+l$ for every $l \in[0, \infty)$, we can claim that, for $n$ large enough,

$$
v_{n, B, l}\left[\tilde{\kappa}\left(v_{n, B, l}, l\right)-\frac{n}{2}-f_{2}\right] \leq-\frac{n}{4}(1+l), \quad l \in[0, \infty) .
$$

Consequently, (8.2) and (8.3-8.5) allow us to write

$$
\int_{0}^{\infty} \bar{v}_{A, l}\left[\tilde{\kappa}\left(\bar{v}_{A, l}, l\right)-f_{2}\right]\left(\bar{\rho}_{n, A}+\bar{\rho}_{n, \mathcal{I}} \delta_{0}\right)(d l)-\frac{n}{4} \int_{0}^{\infty} 1+l \bar{\rho}_{n, B}(d l)>0
$$

for $n$ large enough, which clearly contradicts Hypothesis 3.4 because $\bar{\rho}_{n} \in \mathcal{T}_{p}$ for $n \in \mathbb{N}$. The proof is therefore complete.

(b-c) By the definition of $\mathcal{D}, \mathcal{D}_{1}$ and $\mathcal{D}_{2}$ in (3.4), (3.15) and (3.16), we know that $\mathcal{D}=\mathcal{D}_{1} \cup \mathcal{D}_{2}$ and $\mathcal{D}_{1} \cap \mathcal{D}_{2}=\emptyset$. Thus, Theorem 3.1(a) implies that (b) and (c) will be proven once we show that $J_{\alpha} \cap \mathcal{D}_{2}=\emptyset$ for $\alpha \in\left[0, \alpha^{*}\right)$ and $J_{\alpha} \cap \mathcal{D}_{1}=\emptyset$ for $\alpha \in\left[\alpha^{*}, \infty\right)$. Moreover, Theorem 3.1(b) tells us that $f_{\mathcal{D}}$ is constant and equal to $f_{\mathcal{D}}(\alpha, 0)$ on each $J_{\alpha}$ with $\alpha \in[0, \infty)$. Consequently it suffices to show that $f_{\mathcal{D}}(\alpha, 0)>f_{\mathcal{D}_{2}}$ for $\alpha \in\left[0, \alpha^{*}\right)$ and $f_{\mathcal{D}}(\alpha, 0)=f_{\mathcal{D}_{2}}$ for $\alpha \in\left[\alpha^{*}, \infty\right)$. But this is precisely what Proposition 8.1(iii) states.

(d) Pick $\alpha \in[0, \infty)$ and assume that Hypothesis 3.2 holds. Then there exists a $\bar{\rho}_{\alpha} \in \mathcal{O}_{p, \alpha, 0}$ such that $\bar{\rho}_{\alpha, \mathcal{I}}>0$. Set $\bar{v}=v\left(f_{\mathcal{D}}(\alpha, 0)\right)$ and

$$
\tilde{\beta}_{c}(\gamma(\alpha))=\inf \left\{\beta>0: \phi_{\mathcal{I}}\left(\bar{v}_{A, 0} ; \beta+\alpha, \beta\right)>\tilde{\kappa}\left(\bar{v}_{A, 0}, 0\right)\right\} .
$$

The proof will be complete as soon as we show that $\tilde{\beta}_{c}(\gamma(\alpha))=\beta_{c}(\gamma(\alpha)$ ) (recall (3.6)). Note that, by the convexity of $\beta \rightarrow \phi_{\mathcal{I}}\left(\bar{v}_{A, 0} ; \alpha+\beta, \beta\right)$, and since $\phi_{\mathcal{I}}\left(\bar{v}_{A, 0} ; \beta+\right.$ $\alpha, \beta)=\tilde{\kappa}\left(\bar{v}_{A, 0}, 0\right)$ for $\beta \leq 0$, we necessarily have that $\phi_{\mathcal{I}}\left(\bar{v}_{A, 0} ; \alpha+\beta, \beta\right)>\tilde{\kappa}\left(\bar{v}_{A, 0}, 0\right)$ for all $\beta>\tilde{\beta}_{c}(\gamma(\alpha))$. From Propositions 8.1(i) and F.1(2), we have that

$$
f(\alpha, 0)=f_{\mathcal{D}}(\alpha, 0)=\frac{\bar{N}_{\mathcal{D}}\left(\bar{\rho}_{\alpha}, \bar{v}\right)}{\bar{D}_{\mathcal{D}}\left(\bar{\rho}_{\alpha}, \bar{v}\right)},
$$

and

$\bar{N}_{\mathcal{D}}\left(\bar{\rho}_{\alpha}, \bar{v}\right)=\int_{0}^{\infty} \bar{v}_{A, l} \tilde{\kappa}\left(\bar{v}_{A, l}, l\right)\left[\bar{\rho}_{\alpha, A}+\bar{\rho}_{\alpha, \mathcal{I}} \delta_{0}\right](d l)+\int_{0}^{\infty} \bar{v}_{B, l}\left[\tilde{\kappa}\left(\bar{v}_{B, l}, l\right)-\frac{\alpha}{2}\right] \bar{\rho}_{\alpha, B}(d l)$.

By the definition of $\bar{v}=v\left(f_{\mathcal{D}}(\alpha, 0)\right)$ in (3.8-3.10), we have that $\partial_{v}(v \tilde{\kappa}(v, 0))\left(\bar{v}_{A, 0}\right)=$ $f_{\mathcal{D}}(\alpha, 0)$. For notational reasons we suppress the dependence on $\alpha$ of $f_{\mathcal{D}}$.

First, assume that $\phi_{\mathcal{I}}\left(\bar{v}_{A, 0} ; \beta+\alpha, \beta\right)=\tilde{\kappa}\left(\bar{v}_{A, 0}, 0\right)$ (we also suppress the dependence on $(\beta+\alpha, \beta))$. Then, since $v \rightarrow v \phi_{\mathcal{I}}(v)$ and $v \rightarrow v \tilde{\kappa}(v, 0)$ are both concave and $\phi_{\mathcal{I}}(v) \geq \tilde{\kappa}(v, 0)$ for all $v \geq 1$, we have that $v \rightarrow v \phi_{\mathcal{I}}(v)$ is differentiable at $\bar{v}_{A, 0}$ and

$$
\partial_{v}[v \tilde{\kappa}(v, 0)]\left(\bar{v}_{A, 0}\right)=\partial_{v}\left[v \phi_{\mathcal{I}}(v)\right]\left(\bar{v}_{A, 0}\right)=f_{\mathcal{D}} .
$$

Thus, for any $\bar{\rho} \in \overline{\mathcal{R}}_{p}$ and $v \in \overline{\mathcal{B}}$, we set $\tilde{v} \in \overline{\mathcal{B}}$ such that $\tilde{v} \equiv v$, except for $\tilde{v}_{\mathcal{I}}$, which takes the value $\bar{v}_{A, 0}$. In other words,

$$
\begin{aligned}
\frac{\bar{N}(\bar{\rho}, v)}{\bar{D}(\bar{\rho}, v)} & =\frac{\bar{N}_{\mathcal{D}}(\bar{\rho}, \tilde{v})+\bar{\rho}_{\mathcal{I}}\left[v_{\mathcal{I}} \phi_{\mathcal{I}}\left(v_{\mathcal{I}}\right)-\bar{v}_{A, 0} \tilde{\kappa}\left(\bar{v}_{A, 0}, 0\right)\right]}{\bar{D}_{\mathcal{D}}(\bar{\rho}, \tilde{v})+\bar{\rho}_{\mathcal{I}}\left[v_{\mathcal{I}}-\bar{v}_{A, 0}\right]} \\
& \leq \frac{\bar{N}_{\mathcal{D}}(\bar{\rho}, \tilde{v})+\bar{\rho}_{\mathcal{I}} f_{\mathcal{D}}\left(v_{\mathcal{I}}-\bar{v}_{A, 0}\right)}{\bar{D}_{\mathcal{D}}(\bar{\rho}, \tilde{v})+\bar{\rho}_{\mathcal{I}}\left(v_{\mathcal{I}}-\bar{v}_{A, 0}\right)}
\end{aligned}
$$


where we use (8.10), the concavity of $v \rightarrow v \phi_{\mathcal{I}}(v)$ and the fact that $\phi_{\mathcal{I}}\left(\bar{v}_{A, 0}\right)=$ $\tilde{\kappa}\left(\bar{v}_{A, 0}, 0\right)$ by assumption. At this stage we recall that, by definition, $\frac{\bar{N}_{\mathcal{D}}(\bar{\rho}, \tilde{v})}{\bar{D}_{\mathcal{D}}(\bar{\rho}, \tilde{v})} \leq f_{\mathcal{D}}$. Hence (8.11) entails that $\frac{N(\bar{\rho}, v)}{D(\bar{\rho}, v)} \leq f_{\mathcal{D}}$. Thus, $\beta_{c}(\gamma(\alpha)) \geq \tilde{\beta}_{c}(\gamma(\alpha))$.

The other inequality is much easier. Indeed, if we consider $\beta$ such that $\phi_{\mathcal{I}}\left(\bar{v}_{A, 0} ; \alpha+\beta, \beta\right)>\tilde{\kappa}\left(\bar{v}_{A, 0}, 0\right)$, then $\bar{N}\left(\bar{\rho}_{\alpha}, \bar{v}\right)>\bar{N}_{\mathcal{D}}\left(\bar{\rho}_{\alpha}, \bar{v}\right)$ because $\bar{\rho}_{\mathcal{I}, \alpha}>0$. As a consequence, $f(\alpha+\beta, \beta)>f_{\mathcal{D}}(\alpha, 0)$, so that $\beta>\beta_{c}(\gamma(\alpha))$, and therefore $\beta_{c}(\gamma(\alpha)) \leq \tilde{\beta}_{c}(\gamma(\alpha))$.

(e) We recall that for $\alpha \in\left[\alpha^{*}, \infty\right)$ we have $\bar{v}=v\left(f_{D_{2}}\right)$ and therefore $\bar{v}_{A, 0}$ is constant. In (c) we proved that $\beta_{c}(\gamma(\alpha))=\tilde{\beta}_{c}(\gamma(\alpha))$ on $\left[\alpha^{*}, \infty\right)$. The definition of $\tilde{\beta}_{c}(\gamma(\alpha))$ in (8.7) can be extended to $\alpha \in[0, \infty)$. Since $\alpha^{*}>0$, the proof of (d) will be complete once we show that $\alpha \mapsto \tilde{\beta}_{c}(\gamma(\alpha))$ is concave, continuous and non-decreasing on $(0, \infty)$ and that $\lim _{\alpha \rightarrow \infty} \tilde{\beta}_{c}(\gamma(\alpha))<\infty$.

By using the same argument as the one we used in the proof of Theorem 3.1(a), we can claim that $\lim _{\beta \rightarrow \infty} \phi_{\mathcal{I}}\left(\bar{v}_{A, 0} ; \alpha+\beta, \beta\right)=\infty$ for every $\alpha \in[0, \infty)$. Consequently, $\tilde{\beta}_{c}(\gamma(\alpha)) \in[0, \infty)$ for every $\alpha \in[0, \infty)$. Moreover, the convexity of $(\alpha, \beta) \mapsto$ $\phi_{\mathcal{I}}\left(\bar{v}_{A, 0} ; \alpha, \beta\right)$ implies the convexity of $(\alpha, \beta) \mapsto \phi_{\mathcal{I}}\left(\bar{v}_{A, 0} ; \alpha+\beta, \beta\right)-\tilde{\kappa}\left(\bar{v}_{A, 0}, 0\right)$, which is also non-negative. Therefore, the set $\left\{(\alpha, \beta): \alpha \in[0, \infty), \beta \in\left[-\frac{\alpha}{2}, \tilde{\beta}_{c}(\gamma(\alpha))\right]\right\}$ is convex, and consequently $\alpha \mapsto \tilde{\beta}_{c}(\gamma(\alpha))$ is concave on $[0, \infty)$. This concavity yields that $\alpha \mapsto \tilde{\beta}_{c}(\gamma(\alpha))$ is continuous on $(0, \infty)$, and since it is bounded from below by 0 , also that it is non-decreasing.

It remains to show that $\lim _{\alpha \rightarrow \infty} \tilde{\beta}_{c}(\gamma(\alpha))<\infty$. To that aim, we define $\tilde{\beta}_{c}(\infty)$ by choosing $\alpha=\infty$ in (8.7). Since $\phi_{\mathcal{I}}\left(\bar{v}_{A, 0} ; \infty, \beta\right) \leq \phi_{\mathcal{I}}\left(\bar{v}_{A, 0} ; \alpha+\beta, \beta\right)$ for every $\alpha \geq 0$ and $\beta \in\left[-\frac{\alpha}{2}, \infty\right)$, it follows that $\tilde{\beta}_{c}(\gamma(\alpha)) \leq \tilde{\beta}_{c}(\infty)$ for every $\alpha \in(0, \infty)$. Therefore it suffices to prove that $\tilde{\beta}_{c}(\infty)<\infty$. But this is a consequence of the fact that $\lim _{\alpha \rightarrow \infty} \phi_{\mathcal{I}}\left(\bar{v}_{A, 0} ; \infty, \beta\right)=\infty$. This limit is obtained by using again the same argument as the one we used in the proof of Theorem 3.1(a).

(f) This is a straightforward consequence of the fact that $f=f_{\mathcal{D}}$ on $\mathcal{D}_{1}$ and $f_{\mathcal{D}}$ is a function of $\alpha-\beta$.

(g) This is a direct consequence of the definition of the $\mathcal{D}_{2}$-phase in (3.16) and the fact that $f_{\mathcal{D}_{2}}$ does not depend on $\alpha$ and $\beta$ (see (3.13)).

8.3. Proof of Theorem 3.11. The proof of Theorem 3.11 has much in common with that of Theorem 3.7 in Section 8.2. For this reason we only focus on the points that need to be adapted from the proof of Theorem 3.7.

Proof: (a) The proof of $\bar{\alpha}^{*} \in(0, \infty)$ follows the same scheme as the proof of Theorem 3.7(a). The bound $f_{\mathcal{D}}(0,0) \geq \tilde{\kappa}\left(u^{*}, 0\right)$ remains valid ( $u^{*}$ being the unique maximizer of $u \mapsto \tilde{\kappa}(u, 0))$. Moreover, $\left\{\bar{\rho} \in \overline{\mathcal{R}}_{p}: K_{B}(\bar{\rho})=K_{p}\right\}$ does not contain any element of the form $x \delta_{A, 0}(d l)+(1-x) \delta_{B, 0}(d l)$, since the fraction of horizontal steps taken in solvent $B$ can obviously be reduced by allowing the path to sometimes travel in solvent $A$ with a non-zero slope. This implies that $f_{\mathcal{D}}(0,0)>f_{\mathcal{D}_{2}}(0,0)$, and therefore $\bar{\alpha}^{*}>0$.

The upper bound is also similar to that of Theorem 3.7(a). The only difference is that $f_{\mathcal{D}_{2}}$ depends on $n$, so that we write $f_{2}(n)$ as well as $\bar{v}_{n}=v\left(f_{2}(n)\right)$. Both (8.3) and (8.4) are still true, whereas some attention is needed to adapt (8.5) since $f_{2}$ depends on $n$. However, it suffices to pick any $\bar{\rho} \in \overline{\mathcal{R}}_{p} \backslash \mathcal{T}_{p}$ such that $K_{A}(\bar{\rho})+$ 
$K_{B}(\bar{\rho})<\infty$ and $\bar{v}^{*} \in \mathcal{B}$, and such that $\bar{v}_{k, l}^{*}=1+l$ for $(k, l) \in\{A, B\} \times[0, \infty)$ and $\bar{v}_{\mathcal{I}}^{*}=1$, to obtain that

$$
f_{2}(n)=f_{\mathcal{D}}(n, 0) \geq \frac{\bar{N}_{\mathcal{D}}\left(\bar{\rho}, \bar{v}^{*}\right)}{\bar{D}_{\mathcal{D}}\left(\bar{\rho}, \bar{v}^{*}\right)} \geq c_{1}-\frac{c_{2}}{2} n,
$$

where $c_{1} \in \mathbb{R}$ and $c_{2}=\int_{0}^{\infty}(1+l) \bar{\rho}_{B}(d l) / \bar{D}_{\mathcal{D}}\left(\bar{\rho}, \bar{v}^{*}\right)$. Since $p<p_{c}$ and $\bar{\rho} \in \overline{\mathcal{R}}_{p} \backslash \mathcal{T}_{p}$, it follows that $K_{A}(\bar{\rho})>0$ and $K_{B}(\bar{\rho})>0$, and hence $c_{2} \in(0,1)$. Thus, (8.5) still holds with a right-hand side of the form $-\left(\frac{1}{4}-\frac{c_{2}}{4}\right)(1+l)$, which contradicts Hypothesis 3.9 and completes the proof.

(b) The proof is literally the same as that of Theorem 3.7(b-c-d).

(c) This is again a consequence of the fact that $f=f_{\mathcal{D}}$ on $\mathcal{D}$ and that $f_{\mathcal{D}}$ is a function of $\alpha-\beta$.

\section{Appendix A. Uniform convergence of path entropies}

In Appendix A.1 we state a basic lemma (Lemma A.1) about uniform convergence of path entropies in a single column. This lemma is proved with the help of three additional lemmas (Lemmas A.2-A.4), which are proved in Appendix A.3. The latter ends with an elementary lemma (Lemma B.1) that allows us to extend path entropies from rational to irrational parameter values. In Appendix A.2, we extend Lemma A.1 to entropies associated with sets of paths fullfilling certain restrictions on their vertical displacement.

A.1. Basic lemma. We recall the definition of $\widetilde{\kappa}_{L}, L \in \mathbb{N}$, in (4.2) and $\widetilde{\kappa}$ in (4.3).

Lemma A.1. For every $\varepsilon>0$ there exists an $L_{\varepsilon} \in \mathbb{N}$ such that

$$
\left|\tilde{\kappa}_{L}(u, l)-\tilde{\kappa}(u, l)\right| \leq \varepsilon \text { for } L \geq L_{\varepsilon} \text { and }(u, l) \in \mathcal{H}_{L} .
$$

Proof: With the help of Lemma A.2 below we get rid of those $(u, l) \in \mathcal{H} \cap \mathbb{Q}^{2}$ with $u$ large, i.e., we prove that $\lim _{u \rightarrow \infty} \kappa_{L}(u, l)=0$ uniformly in $L \in \mathbb{N}$ and $(u, l) \in \mathcal{H}_{L}$. Lemma A.3 in turn deals with the moderate values of $u$, i.e., $u$ bounded away from infinity and $1+|l|$. Finally, with Lemma A.4 we take into account the small values of $u$, i.e., $u$ close to $1+|l|$. To ease the notation we set, for $\eta \geq 0$ and $U>1$,

$$
\begin{aligned}
& \mathcal{H}_{L, \eta, U}=\left\{(u, l) \in \mathcal{H}_{L}: 1+|l|+\eta \leq u \leq U\right\}, \\
& \mathcal{H}_{\eta, U}=\{(u, l) \in \mathcal{H}: 1+|l|+\eta \leq u \leq U\} .
\end{aligned}
$$

Lemma A.2. For every $\varepsilon>0$ there exists an $U_{\varepsilon}>1$ such that

$$
\frac{1}{u L} \log \left|\left\{\pi \in \mathcal{W}_{u L}: \pi_{u L, 1}=L\right\}\right| \leq \varepsilon \quad \forall L \in \mathbb{N}, u \in 1+\frac{\mathbb{N}}{L}: u \geq U_{\varepsilon} .
$$

Lemma A.3. For every $\varepsilon>0, \eta>0$ and $U>1$ there exists an $L_{\varepsilon, \eta, U} \in \mathbb{N}$ such that

$$
\left|\tilde{\kappa}_{L}(u, l)-\tilde{\kappa}(u, l)\right| \leq \varepsilon \quad \forall L \geq L_{\varepsilon, \eta, U},(u, l) \in \mathcal{H}_{L, \eta, U} .
$$

Lemma A.4. For every $\varepsilon>0$ there exist $\eta_{\varepsilon} \in\left(0, \frac{1}{2}\right)$ and $L_{\varepsilon} \in \mathbb{N}$ such that

$$
\left|\tilde{\kappa}_{L}(u, l)-\tilde{\kappa}_{L}(u+\eta, l)\right| \leq \varepsilon \quad \forall L \geq L_{\varepsilon},(u, l) \in \mathcal{H}_{L}, \eta \in\left(0, \eta_{\varepsilon}\right) \cap \frac{2 \mathbb{N}}{L} .
$$


Note that, after letting $L \rightarrow \infty$ in Lemma A.4, we get

$$
|\tilde{\kappa}(u, l)-\tilde{\kappa}(u+\eta, l)| \leq \varepsilon \quad \forall(u, l) \in \mathcal{H} \cap \mathbb{Q}^{2}, \eta \in\left(0, \eta_{\varepsilon}\right) \cap \mathbb{Q} .
$$

Pick $\varepsilon>0$ and $\eta_{\varepsilon} \in\left(0, \frac{1}{2}\right)$ as in Lemma A.4. Note that Lemmas A.2-A.3 yield that, for $L$ large enough, (A.1) holds on $\left\{(u, l) \in \mathcal{H}_{L}: u \geq 1+|l|+\frac{\eta_{\varepsilon}}{2}\right\}$. Next, pick $L \in \mathbb{N},(u, l) \in \mathcal{H}_{L}: u \leq 1+|l|+\frac{\eta_{\varepsilon}}{2}$ and $\eta_{L} \in\left(\frac{\eta_{\varepsilon}}{2}, \eta_{\varepsilon}\right) \cap \frac{2 \mathbb{N}}{L}$, and write

$$
\left|\tilde{\kappa}_{L}(u, l)-\tilde{\kappa}(u, l)\right| \leq A+B+C,
$$

where

$$
\begin{aligned}
A & =\left|\tilde{\kappa}_{L}(u, l)-\tilde{\kappa}_{L}\left(u+\eta_{L}, l\right)\right|, \\
B & =\left|\tilde{\kappa}_{L}\left(u+\eta_{L}, l\right)-\tilde{\kappa}\left(u+\eta_{L}, l\right)\right|, \\
C & =\left|\tilde{\kappa}\left(u+\eta_{L}, l\right)-\tilde{\kappa}(u, l)\right| .
\end{aligned}
$$

By (A.6), it follows that $C \leq \varepsilon$. As mentioned above, the fact that $\left(u+\eta_{L}, l\right) \in \mathcal{H}_{L}$ and $u+\eta_{L} \geq|l|+\frac{\eta_{\varepsilon}}{2}$ implies that, for $L$ large enough, $B \leq \varepsilon$ uniformly in $(u, l) \in$ $\mathcal{H}_{L}: u \leq 1+|l|+\frac{\eta_{\varepsilon}}{2}$. Finally, from Lemma A.4 we obtain that $A \leq \varepsilon$ for $L$ large enough, uniformly in $(u, l) \in \mathcal{H}_{L}: u \leq 1+|l|+\frac{\eta_{\varepsilon}}{2}$. This completes the proof of Lemma A.1.

A.2. A generalization of Lemma A.1. In Section 6 we sometimes needed to deal with subsets of trajectories of the following form. Recall (4.1), pick $L \in \mathbb{N},(u, l) \in$ $\mathcal{H}_{L}$ and $B_{0}, B_{1} \in \frac{Z}{L}$ such that

$$
B_{1} \geq 0 \vee l \geq 0 \wedge l \geq B_{0} \text { and } \quad B_{1}-B_{0} \geq 1 \text {. }
$$

Denote by $\widetilde{\mathcal{W}}_{L}\left(u, l, B_{0}, B_{1}\right)$ the subset of $\mathcal{W}_{L}(u, l)$ containing those trajectories that are constrained to remain above $B_{0} L$ and below $B_{1} L$ (see Fig. A.16), i.e.,

$$
\widetilde{\mathcal{W}}_{L}\left(u, l, B_{0}, B_{1}\right)=\left\{\pi \in \mathcal{W}_{L}(u, l): B_{0} L<\pi_{i, 2}<B_{1} L \text { for } i \in\{1, \ldots, u L-1\}\right\},
$$

and let

$$
\widetilde{\kappa}_{L}\left(u, l, B_{0}, B_{1}\right)=\frac{1}{u L} \log \left|\widetilde{\mathcal{W}}_{L}\left(u, l, B_{0}, B_{1}\right)\right|
$$

be the entropy per step carried by the trajectories in $\widetilde{\mathcal{W}}_{L}\left(u, l, B_{0}, B_{1}\right)$. With Lemma A.5 below we prove that the effect on the entropy of the restriction induced by $B_{0}$ and $B_{1}$ in the set $\widetilde{\mathcal{W}}_{L}(u, l)$ vanishes uniformly as $L \rightarrow \infty$.

Lemma A.5. For every $\varepsilon>0$ there exists an $L_{\varepsilon} \in \mathbb{N}$ such that, for $L \geq L_{\varepsilon}$, $(u, l) \in \mathcal{H}_{L}$ and $B_{0}, B_{1} \in \mathbb{Z} / L$ satisfying $B_{1}-B_{0} \geq 1, B_{1} \geq \max \{0, l\}$ and $B_{0} \leq \min \{0, l\}$

$$
\left|\tilde{\kappa}_{L}\left(u, l, B_{0}, B_{1}\right)-\tilde{\kappa}_{L}(u, l)\right| \leq \varepsilon .
$$

Proof: The key fact is that $B_{1}-B_{0} \geq 1$. The vertical restrictions $B_{1} \geq \max \{0, l\}$ and $B_{0} \leq \min \{0, l\}$ gives polynomial corrections in the computation of the entropy, but these corrections are harmless because $\left(B_{1}-B_{0}\right) L$ is large.

\section{A.3. Proofs of Lemmas A.2-A.4.}




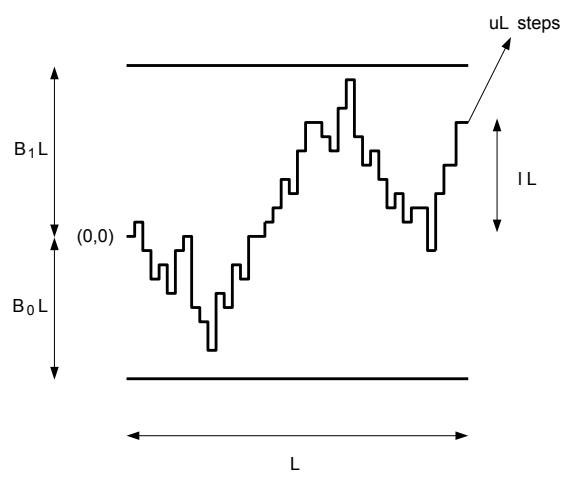

FiguRE A.16. A trajectory in $\widetilde{\mathcal{W}}_{L}\left(u, l, B_{0}, B_{1}\right)$.

A.3.1. Proof of Lemma A.2. The proof relies on the following expression:

$$
v_{u, L}=\left|\left\{\pi \in \mathcal{W}_{u L}: \pi_{u L, 1}=L\right\}\right|=\sum_{r=1}^{L+1}\left(\begin{array}{c}
L+1 \\
r
\end{array}\right)\left(\begin{array}{c}
(u-1) L \\
r
\end{array}\right) 2^{r}
$$

where $r$ stands for the number of vertical stretches made by the trajectory (a vertical stretch being a maximal sequence of consecutive vertical steps). Stirling's formula allows us to assert that there exists a $g:[1, \infty) \rightarrow(0, \infty)$ satisfying $\lim _{u \rightarrow \infty} g(u)=0$ such that

$$
\left(\begin{array}{c}
u L \\
L
\end{array}\right) \leq e^{g(u) u L}, \quad u \geq 1, L \in \mathbb{N} .
$$

Equations (A.13-A.14) complete the proof.

A.3.2. Proof of Lemma A.3. We first note that, since $u$ is bounded from above, it is equivalent to prove (A.4) with $\tilde{\kappa}_{L}$ and $\tilde{\kappa}$, or with $G_{L}$ and $G$ given by

$$
G(u, l)=u \tilde{\kappa}(u, l), \quad G_{L}(u, l)=u \tilde{\kappa}_{L}(u, l), \quad(u, l) \in \mathcal{H}_{L} .
$$

Via concatenation of trajectories, it is straightforward to prove that $G$ is $\mathbb{Q}$-concave on $\mathcal{H} \cap \mathbb{Q}^{2}$, i.e.,

$$
\begin{aligned}
G\left(\lambda\left(u_{1}, l_{1}\right)+(1-\lambda)\left(u_{2}, l_{2}\right)\right) \geq \lambda G\left(u_{1}, l_{1}\right)+(1-\lambda) G\left(u_{2}, l_{2}\right), & \\
& \lambda \in \mathbb{Q}_{[0,1]},\left(u_{1}, l_{1}\right),\left(u_{2}, l_{2}\right) \in \mathcal{H} \cap \mathbb{Q}^{2} .
\end{aligned}
$$

Therefore $G$ is Lipschitz on every $K \cap \mathcal{H} \cap \mathbb{Q}^{2}$ with $K \subset \mathcal{H}^{0}$ (the interior of $\mathcal{H}$ ) compact. Thus, $G$ can be extended on $\mathcal{H}^{0}$ to a function that is Lipschitz on every compact subset in $\mathcal{H}^{0}$.

Pick $\eta>0, M>1, \varepsilon>0$, and choose $L_{\varepsilon} \in \mathbb{N}$ such that $1 / L_{\varepsilon} \leq \varepsilon$. Since $\mathcal{H}_{\eta, M} \subset \mathcal{H}^{0}$ is compact, there exists a $c>0$ (depending on $\eta, M$ ) such that $G$ is $c$-Lipschitz on $\mathcal{H}_{\eta, M}$. Moreover, any point in $\mathcal{H}_{\eta, M}$ is at distance at most $\varepsilon$ from the finite lattice $\mathcal{H}_{L_{\varepsilon}, \eta, M}$. Lemma 4.1 therefore implies that there exists a $q_{\varepsilon} \in \mathbb{N}$ satisfying

$$
\left|G_{q L_{\varepsilon}}(u, l)-G(u, l)\right| \leq \varepsilon \quad \forall(u, l) \in \mathcal{H}_{L_{\varepsilon}, \eta, M}, q \geq q_{\varepsilon} .
$$


Let $L^{\prime}=q_{\varepsilon} L_{\varepsilon}$, and pick $q \in \mathbb{N}$ to be specified later. Then, for $L \geq q L^{\prime}$ and $(u, l) \in \mathcal{H}_{L, \eta, M}$, there exists an $\left(u^{\prime}, l^{\prime}\right) \in \mathcal{H}_{L_{\varepsilon}, \eta, M}$ such that $\left|(u, l)-\left(u^{\prime}, l^{\prime}\right)\right|_{\infty} \leq \varepsilon$, $u>u^{\prime},|l| \geq\left|l^{\prime}\right|$ and $u-u^{\prime} \geq|l|-\left|l^{\prime}\right|$. We recall (4.3) and write

$$
0 \leq G(u, l)-G_{L}(u, l) \leq A+B+C,
$$

with

$$
A=\left|G(u, l)-G\left(u^{\prime}, l^{\prime}\right)\right|, B=\left|G\left(u^{\prime}, l^{\prime}\right)-G_{L^{\prime}}\left(u^{\prime}, l^{\prime}\right)\right|, C=G_{L^{\prime}}\left(u^{\prime}, l^{\prime}\right)-G_{L}(u, l) .
$$

Since $G$ is $c$-Lipschitz on $\mathcal{H}_{\eta, M}$, and since $\left|(u, l)-\left(u^{\prime}, l^{\prime}\right)\right|_{\infty} \leq \varepsilon$, we have $A \leq c \varepsilon$. By (A.17) we have that $B \leq \varepsilon$. Therefore only $C$ remains to be considered. By Euclidean division, we get that $L=s L^{\prime}+r$, where $s \geq q$ and $r \in\left\{0, \ldots, L^{\prime}-1\right\}$. Pick $\pi_{1}, \pi_{2}, \ldots, \pi_{s} \in \mathcal{W}_{L^{\prime}}\left(u^{\prime},\left|l^{\prime}\right|\right)$, and concatenate them to obtain a trajectory in $\mathcal{W}_{s L^{\prime}}\left(u^{\prime},\left|l^{\prime}\right|\right)$. Moreover, note that

$$
\begin{aligned}
u L-u^{\prime} s L^{\prime} & =\left(u-u^{\prime}\right) s L^{\prime}+u r \\
& \geq\left(|l|-\left|l^{\prime}\right|\right) s L^{\prime}+(1+|l|) r=\left(L-s L^{\prime}\right)+\left(|l| L-s\left|l^{\prime}\right| L^{\prime}\right),
\end{aligned}
$$

where we use that $L-s L^{\prime}=r, u-u^{\prime} \geq|l|-\left|l^{\prime}\right|$ and $u \geq 1+|l|$. Thus, (A.20) implies that any trajectory in $\mathcal{W}_{L^{\prime}}\left(u^{\prime},\left|l^{\prime}\right|\right)$ can be concatenated with an $\left(u L-u^{\prime} s L^{\prime}\right)$-step trajectory, starting at $\left(s L^{\prime}, s\left|l^{\prime}\right| L^{\prime}\right)$ and ending at $(L,|l| L)$, to obtain a trajectory in $\mathcal{W}_{L}(u,|l|)$. Consequently,

$$
G_{L}(u, l) \geq \frac{s}{L} \log \kappa_{L^{\prime}}\left(u^{\prime}, l^{\prime}\right) \geq \frac{s}{s+1} G_{L^{\prime}}\left(u^{\prime}, l^{\prime}\right) .
$$

But $s \geq q$ and therefore $G_{L^{\prime}}\left(u^{\prime}, l^{\prime}\right)-G_{L}(u, l) \leq \frac{1}{q} G_{L^{\prime}}\left(u^{\prime}, l^{\prime}\right) \leq \frac{1}{q} M \log 3$ (recall that $\log 3$ is an upper bound for all entropies per step). Thus, by taking $q$ large enough, we complete the proof.

A.3.3. Proof of Lemma A.4. Pick $L \in \mathbb{N},(u, l) \in \mathcal{H}_{L}, \eta \in \frac{2 \mathbb{N}}{L}$, and define the map $T: \mathcal{W}_{L}(u, l) \mapsto \mathcal{W}_{L}(u+\eta, l)$ as follows. Pick $\pi \in \mathcal{W}_{L}(u, l)$, find its first vertical stretch, and extend this stretch by $\frac{\eta L}{2}$ steps. Then, find the first vertical stretch in the opposite direction of the stretch just extended, and extend this stretch by $\frac{\eta L}{2}$ steps. The result of this map is $T(\pi) \in \mathcal{W}_{L}(u+\eta, l)$, and it is easy to verify that $T$ is an injection, so that $\left|\mathcal{W}_{L}(u, l)\right| \leq\left|\mathcal{W}_{L}(u+\eta, l)\right|$.

Next, define a map $\widetilde{T}: \mathcal{W}_{L}(u+\eta, l) \mapsto \mathcal{W}_{L}(u, l)$ as follows. Pick $\pi \in \mathcal{W}_{L}(u+\eta, l)$ and remove its first $\frac{\eta L}{2}$ steps north and its first $\frac{\eta L}{2}$ steps south. The result is $\widetilde{T}(\pi) \in \mathcal{W}_{L}(u, l)$, but $\widetilde{T}$ is not injective. However, we can easily prove that for every $\varepsilon>0$ there exist $\eta_{\varepsilon}>0$ and $L_{\varepsilon} \in \mathbb{N}$ such that, for all $\eta<\eta_{\varepsilon}$ and all $L \geq l_{\varepsilon}$, the number of trajectories in $\mathcal{W}_{L}(u+\eta, l)$ that are mapped by $\widetilde{T}$ to a particular trajectory in $\pi \in \mathcal{W}_{L}(u, l)$ is bounded from above by $e^{\varepsilon L}$, uniformly in $(u, l) \in \mathcal{H}_{L}$ and $\pi \in \mathcal{W}_{L},(u, l)$.

This completes the proof of Lemmas A.2-A.4.

\section{Appendix B. Entropic properties}

Recall Lemma 4.1, where $(u, l) \mapsto \tilde{\kappa}(u, l)$ is defined on $\mathcal{H} \cap \mathbb{Q}^{2}$. 
Lemma B.1. (i) $(u, l) \mapsto u \tilde{\kappa}(u, l)$ extends to a continuous and strictly concave function on $\mathcal{H}$.

(ii) For all $u \in[1, \infty), l \mapsto \tilde{\kappa}(u, l)$ is strictly increasing on $[-u+1,0]$ and strictly decreasing on $[0, u-1]$.

(iii) For all $l \in \mathbb{R}, \lim _{u \rightarrow \infty} \tilde{\kappa}(u, l)=0$.

(iv) $\lim _{|l| \rightarrow \infty} \tilde{\kappa}(u, l)=0$ uniformly in $u \geq 1+|l|$.

(v) For all $l \in \mathbb{R}, u \mapsto u \tilde{\kappa}(u, l)$ is continuous, strictly concave, strictly increasing on $[1+|l|, \infty)$ and $\lim _{u \rightarrow \infty} u \tilde{\kappa}(u, l)=\infty$.

(vi) For all $l \in \mathbb{R}, u \mapsto u \tilde{\kappa}(u, l)$ is analytic on $(1+|l|, \infty)$ and

$$
\begin{aligned}
\lim _{v \rightarrow \infty} \partial_{u}(u \tilde{\kappa}(u, l))(v) & =0, \\
\lim _{v \rightarrow 1+l} \partial_{u}(u \tilde{\kappa}(u, l))(v) & =\partial_{u}^{+}(u \tilde{\kappa}(u, l))(1+|l|)=\infty .
\end{aligned}
$$

Lemma B.2. For all $\varepsilon>0$ there exists $R_{\varepsilon}>0$ such that

$$
\partial_{u}(u \tilde{\kappa}(u, l))(v) \leq \varepsilon, \quad \text { for } l \in[0, \infty), v \geq R_{\varepsilon} \vee 2+l .
$$

Recall the definition of $\{v(c), c \in(0, \infty)\}$ in $(3.8-3.10)$.

Lemma B.3. (i) For all $c \in(0, \infty), v(c) \in \overline{\mathcal{B}}$.

(ii) For $(k, l) \in\{A, B\} \times(0, \infty), c \mapsto v_{k, l}(c)$ is strictly decreasing and $c \mapsto v_{\mathcal{I}}(c)$ is non-increasing.

(iii) If $\left(c_{n}\right)_{n \in \mathbb{N}} \in(0, \infty)^{\mathbb{N}}$ satisfies $\lim _{n \rightarrow \infty} c_{n}=c_{\infty} \in(0, \infty)$, then $v\left(c_{n}\right)$ converges pointwise to $v\left(c_{\infty}\right)$.

(iv) $D(\bar{\rho}, v(c))<\infty$ for all $\bar{\rho} \in \mathcal{M}_{1}\left(\mathbb{R}_{+} \cup \mathbb{R}_{+} \cup\{\mathcal{I}\}\right)$ satisfying $\int_{0}^{\infty}(1+l)\left(\bar{\rho}_{A}+\right.$ $\left.\bar{\rho}_{B}\right)(d l)<\infty$ and all $c \in(0, \infty)$.

Recall the definition of $\{u(c), c \in(0, \infty)\}$ in (7.9).

Lemma B.4. (i) For all $c \in(0, \infty), u(c) \in \mathcal{B}_{\overline{\mathcal{V}}_{M}}$.

(ii) For all $\Theta \in \overline{\mathcal{V}}_{M}, c \mapsto u_{\Theta}$ (c) is non-increasing on $(0, \infty)$.

(iii) If $\left(c_{n}\right)_{n \in \mathbb{N}} \in(0, \infty)^{\mathbb{N}}$ satisfies $\lim _{n \rightarrow \infty} c_{n}=c_{\infty} \in(0, \infty)$, then $u\left(c_{n}\right)$ converges pointwise to $u\left(c_{\infty}\right)$.

(iv) $D(\rho, u(c))<\infty$ for all $\rho \in \mathcal{M}_{1}\left(\overline{\mathcal{V}}_{M}\right)$ satisfying $\int_{\overline{\mathcal{V}}_{M}} t_{\Theta} \rho(d \Theta)<\infty$ and all $c \in(0, \infty)$.

\section{B.1. Proofs of Lemmas B.1-B.4.}

B.1.1. Proof of Lemma B.1. (i) In the proof of Lemma A.1 we have shown that $\tilde{\kappa}$ can be extended to $\mathcal{H}^{0}$ in such a way that $(u, l) \mapsto u \tilde{\kappa}(u, l)$ is continuous and concave on $\mathcal{H}^{0}$. Lemma A.4 allows us to extend $\tilde{\kappa}$ to the boundary of $\mathcal{H}$, in such a way that continuity and concavity of $(u, l) \mapsto u \tilde{\kappa}(u, l)$ hold on all of $\mathcal{H}$. To obtain the strict concavity, we recall the formula in (4.4), i.e.,

$$
u \tilde{\kappa}(u, l)= \begin{cases}u \kappa(u /|l|, 1 /|l|), & l \neq 0, \\ u \hat{\kappa}(u), & l=0,\end{cases}
$$

where $(a, b) \mapsto a \kappa(a, b), a \geq 1+b, b \geq 0$, and $\mu \mapsto \mu \hat{\kappa}(\mu), \mu \geq 1$, are given in den Hollander and Whittington (2006), Section 2.1, and are strictly concave. In the case $l \neq 0,(B .4)$ provides strict concavity of $(u, l) \mapsto u \tilde{\kappa}(u, l)$ on $\mathcal{H}^{+}=\{(u, l) \in$ $\mathcal{H}: l>0\}$ and on $\mathcal{H}^{-}=\{(u, l) \in \mathcal{H}: l<0\}$, while in the case $l=0$ it provides strict concavity on $\overline{\mathcal{H}}=\{(u, 0), u \geq 1\}$. We already know that $(u, l) \mapsto u \tilde{\kappa}(u, l)$ 
is concave on $\mathcal{H}$, which, by the strict concavity on $\mathcal{H}^{+}, \mathcal{H}^{-}$and $\overline{\mathcal{H}}$, implies strict concavity of $(u, l) \mapsto u \tilde{\kappa}(u, l)$ on $\mathcal{H}$.

(ii) This follows from the strict concavity of $l \mapsto \tilde{\kappa}(u, l)$ and from the fact that $\tilde{\kappa}(u, l)=\tilde{\kappa}(u,-l)$ for $(u, l) \in \mathcal{H}$.

(iii-iv) These are direct consequences of Lemma A.2.

(v) By (i) we have that $u \mapsto u \tilde{\kappa}(u, l)$ is continuous and strictly concave on $[1+$ $|l|, \infty)$. Therefore, proving that $\lim _{u \rightarrow \infty} u \tilde{\kappa}(u, l)=\infty$ is sufficient to obtain that $u \mapsto u \tilde{\kappa}(u, l)$ is strictly increasing. It is proven in den Hollander and Whittington (2006), Lemma 2.1 .2 (iii), that $\lim _{\mu \rightarrow \infty} u \hat{\kappa}(u)=\infty$, so that (B.4) completes the proof for $l=0$. If $l \neq 0$, then we use (B.4) again and the variational formula in the proof of den Hollander and Whittington (2006), Lemma 2.1.1, to check that $\lim _{a \rightarrow \infty} a \kappa(a, b)=\infty$ for all $b>0$.

(vi) To get the analyticity on $(1+|l|, \infty)$, we use (B.4) and the analyticity of $(a, b) \mapsto a \kappa(a, b)$ and $\mu \mapsto \mu \hat{\kappa}(\mu)$ inside their domain of definition (see den Hollander and Whittington, 2006, Section 2.1).

We note that for every $l \in \mathbb{R}$,

$$
u \phi_{\mathcal{I}}(u) \geq u \tilde{\kappa}(u, 0) \geq u \tilde{\kappa}(u, l), \quad u \in[1+|l|, \infty),
$$

where the first inequality is well known and the second inequality comes from Lemma B.1(ii). Since, by Lemma B.1(v), $u \mapsto u \tilde{\kappa}(u, l)$ is concave and increasing on $[1+|l|, \infty),($ C.1) and (6.156) imply (B.1).

It remains to prove (B.2). To that aim, we recall that an explicit formula is available for $\tilde{\kappa}(u, l)$, namely,

$$
\tilde{\kappa}(u, l)=\kappa(u /|l|, 1 /|l|), \quad \text { for } l \neq 0,
$$

where $\kappa(a, b), a \geq 1+b, b \geq 0$ is given in den Hollander and Whittington (2006), Section 2.1 (in the proof of Lemmas 2.1.1-2.1.2). The latter formula allows us to compute $\partial_{u}(u \tilde{\kappa}(u, l))(1+l+\varepsilon, l)=G\left(1+\frac{1}{l}+\frac{\varepsilon}{l}, \frac{1}{l}\right)$ with

$$
G(a, b)=\frac{1}{2} \log \left[\frac{(a+1-b)(a-1-b)}{\left(a+1-b-2 \delta_{a, b}\right)\left(a-1-b-2 \varepsilon_{a, b}\right)}\right]
$$

and with

$$
\begin{aligned}
& \delta_{a, b}=\frac{b}{2(1+b)}\left[(a+1)-\left((a-b)^{2}+\left(b^{2}-1\right)\right)^{1 / 2}\right] \\
& \varepsilon_{a, b}=\frac{b}{2(1-b)}\left[-(a-1)+\left((a-b)^{2}+b^{2}-1\right)^{1 / 2}\right],
\end{aligned}
$$

so that the proof of (B.2) will be complete once we show that for all $b>0$ it holds that $\lim _{\varepsilon \rightarrow 0^{+}} G(1+b+\varepsilon, b)=\infty$. The latter is achieved by using first (B.8) to check that $\delta_{1+b+\varepsilon, b}=\frac{b}{1+b}+\left(\frac{1}{2}-\frac{1}{1+b}\right) \varepsilon+o(\varepsilon)$ and $\varepsilon_{1+b+\varepsilon, b}=\frac{\varepsilon}{2}+o(\varepsilon)$ as $\varepsilon \rightarrow 0^{+}$, and then by substituting these two expansions into (B.7) at $(a, b)=(1+b+\varepsilon, b)$, which implies the result after a straightforward computation.

B.1.2. Proof of Lemma B.2. The proof is based on the following lemma.

Lemma B.5.

$$
\lim _{l \rightarrow \infty} \partial_{u}[u \tilde{\kappa}(u, l)](2+l, l)=0 .
$$


Proof: We recall (B.6-B.8), and we note that $\partial_{u}(u \tilde{\kappa}(u, l))(2+l, l)=G\left(1+\frac{2}{l}, \frac{1}{l}\right)$. Thus, the proof of Lemma B.5 will be complete once we show that $\lim _{b \rightarrow 0^{+}} G(1+$ $2 b, b)=0$. The latter is achieved by using (B.7) and (B.8) to compute

$$
G(1+2 b, b)=\frac{1}{2} \log \left[\frac{(2+b) b}{\left[2+b\left(1-\frac{2}{1+b}+o(b)\right)\right](b+o(b))}\right]
$$

which immediately implies the result.

We resume the proof of Lemma B.2. Once Lemma B.5 is proven, we use the concavity of $u \mapsto u \tilde{\kappa}(u, l)$ for $l \in \mathbb{R}$ to obtain that for $\varepsilon>0$ there exists a $l_{\varepsilon}>0$ such that $\partial_{u}[u \tilde{\kappa}(u, l)](u, l) \leq \varepsilon$ for all $l:|l| \geq l_{\varepsilon}$ and $u \geq 2+l$. Thus, it remains to show that there exists a $R_{\varepsilon}>0$ such that $\partial_{u}[u \tilde{\kappa}(u, l)](u, l) \leq \varepsilon$ for $l \in\left[0, l_{\varepsilon}\right]$ and $u \geq R_{\varepsilon}$. By contradiction, if we assume that the latter does not hold, then there exists $\varepsilon>0$ and two sequences $\left(l_{n}\right)_{n \in \mathbb{N}} \in\left[0, l_{\varepsilon}\right]^{\mathbb{N}}$ and $\left(u_{n}\right)_{n \in \mathbb{N}}$ such that $u_{n} \geq 1+l_{n}$ for $n \in \mathbb{N}$ and $\lim _{n \rightarrow \infty} u_{n}=\infty$ and such that $\partial_{u}[u \tilde{\kappa}(u, l)]\left(u_{n}, l_{n}\right) \geq \varepsilon$ for $n \in \mathbb{N}$. As a consequence, we can write

$$
u_{n} \tilde{\kappa}\left(u_{n}, l_{n}\right)-\left(1+l_{n}\right) \tilde{\kappa}\left(1+l_{n}, l_{n}\right) \geq \varepsilon\left(u_{n}-1-l_{n}\right),
$$

and, with the help of Lemma B.1(ii), we obtain

$$
u_{n} \tilde{\kappa}\left(u_{n}, 0\right) \geq u_{n} \tilde{\kappa}\left(u_{n}, l_{n}\right) \geq \varepsilon\left(u_{n}-1-l_{\varepsilon}\right), \quad \text { for } n \in \mathbb{N},
$$

which clearly contradicts Lemma B.1(iii) because $\lim _{n \rightarrow \infty} u_{n}=\infty$.

B.1.3. Proof of Lemma B.3. (i) We must prove that $l \mapsto v_{A, l}(c)$ and $l \mapsto v_{B, l}(c)$ are continuous on $[0, \infty)$. We give the proof for $v_{A}$, the proof for $v_{B}$ being similar. Let $\left(l_{n}\right)_{n \in \mathbb{N}}$ be a sequence in $[0, \infty)$ such that $\lim _{n \rightarrow \infty} l_{n}=l_{\infty} \in[0, \infty)$. We want to prove that $\lim _{n \rightarrow \infty} v_{A, l_{n}}(c)=v_{A, l_{\infty}}(c)$. For simplicity, we set $v_{n}=v_{A, l_{n}}(c)$ for $n \in \mathbb{N}$ and $v_{\infty}=v_{A, l_{\infty}}(c)$. We also set $g_{n}(u)=u \tilde{\kappa}\left(u, l_{n}\right)$ for $n \in \mathbb{N}$ and $u \geq 1+l_{n}$ and $g_{\infty}(u)=u \tilde{\kappa}\left(u, l_{\infty}\right)$ for $u \geq 1+l_{\infty}$. By Lemmas B.1(i) and (v), we know that $g_{n}$ converges pointwise to $g_{\infty}$ as $n \rightarrow \infty$, and that $g_{n}$ and $g_{\infty}$ are strictly concave. Consequently, $\partial_{u}\left(g_{n}\right)$ converges pointwise to $\partial_{u}\left(g_{\infty}\right)$. We argue by contradiction. Suppose that $v_{n}$ does not converge to $v_{\infty}$. Then there exists an $\eta>0$ such that $v_{n} \geq v_{\infty}+\eta$ along a subsequence or $v_{n} \leq v_{\infty}-\eta$ along a subsequence. Suppose for simplicity that $v_{n} \leq v_{\infty}-\eta$ for $n \in \mathbb{N}$. Then the strict concavity of $g_{n}$ implies that $\partial_{u}\left(g_{n}\right)\left(v_{\infty}-\eta\right) \leq \partial_{u}\left(g_{n}\right)\left(v_{n}\right)=c$, and therefore, letting $n \rightarrow \infty$ and using the strict concavity of $g_{\infty}$, we obtain $\partial_{u}\left(g_{\infty}\right)\left(v_{\infty}\right)<\partial_{u}\left(g_{\infty}\right)\left(v_{\infty}-\eta\right) \leq c$. This provides the contradiction, because $\partial_{u}\left(g_{\infty}\right)\left(v_{\infty}\right)=c$ by definition. The proof is similar when we assume that $v_{n} \geq v_{\infty}+\eta$ for $n \in \mathbb{N}$.

(ii) For $(k, l) \in\{A, B\} \times[0, \infty)$, this is a straightforward consequence of the definition of $v(c)$ in (3.8-3.9), of the strict concavity of $u \mapsto u \tilde{\kappa}(u, l)$ and of the continuity of $u \mapsto \partial_{u}(u \tilde{\kappa}(u, l))$ for every $l \in[0, \infty)$ (see Lemma B.1(v-vi)). For $c \mapsto v_{\mathcal{I}}(c)$ we do not have strict monotonicity because $u \mapsto \partial_{u}\left(u \phi_{\mathcal{I}}(u)\right)$ is not proven to be continuous.

(iii) Similarly to what we did in (i), we consider $\left(c_{n}\right)_{n \in \mathbb{N}}$ a sequence in $(0, \infty)$ such that $\lim _{n \rightarrow \infty} c_{n}=c_{\infty} \in(0, \infty)$, and we want to show that $\lim _{n \rightarrow \infty} v_{k, l}\left(c_{n}\right)=$ $v_{k, l}\left(c_{\infty}\right)$ for $k \in\{A, B\}$ and $l \in[0, \infty)$ and $\lim _{n \rightarrow \infty} v_{\mathcal{I}}\left(c_{n}\right)=v_{\mathcal{I}}\left(c_{\infty}\right)$. Again we argue by contradiction. Suppose, for instance, that $v_{\mathcal{I}}\left(c_{n}\right)$ does not converge to $v_{\mathcal{I}}\left(c_{\infty}\right)$. Then there exists an $\eta>0$ such that $v_{\mathcal{I}}\left(c_{n}\right) \leq v_{\mathcal{I}}\left(c_{\infty}\right)-\eta$ or $v_{\mathcal{I}}\left(c_{n}\right) \geq$ $v_{\mathcal{I}}\left(c_{\infty}\right)+\eta$ along a subsequence. Suppose for simplicity that $v_{\mathcal{I}}\left(c_{n}\right) \geq v_{\mathcal{I}}\left(c_{\infty}\right)+\eta$. 
Then $\partial_{u}^{-}\left(u \phi_{\mathcal{I}}(u)\right)\left(v_{\mathcal{I}}\left(c_{\infty}\right)+\eta\right) \geq \partial_{u}^{-}\left(u \phi_{\mathcal{I}}(u)\right)\left(v_{\mathcal{I}}\left(c_{n}\right)\right) \geq c_{n}$ for $n \in \mathbb{N}$. Let $n \rightarrow \infty$ to obtain $\partial_{u}^{+}\left(u \phi_{\mathcal{I}}(u)\right)\left(v_{\mathcal{I}}\left(c_{\infty}\right)\right)>\partial_{u}^{-}\left(u \phi_{\mathcal{I}}(u)\right)\left(v_{\mathcal{I}}\left(c_{\infty}\right)+\eta\right) \geq c_{\infty}$, which contradicts the definition of $v_{\mathcal{I}}\left(c_{\infty}\right)$ in $(3.8-3.10)$. The proof is similar when we assume that $v_{\mathcal{I}}\left(c_{n}\right) \leq v_{\mathcal{I}}\left(c_{\infty}\right)-\eta$ for $n \in \mathbb{N}$.

(iv) This is a consequence of Lemma B.2, which implies that for all $c \in(0, \infty)$ there exists a $l_{c} \in[0, \infty)$ such that $v_{A, l}(c) \leq 2+l$ for all $l \geq l_{c}$. Moreover, (3.8-3.9) and the fact that $(\alpha, \beta) \in \mathrm{CONE}$ entail that $v_{B, l}(c) \leq v_{A, l}(c)$ for $l \in[0, \infty)$, and therefore $\int_{0}^{\infty}(1+l)\left(\bar{\rho}_{A}+\bar{\rho}_{B}\right)(d l)<\infty$ combined with the finitness of $v_{\mathcal{I}}(c)$ imply $\bar{D}(\bar{\rho}, v(c))<\infty$.

B.1.4. Proof of Lemma B.4. (i) The proof is similar to that of Lemma B.3(i), except for the fact that when we consider $\Theta_{n} \rightarrow \Theta_{\infty}$ as $n \rightarrow \infty$ in $\overline{\mathcal{V}}_{M}$, we have (by Lemma C.3) the pointwise convergence of $g_{n}(u)=u \psi\left(\Theta_{n}, u\right)$ to $g_{\infty}(u)=u \psi\left(\Theta_{\infty}, u\right)$, but we do not have the pointwise convergence of $\partial g_{n}(u)$ to $\partial g_{\infty}(u)$ since $g_{\infty}$ is not a priori differentiable. However, the strict concavity and the pointwise convergence of $g_{n}$ towards $g_{\infty}$ gives us

$$
\partial^{-} g_{\infty}(u) \geq \limsup _{n \rightarrow \infty} \partial^{-} g_{n}(u) \geq \liminf _{n \rightarrow \infty} \partial^{+} g_{n}(u) \geq \partial^{+} g_{\infty}(u),
$$

with which we can easily mimic the proof in Lemma B.3(i)

(ii) The proof is similar to that of Lemma B.3(ii), except for the fact that the monotonicity of $c \mapsto u_{\Theta}(c)$ is not proven to be strict because $u \mapsto \partial(u \psi(\Theta, u))$ is not proven to be continuous.

(iii) We mimic the proof of Lemma B.3(iii). Let $\left(c_{n}\right)_{n \in \mathbb{N}}$ be a sequence in $(0, \infty)$ such that $\lim _{n \rightarrow \infty} c_{n}=c_{\infty} \in(0, \infty)$, and assume that there exists an $\eta>0$ such that $u_{\Theta}\left(c_{n}\right) \geq u_{\Theta}\left(c_{\infty}\right)+\eta$ along a subsequence. Then $\partial_{u}^{-}(u \psi(\Theta, u))\left(u_{\Theta}\left(c_{\infty}\right)+\eta\right) \geq$ $\partial_{u}^{-}(u \psi(\Theta, u))\left(u_{\Theta}\left(c_{n}\right)\right) \geq c_{n}$ for $n \in \mathbb{N}$. Let $n \rightarrow \infty$ to obtain $\partial_{u}^{+}(u \psi(\Theta, u))\left(u_{\Theta}\left(c_{\infty}\right)\right)$ $>\partial_{u}^{-}(u \psi(\Theta, u))\left(u_{\Theta}\left(c_{\infty}\right)+\eta\right) \geq c_{\infty}$, which contradicts the definition of $u_{\Theta}\left(c_{\infty}\right)$ in (7.9).

(iv) The proof is similar to that of Lemma B.3(iv). The role of Lemma B.2 is taken over by Lemma C.8

\section{Appendix C. Properties of free energies}

C.1. Free energy along a single linear interface. Also the free energy $\mu \mapsto \phi^{\mathcal{I}}(\mu ; \alpha, \beta)$ defined in Proposition 4.2 can be extended from $\mathbb{Q} \cap[1, \infty)$ to $[1, \infty)$, in such a way that $\mu \mapsto \mu \phi^{\mathcal{I}}(\mu ; \alpha, \beta)$ is concave and continous on $[1, \infty)$. By concatenating trajectories, we can indeed check that $\mu \mapsto \mu \phi^{\mathcal{I}}(\mu ; \alpha, \beta)$ is concave on $\mathbb{Q} \cap[1, \infty)$. Therefore it is Lipschitz on every compact subset of $(1, \infty)$ and can be extended to a concave and continuous function on $(1, \infty)$. The continuity at $\mu=1$ comes from the fact that $\phi^{\mathcal{I}}(1 ; \alpha, \beta)=0$ and $\lim _{\mu \downarrow 1} \phi^{\mathcal{I}}(\mu)=0$, which is obtained by using Lemma E.1 below.

Lemma C.1. For all $(\alpha, \beta) \in \mathrm{CONE}$ :

(i) $\mu \mapsto \mu \phi^{\mathcal{I}}(\mu ; \alpha, \beta)$ is strictly increasing on $[1, \infty)$ and $\lim _{\mu \rightarrow \infty} \mu \phi^{\mathcal{I}}(\mu ; \alpha, \beta)=\infty$.

(ii) $\lim _{\mu \rightarrow \infty} \phi^{\mathcal{I}}(\mu ; \alpha, \beta)=0$. 
(iii)

$$
\begin{aligned}
\lim _{v \rightarrow \infty} \partial_{u}^{-}\left(u \phi_{\mathcal{I}}(u ; \alpha, \beta)\right)(v) & =0, \\
\lim _{v \rightarrow 1} \partial_{u}^{+}\left(u \phi_{\mathcal{I}}(u ; \alpha, \beta)\right)(v) & =\partial_{u}^{+}\left(u \phi_{\mathcal{I}}(u ; \alpha, \beta)\right)(1)=\infty .
\end{aligned}
$$

Proof: (i) Clearly, $\phi^{\mathcal{I}}(\mu ; \alpha, \beta) \geq \widetilde{\kappa}(\mu, 0)$ for $\mu \geq 1$. Therefore Lemma B.1(iv) implies that $\lim _{\mu \rightarrow \infty} \mu \phi^{\mathcal{I}}(\mu ; \alpha, \beta)=\infty$. Thus, the concavity of $\mu \mapsto \mu \phi^{\mathcal{I}}(\mu ; \alpha, \beta)$ is sufficient to obtain that it is strictly increasing on $[1, \infty)$.

(ii) See den Hollander and Pétrélis (2009b), Lemma 2.4.1(i).

(iii) To prove (C.1), we pick $\chi \in\{A, B\}^{\mathbb{Z}}$ such that $\chi(0)=A$ and $\chi(-1)=B$. We recall (4.40) and consider $\Theta=(\chi, 0,0,0,2) \in \overline{\mathcal{V}}_{\text {nint }, A, 2, M}$ such that $l_{A}(\Theta)=$ $l_{B}(\Theta)=0$. By Proposition 4.6, we have

$$
u \psi\left(\Theta_{2}, u\right) \geq u \phi_{\mathcal{I}}(u), \quad u \in[1, \infty),
$$

and (C.3), together with Lemma C.7 and the concavity and monotonicity of $u \mapsto$ $u \phi_{\mathcal{I}}(u)$, imply (C.1).

It remains to prove (C.2). For all $(\alpha, \beta) \in$ CONE we know that $u \mapsto u \phi_{\mathcal{I}}(u ; \alpha, \beta)$ is continuous and strictly concave on $[1, \infty)$. Therefore we necessarily have

$$
\lim _{v \rightarrow 1^{+}} \partial_{u}^{+}\left(u \phi_{\mathcal{I}}(u)\right)(v)=\partial_{u}^{+}\left(u \phi_{\mathcal{I}}(u)\right)(1) .
$$

Moreover, since $\left(u \phi_{\mathcal{I}}(u)\right)(1)=(u \tilde{\kappa}(u, 0))(1)=0$ and since $\phi_{\mathcal{I}}(u) \geq \tilde{\kappa}(u, 0)$ for $u \geq$ 1 , we have $\partial_{u}^{+}\left(u \phi_{\mathcal{I}}(u)\right)(1) \geq \partial_{u}^{+}(u \tilde{\kappa}(u, 0))(1)$ and (B.2) gives $\partial_{u}^{+}(u \tilde{\kappa}(u, 0))(1)=\infty$, which completes the proof of (C.2).

Recall Assumption 4.3, in which we assumed that $\mu \mapsto \mu \phi^{\mathcal{I}}(\mu ; \alpha, \beta)$ is strictly concave on $[1, \infty)$. The next lemma states that the convergence of the average quenched free energy $\phi_{L}^{\mathcal{I}}$ to $\phi^{\mathcal{I}}$ as $L \rightarrow \infty$ is uniform on $\mathbb{Q} \cap[1, \infty)$.

Lemma C.2. For every $(\alpha, \beta) \in \mathrm{CONE}$ and $\varepsilon>0$ there exists an $L_{\varepsilon} \in \mathbb{N}$ such that

$$
\left|\phi_{L}(\mu)-\phi(\mu)\right| \leq \varepsilon \quad \forall \mu \in 1+\frac{2 \mathbb{N}}{L}, L \geq L_{\varepsilon} .
$$

Proof: Similarly to what we did for Lemma A.1, the proof can be done by treating separately the cases $\mu$ large, moderate and small. We leave the details to the reader.

C.2. Free energy in a single column. We can extend $(\Theta, u) \mapsto \psi(\Theta, u)$ from $\mathcal{V}_{M}^{*}$ to $\overline{\mathcal{V}}_{M}^{*}$ by using the variational formula in (4.48) and by recalling that $\widetilde{\kappa}$ and $\phi^{\mathcal{I}}$ have been extended to $\mathcal{H}$ and $[1, \infty)$ in Appendices A.3 and C.1.

Pick $M \in \mathbb{N}$ and recall (4.18). Define a distance $d_{M}$ on $\overline{\mathcal{V}}_{M}$ as follows. Pick $\Theta_{1}, \Theta_{2} \in \overline{\mathcal{V}}_{M}$, abbreviate

$$
\Theta_{1}=\left(\chi_{1}, \Delta \Pi_{1}, b_{0,1}, b_{1,1}, x_{1}\right), \quad \Theta_{2}=\left(\chi_{2}, \Delta \Pi_{2}, b_{0,2}, b_{1,2}, x_{2}\right),
$$

and define

$d_{M}\left(\Theta_{1}, \Theta_{2}\right)=\sum_{j \in \mathbb{Z}} \frac{1_{\left\{\chi_{1}(j) \neq \chi_{2}(j)\right\}}}{2^{|j|}}+\left|\Delta \Pi_{1}-\Delta \Pi_{2}\right|+\left|b_{0,1}-b_{0,2}\right|+\left|b_{1,1}-b_{1,2}\right|+\left|x_{1}-x_{2}\right|$

so that $\widetilde{d}_{M}\left(\left(\Theta_{1}, u_{1}\right),\left(\Theta_{2}, u_{2}\right)\right)=\max \left\{\left|u_{1}-u_{2}\right|, d_{M}\left(\Theta_{1}, \Theta_{2}\right)\right\}$ is a distance on $\overline{\mathcal{V}}_{M}^{*, m}$ for which $\overline{\mathcal{V}}_{M}^{*, m}$ is compact.

Lemmas C.3 and C.4 below are proven in Section C.3. 
Lemma C.3. For every $(M, m) \in \mathrm{EIGH}$ and $(\alpha, \beta) \in \mathrm{CONE}$,

$$
(u, \Theta) \mapsto u \psi(\Theta, u ; \alpha, \beta)
$$

is uniformly continuous on $\overline{\mathcal{V}}_{M}^{*, m}$ endowed with $\widetilde{d}_{M}$.

Lemma C.4. For every $\Theta \in \overline{\mathcal{V}}_{M}$, the function $u \mapsto u \psi(\Theta, u)$ is continuous and strictly concave on $\left[t_{\Theta}, \infty\right)$.

Below we list several results that were used in Section 7. The proofs of these result are given in Section C.3. Proposition C.5 below says that the free energy per column associated with the Hamiltonian given by $(\beta-\alpha) / 2$ times the time spent by the copolymer in the $B$-solvent is a good aproximation of $\psi(\Theta, u)$ when $u \rightarrow \infty$ uniformly in $\Theta \in \overline{\mathcal{V}}_{M}$. This proof of this proposition will be given in Section C.3.3.

Proposition C.5. For all $(\alpha, \beta) \in \mathrm{CONE}$ and all $\varepsilon>0$ there exists $R_{\varepsilon}>0$ and $L_{\varepsilon} \in \mathbb{N}$ such that

$$
\left|\psi(\Theta, u)-\frac{1}{u L} \log \sum_{\pi \in \mathcal{W}_{\Theta, u, L}} e^{T(\pi) \frac{\beta-\alpha}{2}}\right| \leq \varepsilon, \quad \Theta \in \overline{\mathcal{V}}_{M}, \quad u \geq t_{\Theta} \vee R_{\varepsilon}, \quad L \geq L_{\varepsilon},
$$

where $T(\pi)=\sum_{i=1}^{u L} 1\left\{\chi_{\left(\pi_{i-1}, \pi_{i}\right)}^{L}=B\right\}$ is the time spent by $\pi$ in solvent $B$.

Lemmas C.6-C.8 below are consequences of Lemma C.4 and Proposition C.5. The proofs of Lemmas C.6 and C.8 will be given in Sections C.3.4 and C.3.6. Lemma C.6 shows that $\psi(\Theta, u)$ is bounded from above uniformly in $\Theta \in \overline{\mathcal{V}}_{M}$ as $u \rightarrow \infty$. Lemma C.7 identifies the limit of $\partial_{u}^{-}(u \psi(\Theta, u))$ as $u \rightarrow \infty$ for $\Theta \in \overline{\mathcal{V}}_{M}$. Lemma C.8 is the counterpart of Lemma C.6 for $\partial_{u}^{-}(u \psi(\Theta, u))$ instead of $\psi(\Theta, u)$.

Lemma C.6. For all $(\alpha, \beta) \in \mathrm{CONE}$ and $\varepsilon>0$ there exists a $C_{\varepsilon}>0$ such that

$$
\psi(\Theta, u) \leq \begin{cases}\varepsilon & \text { if } \Theta \in \overline{\mathcal{V}}_{M} \backslash \overline{\mathcal{V}}_{\text {nint }, B, 1, M}, \quad u \geq t_{\Theta} \vee C_{\varepsilon}, \\ \frac{\beta-\alpha}{2}+\varepsilon & \text { if } \Theta \in \overline{\mathcal{V}}_{\text {nint }, B, 1, M}, \quad u \geq t_{\Theta} \vee C_{\varepsilon},\end{cases}
$$

Lemma C.7. For all $(\alpha, \beta) \in \mathrm{CONE}$,

$$
\lim _{v \rightarrow \infty} \partial_{u}^{+}(u \psi(\Theta, u))(v)= \begin{cases}0 & \text { if } \Theta \in \overline{\mathcal{V}}_{M} \backslash \overline{\mathcal{V}}_{\text {nint }, B, 1, M}, \\ \frac{\beta-\alpha}{2} & \text { if } \Theta \in \overline{\mathcal{V}}_{\text {nint }, B, 1, M} .\end{cases}
$$

Lemma C.8. For all $(\alpha, \beta) \in \mathrm{CONE}$ and $\varepsilon>0$ there exists a $V_{\varepsilon}>0$ such that

$$
\partial_{u}^{-}(u \psi(\Theta, u))(v) \leq \begin{cases}\varepsilon & \text { if } \Theta \in \overline{\mathcal{V}}_{M} \backslash \overline{\mathcal{V}}_{\text {nint }, B, 1, M}, \quad v \geq 2 t_{\Theta} \vee V_{\varepsilon}, \\ \frac{\beta-\alpha}{2}+\varepsilon & \text { if } \Theta \in \overline{\mathcal{V}}_{\text {nint }, B, 1, M}, \quad v \geq 2 t_{\Theta} \vee V_{\varepsilon} .\end{cases}
$$

\section{C.3. Proof of Lemmas C.3-C.8.}

C.3.1. Proof of Lemma C.3. Pick $(M, m) \in$ EIGH. By the compactness of $\overline{\mathcal{V}}_{M}^{*, m}$, it suffices to show that $(u, \Theta) \mapsto u \psi(\Theta, u)$ is continuous on $\overline{\mathcal{V}}_{M}^{*, m}$. Let $\left(\Theta_{n}, u_{n}\right)=$ $\left(\chi_{n}, \Delta \Pi_{n}, b_{0, n}, b_{1, n}, u_{n}\right)$ be the general term of an infinite sequence that tends to $(\Theta, u)=\left(\chi, \Delta \Pi, b_{0}, b_{1}, u\right)$ in $\left(\overline{\mathcal{V}}_{M}^{*, m}, \widetilde{d}_{M}\right)$. We want to show that $\lim _{n \rightarrow \infty}$ $u_{n} \psi\left(\Theta_{n}, u_{n}\right)=u \psi(\Theta, u)$. By the definition of $\widetilde{d}_{M}$, we have $\chi_{n}=\chi$ and $\Delta \Pi_{n}=\Delta \Pi$ 
for $n$ large enough. We assume that $\Theta \in \mathcal{V}_{\text {int }}$, so that $\Theta_{n} \in \mathcal{V}_{\text {int }}$ for $n$ large enough as well. The case $\Theta \in \mathcal{V}_{\text {nint }}$ can be treated similarly.

Set

$$
\mathcal{R}_{m}=\{(a, h, l) \in[0, m] \times[0,1] \times \mathbb{R}: h+|l| \leq a\}
$$

and note that $\mathcal{R}_{m}$ is a compact set. Let $g: \mathcal{R}_{m} \mapsto[0, \infty)$ be defined as $g(a, h, l)=$ $a \widetilde{\kappa}\left(\frac{a}{h}, \frac{l}{h}\right)$ if $h>0$ and $g(a, h, l)=0$ if $h=0$. The continuity of $\widetilde{\kappa}$, stated in Lemma B.1(i), ensures that $g$ is continuous on $\left\{(a, h, l) \in \mathcal{R}_{m}: h>0\right\}$. The continuity at all $(a, 0, l) \in \mathcal{R}_{m}$ is obtained by recalling that $\lim _{u \rightarrow \infty} \tilde{\kappa}(u, l)=0$ uniformly in $l \in[-u+1, u-1]$ (see Lemma B.1(ii-iii)) and that $\widetilde{\kappa}$ is bounded on $\mathcal{H}$.

In the same spirit, we may set $\mathcal{R}_{m}^{\prime}=\{(u, h) \in[0, m] \times[0,1]: h \leq u\}$ and define $g^{\prime}: \mathcal{R}_{m}^{\prime} \mapsto[0, \infty)$ as $g^{\prime}(u, h)=u \phi^{\mathcal{I}}\left(\frac{u}{h}\right)$ for $h>0$ and $g^{\prime}(u, h)=0$ for $h=0$. With the help of Lemma C.1 we obtain the continuity of $g^{\prime}$ on $\mathcal{R}_{m}^{\prime}$ by mimicking the proof of the continuity of $g$ on $\mathcal{R}_{m}$.

Note that the variational formula in (4.48) can be rewriten as

$$
u \psi(\Theta, u)=\sup _{(h),(a) \in \mathcal{L}\left(l_{A}, l_{B} ; u\right)} Q\left((h),(a), l_{A}, l_{B}\right),
$$

with

$$
Q\left((h),(a), l_{A}, l_{B}\right)=g\left(a_{A}, h_{A}, l_{A}\right)+g\left(a_{B}, h_{B}, l_{B}\right)+a_{B} \frac{\beta-\alpha}{2}+g^{\prime}\left(a^{\mathcal{I}}, h^{\mathcal{I}}\right),
$$

and with $l_{A}$ and $l_{B}$ defined in (4.39). Note that $\mathcal{L}\left(l_{A}, l_{B} ; u\right)$ is compact, and that $(h),(a) \mapsto Q\left((h),(a), l_{A}, l_{B}\right)$ is continuous on $\mathcal{L}\left(l_{A}, l_{B} ; u\right)$ because $g$ and $g^{\prime}$ are continuous on $\mathcal{R}_{m}$ and $\mathcal{R}_{m}^{\prime}$, respectively. Hence, the supremum in (C.14) is attained.

Pick $\varepsilon>0$, and note that $g$ and $g^{\prime}$ are uniformly continuous on $\mathcal{R}_{m}$ and $\mathcal{R}_{m}^{\prime}$, which are compact sets. Hence there exists an $\eta_{\varepsilon}>0$ such that $\mid g(a, h, l)-$ $g\left(a^{\prime}, h^{\prime}, l^{\prime}\right) \mid \leq \varepsilon$ and $\left|g^{\prime}(u, b)-g^{\prime}\left(u^{\prime}, b^{\prime}\right)\right| \leq \varepsilon$ when $(a, h, l),\left(a^{\prime}, h^{\prime}, l^{\prime}\right) \in \mathcal{R}_{m}$ and $(u, b),\left(u^{\prime}, b^{\prime}\right) \in \mathcal{R}_{m}^{\prime}$ are such that $\left|a-a^{\prime}\right|,\left|h-h^{\prime}\right|,\left|l-l^{\prime}\right|,\left|u-u^{\prime}\right|$ and $\left|b-b^{\prime}\right|$ are bounded from above by $\eta_{\varepsilon}$.

Since $\lim _{n \rightarrow \infty}\left(\Theta_{n}, u_{n}\right)=(\Theta, u)$ we also have that $\lim _{n \rightarrow \infty} b_{0, n}=b_{0}$, $\lim _{n \rightarrow \infty} b_{1, n}=b_{1}$ and $\lim _{n \rightarrow \infty} u_{n}=u$. Thus, $\lim _{n \rightarrow \infty} l_{A, n}=l_{A}$ and $\lim _{n \rightarrow \infty} l_{B, n}=$ $l_{B}$, and therefore $\left|l_{A, n}-l_{A}\right| \leq \eta_{\varepsilon},\left|l_{B, n}-l_{B}\right| \leq \eta_{\varepsilon}$ and $\left|u_{n}-u\right| \leq \eta_{\varepsilon}$ for $n \geq n_{\varepsilon}$ large enough.

For $n \in \mathbb{N}$, let $\left(h_{n}\right),\left(a_{n}\right) \in \mathcal{L}\left(l_{A, n}, l_{B, n} ; u_{n}\right)$ be a maximizer of (C.14) at $\left(\Theta_{n}, u_{n}\right)$, and note that, for $n \geq n_{\varepsilon}$, we can choose $\left(\widetilde{h}_{n}\right),\left(\widetilde{a}_{n}\right) \in \mathcal{L}\left(l_{A}, l_{B} ; u\right)$ such that $\left|\widetilde{a}_{A, n}-a_{A, n}\right|,\left|\widetilde{a}_{B, n}-a_{B, n}\right|,\left|\widetilde{a}_{n}^{\mathcal{I}}-a_{n}^{\mathcal{I}}\right|,\left|\widetilde{h}_{A, n}-h_{A, n}\right|,\left|\widetilde{h}_{B, n}-h_{B, n}\right|$ and $\left|\widetilde{h}_{n}^{\mathcal{I}}-h_{n}^{\mathcal{I}}\right|$ are bounded above by $\eta_{\varepsilon}$. Consequently,

$$
u_{n} \psi\left(\Theta_{n}, u_{n}\right)-u \psi(\Theta, u) \leq Q\left(\left(h_{n}\right),\left(a_{n}\right), l_{A, n}, l_{B, n}\right)-Q\left(\left(\widetilde{h}_{n}\right),\left(\widetilde{a}_{n}\right), l_{A}, l_{B}\right) \leq 3 \varepsilon .
$$

We bound $u \psi(\Theta, u)-u_{n} \psi\left(\Theta_{n}, u_{n}\right)$ from above in a similar manner, and this suffices to obtain the claim.

C.3.2. Proof of lemma C.4. The continuity is a straightforward consequence of Lemma C.3: simply fix $\Theta$ and let $m \rightarrow \infty$. To prove the strict concavity, we note that the cases $\Theta \in \mathcal{V}_{\text {int }, M}$ and $\Theta \in \mathcal{V}_{\text {nint }, M}$ can be treated similarly. We will therefore focus on $\Theta \in \mathcal{V}_{\text {int }, M}$. 
For $l \in \mathbb{R}$, let

$$
\mathcal{N}_{l}=\{(a, h) \in[0, \infty) \times[0,1]: a \geq h+|l|\}, \quad \mathcal{N}_{l}^{+}=\left\{(a, h) \in \mathcal{N}_{l}: h>0\right\},
$$

and let $g_{l}: \mathcal{N}_{l} \mapsto[0, \infty)$ be defined as $g_{l}(a, h)=a \tilde{\kappa}\left(\frac{a}{h}, \frac{l}{h}\right)$ for $h>0$ and $g_{l}(a, h)=0$ for $h=0$. For $l \neq 0$, the strict concavity of $(u, l) \mapsto u \tilde{\kappa}(u, l)$ on $\mathcal{H}$, stated in Lemma B.1(i), immediately yields that $g_{l}$ is strictly concave on $\mathcal{N}_{l}^{+}$and concave on $\mathcal{N}_{l}$. Consequently, for all $\left(a_{1}, h_{1}\right) \in \mathcal{N}_{l}^{+}$and $\left(a_{2}, h_{2}\right) \in \mathcal{N}_{l} \backslash \mathcal{N}_{l}^{+}, g_{l}$ is strictly concave on the segment $\left[\left(u_{1}, h_{1}\right),\left(u_{2}, h_{2}\right)\right]$.

Define also $\widetilde{g}: \mathcal{N}_{0} \mapsto[0, \infty)$ as $\widetilde{g}(a, h)=a \phi^{\mathcal{I}}\left(\frac{a}{h}\right)$ for $h>0$ and $\widetilde{g}(a, h)=0$ for $h=0$. The strict concavity of $u \mapsto u \phi^{\mathcal{I}}(u)$ and of $u \mapsto u \tilde{\kappa}(u, 0)$ on $[1, \infty)$, stated in Assumption 4.3 and in Lemma B.1, immediately yield that $\widetilde{g}$ and $g_{0}$ are concave on $\mathcal{N}_{0}$ and that, for $h>0, a \mapsto \widetilde{g}(a, h)$ and $a \mapsto g_{0}(a, h)$ are strictly concave on $[h, \infty)$

Similarly to what we did in (C.14), we can rewrite the variational formula in (4.48) as

$$
u \psi(\Theta, u)=\sup _{(h),(a) \in \mathcal{L}\left(l_{A}, l_{B} ; u\right)} \widetilde{Q}((h),(a))
$$

with

$$
\widetilde{Q}((h),(a))=g_{l_{A}}\left(a_{A}, h_{A}\right)+g_{l_{B}}\left(a_{B}, h_{B}\right)+a_{B} \frac{\beta-\alpha}{2}+\widetilde{g}\left(u-a_{A}-a_{B}, 1-h_{A}-h_{B}\right),
$$

and the supremum in (C.18) is attained. In what follows we will restrict the proof to the case $l_{A}, l_{B}>0$ for the following reason. If $l_{k}=0$ for $k \in\{A, B\}$, then the inequality $g_{0} \leq \widetilde{g}$ and the concavity of $\widetilde{g}$ ensure that there exists a $(h),(a) \in$ $\mathcal{L}\left(l_{A}, l_{B} ; u\right)$ maximizing (C.18) and satisfying $h_{k}=a_{k}=0$, which allows to copy the proof below after removing the $k$-th coordinate in $(h),(a)$.

Next, we show that if $(h),(a) \in \mathcal{L}\left(l_{A}, l_{B} ; u\right)$ realizes the maximum in (C.18), then $(h),(a) \notin \widetilde{\mathcal{L}}\left(l_{A}, l_{B} ; u\right)$ with

$$
\widetilde{\mathcal{L}}\left(l_{A}, l_{B} ; u\right)=\widetilde{\mathcal{L}}_{A}\left(l_{A}, l_{B} ; u\right) \cup \widetilde{\mathcal{L}}_{B}\left(l_{A}, l_{B} ; u\right) \cup \widetilde{\mathcal{L}}^{\mathcal{I}}\left(l_{A}, l_{B} ; u\right)
$$

and

$$
\begin{aligned}
& \widetilde{\mathcal{L}}_{A}\left(l_{A}, l_{B} ; u\right)=\left\{(h),(a) \in \mathcal{L}\left(l_{A}, l_{B} ; u\right): h_{A}=0 \text { and } a_{A}>l_{A}\right\}, \\
& \widetilde{\mathcal{L}}_{B}\left(l_{A}, l_{B} ; u\right)=\left\{(h),(a) \in \mathcal{L}\left(l_{A}, l_{B} ; u\right): h_{B}=0 \text { and } a_{B}>l_{B}\right\}, \\
& \widetilde{\mathcal{L}}^{\mathcal{I}}\left(l_{A}, l_{B} ; u\right)=\left\{(h),(a) \in \mathcal{L}\left(l_{A}, l_{B} ; u\right): h_{I}=0 \text { and } a_{I}>0\right\} .
\end{aligned}
$$

Assume that $(h),(a) \in \widetilde{\mathcal{L}}\left(l_{A}, l_{B} ; u\right)$, and that $h_{A}>0$ or $h^{\mathcal{I}}>0$. For instance, $(h),(a) \in \widetilde{\mathcal{L}}^{\mathcal{I}}\left(l_{A}, l_{B} ; u\right)$ and $h_{A}>0$. Then, by Lemma B.1(iv), $\widetilde{Q}$ strictly increases when $a_{A}$ is replaced by $a_{A}+a^{\mathcal{I}}$ and $a^{\mathcal{I}}$ by 0 . This contradicts the fact that $(h),(a)$ is a maximizer. Next, if $(h),(a) \in \widetilde{\mathcal{L}}\left(l_{A}, l_{B} ; u\right)$ and $h_{A}=h^{\mathcal{I}}=0$, then $h_{B}=1$, and the first case is $(h),(a) \in \widetilde{\mathcal{L}}_{A}\left(l_{A}, l_{B} ; u\right)$, while the second case is $(h),(a) \in \widetilde{\mathcal{L}}^{\mathcal{I}}\left(l_{A}, l_{B} ; u\right)$. In the second case, as before, we replace $a_{A}$ by $a_{A}+a^{\mathcal{I}}$ and $a^{\mathcal{I}}$ by 0 , which does not change $\widetilde{Q}$ but yields that $a_{A}>l_{A}$ and therefore brings us back to the first case. In this first case, we are left with an expression of the form

$$
Q((h),(a))=g_{l_{B}}\left(a_{B}, 1\right)+a_{B} \frac{\beta-\alpha}{2}
$$

with $h_{A}=h^{\mathcal{I}}=0$ and $a_{A}>l_{A}$. Thus, if we can show that there exists an $x \in(0,1)$ such that

$$
g_{l_{A}}\left(a_{A}, x\right)+g_{l_{B}}\left(a_{B}, 1-x\right)>g_{l_{B}}\left(a_{B}, 1\right),
$$


then we can claim that $(h),(a)$ is not a maximizer of $(\mathrm{C} .18)$ and the proof for $(h),(a) \notin \widetilde{\mathcal{L}}\left(l_{A}, l_{B} ; u\right)$ will be complete.

To that end, we recall (4.4), which allows us to rewrite the left-hand side in (C.23) as

$$
g_{l_{A}}\left(a_{A}, x\right)+g_{l_{B}}\left(a_{B}, 1-x\right)=a_{A} \kappa\left(\frac{a_{A}}{l_{A}}, \frac{x}{l_{A}}\right)+a_{B} \kappa\left(\frac{a_{B}}{l_{B}}, \frac{1-x}{l_{B}}\right)+a_{B} \frac{\beta-\alpha}{2} .
$$

We recall den Hollander and Whittington (2006), Lemma 2.1.1, which claims that $\kappa$ is defined on DOM $=\{(a, b): a \geq 1+b, b \geq 0\}$, is analytic on the interior of DOM and is continuous on DOM. Moreover, in the proof of this lemma, an expression for $\partial_{b} \kappa(a, b)$ is provided, which is valid on the interior of DOM. From this expression we can easily check that if $a>1$, then $\lim _{b \rightarrow 0} \partial_{b} \kappa(a, b)=\infty$. Therefore, by the continuity of $\kappa$ on $\left(a_{A} / l_{A}, 0\right)$ with $a_{A} / l_{A}>1$ we can assert that the derivative with respect to $x$ of the left-hand side in (C.24) at $x=0$ is infinite, and therefore there exists an $x>0$ such that (C.23) is satisfied.

It remains to prove the strict concavity of $u \mapsto u \psi(\Theta, u)$ with $\Theta \in \mathcal{V}_{\text {int }, M}$. Pick $u_{1}>u_{2} \geq t_{\Theta}$, and let $\left(h_{1}\right),\left(a_{1}\right) \in \mathcal{L}\left(l_{A}, l_{B} ; u_{1}\right)$ and $\left(h_{2}\right),\left(a_{2}\right) \in \mathcal{L}\left(l_{A}, l_{B} ; u_{2}\right)$ be maximizers of (C.18) for $u_{1}$ and $u_{2}$, respectively. We can write

$$
\begin{aligned}
& \left(a_{1}\right),\left(h_{1}\right)=\left(a_{A, 1}, a_{B, 1}, a_{1}^{\mathcal{I}}\right),\left(h_{A, 1}, h_{B, 1}, h_{1}^{\mathcal{I}}\right), \\
& \left(a_{2}\right),\left(h_{2}\right)=\left(a_{A, 2}, a_{B, 2}, a_{2}^{\mathcal{I}}\right),\left(h_{A, 2}, h_{B, 2}, h_{2}^{\mathcal{I}}\right) .
\end{aligned}
$$

Thus, $\left(\frac{a_{1}+a_{2}}{2}\right),\left(\frac{h_{1}+h_{2}}{2}\right) \in \mathcal{L}\left(l_{A}, l_{B} ; \frac{u_{1}+u_{2}}{2}\right)$ and, with the help of the concavity of $g_{l_{A}}, g_{l_{B}}, \widetilde{g}$ proven above, we can write

$$
\frac{u_{1}+u_{2}}{2} \psi\left(\Theta, \frac{u_{1}+u_{2}}{2}\right) \geq \widetilde{Q}\left(\left(\frac{a_{1}+a_{2}}{2}\right),\left(\frac{h_{1}+h_{2}}{2}\right)\right) \geq \frac{1}{2}\left(u_{1} \psi\left(\Theta, u_{1}\right)+u_{2} \psi\left(\Theta, u_{2}\right)\right) \text {. }
$$

At this stage, we assume that the right-most inequality in (C.26) is an equality and show that this leads to a contradiction, after which Lemma C.4 will be proven.

We have proven above that $\left(a_{1}\right), \quad\left(h_{1}\right) \notin \widetilde{\mathcal{L}}\left(l_{A}, l_{B} ; u_{1}\right)$ and $\left(a_{2}\right)$, $\left(h_{2}\right) \notin \widetilde{\mathcal{L}}\left(l_{A}, l_{B} ; u_{2}\right)$. Thus, we can use (C.19) and the strict concavity of $g_{l_{A}}, g_{l_{B}}$ on $\mathcal{N}_{l_{A}}^{+}, \mathcal{N}_{l_{B}}^{+}$and the concavity of $\widetilde{g}$ on $\mathcal{N}_{0}$ to conclude that necessarily

$$
\left(a_{A, 1}, h_{A, 1}\right)=\left(a_{A, 2}, h_{A, 2}\right) \quad \text { and } \quad\left(a_{B, 1}, h_{B, 1}\right)=\left(a_{B, 2}, h_{B, 2}\right) .
$$

As a consequence, we recall that $u_{1}>u_{2}$ and we can write

$$
u_{1}^{\mathcal{I}}=u_{1}-a_{A, 1}-a_{B, 2}>u_{2}-a_{A, 2}-a_{B, 2}=u_{2}^{\mathcal{I}} \geq 0,
$$

and therefore, since $\left(a_{1}\right),\left(h_{1}\right) \notin \widetilde{\mathcal{L}}^{\mathcal{I}}\left(l_{A}, l_{B} ; u_{1}\right)$, it follows that $h_{1}^{\mathcal{I}}>0$ such that (recall $(\mathrm{C} .27)$ )

$$
h_{1}^{\mathcal{I}}=1-h_{A, 1}-h_{B, 1}=1-h_{A, 2}-h_{B, 2}=h_{2}^{\mathcal{I}}>0 .
$$

Hence we can use the strict concavity of $a \mapsto \widetilde{g}\left(a, h_{1}^{\mathcal{I}}\right)$ to conclude that $u_{1}^{\mathcal{I}}=u_{2}^{\mathcal{I}}$, which clearly contradicts (C.28).

C.3.3. Proof of Proposition C.5. The proof is performed with the help of Lemma E.1 stated in Section E. For this reason we use some notations introduced in Lemma E.1.

We pick $\gamma, \eta>0$ (which will be specified later), and we let $\widehat{K} \in \mathbb{N}$ be the integer in Lemma E.1 associated with $\alpha, \beta, \eta, \gamma$. For $\Theta \in \overline{\mathcal{V}}_{M}, u \geq t_{\Theta}$ and $\pi \in \mathcal{W}_{\Theta, u, L}$, we let $N_{\pi}$ be the number of excursions of $\pi$ in solvent $B$ in columns of type $\Theta$. We further let also $\left(I_{\pi}\right)=\left(I_{\pi}(1), \ldots, I_{\pi}\left(N_{\pi}\right)\right)$ be the sequence of consecutive intervals in $\{1, \ldots, u L\}$ on which $\pi$ makes these $N_{\pi}$ excursions in $B$, so that $\left(I_{\pi}\right) \in \mathcal{E}_{u L, N_{\pi}}$ and $T(\pi)=\sum_{i=1}^{N_{\pi}}\left|I_{\pi}(i)\right|$. 
Pick $\Theta \in \overline{\mathcal{V}}_{M}, u \geq t_{\Theta}$ and partition $\mathcal{W}_{\Theta, u, L}$ into two parts:

$V_{u, L, \gamma}^{\Theta,+}=\left\{\pi \in \mathcal{W}_{\Theta, u, L}: T(\pi) \geq \gamma u L\right\} \quad$ and $\quad V_{u, L, \gamma}^{\Theta,-}=\left\{\pi \in \mathcal{W}_{\Theta, u, L}: T(\pi) \leq \gamma u L\right\}$.

There exists a $c>0$, depending on $\alpha, \beta$ only, such that

$$
\left|H_{L}^{\Theta, \omega}(\pi)-T(\pi) \frac{\beta-\alpha}{2}\right| \leq c T(\pi) \leq c \gamma u L, \quad \pi \in V_{u, L, \gamma}^{\Theta,-} .
$$

Since any excursion in solvent $B$ requires at least 1 horizontal steps or $L$ vertical steps, we have that $N_{\pi} \leq u+L$ for $\pi \in \mathcal{W}_{\Theta, u, L}$. Since $u+L \leq u L / \widehat{K}$ as soon as $u, L \geq 2 \widehat{K}$, it follows that

$$
I(\pi) \in \cup_{N=1}^{u L / \widehat{K}}\left\{I \in \mathcal{E}_{u L, N}: T(I) \geq \gamma u L\right\}, \quad L \geq 2 \widehat{K}, \quad u \geq t_{\Theta} \vee 2 \widehat{K}, \quad \pi \in V_{u, L, \gamma}^{\Theta,+},
$$

and therefore $\omega \in Q_{u L, \widehat{K}}^{\gamma, \eta}$ implies that $\left|H_{L}^{\Theta, \omega}(\pi)-T(\pi) \frac{\beta-\alpha}{2}\right| \leq \eta u L$ for $\pi \in V_{u, L, \gamma}^{\Theta,+}$. Consequently, for $\omega \in Q_{u L, \widehat{K}}^{\gamma, \eta}$, we have

$\left|H_{L}^{\Theta, \omega}(\pi)-T(\pi) \frac{\beta-\alpha}{2}\right| \leq u L(\eta+c \gamma), \quad \Theta \in \overline{\mathcal{V}}_{M}, \quad u \geq 2 \widehat{K} \vee t_{\Theta}, \quad L \geq 2 \widehat{K}, \quad \pi \in \mathcal{W}_{\Theta, u, L}$

Rewrite

$$
\psi_{L}(\Theta, u)=\mathbb{E}\left[\frac{1}{u L} \log \sum_{\pi \in \mathcal{W}_{\Theta, u, L}} e^{H_{L}^{\Theta, \omega}(\pi)} \mid \mathcal{Q}_{u L, \widehat{K}}^{\gamma, \eta}\right]+\mathbb{P}\left(\left(\mathcal{Q}_{u L, \widehat{K}}^{\gamma, \eta}\right)^{c}\right) \Delta,
$$

where $\Delta$ is an error term given by

$$
\Delta=\mathbb{E}\left[\frac{1}{u L} \log \sum_{\pi \in \mathcal{W}_{\Theta, u, L}} e^{H_{L}^{\Theta, \omega}(\pi)} \mid\left(\mathcal{Q}_{u L, \widehat{K}}^{\gamma, \eta}\right)^{c}\right]-\mathbb{E}\left[\frac{1}{u L} \log \sum_{\pi \in \mathcal{W}_{\Theta, u, L}} e^{H_{L}^{\Theta, \omega}(\pi)} \mid \mathcal{Q}_{u L, \widehat{K}}^{\gamma, \eta}\right] .
$$

By (4.37), we obtain that $|\Delta| \leq 2 C_{\text {uf }}$.

To conclude, we set $\eta=\varepsilon / 3, \gamma=\varepsilon / 3 c$. By Lemma E.1, there exists an $L_{\varepsilon} \in \mathbb{N}$ such that, for $u \geq 2 \widehat{K} \vee t_{\Theta}$ and $L \geq L_{\varepsilon}$, we have $\mathbb{P}\left(\left(\mathcal{Q}_{u L, \widehat{K}}^{\gamma, \eta}\right)^{c}\right) \leq \varepsilon / 6 C_{\text {uf. Thus, we }}$ can use (C.33) and (C.34) to complete the proof of Proposition C.5.

C.3.4. Proof of Lemma C.6. Pick $\varepsilon>0$. By applying Proposition C.5 with $\varepsilon / 2$, we see that there exists an $R_{\varepsilon / 2}>0$ such that

$$
\psi(\Theta, u) \leq \limsup _{L \rightarrow \infty} \frac{1}{u L} \log \sum_{\pi \in \mathcal{W}_{\Theta, u, L}} e^{T(\pi) \frac{\beta-\alpha}{2}}+\frac{\varepsilon}{2}, \quad \Theta \in \overline{\mathcal{V}}_{M}, \quad u \geq t_{\Theta} \vee R_{\varepsilon / 2} .
$$

We first consider the case $\Theta \in \overline{\mathcal{V}}_{M} \backslash \overline{\mathcal{V}}_{\text {nint }, B, 1, M}$. Since $(\alpha, \beta) \in$ CONE, we can use (C.36) to obtain

$$
\psi(\Theta, u) \leq \limsup _{L \rightarrow \infty} \frac{1}{u L} \log \left|\mathcal{W}_{\Theta, u, L}\right|+\frac{\varepsilon}{2}, \quad u \geq t_{\Theta} \vee R_{\varepsilon / 2} .
$$

Thus, (C.37) and Lemma A.2 imply that there exists a $C_{\varepsilon} \geq R_{\varepsilon / 2}$ such that $\psi(\Theta, u) \leq \varepsilon$ when $u \geq t_{\Theta} \vee C_{\varepsilon}$ and $\Theta \in \overline{\mathcal{V}}_{M} \backslash \overline{\mathcal{V}}_{\text {nint }, B, 1, M}$. The case $\Theta \in \overline{\mathcal{V}}_{\text {nint }, B, 1, M}$ can be treated similarly after noticing that $T(\pi)=u L$ for $\pi \in \mathcal{W}_{\Theta, u, L}$ and $\Theta \in \overline{\mathcal{V}}_{\text {nint }, B, 1, M}$.

C.3.5. Proof of Lemma C.7. The proof is a straightforward consequence of the strict concavity of $u \mapsto u \psi(\Theta, u)$ for $\Theta \in \overline{\mathcal{V}}_{M}$, Proposition C.5 and Lemma A.2. 
C.3.6. Proof of Lemma C.8. Pick $\varepsilon>0$. The proof will be complete once we show the following two properties:

(1) There exists a $T_{\varepsilon}>0$ such that

$$
\partial_{u}^{-}(u \psi(\Theta, u))\left(2 t_{\Theta}\right) \leq \begin{cases}\varepsilon & \text { if } \quad \Theta \in \overline{\mathcal{V}}_{M} \backslash \overline{\mathcal{V}}_{\text {nint }, B, 1, M}: t_{\Theta} \geq T_{\varepsilon}, \\ \frac{\beta-\alpha}{2}+\varepsilon & \text { if } \quad \Theta \in \overline{\mathcal{V}}_{\text {nint }, B, 1, M}: t_{\Theta} \geq T_{\varepsilon} .\end{cases}
$$

(2) For all $T>0$ there exists a $V_{\varepsilon, T}>0$ such that

$\partial_{u}^{-}(u \psi(\Theta, u))(v) \leq\left\{\begin{array}{lll}\varepsilon & \text { if } \quad \Theta \in \overline{\mathcal{V}}_{M} \backslash \overline{\mathcal{V}}_{\text {nint }, B, 1, M}: t_{\Theta} \leq T, \quad v \geq t_{\Theta} \vee V_{\varepsilon, T}, \\ \frac{\beta-\alpha}{2}+\varepsilon & \text { if } \quad \Theta \in \overline{\mathcal{V}}_{\text {nint }, B, 1, M}: t_{\Theta} \leq T, \quad v \geq t_{\Theta} \vee V_{\varepsilon, T} .\end{array}\right.$

We prove (C.39) for the case $\Theta \in \mathcal{V}_{M} \backslash \overline{\mathcal{V}}_{\text {nint }, B, 1, M}$ (the case $\Theta \in \mathcal{V}_{\text {nint, } B, 1, M}$ can be treated similarly). To that aim, we assume that there exists a sequence $\left(\Theta_{n}\right)_{n \in \mathbb{N}}$ in $\mathcal{V}_{M} \backslash \overline{\mathcal{V}}_{\text {nint, } B, 1, M}$ such that $t_{\Theta_{n}} \leq T$ for $n \in \mathbb{N}$ and a sequence $\left(u_{n}\right)_{n \in \mathbb{N}}$ such that $u_{n} \geq t_{\Theta_{n}}$ for $n \in \mathbb{N}, \lim _{n \rightarrow \infty} u_{n}=\infty$ and

$$
\partial_{u}^{-}\left(u \psi\left(\Theta_{n}, u\right)\right)\left(u_{n}\right) \geq \varepsilon, \quad n \in \mathbb{N} .
$$

By concavity of $u \mapsto u \psi\left(\Theta_{n}, u\right)$ for $n \in \mathbb{N}$ (see Lemma C.4), we have

$$
u_{n} \psi\left(\Theta_{n}, u_{n}\right)-t_{\Theta_{n}} \psi\left(\Theta_{n}, t_{\Theta_{n}}\right) \geq \varepsilon\left(u_{n}-t_{\Theta_{n}}\right), \quad n \in \mathbb{N} .
$$

Therefore, the uniform bound on free energies in (4.37) and the inequality $t_{\Theta_{n}} \leq T$ allow us to rewrite (C.41) as

$$
\psi\left(\Theta_{n}, u_{n}\right) \geq \varepsilon-\frac{T\left(C_{\mathrm{uf}}+\varepsilon\right)}{u_{n}}, \quad n \in \mathbb{N},
$$

which contradicts Lemma C. 6 because $\lim _{n \rightarrow \infty} u_{n}=\infty$.

It remains to prove (C.38). This is done in a similar manner for the case $\Theta \in \mathcal{V}_{M} \backslash$ $\overline{\mathcal{V}}_{\text {nint }, B, 1, M}$ (the case $\Theta \in \mathcal{V}_{\text {nint }, B, 1, M}$ can again be treated similarly), by assuming that there exists a sequence $\left(\Theta_{n}\right)_{n \in \mathbb{N}}$ in $\mathcal{V}_{M} \backslash \overline{\mathcal{V}}_{\text {nint }, B, 1, M}$ such that $\lim _{n \rightarrow \infty} t_{\Theta_{n}}=\infty$ and

$$
\partial_{u}^{-}\left(u \psi\left(\Theta_{n}, u\right)\right)\left(2 t_{\Theta_{n}}\right) \geq \varepsilon, \quad n \in \mathbb{N} .
$$

Thus, similarly as in (C.41-C.42), the concavity of $u \mapsto u \psi\left(\Theta_{n}, u\right)$ and (C.43) give

$$
\psi\left(\Theta_{n}, 2 t_{\Theta_{n}}\right) \geq \frac{\varepsilon}{2}+\frac{\psi\left(\Theta_{n}, t_{\Theta_{n}}\right)}{2}, \quad n \in \mathbb{N} .
$$

At this point we use Proposition C.5 to assert that there exist $R_{\varepsilon}>0$ and $L_{\varepsilon} \in \mathbb{N}$ such that, for $n$ satisfying $t_{\Theta_{n}} \geq R_{\varepsilon}$ and $L \geq L_{\varepsilon}$, we have

$$
\begin{gathered}
\psi\left(\Theta_{n}, t_{\Theta_{n}}\right) \geq \frac{1}{t_{\Theta_{n}} L} \log \sum_{\pi \in \mathcal{W}_{\Theta, t_{\Theta_{n}}, L}} e^{T(\pi) \frac{\beta-\alpha}{2}}-\frac{\varepsilon}{4}, \\
\psi\left(\Theta_{n}, 2 t_{\Theta_{n}}\right) \leq \frac{1}{2 t_{\Theta_{n} L} L} \log \sum_{\pi \in \mathcal{W}_{\Theta_{n}, 2 t_{\Theta_{n}}, L}} e^{T(\pi) \frac{\beta-\alpha}{2}}+\frac{\varepsilon}{4} .
\end{gathered}
$$

By using (C.44-C.45), we obtain that, for $t_{\Theta_{n}} \geq R_{\varepsilon}$ and $L \geq L_{\varepsilon}$,

$$
\frac{1}{2 t_{\Theta_{n}} L} \log \sum_{\pi \in \mathcal{W}_{\Theta_{n}, 2 t_{\Theta_{n}}, L}} e^{T(\pi) \frac{\beta-\alpha}{2}} \geq \frac{1}{2 t_{\Theta_{n}} L} \log \sum_{\pi \in \mathcal{W}_{\Theta, t_{\Theta_{n}}, L}} e^{T(\pi) \frac{\beta-\alpha}{2}}+\frac{\varepsilon}{8},
$$


uses some key ingredients that are provided which we can rewrite as

$$
\begin{aligned}
\frac{1}{2 t_{\Theta_{n}} L} \log \left|\mathcal{W}_{\Theta_{n}, 2 t_{\Theta_{n}}, L}\right|+\frac{\beta-\alpha}{4 t_{\Theta_{n}} L} & \min \left\{T(\pi), \pi \in \mathcal{W}_{\Theta_{n}, 2 t_{\Theta_{n}}, L}\right\} \\
& \geq \frac{\beta-\alpha}{4 t_{\Theta_{n}} L} \min \left\{T(\pi), \pi \in \mathcal{W}_{\Theta_{n}, t_{\Theta_{n}}, L}\right\}+\frac{\varepsilon}{8} .
\end{aligned}
$$

Since $\Theta_{n} \in \mathcal{V}_{M} \backslash \overline{\mathcal{V}}_{\text {nint }, B, 1, M}$, there exist $\pi_{1} \in \mathcal{W}_{\Theta_{n}, t_{\Theta_{n}}, L}$ and $\pi_{2} \in \mathcal{W}_{\Theta_{n}, 2 t_{\Theta_{n}}, L}$ such that

$$
\begin{aligned}
& T\left(\pi_{1}\right)=l_{B}\left(\Theta_{n}\right)=\min \left\{T(\pi), \pi \in \mathcal{W}_{\Theta_{n}, t_{\Theta_{n}}, L}\right\}, \\
& T\left(\pi_{2}\right)=l_{B}\left(\Theta_{n}\right)=\min \left\{T(\pi), \pi \in \mathcal{W}_{\Theta_{n}, 2 t_{\Theta_{n}}, L}\right\} .
\end{aligned}
$$

Thus, for $t_{\Theta_{n}} \geq R_{\varepsilon}$ and $L \geq L_{\varepsilon}$, the inequality in (C.47) becomes

$$
\frac{1}{2 t_{\Theta_{n}} L} \log \left|\mathcal{W}_{\Theta_{n}, 2 t_{\Theta_{n}}, L}\right| \geq \frac{\varepsilon}{8}
$$

which obviously contradicts Lemma A.2.

\section{Appendix D. Concentration of measure}

Let $\mathcal{S}$ be a finite set and let $\left(X_{i}, \mathcal{A}_{i}, \mu_{i}\right)_{i \in \mathcal{S}}$ be a family of probability spaces. Consider the product space $X=\prod_{i \in \mathcal{S}} X_{i}$ endowed with the product $\sigma$-field $\mathcal{A}=$ $\otimes_{i \in \mathcal{S}} \mathcal{A}_{i}$ and with the product probability measure $\mu=\otimes_{i \in \mathcal{S}} \mu_{i}$.

Theorem D.1. (Talagrand, 1996) Let $f: X \mapsto \mathbb{R}$ be integrable with respect to $(\mathcal{A}, \mu)$ and, for $i \in \mathcal{S}$, let $d_{i}>0$ be such that $|f(x)-f(y)| \leq d_{i}$ when $x, y \in X$ differ in the $i$-th coordinate only. Let $D=\sum_{i \in \mathcal{S}} d_{i}^{2}$. Then, for all $\varepsilon>0$,

$$
\mu\left\{x \in X:\left|f(x)-\int f d \mu\right|>\varepsilon\right\} \leq 2 e^{-\frac{\varepsilon^{2}}{2 D}} .
$$

The following corollary of Theorem D.1 was used several times in the paper. Let $(\alpha, \beta) \in \mathrm{CONE}$ and let $\left(\xi_{i}\right)_{i \in \mathbb{N}}$ be an i.i.d. sequence of Bernouilli trials taking the values $-\alpha$ and $\beta$ with probability $\frac{1}{2}$ each. Let $l \in \mathbb{N}, T:\left\{(x, y) \in \mathbb{Z}^{2} \times \mathbb{Z}^{2}:|x-y|=\right.$ $1\} \rightarrow\{0,1\}$ and $\Gamma \subset \mathcal{W}_{l}$ (recall $\left.(2.1)\right)$. Let $F_{l}:[-\alpha, \alpha]^{l} \rightarrow \mathbb{R}$ be such that

$$
F_{l}\left(x_{1}, \ldots, x_{l}\right)=\log \sum_{\pi \in \Gamma} e^{\sum_{i=1}^{l} x_{i} T\left(\left(\pi_{i-1}, \pi_{i}\right)\right)} .
$$

For all $x, y \in[-\alpha, \alpha]^{l}$ that differ in one coordinate only we have $\left|F_{l}(x)-F_{l}(y)\right| \leq$ $2 \alpha$. Therefore we can use Theorem D.1 with $\mathcal{S}=\{1, \ldots, l\}, X_{i}=[-\alpha, \alpha]$ and $\mu_{i}=\frac{1}{2}\left(\delta_{-\alpha}+\delta_{\beta}\right)$ for all $i \in \mathcal{S}$, and $D=4 \alpha^{2} l$, to obtain that there exist $C_{1}, C_{2}>0$ such that, for every $l \in \mathbb{N}, \Gamma \subset \mathcal{W}_{n}$ and $T:\left\{(x, y) \in \mathbb{Z}^{2} \times \mathbb{Z}^{2}:|x-y|=1\right\} \rightarrow\{0,1\}$,

$$
\mathbb{P}\left(\left|F_{l}\left(\xi_{1}, \ldots, \xi_{m}\right)-\mathbb{E}\left(F_{l}\left(\xi_{1}, \ldots, \xi_{m}\right)\right)\right|>\eta\right) \leq C_{1} e^{-\frac{C_{2} \eta^{2}}{l}}
$$

\section{Appendix E. Large deviation estimate}

Let $\left(\xi_{i}\right)_{i \in \mathbb{N}}$ be an i.i.d. sequence of Bernouilli trials taking values $\beta$ and $-\alpha$ with probability $\frac{1}{2}$ each. For $N \leq n \in \mathbb{N}$, denote by $\mathcal{E}_{n, N}$ the set of all ordered sequences of $N$ disjoint and non-empty intervals included in $\{1, \ldots, n\}$, i.e.,

$$
\begin{gathered}
\mathcal{E}_{n, N}=\left\{\left(I_{j}\right)_{1 \leq j \leq N} \subset\{1, \ldots, n\}: I_{j}=\left\{\min I_{j}, \ldots, \max I_{j}\right\} \forall 1 \leq j \leq N,\right. \\
\left.\max I_{j}<\min I_{j+1} \forall 1 \leq j \leq N-1 \text { and } I_{j} \neq \emptyset \forall 1 \leq j \leq N\right\} .
\end{gathered}
$$


For $(I) \in \mathcal{E}_{n, N}$, let $T(I)=\sum_{j=1}^{N}\left|I_{j}\right|$ be the cumulative length of the intervals making up $(I)$. Pick $\gamma>0$ and $K \in \mathbb{N}$, and denote by $\widehat{\mathcal{E}}_{n, K}^{\gamma}$ the set of those $(I)$ in $\cup_{1 \leq N \leq(n / K)} \mathcal{E}_{n, N}$ that have a cumulative length larger than $\gamma n$, i.e.,

$$
\widehat{\mathcal{E}}_{n, K}^{\gamma}=\cup_{N=1}^{n / K}\left\{(I) \in \mathcal{E}_{n, N}: T(I) \geq \gamma n\right\} .
$$

Next, for $\eta>0$ set

$$
\mathcal{Q}_{n, K}^{\gamma, \eta}=\bigcap_{(I) \in \widehat{\mathcal{E}}_{n, K}^{\gamma}}\left\{\left|\sum_{j=1}^{N} \sum_{i \in I_{j}}\left(\xi_{i}-\frac{\beta-\alpha}{2}\right)\right| \leq \eta T(I)\right\} .
$$

Lemma E.1. For all $(\alpha, \beta) \in \mathrm{CONE}, \gamma>0$ and $\eta>0$ there exists an $\widehat{K} \in \mathbb{N}$ such that, for all $K \geq \widehat{K}$,

$$
\lim _{n \rightarrow \infty} P\left(\left(\mathcal{Q}_{n, K}^{\gamma, \eta}\right)^{c}\right)=0 .
$$

Proof: An application of Cramér's theorem for i.i.d. random variables gives that there exists a $c_{\eta}>0$ such that, for every $(I) \in \widehat{\mathcal{E}}_{n, K}^{\gamma}$,

$$
\mathbb{P}_{\xi}\left(\left|\sum_{j=1}^{N} \sum_{i \in I_{j}}\left(\xi_{i}-\frac{\beta-\alpha}{2}\right)\right| \geq \eta T(I)\right) \leq e^{-c_{\eta} T(I)} \leq e^{-c_{\eta} \gamma n},
$$

where we use that $T(I) \geq \gamma n$ for every $(I) \in \widehat{\mathcal{E}}_{n, K}^{\gamma}$. Therefore

$$
\mathbb{P}_{\xi}\left(\left(\mathcal{Q}_{n, K}^{\gamma, \eta}\right)^{c}\right) \leq\left|\widehat{\mathcal{E}}_{n, K}^{\gamma}\right| e^{-c(\eta) \gamma n}
$$

and it remains to bound $\left|\widehat{\mathcal{E}}_{n, K}^{\gamma}\right|$ as

$$
\widehat{\mathcal{E}}_{n, K}^{\gamma}=\sum_{N=1}^{n / K}\left|\left\{(I) \in \mathcal{E}_{n, N}: T(I) \geq \gamma n\right\}\right| \leq \sum_{N=1}^{n / K}\left(\begin{array}{c}
n \\
2 N
\end{array}\right),
$$

where we use that choosing $(I) \in \mathcal{E}_{n, N}$ amounts to choosing in $\{1, \ldots, n\}$ the end points of the $N$ disjoint intervals. Thus, the right-hand side of (E.7) is at most $(n / K)\left(\begin{array}{c}n \\ 2 n / K\end{array}\right)$, which for $K$ large enough is $o\left(e^{c(\eta) \gamma n}\right)$ as $n \rightarrow \infty$.

\section{Appendix F. On the maximizers of the slope-based variational formula}

In this appendix we prove that, when restricted to $\overline{\mathcal{R}}_{p, M}$, the supremum of the variational formula in (2.14), which equals the truncated free energy $f(\alpha, \beta ; M, p)$, is attained at some $\bar{\rho} \in \overline{\mathcal{R}}_{p, M}$ and for a unique $\bar{v} \in \overline{\mathcal{B}}$. For ease of notation we suppress the $M, p$-dependence of $f(\alpha, \beta ; M, p)$ in the proofs of this section.

Recall (7.20) and for $M \in \mathbb{N}, p \in(0,1)$ and $(\alpha, \beta) \in \mathrm{CONE}$, let $\mathcal{O}_{p, M, \alpha, \beta}$ be the subset of $\overline{\mathcal{R}}_{p, M}$ containing those $\bar{\rho}$ that maximize the variational formula in (2.14), i.e.,

$$
f(\alpha, \beta ; M, p)=h(\bar{\rho} ; \alpha, \beta)=\sup _{v \in \overline{\mathcal{B}}} \frac{\bar{N}(\bar{\rho}, v)}{\bar{D}(\bar{\rho}, v)} \quad \text { for } \bar{\rho} \in \mathcal{O}_{p, M, \alpha, \beta}
$$

Recall (3.8-3.10) and set

$$
\bar{v}=v(f(\alpha, \beta ; M, p))
$$


Theorem F.1. For all $M \in \mathbb{N}, p \in(0,1)$ and $(\alpha, \beta) \in$ CONE the following hold:

(1) The set $\mathcal{O}_{p, M, \alpha, \beta}$ is non-empty.

(2) For all $\bar{\rho} \in \mathcal{O}_{p, M, \alpha, \beta}$ and all $v \in \overline{\mathcal{B}}$ satisfying $f(\alpha, \beta ; M, p)=\bar{N}(\bar{\rho}, v) / \bar{D}(\bar{\rho}, v)$, $v=\bar{v}$ for $\bar{\rho}$-a.e. $(k, l) \in\{A, B\} \times[0, \infty)$ or $k=\mathcal{I}$.

Proof: The following proposition will be proven in Section F.1 below and tells us that the maximum of the old variational formula in (3.15) is attained for some $\rho \in \mathcal{R}_{p, M}$. Recall the definition of $g(\rho ; \alpha, \beta)$ for $\rho \in \mathcal{R}_{p, M}$ in (7.10).

Theorem F.2. For all $(\alpha, \beta) \in \mathrm{CONE}$, there exists a $\rho \in \mathcal{R}_{p, M}$ such that $f(\alpha, \beta ; M, p)=g(\rho ; \alpha, \beta)$.

We give the proof of Theorem F.1 subject to Theorem F.2. To that aim, we pick $(\alpha, \beta) \in$ CONE and note that, by Theorem F.2, there exists a $\hat{\rho} \in \mathcal{R}_{p, M}$ such that $f(\alpha, \beta)=g(\hat{\rho} ; \alpha, \beta)$. In what follows, we suppress the $(\alpha, \beta)$-dependence of $g(\hat{\rho} ; \alpha, \beta)$.

Since $f(\alpha, \beta)=g(\hat{\rho}),(4.64)$ ensures that $g(\hat{\rho})>0$, and by applying Lemma 7.1 we obtain that

$$
f(\alpha, \beta)=\frac{N(\hat{\rho}, u(f(\alpha, \beta)))}{D(\hat{\rho}, u(f(\alpha, \beta)))} .
$$

Apply Lemma 7.5, which ensures that there exist a $\bar{\rho} \in \overline{\mathcal{R}}_{p}$ and a $v \in \overline{\mathcal{F}}$ such that

$$
\frac{N(\hat{\rho}, u(f(\alpha, \beta)))}{D(\hat{\rho}, u(f(\alpha, \beta)))} \leq \frac{\bar{N}(\bar{\rho}, v)}{\bar{D}(\bar{\rho}, v)} .
$$

Then $h(\bar{\rho})>0$, and we use Lemma 7.3, which tells us that

$$
\frac{\bar{N}(\bar{\rho}, v)}{\bar{D}(\bar{\rho}, v)} \leq \frac{\bar{N}(\bar{\rho}, v(h(\bar{\rho})))}{\bar{D}(\bar{\rho}, v(h(\bar{\rho})))} .
$$

Now (F.3-F.5) and the variational formula in (2.14) are sufficient to complete the proof of (1). The proof of (2) is a straightforward consequence of Lemma 7.1.

F.1. Proof of Theorem F.2. We give the proof of Theorem F.2 subject to the following lemma, which will be proven in Section F.2 below.

Lemma F.3. For all $t>0$ and $u \in \mathcal{B}_{\overline{\mathcal{V}}_{M}}$ there exists an $m_{0} \in \mathbb{N}$ such that, for all $\rho \in \mathcal{R}_{p, M}$ and $v \in \mathcal{B}_{\overline{\mathcal{V}}_{M}}$ satisfying $v \leq u$ and $N(\rho, v) / D(\rho, v) \geq t$, there exists a $\tilde{\rho} \in \mathcal{R}_{p, M}^{m_{0}}$ such that $N(\tilde{\rho}, v) / D(\tilde{\rho}, v) \geq N(\rho, v) / D(\rho, v)$.

Let $\left(\rho_{n}\right)_{n \in \mathbb{N}}$ in $\mathcal{R}_{p, M}$ be such that $n \mapsto g\left(\rho_{n} ; \alpha, \beta\right)$ is increasing with $\lim _{n \rightarrow \infty} g\left(\rho_{n} ; \alpha, \beta\right)=f(\alpha, \beta)$. Obviously we can choose $\left(\rho_{n}\right)_{n \in \mathbb{N}}$ such that $g\left(\rho_{n} ; \alpha, \beta\right) \geq f(\alpha, \beta) / 2$ for all $n \in \mathbb{N}$. Thus, with the help of Lemma 7.1, we obtain

$$
g\left(\rho_{n} ; \alpha, \beta\right)=\frac{N\left(\rho_{n}, u\left(g\left(\rho_{n}\right)\right)\right)}{D\left(\rho_{n}, u\left(g\left(\rho_{n}\right)\right)\right)}, \quad n \in \mathbb{N} .
$$

Apply Lemma F.3 to see that there exists an $m_{0} \in \mathbb{N}$ such that for all $n \in \mathbb{N}$ there exists an $\hat{\rho}_{n} \in \mathcal{R}_{p, M}^{m_{0}}$ such that

$$
\frac{N\left(\hat{\rho}_{n}, u\left(g\left(\rho_{n}\right)\right)\right)}{D\left(\hat{\rho}_{n}, u\left(g\left(\rho_{n}\right)\right)\right)} \geq \frac{N\left(\rho_{n}, u\left(g\left(\rho_{n}\right)\right)\right)}{D\left(\rho_{n}, u\left(g\left(\rho_{n}\right)\right)\right)} .
$$

A straightforward consequence of (F.7) is that

$$
\lim _{n \rightarrow \infty} \frac{N\left(\hat{\rho}_{n}, u\left(g\left(\rho_{n}\right)\right)\right)}{D\left(\hat{\rho}_{n}, u\left(g\left(\rho_{n}\right)\right)\right)}=f(\alpha, \beta) .
$$


Moreover, $\hat{\rho}_{n} \in \mathcal{M}_{1}\left(\overline{\mathcal{V}}_{M}^{m_{0}}\right)$ for all $n \geq n_{0}$, and since $\overline{\mathcal{V}}_{M}^{m_{0}}$ is compact we have that $\hat{\rho}_{n}$ converges weakly to $\rho_{\infty} \in \mathcal{R}_{p, M}^{m_{0}}$ along a subsequence. Lemma B.4 implies that $n \mapsto u\left(g\left(\rho_{n}\right)\right)$ is non-increasing and converges pointwise to $u(f(\alpha, \beta))$ as $n \rightarrow \infty$. Since $\overline{\mathcal{V}}_{M}^{m_{0}}$ is compact, Dini's Theorem tells us that the convergence of $u\left(g\left(\rho_{n}\right)\right)$ to $u(f(\alpha, \beta))$ is uniform on $\overline{\mathcal{V}}_{M}^{m_{0}}$. Therefore, using the uniform continuity of $(u, \Theta) \mapsto$ $u \psi(\Theta, u)$ (see Lemma C.3), we obtain

$$
f(\alpha, \beta)=\frac{N\left(\rho_{\infty}, u(f(\alpha, \beta))\right)}{D\left(\rho_{\infty}, u(f(\alpha, \beta))\right)},
$$

which completes the proof of Theorem F.2.

F.2. Proof of Lemma F.3. First, we state and prove Claim F.4 below, which will be needed to prove Lemma F.3. Pick $m \geq M+2$, and note that for $\Theta=$ $\left(\chi, \Delta \Pi, b_{0}, b_{1}, x\right) \in \overline{\mathcal{V}}_{M} \backslash \overline{\mathcal{V}}_{M}^{m}$ we necessarily have $x_{\Theta}=2$. Define $T_{m}: \overline{\mathcal{V}}_{M} \mapsto \overline{\mathcal{V}}_{M}^{m}$ as

$$
T_{m}(\Theta)= \begin{cases}\Theta & \text { if } \Theta \in \overline{\mathcal{V}}_{M}^{m}, \\ \widetilde{\Theta}=\left(\chi, \Delta \Pi, b_{0}, b_{1}, 1\right) & \text { if } \Theta=\left(\chi, \Delta \Pi, b_{0}, b_{1}, 2\right) \in \overline{\mathcal{V}}_{M} \backslash \overline{\mathcal{V}}_{M}^{m},\end{cases}
$$

Claim F.4. For all $\rho \in \mathcal{R}_{p, M}$ and $m \in \mathbb{N}: m \geq M+2, \rho \circ T_{m}^{-1} \in \mathcal{R}_{p, M}^{m}$.

Proof: First note that $T_{m}: \overline{\mathcal{V}}_{M} \mapsto \overline{\mathcal{V}}_{M}^{m}$ is continuous with respect to the $d_{M^{-}}$ distance. Next, pick $\rho \in \mathcal{R}_{p, M}$. By the definition of $\mathcal{R}_{p, M}$, there exists a strictly increasing sequence $\left(N_{k}\right)_{k \in \mathbb{N}}$ and $\left(\Pi_{j}^{k}\right)_{j \in \mathbb{N}_{0}}, \quad\left(b_{j}^{k}\right)_{j \in \mathbb{N}_{0}}, \quad\left(x_{j}^{k}\right)_{j \in \mathbb{N}_{0}}$ such that $\rho=\lim _{k \rightarrow \infty} \rho_{N_{k}}\left(\Omega, \Pi^{k}, b^{k}, x^{k}\right)$. The continuity of $T_{m}$ implies that

$$
\rho \circ T_{m}^{-1}=\lim _{k \rightarrow \infty} \rho_{N_{k}}\left(\Omega, \Pi^{k}, b^{k}, x^{k}\right) \circ T_{m}^{-1},
$$

and we can easily check that

$$
\rho_{N_{k}}\left(\Omega, \Pi^{k}, b^{k}, x^{k}\right) \circ T_{m}^{-1}=\rho_{N_{k}}\left(\Omega, \Pi^{k}, b^{k}, \tilde{x}^{k}\right),
$$

where for $j, k \in \mathbb{N}_{0}$ we define

$$
\tilde{x}_{j}^{k}= \begin{cases}x_{j}^{k} & \text { if }\left(\Omega(j, \cdot), \Delta \Pi_{j}^{k}, b_{j}^{k}, b_{j+1}^{k} \tilde{x}_{j}^{k}\right) \in \overline{\mathcal{V}}_{M}^{m}, \\ 1 & \text { otherwise. }\end{cases}
$$

Consequently, $\rho \circ T_{m}^{-1} \in \mathcal{R}_{p, M}$.

We resume the proof of Lemma F.3. Pick $t>0, \rho \in \mathcal{R}_{p, M}, u \in \mathcal{B}_{\overline{\mathcal{V}}_{M}}$ and $v \in \mathcal{B}_{\overline{\mathcal{V}}_{M}}$ satisfying $v \leq u$ and $N(\rho, v) / D(\rho, v) \geq t$. Pick $m \in \mathbb{N}: m \geq M+2$, whose value will be specified later, and set $\rho_{m}=\rho \circ T_{m}^{-1}$, which belongs to $\mathcal{R}_{p, M}$ by Claim F.4. Write

$$
\frac{N\left(\rho_{m}, v\right)}{D\left(\rho_{m}, v\right)}-\frac{N(\rho, v)}{D(\rho, v)}=\int_{0}^{1} G^{\prime}(t) d t \quad \text { with } \quad G(t)=\frac{A+t B}{c+t D}
$$

with

$$
\begin{array}{ll}
A=\int_{\overline{\mathcal{V}}_{M}} v_{\Theta} \psi\left(\Theta, v_{\Theta}\right) \rho(d \Theta) & B=\int_{\overline{\mathcal{V}}_{M} \backslash \overline{\mathcal{V}}_{M}^{m}} v_{\widetilde{\Theta}} \psi\left(\widetilde{\Theta}, v_{\widetilde{\Theta}}\right)-v_{\Theta} \psi\left(\Theta, v_{\Theta}\right) \rho(d \Theta) \\
C=\int_{\overline{\mathcal{V}}_{M}} v_{\Theta} \rho(d \Theta) & D=\int_{\overline{\mathcal{V}}_{M} \backslash \overline{\mathcal{V}}_{M}^{m}} v_{\widetilde{\Theta}}-v_{\Theta} \rho(d \Theta) .
\end{array}
$$


Note that the sign of the derivative $G^{\prime}(t)$ is constant and equal to the sign of

$$
B-\frac{A}{C} D=\int_{\overline{\mathcal{V}}_{M} \backslash \overline{\mathcal{V}}_{M}^{m}} v_{\Theta}\left[\frac{A}{C}\left(1-\frac{v_{\widetilde{\Theta}}}{v_{\Theta}}\right)-\psi\left(\Theta, v_{\Theta}\right)+\frac{v_{\widetilde{\Theta}}}{v_{\Theta}} \psi\left(\widetilde{\Theta}, v_{\widetilde{\Theta}}\right)\right] \rho(d \Theta) .
$$

Therefore Lemma F.3 will be proven once we check that for $m$ large enough the right-hand side of (F.17) is strictly positive, uniformly in $v \leq u$. To that aim, we recall Lemma C.6, which tells us that $\psi\left(\Theta, v_{\Theta}\right) \leq t / 2$ for every $\Theta \in \overline{\mathcal{V}}_{M} \backslash \overline{\mathcal{V}}_{M}^{m}$, provided $m$ is chosen large enough (because $v_{\Theta} \geq t_{\Theta} \geq m$ ), and we recall (4.37), which tells us that $\psi\left(\widetilde{\Theta}, v_{\widetilde{\Theta}}\right) \leq C_{\text {uf }}(\alpha)$ for $\Theta \in \overline{\mathcal{V}}_{M} \backslash \overline{\mathcal{V}}_{M}^{m}$. We further note that

$$
v_{\widetilde{\Theta}} \leq \max \left\{u_{\Theta}: \Theta \in \overline{\mathcal{V}}_{M}^{M+2}\right\}<\infty \text { for every } \Theta \in \overline{\mathcal{V}}_{M},
$$

which, together with the fact that $\frac{A}{C}=N(\rho, v) / D(\rho, v) \geq t>0$ and $v_{\Theta} \geq t_{\Theta} \geq m$ for $\Theta \in \overline{\mathcal{V}}_{M} \backslash \overline{\mathcal{V}}_{M}^{m}$, ensures that for $m$ large enough the right-hand side of (F.17) is strictly positive, uniformly in $v \leq u$. This completes the proof of Lemma F.3.

\section{Appendix G. Uniqueness of the maximizers of the variational formula}

In this appendix we first prove, with the help of Lemma G.2, that for $\Theta \in \overline{\mathcal{V}}_{M}$ and $u \geq t_{\Theta}$ the variational formula in Proposition 4.6 has unique maximizers. This uniqueness implies that, for a given column type and a given time spent in the column, the copolymer has a unique way to move through the column. We next use this uniqueness to show, with the help of Proposition G.2, that for $u \in \mathcal{B}_{\overline{\mathcal{V}}_{M}}$ the maximizers of (4.48) are Borel functions of $\Theta \in \overline{\mathcal{V}}_{M}$.

Recall (4.60) and pick $h \in \mathcal{E}$. Set

$$
\begin{aligned}
\mathcal{U}(h)=\left\{\left(r_{A, \Theta}, r_{B, \Theta}, r_{\mathcal{I}, \Theta}\right)_{\Theta \in \overline{\mathcal{V}}_{M}}\right. & \in\left([0, \infty)^{3}\right)^{\overline{\mathcal{V}}_{M}}: \\
& r_{k, \Theta} \geq 1+\frac{l_{k, \Theta}}{h_{k, \Theta}} \forall k \in\{A, B\} \forall \Theta \in \overline{\mathcal{V}}_{M}, \\
& r_{\mathcal{I}, \Theta} \geq 1 \forall k \in\{A, B\} \forall \Theta \in \overline{\mathcal{V}}_{M}, \\
& \left.\Theta \mapsto r_{k, \Theta} \text { Borel } \forall k \in\{A, B, \mathcal{I}\}\right\},
\end{aligned}
$$

where we recall that $\frac{l_{k, \Theta}}{h_{k, \Theta}}=0$ by convention when $l_{k, \Theta}=h_{k, \Theta}=0$.

Proposition G.1. For all $u \in \mathcal{B}_{\overline{\mathcal{V}}_{M}}$ there exist $h \in \mathcal{E}$ and $r \in \mathcal{U}(h)$ such that, for all $\Theta \in \overline{\mathcal{V}}_{M}$,

$$
\begin{aligned}
u_{\Theta} \psi\left(\Theta, u_{\Theta}\right)=h_{A, \Theta} & r_{A, \Theta} \tilde{\kappa}\left(r_{A, \Theta}, \frac{l_{A, \Theta}}{h_{A, \Theta}}\right) \\
& +h_{B, \Theta} r_{B, \Theta}\left[\tilde{\kappa}\left(r_{B, \Theta}, \frac{l_{B, \Theta}}{h_{B, \Theta}}\right)+\frac{\beta-\alpha}{2}\right]+h_{\mathcal{I}, \Theta} r_{\mathcal{I}, \Theta} \phi_{\mathcal{I}}\left(r_{\mathcal{I}, \Theta}\right),
\end{aligned}
$$

and

$$
h_{A, \Theta} r_{A, \Theta}+h_{B, \Theta} r_{B, \Theta}+h_{\mathcal{I}, \Theta} r_{\mathcal{I}, \Theta}=u_{\Theta} .
$$

Proof: For $l \in \mathbb{R}$, let

$$
\mathcal{N}_{l}=\{(a, h) \in[0, \infty) \times[0,1]: a \geq h+|l|\}, \quad \mathcal{N}_{l}^{+}=\left\{(a, h) \in \mathcal{N}_{l}: h>0\right\},
$$

let $g_{l}: \mathcal{N}_{l} \mapsto[0, \infty)$ be defined as $g_{l}(a, h)=a \tilde{\kappa}\left(\frac{a}{h}, \frac{l}{h}\right)$ for $h>0$ and $g_{l}(a, h)=0$ for $h=0$, and let $\tilde{g}: \mathcal{N}_{0} \mapsto[0, \infty)$ be defined as $\tilde{g}(a, h)=a \phi_{\mathcal{I}}\left(\frac{a}{h}\right)$ for $h>0$ and $\tilde{g}(a, h)=0$ for $h=0$. We can rewrite (4.48) as

$$
u \psi(\Theta, u ; \alpha, \beta)=\sup _{(h),(a) \in \mathcal{L}(\Theta ; u)} f_{l_{A}, l_{B}}[(h),(a)]
$$


with

$$
f_{l_{A}, l_{B}}[(h),(a)]=g_{l_{A}}\left(a_{A}, h_{A}\right)+g_{l_{B}}\left(a_{B}, h_{B}\right)+a_{B} \frac{\beta-\alpha}{2}+\tilde{g}\left(a_{\mathcal{I}}, h_{\mathcal{I}}\right) .
$$

Lemma G.2 shows that, subject to some additional conditions, the maximizer in the right-hand side of $(\mathrm{G.5})$ is unique. This allows us to prove the continuity of this maximizer as a function of $\Theta$ on each subset of a finite partition of $\overline{\mathcal{V}}_{M}$, which implies the Borel measurability of this maximizer and completes the proof of Proposition G.1.

Lemma G.2. For all $\Theta \in \overline{\mathcal{V}}_{M}$ and $u \geq t_{\Theta}$ there exists a unique $(\bar{h}),(\bar{a}) \in \mathcal{L}(\Theta ; u)$ satisfying:

(i) $u \psi(\Theta, u ; \alpha, \beta)=f_{l_{A}, l_{B}}[(\bar{h}),(\bar{a})]$.

(ii) $\bar{h}_{k}>0$ if $\bar{a}_{k}>0$ for $k \in\{A, B, \mathcal{I}\}$.

(iii) $\bar{a}_{k}=\bar{h}_{k}=0$ if $\bar{l}_{k}=0$ for $\Theta \in \overline{\mathcal{V}}_{\text {int }, M}$ and $k \in\{A, B\}$.

(iv) $\bar{a}_{k}=\bar{h}_{k}=0$ if $\bar{l}_{k}=0$ for $\Theta \in \overline{\mathcal{V}}_{\text {nint }, k, 2, M}$ and $k \in\{A, B\}$.

Proof: We prove existence and uniqueness.

Existence. The existence of a $\left(h_{1}\right),\left(a_{1}\right) \in \mathcal{L}(\Theta ; u)$ satisfying (i) is ensured by the continuity of $f_{l_{A}, l_{B}}$ and the compactness of $\mathcal{L}(\Theta ; u)$. Assume that $\Theta \in \overline{\mathcal{V}}_{\text {int }, M}$, $l_{A}=0$ and $\left(h_{1, A}, a_{1, A}\right) \neq(0,0)$. Then

$$
\begin{aligned}
g_{0}\left(a_{1, A}, h_{1, A}\right)+\tilde{g}\left(a_{1, \mathcal{I}}, h_{1, \mathcal{I}}\right) & \leq \tilde{g}\left(a_{1, A}, h_{1, A}\right)+\tilde{g}\left(a_{1, \mathcal{I}}, h_{1, \mathcal{I}}\right) \\
& \leq 2 \tilde{g}\left(\frac{a_{1, A}+a_{1, \mathcal{I}}}{2}, \frac{h_{1, A}+h_{1, \mathcal{I}}}{2}\right) \\
& =\tilde{g}\left(a_{1, A}+a_{1, \mathcal{I}}, h_{1, A}+h_{1, \mathcal{I}}\right),
\end{aligned}
$$

where we use the inequality $g_{0} \leq \tilde{g}$ and the concavity of $\tilde{g}$. Thus, by setting $\left(h_{2}\right),\left(a_{2}\right)=\left(0, h_{1, B}, h_{1, A}+h_{1, \mathcal{I}}\right),\left(0, a_{1, B}, a_{1, A}+a_{1, \mathcal{I}}\right)$, we obtain that $\left(h_{2}\right),\left(a_{2}\right) \in$ $\mathcal{L}(\Theta ; u)$, satisfies (iii) and

$$
f_{l_{A}, l_{B}}\left(\left(h_{2}\right),\left(a_{2}\right)\right) \geq f_{l_{A}, l_{B}}\left(\left(h_{1}\right),\left(a_{1}\right)\right),
$$

which implies that $\left(h_{2}\right),\left(a_{2}\right)$ also satisfies (i). The case $\Theta \in \overline{\mathcal{V}}_{\text {int }, M}, l_{B}=0$ and the case $\Theta \in \overline{\mathcal{V}}_{\text {nint }, k, 2, M}, l_{k}=0, k \in\{A, B\}$, can be treated similarly, to conclude that there exist $(h),(a) \in \mathcal{L}(\Theta ; u)$ satisfying (i), (iii-iv). We will show that (ii) follows from these as well. The proof will be given for the case $\Theta \in \overline{\mathcal{V}}_{\text {int, } M}$ and $l_{A}, l_{B}>0$, since (iii) already indicates that $h_{k}=a_{k}=0$ if $l_{k}=0$ for $k \in\{A, B\}$ and $\Theta \in \overline{\mathcal{V}}_{\text {int }, M}$. The case $\Theta \in \overline{\mathcal{V}}_{\text {nint }, M}$ can be treated similarly.

In the proof of Lemma C.4 we showed that $(h),(a) \in \mathcal{L}(\Theta, u)$ maximizing $(\mathrm{G} .5)$ necessarily satisfies $h_{k}>0$ if $a_{k}>l_{k}$ for $k \in\{A, B\}$ and $h_{\mathcal{I}}>0$ if $a_{\mathcal{I}}>0$. Thus, we only need to exclude the cases $h_{k}=0$ and $a_{k}=l_{k}>0$ for $k \in\{A, B\}$. We will therefore assume that $h_{B}=0$ and $a_{B}=l_{B}$, and prove that this leads to a contradiction. The case $h_{A}=0$ and $a_{A}=l_{A}$ is easier to deal with. We finally assume that $a_{\mathcal{I}}>h_{\mathcal{I}}>0$ (the case $a_{\mathcal{I}}=h_{\mathcal{I}}$ being easier). We pick $c>1$ and $x>0$ small enough to ensure that $a_{\mathcal{I}}-c x>h_{\mathcal{I}}-x>0$, and we set $(h)_{x},(a)_{x}=$ $\left(h_{A}, x, h_{\mathcal{I}}-x\right),\left(a_{A}, l_{B}+c x, a_{I}-c x\right)$. The proof will be complete once we show that for $x$ small enough the quantity

$$
f_{l_{A}, l_{B}}\left((h)_{x},(a)_{x}\right)-f_{l_{A}, l_{B}}((h),(a))=g_{l_{B}}\left(l_{B}+c x, x\right)-V_{x}+c x\left(\frac{\beta-\alpha}{2}\right)
$$

is strictly positive with $V_{x}=\tilde{g}\left(a_{\mathcal{I}}, h_{\mathcal{I}}\right)-\tilde{g}\left(a_{\mathcal{I}}-c x, h_{\mathcal{I}}-x\right)$.

At this stage, we note that $\mu \mapsto \mu \phi_{\mathcal{I}}(\mu)$ is concave on $[1, \infty)$, and therefore is Lipshitz on any interval $[r, t]$ with $r>1$. Since $a_{\mathcal{I}} / h_{\mathcal{I}}>0$, there exists a $C>0$, 
depending on $\left(a_{\mathcal{I}}, h_{\mathcal{I}}\right)$ only, such that $V_{x} \leq C x$ for $x$ small enough. Therefore (G.9) becomes

$$
f_{l_{A}, l_{B}}\left((h)_{x},(a)_{x}\right)-f_{l_{A}, l_{B}}((h),(a)) \geq g_{l_{B}}\left(l_{B}+c x, x\right)-\left(C+c \frac{\beta-\alpha}{2}\right) x
$$

for $x$ small enough. By the concavity of $g_{l_{B}}$, and since $g_{l_{B}}\left(l_{B}+c x, 0\right)=0$, we can write $g_{l_{B}}\left(l_{B}+c x, x\right) \geq x \partial_{2} g_{l_{B}}\left(l_{B}+c x, x\right)$ for $x>0$. By the definition of $g_{l_{B}}$, and with (4.4), we obtain that

$$
\partial_{2} g_{l_{B}}\left(l_{B}+c x, x\right)=\left(1+\frac{c x}{l_{B}}\right) \partial_{2} \kappa\left(1+\frac{c x}{l_{B}}, \frac{c x}{l_{B}}\right) .
$$

We now recall den Hollander and Whittington (2006), Lemma 2.1.1, which claims that $\kappa$ is defined on $\mathrm{DOM}=\{(a, b): a \geq 1+b, b \geq 0\}$ and is analytic on the interior of DOM. Moreover, in the proof of this lemma, an expression for $\partial_{b} \kappa(a, b)$ is provided that is valid on the interior of DOM. From this expression, and since $c>1$, we can check that $\lim _{s \downarrow 0} \partial_{2} \kappa(1+c s, s)=\infty$, which suffices to conclude that the right-hand side of (G.9) is strictly positive for $x$ small enough. This completes the proof of the existence in Lemma G.2.

Uniqueness. The uniqueness of $(\bar{h}),(\bar{a})$ is a straightforward consequence of the strict concavity of $g_{l_{A}}$ and $g_{l_{B}}$ when $l_{A} \neq 0$ and $l_{B} \neq 0$ and of the concavity of $g_{0}$ and $\widetilde{g}$. We will not write out the proof in detail, because it requires us to distinguish between the cases $\Theta \in \overline{\mathcal{V}}_{\text {int }, M}$ and $\Theta \in \overline{\mathcal{V}}_{\text {nint }, M}$, between $l_{k}=0$ and $l_{k} \neq 0, k \in\{A, B\}$, and also between $x_{\Theta}=1$ and $x_{\Theta}=2$. The latter distinctions are tedious, but no technical difficulties arise.

We resume the proof of Proposition G.1. We pick $u \in \mathcal{B}_{\overline{\mathcal{V}}_{M}}$, and for each $\Theta \in \overline{\mathcal{V}}_{M}$ we apply Lemma G.2 at $\Theta, u_{\Theta}$, to obtain a $(\bar{h})_{\Theta},(\bar{a})_{\Theta} \in \mathcal{L}\left(\Theta ; u_{\Theta}\right)$ satisfying (i-iv). We set $(\bar{h}): \Theta \in \overline{\mathcal{V}}_{M} \mapsto \bar{h}_{\Theta}$ and $(\bar{a}): \Theta \in \overline{\mathcal{V}}_{M} \mapsto \bar{a}_{\Theta}$, and we recall (4.60). If we can show that $\Theta \mapsto(\bar{h})_{\Theta}$ is Borel, then it follows that $(\bar{h}) \in \mathcal{E}$, because (ii) and the fact that $(\bar{h})_{\Theta},(\bar{a})_{\Theta} \in \mathcal{L}\left(\Theta ; u_{\Theta}\right)$ for $\Theta \in \overline{\mathcal{V}}_{M}$ ensure that the other conditions required to belong to $\mathcal{E}$ are fulfilled by $(\bar{h})$. Moreover, if we can we show that $\Theta \mapsto(\bar{a})_{\Theta}$ is Borel, then the proof of Proposition G.1 will be complete, because we can set

$$
\left(\bar{r}_{A}(\Theta), \bar{r}_{B}(\Theta), \bar{r}_{\mathcal{I}}(\Theta)\right)=\left(\frac{\bar{a}_{A}(\Theta)}{h_{A}(\Theta)}, \frac{\bar{a}_{B}(\Theta)}{h_{B}(\Theta)}, \frac{\bar{a}_{\mathcal{I}}(\Theta)}{h_{\mathcal{I}}(\Theta)}\right), \quad \Theta \in \overline{\mathcal{V}}_{M},
$$

with the convention $\bar{r}_{k}(\Theta)=1$ when $\bar{a}_{k}(\Theta)=\bar{h}_{k}(\Theta)=0$ for $k \in\{A, B, \mathcal{I}\}$, after which $(\bar{r}) \in \mathcal{U}(h)$ and $(\bar{h}),(\bar{r})$ satisfy (G.2) and (G.3).

To complete the proof it remains to show that $\Theta \mapsto(\bar{h})_{\Theta},(\bar{a})_{\Theta}$ is Borel. Recall the partition

$$
\overline{\mathcal{V}}_{M}=\overline{\mathcal{V}}_{\text {int }, M} \cup\left(\cup_{(x, k) \in\{1,2\} \times\{A, B\}} \overline{\mathcal{V}}_{\text {int }, k, x, M}\right),
$$

and partition these five subsets in the right-hand side of (G.13) into smaller subsets depending on the values taken by $l_{A}$ and $l_{B}$. For $\overline{\mathcal{V}}_{\text {int }, M}$, this gives

$$
\begin{aligned}
\overline{\mathcal{V}}_{\mathrm{int}, M}= & \left\{\Theta \in \overline{\mathcal{V}}_{\mathrm{int}, M}: l_{A}, l_{B}>0\right\} \cup\left\{\Theta \in \overline{\mathcal{V}}_{\mathrm{int}, M}: l_{A}>0, l_{B}=0\right\} \\
& \cup\left\{\Theta \in \overline{\mathcal{V}}_{\mathrm{int}, M}: l_{A}=0, l_{B}>0\right\} \cup\left\{\Theta \in \overline{\mathcal{V}}_{\mathrm{int}, M}: l_{A}=l_{B}=0\right\},
\end{aligned}
$$

and on each of these subsets the fact that $(\bar{h})_{\Theta},(\bar{a})_{\Theta}$ are the unique elements in $\mathcal{L}\left(\Theta ; u_{\Theta}\right)$ satisfying (i-iv) implies that $\Theta \mapsto(\bar{h})_{\Theta},(\bar{a})_{\Theta}$ are continuous and therefore Borel. Since each subsets in the right-hand side of (G.14) belongs to the Borel $\sigma$ field generated by $d_{M}$ (recall (C.7)), we can conclude that $\Theta \mapsto(\bar{h})_{\Theta},(\bar{a})_{\Theta}$ are Borel on $\overline{\mathcal{V}}_{M}$. This completes the proof of Proposition G.1. 


\section{References}

F. Caravenna, F. den Hollander and N. Pétrélis. Lectures on random polymers. In Probability and statistical physics in two and more dimensions, volume 15 of Clay Math. Proc., pages 319-393. Amer. Math. Soc., Providence, RI (2012). MR3025394.

G. Giacomin. Random Polymer Models. Imperial College Press, London (2007). ISBN 978-1-86094-786-5; 1-86094-786-7. MR2380992.

F. den Hollander. Large Deviations, volume 14 of Fields Institute Monographs. American Mathematical Society, Providence RI (2000). ISBN 0-8218-4435-0.

F. den Hollander. Random Polymers, volume 1974 of Lecture Notes in Mathematics. Springer-Verlag, Berlin (2009). ISBN 978-3-642-00332-5. Lectures from the 37th Probability Summer School held in Saint-Flour, 2007. MR2504175.

F. den Hollander and N. Pétrélis. On the localized phase of a copolymer in an emulsion: subcritical percolation regime. J. Stat. Phys. 134 (2), 209-241 (2009a). MR24857157.

F. den Hollander and N. Pétrélis. On the localized phase of a copolymer in an emulsion: supercritical percolation regime. Comm. Math. Phys. 285 (3), 825871 (2009b). MR2470907.

F. den Hollander and N. Pétrélis. A mathematical model for a copolymer in an emulsion. J. Math. Chem. 48 (1), 83-94 (2010). MR2657918.

F. den Hollander and N. Pétrélis. Free energy of a copolymer in a micro-emulsion. ArXiv Mathematics e-prints (2012). arXiv: 1204.1234.

F. den Hollander and S. G. Whittington. Localization transition for a copolymer in an emulsion. Teor. Veroyatn. Primen. 51 (1), 193-240 (2006). MR2324174.

F. Rassoul-Agha, T. Seppäläinen and A. Yilmaz. Quenched free energy and large deviations for random walks in random potentials. Comm. Pure Appl. Math. 66 (2), 202-244 (2013). MR2999296.

F. Rassoul-Agha, T. Seppäläinen and A. Yilmaz. Variational formulas and disorder regimes of random walks in random potentials (2016+). To appear in Bernoulli.

M. Talagrand. A new look at independence. Ann. Probab. 24 (1), 1-34 (1996). MR1387624. 